\title{
A Study of the Impact Response of Discrete Regions of the Human Cadaver Brain
}

\author{
by \\ Scott Dutrisac \\ A Thesis submitted to \\ the Faculty of Graduate Studies and Research \\ in partial fulfilment of \\ the requirements for the degree of \\ Master of Applied Science \\ Ottawa-Carleton Institute for \\ Mechanical and Aerospace Engineering \\ Department of Mechanical and Aerospace Engineering \\ Carleton University \\ Ottawa, Ontario, Canada \\ September 23, 2020 \\ Copyright $(9$ \\ 2020 - Scott Dutrisac
}


The undersigned recommend to the Faculty of Graduate Studies and Research acceptance of the Thesis

\title{
A Study of the Impact Response of Discrete Regions of the Human Cadaver Brain
}

\author{
Submitted by Scott Dutrisac \\ in partial fulfilment of the requirements for the degree of \\ Master of Applied Science
}

O. E. Petel, Thesis Co-Supervisor

H. Frei, Thesis Co-Supervisor

R. Miller, Department Chair

Carleton University

2020 


\section{Abstract}

The risk of trauma to the brain due to head impacts is high, despite widespread use of protective equipment and injury mitigation efforts. Mitigation techniques are developed through simulations that utilize complex finite element models of the brain and head. Validation of such models is limited, as existing empirical data is sparse. Due to technical constraints, empirical studies have only revealed broad brain tissue deformation. The objective of this study was to develop a comprehensive methodology for measuring the displacement of discrete brain structures during impact. An advanced X-ray system was used to capture brain motion for two cadaveric specimens at 7,500 fps. Displacement of brain structures was determined for 7 impacts on each specimen. Motion trends were region dependent, with some regions exhibiting multi-modal displacement. Displacement of discrete structures including the corpus callosum was measured. These methods will help clarify the response of the brain to impact. 


\section{Acknowledgments}

When I started working on this project in the fall of 2017, I was fairly certain that next two years would be a breeze. I am humbled to report that I was very wrong. The last three years, have been a roller-coaster of excitement, challenges, incredible learning - all of which were wonderful, but none of which were a breeze. Over this time, I have been involved with some exciting work, been to some thrilling places, and experienced the fallout of the 2020 Pandemic. Despite these ups and downs, I have still managed to reach a destination. While I take a moment to reflect on how I got here, I find it necessary to acknowledge the folks who helped make it happen.

I must first recognize my co-supervisors, Professors Oren Petel and Hanspeter Frei, who convinced me to join their team years ago. Each of these outstanding professors has boosted my abilities and this work would not be remotely possible without their contributions. I have truly enjoyed working as a colleague on this project and others that may come along in the future. To Oren, thank you for your knowledge, insights, patience, fair treatment, and willingness-to-stay-in-the-lab-until-the-sun-comes-up. These experiences have helped me become a better student, teacher, and researcher. To Hanspeter, if not for your expertise in cadaver work, encouragement, and advice, I would still be stumbling around confused about the difference between a scalpel and a box cutter.

This work was funded by the CIHR and NSERC through the Collaborative Health Research Program (CPG-151967). O.E.P. received funding support from Ontario Ministry of Research and Innovation - Early Research Award (ER-18-14-063). O.E.P. received funding for the cineradiography equipment from the Canada Foundation for Innovation (project 32933). 
This research was supported and guided with the invaluable assistance and clinical expertise of colleagues and partners in Human Kinetics at University of Ottawa, Professor T. Blaine Hoshizaki, and his team of PhDs, A. Post., C. Karton, D. Koncan., K. Taylor, among others. The staff and faculty in the Faculty of Medicine University of Ottawa, S. Goodwin, Dr. A. Jalali, and of course the unbelievably knowledgeable and helpful neurosurgeons and medical imaging experts, Dr. S. Brien, Dr. K. Poon, Dr. G. Cron, and Dr. G. Melkus. Your contributions to this work are greatly appreciated.

An immense amount of thanks must be directed at large group of people. Insightful conversations, design feedback, and even clicking-a-button-1500-times while debugging systems, do not go unnoticed, or without thanks to all of my lab and office mates, past and present. Thanks also to the entire support and technical staff in the Mechanical and Aerospace Department at Carleton University, whose guidance in administrative matters, assistance in manufacturing apparatus, and troubleshooting systems, was unmatched.

My friends and family must also be recognized here. They have listened, without complaining, to me yammer on for hours about brains and X-rays. Their guiding words, unexpected visits, weekend BBQs, have all been helpful on this journey. The advice, encouragement, and distractions ${ }^{\text {HelloTaskMaster }}$ were much needed when the going got tough. In no particular order, André, Linda, Valerie, Mark, James, Martina, John, Celia, Tim, Matt, Andrew, Adrian, Stefano, Ron, Nick, Jacob, Zac, Nathalie - and many more - you have my gratitude.

While the contributions of those above were unbelievably necessary and appreciated, this work would not have been remotely possible, without the support of my fianceé, Monica. Her patience, encouragement, round-the-clock snack supply, and programming advice (she's a software engineer, after all), are the greatest gifts anyone could offer. I can't thank her enough and I am excited to spend the rest of my life with her.

I'm not sure what's next, but as Red Green would say, "Till next time, keep yer stick on the ice". 


\section{Preface}

The work presented in this document was performed at the Carleton Univer-

sity Impact Research Laboratory with collaboration from the Neurotrauma Impact Science Laboratory at University of Ottawa. Cadaver specimens were obtained through the University of Ottawa Body Bequeathal Program.

\section{Ethics Approval}

This work was approved by the Carleton University Research Ethics Board, REB\#107190.

\section{Contributions}

Image processing, video stabilization, particle tracking, and displacement measurement methods were developed in MATLAB by Scott Dutrisac and performed by him and Jennifer Rovt. The marker design experiment was conceived and performed by Scott Dutrisac with laboratory assistance from MacKenzie Brannen. The high-speed X-ray imaging system was conceived and implemented by Professor Oren Petel with assistance and component development from Stephane Magnan, Scott Dutrisac, Sheng Xu, Ashley Mazurkiewicz, and MacKenzie Brannen. The PMHS impact methodology and apparatus were developed by Scott Dutrisac with guidance from Professor Oren Petel, Professor Hanspeter Frei, Dr. Susan Brein, Dr. Katherine Poon, Professor Blaine Hoshizaki, Dr. Andrew Post, Dr. David Koncan, Dr. Karen Taylor, and Dr. Clara Karton. The PMHS protocol was performed by Scott Dutrisac with laboratory assistance from Professors Petel and Frei, Dr. 
Andrew Post, Jennifer Rovt, Mary-Jo Weir Weiss, MacKenzie Brannen, and Klara Doelle. Specimens were prepared at University of Ottawa by Prosector Shannon Goodwin. MRI imaging was performed at The Royal Ottawa Hospital by Dr. Greg Cron and Dr. Gerd Melkus.

\section{Use of Images}

Images not created by the author have been obtained and used per "Fair Dealing" under the Canadian Copyright Act, R.S.C., 1985, c. C-42 [1] and "Fair Use" under Section 107 of the U.S. Copyright Act [2]. These images are used explicitly for the purpose of scholarship, private study, and research. Where applicable, additional licensing has been requested from the Copyright Clearance Centre through RightsLink. Original creators and publications have been credited and cited. 


\section{Table of Contents}

Abstract iii

Acknowledgments iv

Preface vi vi vis

Table of Contents viii

List of Tables $\quad$ xii

List of Figures $\quad$ xiii

Nomenclature $\quad$ xix

\begin{tabular}{lll}
\hline 1 & Introduction & 1
\end{tabular}

1.1 Traumatic Brain Injury . . . . . . . . . . . . . . . . . . . 1

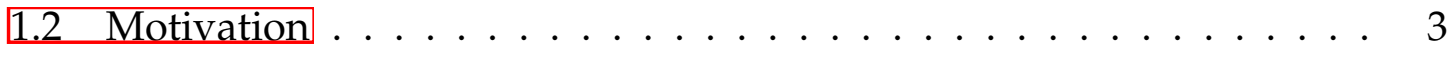

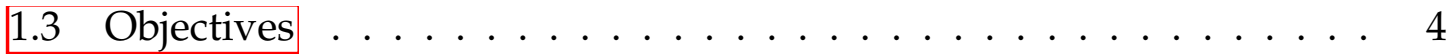

1.4 Thesis Overview $\ldots \ldots \ldots \ldots \ldots$

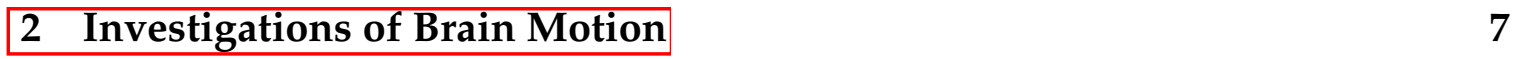

2.1 Methodological Developments . . . . . . . . . . . . . 8

2.1 .1 Kinematic Data Collection . . . . . . . . . . . . . . 9

2.1 .2 Pressure Measurement . . . . . . . . . . . . . . . . . 13 
2.1 .3 Observing Brain Motion . . . . . . . . . . . . . . . . . 14

2.1 .4 PMHSPreparation . . . . . . . . . . . . . . . . . . . . . 19

2.1 .5 Alternative Techniques . . . . . . . . . . . . . . . . 23

2.2 Overview of Key Findings . . . . . . . . . . . . . . . . . . . . 24

2.3 Summary . . . . . . . . . . . . . . . . . 26

\begin{tabular}{|lll}
\hline 3 & Image Capture and Processing & 28
\end{tabular}

$3.1 \quad$ High Speed Cineradiography at Carleton . . . . . . . . . . . . . . . . 29

3.1 .1 Use in Marker Development . . . . . . . . . . . . . . . . . . . 29

3.1 .2 Use in PMHS Study . . . . . . . . . . . . . . . . . . 33

3.2 Data Extraction . . . . . . . . . . . . . . . . . . . 34

$3.2 .1 \quad$ Clarity Improvements $\ldots \ldots \ldots$

3.2 .2 Stabilizaiton . . . . . . . . . . . . . . . . . . . . 39

3.2.3 Determining Target Displacement . . . . . . . . . . . . 43

3.2 .4 Centre of Mass . . . . . . . . . . . . . . . 46

\begin{tabular}{|lll}
4 & Contrast Marker Development & 49
\end{tabular}

$4.1 \quad$ Experimental Details . . . . . . . . . . . . . . . . . . 50

$4.1 .1 \quad$ Marker Preparation . . . . . . . . . . . . . . . . . . 52

$4.1 .2 \quad$ Drop Testing. . . . . . . . . . . . . . . . . . . . . . 55

4.1 .3 Data Collection . . . . . . . . . . . . . . . . . . . 55

4.1 .4 Analysis $\ldots \ldots \ldots \ldots \ldots$

4.2 Results . . . . . . . . . . . . . . . . . . . . . . 65

$4.2 .1 \quad$ New Marker Designs. . . . . . . . . . . . . . . . . . . . 65

$4.2 .2 \quad$ NDT, Lead Markers … . . . . . . . . . . . . . . . . . 69

4.2 .3 Discussion . . . . . . . . . . . . . . . . . . . 71

4.2 .4 Summary $\ldots \ldots \ldots \ldots \ldots \ldots \ldots \ldots$ 
5 PMHS Experiment Details 75

$5.1 \quad$ PMHS Preparation . . . . . . . . . . . . . . 76

5.2 Diagnostic Imaging and Evaluation. . . . . . . . . . . . 77

5.3 Specimen Mounting and Instrumentation $\ldots \ldots \ldots \ldots$

5.3 .1 Mounting . . . . . . . . . . . . . . . . 80

5.3 .2 Instrumentation . . . . . . . . . . . . . . . . . 84

5.3 .3 Contrast Marker Insertion . . . . . . . . . . . . . . . . 86

5.3 .4 Artificial Perfusion . . . . . . . . . . . . . . . . . . . 94

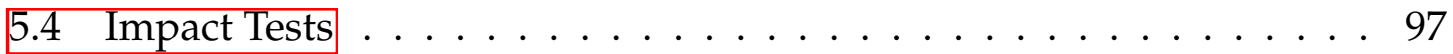

$5.4 .1 \quad$ Preliminary Imaging $\ldots \ldots \ldots$

5.4 .2 Kinematics and Data Logging. . . . . . . . . . . . . . . 100

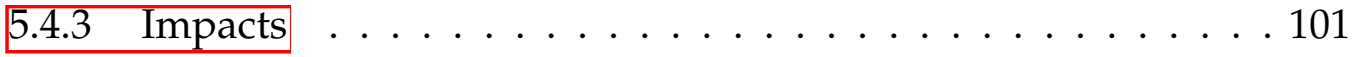

5.5 Post-Experiment Procedures . . . . . . . . . . . . . . . . 102

6 PMHS Results and Discussion 104

6.1 PMHS Impact Results . . . . . . . . . . . . . . . . . . . . . . . 104

6.1 .1 Kinematic Response . . . . . . . . . . . . . . . . . . 105

6.1 .2 Pressure Response . . . . . . . . . . . . . . . . . . . . . . 109

6.1 .3 Brain Tissue Displacement. . . . . . . . . . . . . . . . . . . . 111

6.2 Discussion of PMHS Datat . . . . . . . . . . . . . . . . . 114

6.2 .1 Study Limitations . . . . . . . . . . . . . . . . . . . . . . . 114

6.2 .2 Validation of Data $\ldots \ldots \ldots \ldots \ldots$

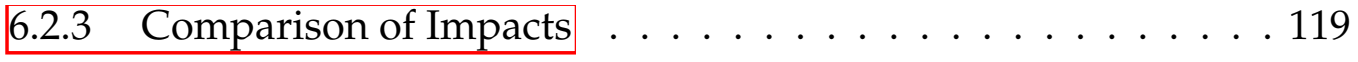

6.2 .4 Regional Displacements in the PMHS Brain. . . . . . . . . 127

\begin{tabular}{lll}
\hline 7 Conclusion & 140
\end{tabular}

\begin{tabular}{ll}
\hline List of References & 145
\end{tabular} 
Appendix A Artificial Cerebrospinal Fluid Formula

Appendix B Apparatus Drawings

152

\begin{tabular}{lll}
\hline Appendix C Impact Protocol & 158
\end{tabular}

\begin{tabular}{lll}
\hline Appendix D Additional Tables and Plots & 160
\end{tabular}

\begin{tabular}{|lll}
\hline Appendix E Excluded Markers & 204
\end{tabular}

\begin{tabular}{|lll}
\hline Appendix F & Additional Imaging and MRI & 206
\end{tabular} 


\section{List of Tables}

1 Taguchi L-9 array of marker designs $\ldots \ldots \ldots \ldots$

2 Experimental outcomes from drop test and respective $S N r$. . . . . 66

$3 \quad$ Mean SNrat Peak 1. . . . . . . . . . . . . . . . . . . . . 68

$4 \quad$ Mean $S N r$ at Peak 2 . . . . . . . . . . . . . . . . 68

5 Parameter sensitivity (\%)from ANOVA $\ldots \ldots \ldots$

$6 \quad$ Experimental outcomes from drop test and respective $S N r . \ldots \ldots 71$

$7 \quad$ Impactor cap stiffness . . . . . . . . . . . . . . . . . . . . . . . . 101

8 Summary of impacts for PMHS specimens . . . . . . . . . . . 105

9 Peak resultant linear and angular acceleration . . . . . . . . . 106

10 Adjusted peak coup pressure - Specimen 2871 . . . . . . . . . . . . 109

11 Peak marker displacement . . . . . . . . . . . . . . . . . . . . . 112

12 Marker count per region-Specimen $2871 \ldots \ldots \ldots$

13 Planned impact protocol for PMHS specimens . . . . . . . . . . . . 159

14 Specimen physical and anthropometric data $\ldots \ldots \ldots$. . . . . 160

15 Offset distances for accelerometer to CG . . . . . . . . . . . . . . . 161

16 Millimetre-pixel scales . . . . . . . . . . . . . . . . . . . . 166

17 Marker exclusions-Specimen $2842 \ldots \ldots$. . . . . . . . . . 205

18 Marker exclusions - Specimen 2871 . . . . . . . . . . . . . . . . 205

19 Location of markers per MRI review -Specimen 2871 . . . . . . . . 206 


\section{List of Figures}

1 Typical quantitative measurements . . . . . . . . . . . . . . 9

2 Wayne State Tolerance Curve . . . . . . . . . . . . . . . 10

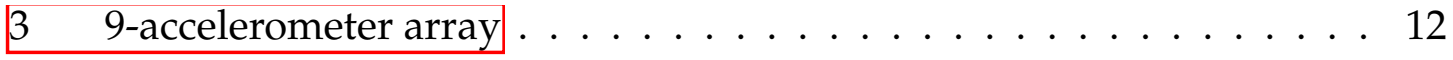

$4 \quad$ Flash X-ray techniques . . . . . . . . . . . . . . . . . . . 16

5 High speed bi-plane X-ray system . . . . . . . . . . . . 17

$6 \quad$ NDT positions . . . . . . . . . . . . . . . . . . . . . . . . . . 19

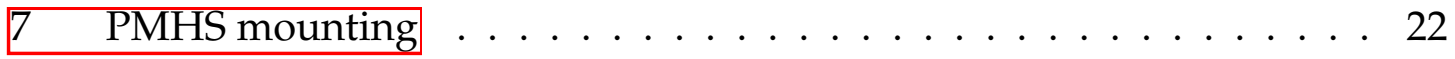

8 Overview of HSXR system . . . . . . . . . . . . . . . . . 30

$9 \quad$ HSXR use in marker study . . . . . . . . . . . . . . . . . . . . 32

10 HSXR use in PMHS study . . . . . . . . . . . . . . . . . . . . 34

11 Contrast adjustment example . . . . . . . . . . . . . . . . 37

12 Flat field correction example . . . . . . . . . . . . . . . . . 38

13 Stabilization process $\ldots \ldots \ldots \ldots \ldots \ldots$. . . . . . . . . 41

14 Stabilization . . . . . . . . . . . . . . . . . . 42

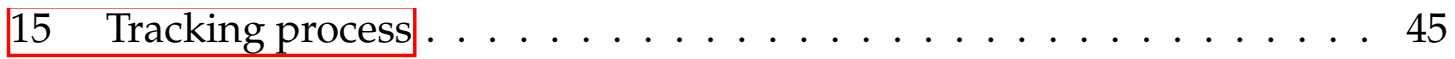

$16 \quad$ CG estimation example . . . . . . . . . . . . . . . . . . . 48

17 Marker test block schematic . . . . . . . . . . . . . . . . . 51

18 NDT orientations $\ldots \ldots \ldots \ldots \ldots \ldots \ldots \ldots$

19 Drop tower schematic . . . . . . . . . . . . . . . . 56

$20 \quad$ Marker test block photo $\ldots \ldots \ldots \ldots \ldots$ 
21 Test blocks and corresponding radiographs . . . . . . . . . . . . 59

22 Determining moment of impact . . . . . . . . . . . . . . . 60

23 Mean response and confidence interval . . . . . . . . . . . 62

24 Vertical displacement of new markers … . . . . . . . . . 67

25 Vertical displacement of existing markers $\ldots \ldots \ldots \ldots . \ldots 70$

26 Response due to NDT orientation . . . . . . . . . . . . . . . 73

$27 \quad$ PMHS illustration . . . . . . . . . . . . . . . . . . 77

28 Sample of tomographic image . . . . . . . . . . . . . . . . . . . 79

29 Target positions for markers $\ldots \ldots \ldots$. . . . . . . . . . . . 79

30 Anthropometric measurements . . . . . . . . . . . . . 80

31 Neck collar designs . . . . . . . . . . . . . . . . . . . . . . . . . . 82

32 Neck collar alignment . . . . . . . . . . . . . . . . . . . . 82

33 PMHS with collar mounted $\ldots \ldots \ldots \ldots$. . . . . . . 83

34 Scalp access points . . . . . . . . . . . . . . . 85

35 Positoion of access holes in skull $\ldots \ldots \ldots \ldots$. . . . . . 85

36 Hole drilling in PMHS specimen $\ldots \ldots \ldots \ldots$. . . . . . 88

37 Marker insertion tool . . . . . . . . . . . . . . . . . . . . . . . 89

38 Contrast marker sheet $\ldots \ldots \ldots \ldots$. . . . . . . . . . 90

39 Marker insertion process $\ldots \ldots \ldots$. . . . . . . . . . . . 91

$40 \quad$ Marker number index -Specimen $2842 \ldots \ldots \ldots$

$41 \quad$ Marker number index-Specimen $2871 \ldots \ldots$. . . . . . . . . 93

42 Perfusion system $\ldots \ldots \ldots \ldots$

$43 \quad \operatorname{Impact}$ system $\ldots \ldots \ldots \ldots \ldots$. . . . . . . . . . . 97

44 High resolution projection of instrumented PMHS . . . . . . . . . . 99

45 Post-experiment MRI . . . . . . . . . . . . . . . . . . . . . 103

46 Linear and angular acceleration curves . . . . . . . . . . . . . . 107

$47 \quad$ X-ray images corresponding to kinematics. . . . . . . . . . . . 108 
48 Coup pressure $-2871-6 \ldots \ldots \ldots$. . . . . . . . . . . . . 110

49 Motion paths - Specimen $2871 \ldots \ldots$. . . . . . . . . . . . . 113

50 Pressure compared to previous studies . . . . . . . . . . . . 118

51 Displacement paths - 2871-3 . . . . . . . . . . . . . . . . 120

52 Displacement paths - 2842-7 . . . . . . . . . . . . . . . . . . . 121

53 Displacement paths - 2871-1 . . . . . . . . . . . . . . . . 122

54 Displacement paths - 2871-5 . . . . . . . . . . . . . . . . . 122

55 Displacement paths - 2842-4 . . . . . . . . . . . . . . . . . . 123

56 Displacement paths - 2871-3 . . . . . . . . . . . . . . . . . . 124

57 Displacement paths - 2871-6 . . . . . . . . . . . . . . . . 125

58 Displacement paths - 2871-7 . . . . . . . . . . . . . . . . . 126

59 Parietal, frontal, occipital, cerebellar displacements - 2871-1 . . . . . . 130

60 Corpus callosum, basal ganglia, thalamus, cingulate gyrus displace-

ments - 2871-1 . . . . . . . . . . . . . . . . . . . . . 131

61 Parietal, frontal, occipital, cerebellar displacements - 2871-3 . . . . . 132

62 Corpus callosum, basal ganglia, thalamus, cingulate gyrus displacements - 2871-3 . . . . . . . . . . . . . . . . . . . 133

63 Superior and inferior frontal displacements - 2871-3 . . . . . . . . . 135

64 Occipital and cerebellar displacements 2871-6, 2871-7 . . . . . . . . 136

65 Thalamus and basal ganglia displacements 2871-6, 2871-7 . . . . . . 137

66 Coordinate System for Accelerometer Offset . . . . . . . . . . . . . 161

67 Acceleration compared to previous studies . . . . . . . . . . . 162

68 Coup pressure $-2871-1 \ldots \ldots \ldots \ldots$. . . . . . . . . . . . . . . . .

69 Coup pressure $-2871-2 \ldots \ldots \ldots \ldots$. . . . . . . . . . . . . . . .

70 Coup pressure - 2871-3 . . . . . . . . . . . . . . . . . . . . . 164

71 Coup pressure - 2871-4 . . . . . . . . . . . . . . . . . . . . 164

72 Coup pressure $-2871-5 \ldots \ldots \ldots \ldots$. . . . . . . . . 165 
73 Coup pressure - 2871-7 . . . . . . . . . . . . . . . . . . 165

74 Displacement paths - 2842-2 . . . . . . . . . . . . . . . . . 167

75 Displacement paths - 2871-2 . . . . . . . . . . . . . . . . 167

76 Displacement paths - 2871-4 . . . . . . . . . . . . . . . . 168

77 Displacement of basal ganglia - 2871-1 . . . . . . . . . . . . . . 169

78 Displacement of brainstem - 2871-1 . . . . . . . . . . . . . . . . 169

79 Displacement of cerebellum - 2871-1 . . . . . . . . . . . . . 170

80 Displacement of cingulate gyrus - 2871-1 . . . . . . . . . . . . 170

81 Displacement of corpus callosum - 2871-1 . . . . . . . . . . . . 171

82 Displacement of frontal lobe - 2871-1 . . . . . . . . . . . . . 171

83 Displacement of occipital lobe - 2871-1 . . . . . . . . . . . . . . . 172

84 Displacement of parietal lobe - 2871-1 . . . . . . . . . . . . . . . 172

85 Displacement of temporal lobe - 2871-1 . . . . . . . . . . . . 173

86 Displacement of thalamus - 2871-1 . . . . . . . . . . . . . 173

87 Displacement of basal ganglia - 2871-2 . . . . . . . . . . . . . 174

88 Displacement of brainstem - 2871-2 . . . . . . . . . . . . . . . . . . 174

89 Displacement of cerebellum - 2871-2 . . . . . . . . . . . . . . 175

90 Displacement of cingulate gyrus - 2871-2 . . . . . . . . . . . . 175

91 Displacement of corpus callosum - 2871-2 . . . . . . . . . . . 176

92 Displacement of frontal lobe - 2871-2 . . . . . . . . . . . . . 176

93 Displacement of occipital lobe - 2871-2 . . . . . . . . . . . . . 177

94 Displacement of parietal lobe - 2871-2 . . . . . . . . . . . . . . 177

95 Displacement of temporal lobe - 2871-2 $\ldots \ldots \ldots \ldots$

96 Displacement of thalamus - 2871-2 . . . . . . . . . . . . 178

97 Displacement of basal ganglia - 2871-3 . . . . . . . . . . . . . . . 179

98 Displacement of brainstem - 2871-3 . . . . . . . . . . . . . . . 179

99 Displacement of cerebellum - 2871-3 . . . . . . . . . . . . . . . 180 
100 Displacement of cingulate gyrus - 2871-3 … . . . . . . . 180

101 Displacement of corpus callosum - 2871-3 . . . . . . . . . . . . . 181

102 Displacement of frontal lobe - 2871-3 . . . . . . . . . . . . . . . 181

103 Displacement of occipital lobe - 2871-3 . . . . . . . . . . . . . 182

104 Displacement of parietal lobe - 2871-3 . . . . . . . . . . . . . 182

105 Displacement of temporal lobe - 2871-3 . . . . . . . . . . . . 183

106 Displacement of thalamus $-2871-3 \ldots \ldots \ldots$. . . . . . . 183

107 Displacement of basal ganglia - 2871-4 . . . . . . . . . . . . . . 184

108 Displacement of brainstem - 2871-4 . . . . . . . . . . . . . . . 184

109 Displacement of cerebellum - 2871-4 . . . . . . . . . . . . . . . 185

110 Displacement of cingulate gyrus - 2871-4 . . . . . . . . . . . . . 185

111 Displacement of corpus callosum - 2871-4 . . . . . . . . . . . . . . 186

112 Displacement of frontal lobe - 2871-4 . . . . . . . . . . . . . 186

113 Displacement of occipital lobe - 2871-4 . . . . . . . . . . . . 187

114 Displacement of parietal lobe - 2871-4 … . . . . . . . . . . 187

115 Displacement of temporal lobe - 2871-4 . . . . . . . . . . . . . 188

116 Displacement of thalamus - 2871-4 . . . . . . . . . . . . . 188

117 Displacement of basal ganglia - 2871-5 . . . . . . . . . . . . . . 189

118 Displacement of brainstem - 2871-5 . . . . . . . . . . . . . . . . 189

119 Displacement of cerebellum - 2871-5 … . . . . . . . . . . . . 190

120 Displacement of cingulate gyrus - 2871-5 . . . . . . . . . . . . 190

121 Displacement of corpus callosum - 2871-5 . . . . . . . . . . . . . . 191

122 Displacement of frontal lobe - 2871-5 . . . . . . . . . . . . . . 191

123 Displacement of occipital lobe - 2871-5 . . . . . . . . . . . . . 192

124 Displacement of parietal lobe - 2871-5 . . . . . . . . . . . . . . 192

125 Displacement of temporal lobe - 2871-5 . . . . . . . . . . . . 193

126 Displacement of thalamus - 2871-5 . . . . . . . . . . . . . . 193 
127 Displacement of basal ganglia - 2871-6 . . . . . . . . . . . . . . 194

128 Displacement of brainstem - 2871-6 . . . . . . . . . . . . . . . . 194

129 Displacement of cerebellum - 2871-6 . . . . . . . . . . . . . . 195

130 Displacement of cingulate gyrus $-2871-6 \ldots \ldots$. . . . . . . . 195

131 Displacement of corpus callosum - 2871-6 . . . . . . . . . . . . . 196

132 Displacement of frontal lobe - 2871-6 . . . . . . . . . . . . . 196

133 Displacement of occipital lobe - 2871-6 . . . . . . . . . . . . . 197

134 Displacement of parietal lobe - 2871-6 . . . . . . . . . . . . . 197

135 Displacement of temporal lobe - 2871-6 . . . . . . . . . . . . 198

136 Displacement of thalamus $-2871-6 \ldots \ldots$. . . . . . . . . . 198

137 Displacement of basal ganglia - 2871-7 . . . . . . . . . . . . . . . 199

138 Displacement of brainstem - 2871-7 . . . . . . . . . . . . . . . 199

139 Displacement of cerebellum - 2871-7 . . . . . . . . . . . . . 200

140 Displacement of cingulate gyrus - 2871-7 . . . . . . . . . . 200

141 Displacement of corpus callosum - 2871-7 . . . . . . . . . . . . . . 201

142 Displacement of frontal lobe - 2871-7 . . . . . . . . . . . . . . 201

143 Displacement of occipital lobe - 2871-7 . . . . . . . . . . . . . 202

144 Displacement of parietal lobe - 2871-7 . . . . . . . . . . . . . . . 202

145 Displacement of temporal lobe - 2871-7 . . . . . . . . . . . . 203

146 Displacement of thalamus - 2871-7 . . . . . . . . . . . . . . . 203

147 MRI-Specimen $2842 \ldots \ldots$. . . . . . . . . . . . . . . . . . . . . . 210

148 MRI-Specimen 2871 . . . . . . . . . . . . . . . . . . . . . . . . . 210

149 Corpus callosum MRI - Specimen 2871 . . . . . . . . . . . . . 211

150 Brainstem MRI - Specimen 2871 . . . . . . . . . . . . . . . . 211 


\section{Nomenclature}

\section{Latin and Greek Characters}

\begin{tabular}{|c|c|c|}
\hline Symbol & Description & Units \\
\hline$a$ & Linear acceleration & {$\left[m / s^{2}\right]$} \\
\hline$E$ & Modulus of elasticity & {$[G P a]$} \\
\hline G & Sum of results & {$[--]$} \\
\hline$g$ & Linear acceleration & {$\left[9.81 \mathrm{~m} / \mathrm{s}^{2}\right]$} \\
\hline$I$ & Pixel intensity & {$[--]$} \\
\hline$k$ & Count of PL combinations & {$[--]$} \\
\hline$L$ & Parameter level & {$[--]$} \\
\hline M & Number of vertical pixels & {$[--]$} \\
\hline$m_{j}$ & Linear sum of pixels for jth column & {$[--]$} \\
\hline$N$ & Number of horizontal pixels & {$[--]$} \\
\hline$n$ & Number of repeated drop trials & {$[--]$} \\
\hline$P$ & Parameter index & {$[--]$} \\
\hline
\end{tabular}




\begin{tabular}{|c|c|c|}
\hline$r_{A} / B$ & Offset from accelerometer to CG of head & {$[\mathrm{mm}]$} \\
\hline$S_{x}$ & Horizontal scaling factor & {$[--]$} \\
\hline$S_{y}$ & Vertical scaling factor & {$[--]$} \\
\hline SNr & Signal to noise ratio & {$[--]$} \\
\hline$S S_{t}$ & Total variance & {$[--]$} \\
\hline$S s_{e}$ & External noise variance & {$[--]$} \\
\hline$S s_{i}$ & Design parameter variance & {$[--]$} \\
\hline$T$ & Pixel-intensity moment & {$[--]$} \\
\hline$t$ & Time & {$[m s]$} \\
\hline$t_{x}$ & Horizontal translation & {$[p x]$} \\
\hline$t_{y}$ & Vertical translation & {$[p x]$} \\
\hline W & Pixel-intensity sum & {$[--$} \\
\hline$x$ & First frame horizontal coordinate & {$[p x]$} \\
\hline$x^{\prime}$ & Instantaneous frame horizontal coordinate & {$[p x]$} \\
\hline $\bar{x}$ & Horizontal component of CG & {$[p x]$} \\
\hline$y$ & First frame vertical component & {$[p x]$} \\
\hline$y^{\prime}$ & Instantaneous frame vertical component & {$[p x]$} \\
\hline
\end{tabular}




\section{Greek Characters}

\begin{tabular}{lll} 
Symbol & Description & Units \\
\hline$\alpha$ & Angular acceleration & {$\left[\mathrm{rad} / \mathrm{s}^{2}\right]$} \\
$\theta$ & Rotation angle & {$[\mathrm{rad}]$} \\
$\mu / \rho$ & Mass attenuation coefficient & {$\left[\mathrm{cm}^{2} / \mathrm{g}\right]$} \\
$\phi$ & Normalized output & {$[--]$} \\
$\chi_{n}$ & Neat material output metric & {$[--]$} \\
& & \\
$\chi_{t}$ & Test material output metric & {$[--]$} \\
& &
\end{tabular}

\section{List of Abbreviations}

\begin{tabular}{ll} 
Abbreviation & Description \\
\hline ANOVA & Analysis of variance \\
BaSO4 & Barium sulfate \\
CI & Centre of gravity \\
CT & Confidence interval \\
DAI & Diffuse axonal injury \\
DICOM & Digital Imaging and Communications in Medicine (file format)
\end{tabular}


FE

Finite element

FEM Finite element model

FPS Frames per second

HIC Head injury criterion

HSXR High-speed X-ray

MP Megapixel

MRI Magnetic resonance imaging

mTBI Mild traumatic brain injury

NDT Neutral density target

NHTSA National Highway Traffic Safety Association

NPT National Pipe Thread

PMHS Post-mortem human surrogate

ROI Region of interest

TBI Traumatic brain injury

TIFF Tagged image file format

WSTC Wayne State tolerance curve 


\section{Chapter 1}

\section{Introduction}

\subsection{Traumatic Brain Injury}

Traumatic brain injuries (TBIs) caused by impacts to the head are an ongoing serious health concern that can cause devastating long-term effects. These injuries can present a range of conditions from concussion (mild TBI), to severe neurological disorders, and death. Despite the use of protective equipment and risk minimization practices, incidence of these injuries continues to rise. A recent study in Canada identified that TBIs more than doubled from 2005 to 2014 [3], based on data collected from the Canadian Community Health Survey. In 2014, it is estimated that there were 155,000 TBIs in Canada, with most injuries occurring due to sports-related falls. In the United States, TBIs, including mTBI, made up more than 2.8 million visits to emergency departments, hospitalizations, and deaths in 2014 [4].

Depending on the impact conditions, TBIs can have various presentations, with two main characterizations. Injuries that affect focused regions of the brain, such as contusions, haematomas, haemorrhaging, and tearing of tissue in, or around the brain are classified as focal injuries. These injuries can be part of more complex injuries including penetrative head wounds [5]. Injuries related to acceleration 
and rotation of the head and brain are referred to as diffuse injuries, as they cause damage across the entire parenchyma of the brain. Although focal and diffuse injuries are characteristically different, a single traumatic event can cause both, resulting in complex damage to the brain. Typical diffuse injuries include intracranial swelling, ischaemia, and diffuse axonal injury (DAI). Axonal injuries present a broad range of neurological dysfunction, from headache and dizziness in mild cases, to coma in the most severe cases [6]. More severe DAIs result in focal lesions through the white matter of the brain, which can be observed using magnetic resonance imaging (MRI). The white matter damage is caused by the deformation of the brain following impact loading, which may result in shear and tensile failure of axons [6]. Although axonal damage can be widespread through the entire brain, it is frequently observed in the the corpus callosum and brainstem [6, 7]. While milder cases are also the result of damaged white matter, the damage is at a microscopic level, undetectable by clinical imaging systems [8] in living patients. As such, mild cases are diagnosed symptomatically, and are typically consistent with concussion symptoms [6].

Although there is some clinical understanding of effects of TBI and mTBI, thorough understanding of the mechanism of injury remains unclear. Treatment of these injuries is challenging, and associated with long recovery times. Mild injuries are served only by therapeutic treatments, with a broad range of effectiveness, varying from patient to patient [9]. Further investigation of the response of the brain to impact is needed to develop a better understanding of the associated damage. As these injuries present a substantive detriment to neurological health, their mitigation is of significance. 


\subsection{Motivation}

Broadly, the mitigation of traumatic brain injuries is built upon risk management and the use of protective equipment. Understanding the risks associated with impacts to the head allow injurious situations to be avoided if possible. Injury risk thresholds, like the Head Injury Criterion (HIC) [10], inform limits on acceptable and unacceptable risks, based on external impact characteristics like acceleration. These thresholds have been used to evaluate dangerous impact scenarios, such as vehicle collisions and sports-based impacts. Protective equipment is often designed to reduce impact severity, such that it falls within an acceptable range of injury risk. In particular, helmet testing methods have long used risk thresholds to evaluate their effectiveness in mitigating injury [11]. While these measures have resulted in an overall reduction in focal injuries seen in sports-related impacts, their effectiveness in reducing diffuse mTBI remains unseen [12].

The development of more rigorous injury risk assessments and advanced protective equipment may further reduce the severity of injury. Presently, numerical and computer modelling of the brain is used as means of evaluating helmets. A vast number of different models, each with its own specific fine-tuned properties, have been developed [13-15]. Advanced models have aimed to provide very high resolution, incorporating small structures like the often-damaged corpus callosum [15]. The simulations computed by the models are based on the kinematic properties of different impact scenarios. These kinematic properties are often developed from testing standards [11] or lab-based impacts using synthetic head forms [16, 17]. Simulations typically provide the response of the brain in terms of pressure, displacement, and strain. In comparing the model outputs across the different impact characteristics associated with helmet use, the efficacy of helmets is evaluated [17]. While these techniques have been widely used for evaluating 
the response of the brain to impact, limited validation of models has been possible. Testing helmets on living subjects is not feasible. Instead, pressure, displacement, and strain data captured from impacts performed on animal models and post-mortem human surrogates (PMHS) has been used for validation.

The animal and PMHS impact data presently used for validation is limited in biofidelity, precision, and quantity [18]. A number of studies have identified the bulk motion of the brain during lab-simulated injurious impact events, yet few have reported the deformation of discrete structures in the brain. In order to develop rigorous risk mitigation criteria and to create the next generation of protective equipment, a deeper understanding of the impact response of the brain is needed. To properly validate complex models presently used for simulations, as well as advanced future models, it is necessary to understand how each structure of the brain responds to impact with a high level of precision. Recent advances in imaging technology and insights in the improvement biofidelity in PMHS models will allow this data to be collected. Collection of this data will also support improved clinical understanding of the complexities of TBI.

\subsection{Objectives}

The work outlined in this thesis aims to identify and validate new tools and methods capable of observing and measuring the motion in the brain, both as a whole, and within anatomical regions of interest. The development of a protocol that permits the observation of brain motion is a complex task, incorporating elements from numerous fields of study. Image processing, apparatus design and optimisation, and experimental design have all played a part in the development and presentation of the results herein. With this in mind, this thesis was based on the following goals: 
1. To identify the methods by which PMHS brain motion can be measured and how results from future studies can be validated.

2. To develop tools, processes, and displacement measurement techniques for high-speed X-ray (HSXR) imaging of the PMHS brain.

3. To develop an experimental method for the preparation, impact-testing, and collection of relevant impact-response data of PMHS heads.

4. To validate the collected PMHS impact response data and evaluate it for use in identifying displacement of targeted anatomical structures in the brain.

5. To provide recommendations on methodology improvements for future HSXR and PMHS impact experiments.

\subsection{Thesis Overview}

This thesis is organized into seven chapters, covering background information, development of technical methods, validation and analysis of methods, and a presentation of preliminary results. A description of each chapter is provided below.

Chapter 1 Introduction - A brief overview of TBI and the state of injury mitigation measures, outline of experimental objectives and thesis content.

Chapter 2 Investigations of Brain Motion - A review of earlier studies on the observation of brain motion due to impact, with a focus on data acquisition methods, and key factors for experimental validation.

Chapter 3 Image Capture and Processing - Methods of image capture and processing used for marker development and PMHS studies.

Chapter 4 Contrast Marker Development - Development of an embedded tissue contrast marker with low mechanical interference. Taguchi methodology used to identify sensitive design parameters. 
Chapter 5 PMHS Experiment Details - Methodology for PMHS impact experiments, including instrumentation, imaging, and impacting.

Chapter 6: PMHS Results and Discussion - Validation of PMHS results against previously reported values, evaluation of methods for future use. Comparison of tissue displacements across specimens and impact intensities.

Chapter 7Concluding Remarks - Final summary of findings and recommendations for future PMHS work. 


\section{Chapter 2}

\section{Investigations of Brain Motion}

In order to further understand the damaging effects of head impacts, numerous studies have been published to identify and evaluate the causes and mechanics of traumatic brain injuries. Early investigations observed the response of animal brains and heads to external impact [19], focusing primarily on focal injuries due to technological limitations, restricting investigation to the brain surface. These early studies recognized that deeper trauma may be present following impacts to the head, given the large motions observed at the cortical surface of the brain. As the popularity of personal motor vehicles increased through the late 1940s [20], safety concerns spurred investigation of vehicle collisions and subsequent injury outcomes [21]. As understanding of impact injuries has improved with collision investigations, experiments involving more complex impact configurations have been developed [22-25]. Presently, the majority of mild traumatic brain injuries is associated with falls, while interest in sports-related injuries continues to rise, due to the prevalence of such injuries [3, 8]. These impacts represent their own unique challenges and mild traumatic brain injury risks due to the speeds, masses, orientations, and surfaces involved [26]. In order to better understand and mitigate the outcomes associated with impacts to the head, injury risk criteria, protective equipment, and clinical analyses have developed from extensive multidisciplinary 
impact studies. Over the course of several decades, studies have continued to improve upon past methods, leading to innovative ways to evaluate the response of the head and brain to impact.

\subsection{Methodological Developments}

Experiments evaluating the response of the brain following an impact to the head are the primary source of validation data for countless numerical and computational finite element (FE) models, which are actively used in injury mitigation. The validity of these models hinges on the fidelity of data obtained through experimentation. Data is derived from the collection of brain motion through visible and radiographic image capture, kinematic response, pressure response, and qualitative observations. Figure 1 outlines the typical quantitative measurements collected in these studies. Over time, understanding of the in vivo condition has improved, placing significant importance on the boundary conditions surrounding head impact. Early experimentation relied heavily on animal studies, which paint an imperfect picture of the response of the human brain to impact. Animal models lack the size and complexity of the human brain and do not provide the necessary boundary conditions for a direct human comparison [27, 28]. These studies did, however, allow new technical methods to be explored before eventually moving on to cadaver studies, which are the current standard. In addition to biofidelity improvements, data collection methods have become more advanced, increasing the temporal and spatial resolution of images used in motion analysis; rates and quality of kinematic and pressure measurements have also improved. These continued methodological advances have refocused the understanding of the implications of head impacts and resulting traumatic brain injuries. 


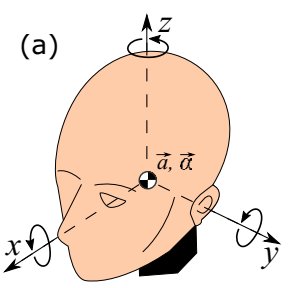

(b)

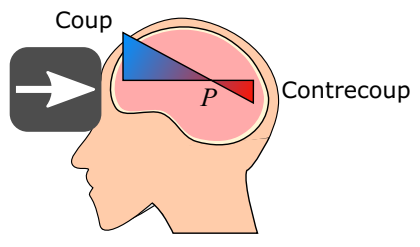

(c)

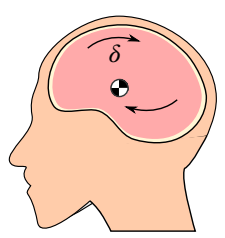

Figure 1: Quantitative measurements typically collected in impact studies. a) Kinematics, linear and angular acceleration with respect to the CG of the head. b) Pressure gradient in the intracranial space surrounding the brain, measured at the coup and contrecoup sites. Impact denoted by boxed arrow.

c) Displacement of the brain tissue with respect to the skull.

\subsubsection{Kinematic Data Collection}

Much of the early work in understanding head impacts and their survivabily comes from crash investigations and road safety efforts from the United States National Highway Traffic Safety Administration (NHTSA), who sought a metric by which vehicle crash safety could be measured and compared. Experimentation with animals and cadavers showed clear correlation between linear acceleration and injury survivability [29], due in part to skull fracture. The development through the 1960s of the Wayne State Tolerance Curve (WSTC) (Figure2) provided the foundation of what would become the Head Injury Criterion (HIC), a survivability scale of head impact, based on anterior-posterior linear acceleration, which was adopted by the NHTSA [10]. The HIC calculation results in a score, which is used to determine the crash worthiness of vehicles. The calculation is per:

$$
H I C=\left[\frac{\int_{t_{1}}^{t_{2}} a(t) d t}{t_{2}-t_{1}}\right]^{2.5} \cdot\left(t_{2}-t_{1}\right)
$$

, where $t_{1}$ and $t_{2}$ are the start and end of the time interval that will maximize HIC and $a$ is the linear acceleration measured in units of gravity $(g)$. 


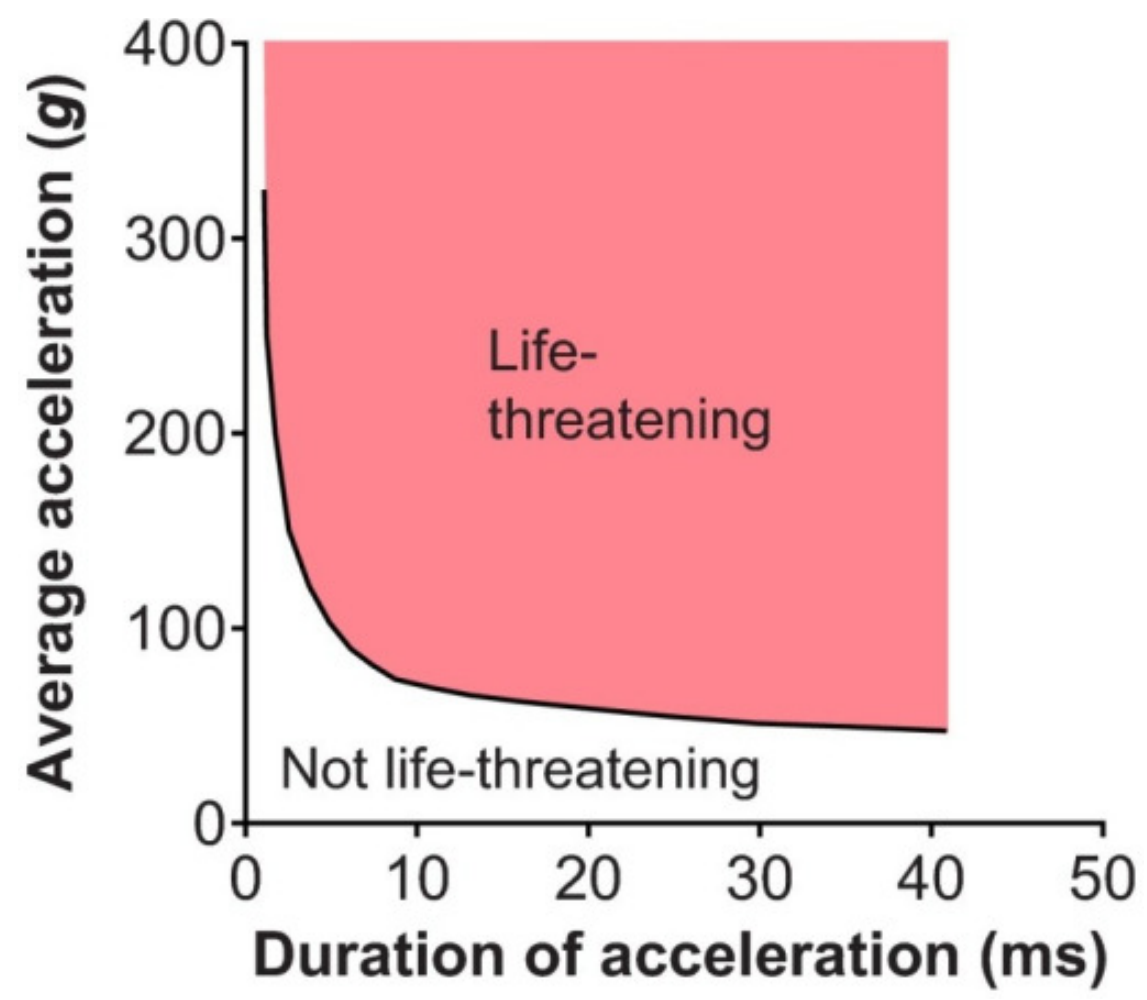

Figure 2: Wayne State Tolerance Curve. The curve depicts the survivability of head impacts, suggesting extreme accelerations (measured in units of gravitational acceleration) are survivable for very short durations. Reproduced from Namjoshi (2013) [30]. 
As a result of this interest in correlating linear acceleration to brain injury, kinematic measurement has been broadly incorporated and considered in both animal and PMHS impact studies [22,25, 31-36]. Overwhelmingly, it is understood that the kinematic input is not the cause of injury, rather it is the deformation and motion of the internal structures of the brain that result in trauma [31,32], and kinematics can only be used as an input factor in the assessment of potential outcomes. Further, it has been observed that complex resultant linear and angular acceleration must be considered when evaluating the response of the brain, as linear acceleration alone cannot sufficiently describe the loads applied to the head and brain [33]. Despite acceleration not being the direct cause of traumatic brain injury, it remains the best externally measured metric for the comparison of injurious head impacts and loading scenarios. Further, three dimensional kinematic inputs are used for the wealth of finite element and injury models in use today [13-[15]. As such, recording the complete kinematic response of the head following an experimental impact, or in recent studies, the directly applied kinematic impulses [35.36], is necessary to determine the internal response using FEM.

The quality and breadth of kinematic measurements has improved with technological advancements. Early studies incorporated the use of head-mounted linear accelerometers [31,32]. Given the quality and capture rates possible at the time, the ability to reliably resolve complex three dimensional motion came with the development of the 9-accelerometer array [37]. The 9-accelerometer array uses a 32-2-2 setup, as shown in Figure 3 , and has been incorporated into several PMHS impact studies [22, 25, 33]. Unlike a six-accelerometer arrangement, this method does not require the differentiation, and angular acceleration can be computed directly, making it more suitable for impact studies, especially when precision and capture rate of sensors is low [37]. Though possible to acquire a complete threedimensional motion profile from as few as six accelerometers, this method requires 


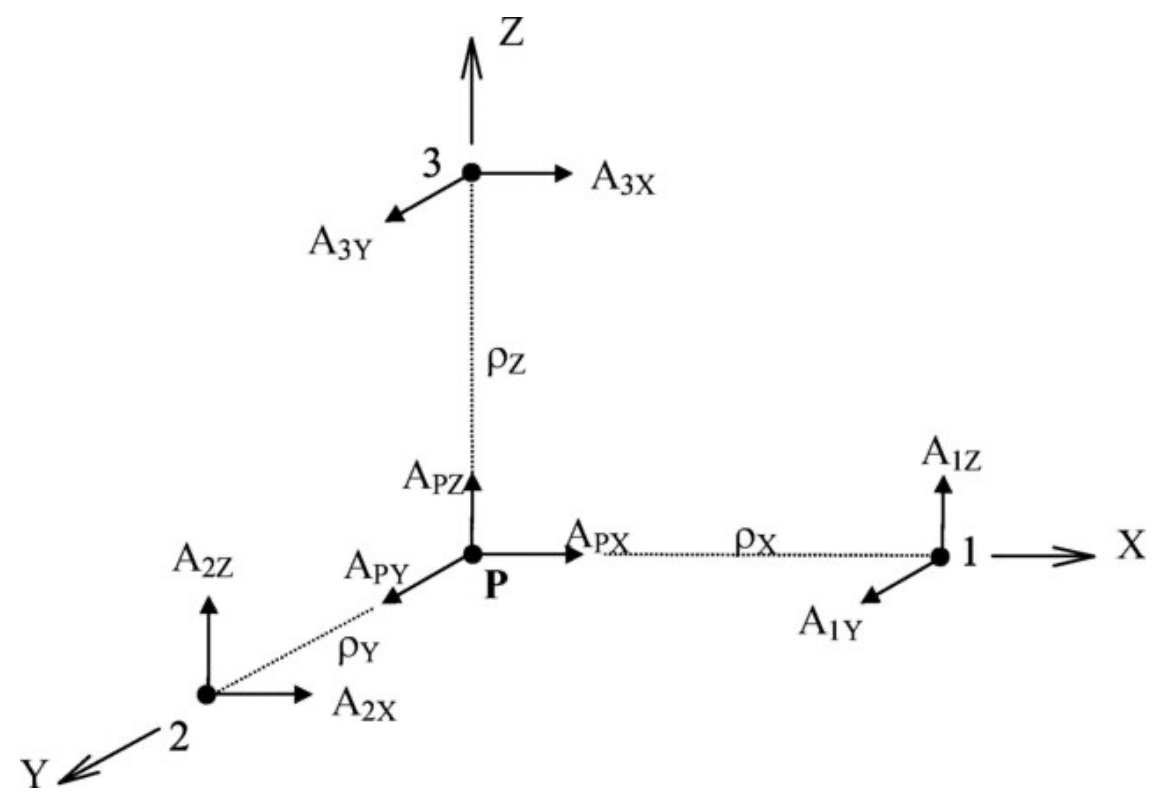

Figure 3: The 9-accelerometer array, as developed Wayne State University [37]. Using only linear accelerometers, the complete linear and angular acceleration of a rigid body can be computed, with minimal error. A central point holds three accelerometers, $\mathrm{AP}(\mathrm{X}, \mathrm{Y}, \mathrm{Z})$, while pairs of accelerometers are positioned in each plane at a known distance, $\rho$, from the point, $\mathrm{A} 1(\mathrm{Y}, \mathrm{Z}), \mathrm{A} 2(\mathrm{X}, \mathrm{Z})$, $\mathrm{A} 3(\mathrm{X}, \mathrm{Y})$. Unlike other methods that rely on time-based differentiation, this method is suitable for impact studies. Reproduced from Martin (1998) [38].

a high degree of precision and fast data acquisition rate in order to compute the angular acceleration, as the time derivative of angular rate is required. More recently, due to advances in sensors and data acquisition rates, angular rate acquisition has been used to compute angular acceleration [35, 36].

Even though the kinematic measurements alone cannot fully describe head impact events, correlations between input kinematics and injury have been developed and used in injury risk assessment. Efforts to incorporate measurement devices into protective equipment have helped to identify some impact regimes and subsequent symptomatically described injuries. Notably the Head Impact Telemetry (HIT) System (Simbex, Lebanon, NH) has been used for the capture 
of impact data for on-field evaluation of American football, as well as other impact sports [39]. This system incorporates a unique arrangement of accelerometers and impact sensors, and can transmit data in real-time to a computer, such that potentially injurious impacts and accelerations can be identified and logged. Attempts have been made to correlate the recorded impact data and post-impact symptoms with the intention of developing a better understanding of the threshold for injury. Unfortunately, errors associated with these devices, the limited ability to rigidly couple devices to the subject, and subjectivity around injury reporting has resulted in limited success [40]. Given these limitations, lab-based reconstructions of the impacts observed in sporting injuries that include kinematic and deformation measurement remain the optimal way to develop high fidelity model validation data.

\subsubsection{Pressure Measurement}

In efforts to identify and characterize internal modes of injury, intracranial pressure dynamics involving in-tact PMHS heads were first explored by Nahum et al., [41]. For a series of frontal impact, pressure was measured near the impact site, as well as opposite, at the contrecoup location. Piezo-resistive pressure transducers were mounted such that they could measure pressure below the dura, while not being so deep as to interfere with the PMHS brain tissue itself. From these studies, intracranial pressure was found to correlate linearly with head acceleration, with peak pressures occurring before significant motion of the head could be observed. In subsequent computer models, pressures were equated and compared to stresses in the brain tissue, using the empirical data for validation. The key findings were that the brain provides a damped response to motion. Though compressibility of tissues and fluids are often considered negligible, this should not 
be conflated with being incompressible. Similarly, brain tissue should not be considered incompressible, as it transmits non-trivial pressure waves. Inertial effects lead to negative contrecoup pressures, and pressurization of PMHS specimens results in the same in vivo response seen in earlier animal studies. Pressure data has served as a touch-stone for numerous additional studies [22, 23, 25, 33, 35] which have collected pressure using similar methods, allowing comparisons to be drawn across data sets, while serving as a form of experimental validation.

\subsubsection{Observing Brain Motion}

In early efforts to understand brain motion caused by head impacts, Pudenz et al. [19], used high speed cinematography in order to observe motion patterns of the cortical surface of the brain following impact in living rhesus macaque monkeys. The brain was made visible by removing the calvarium section of the skull and replacing it with a clear acrylic (Lucite) cap [19]. Their findings of brain motion following impact reinforced the understanding of coup-contrecoup injury mechanics, as well as the notion that concussive injuries were likely linked to impactinduced motion of the brain. This work was, however, not specifically focused on brain injury, but aimed to develop a method to investigate the brain under in vivo conditions, highlighting the need to consider boundary conditions in future brain injury investigations, as well as the value of high-speed image capture.

The concept of X-ray cinematography was introduced shortly after the discovery of X-rays, with the notion that body function and its response to external stimuli could be better observed using rapidly captured radiographic images [32]. A viable method of capturing the internal workings of the brain and skull following an impact was later implemented by Hodgson et al. [31] using flash X-ray cinematography. In this method, rapid X-ray exposures were made possible by 
discharging capacitors through X-ray tubes, aligning a radiographic film cassette with the observation area, and rapidly advancing the film with each exposure. This early study captured the deformation of a dog skull and subsequent brain motion following a direct blow to the head. High-speed flash X-ray exposures were used in numerous subsequent studies [22, 32, 33]. In these later experiments, fluoroscopic techniques were used in conjunction with rapid X-ray exposures. Instead of using radiographic film to record images, a high-speed film camera was used to capture the screen of an image intensifier, as outlined in Figure 4 In capturing the visible light output from the image intensifier, higher speeds could be achieved than with radiographic film systems. Complications existed in early iterations of this cineradiography system, resulting in a limited 200 frames per second (fps), as ghosting of images would build up on the intensifier screen. This was overcome through the use of a "rapidly erasing" image intensifier, boosting image capture to $1000 \mathrm{fps}$. With extremely short exposures of 30 to $50 \mathrm{~ns}$ per frame, motion blur could be eliminated, allowing fine details of small structures to be seen, resolving diameters as low as $300 \mu \mathrm{m}$ [32], provided appropriate contrast agents were used. Limitations of X-ray penetration through the dense skull and brain, combined with low image clarity due to the high-speed capture rate, has made it necessary to augment the contrast of the brain. Due to its high attenuation, Hodgson et al., used lead solder cut into 0.1 inch long strips and inserted them into the brain with a hypodermic needle [31]. Concerned about the possible migration of the lead markers, low-speed manipulations of the head were performed between image exposures, and compared. These manipulations showed that some markers were likely lost in fluid-filled regions of the head, while those captured in tissue tended to stay in place. Recognizing the potential for marker migration during higher energy impacts, and the notable increase in density over the surrounding 


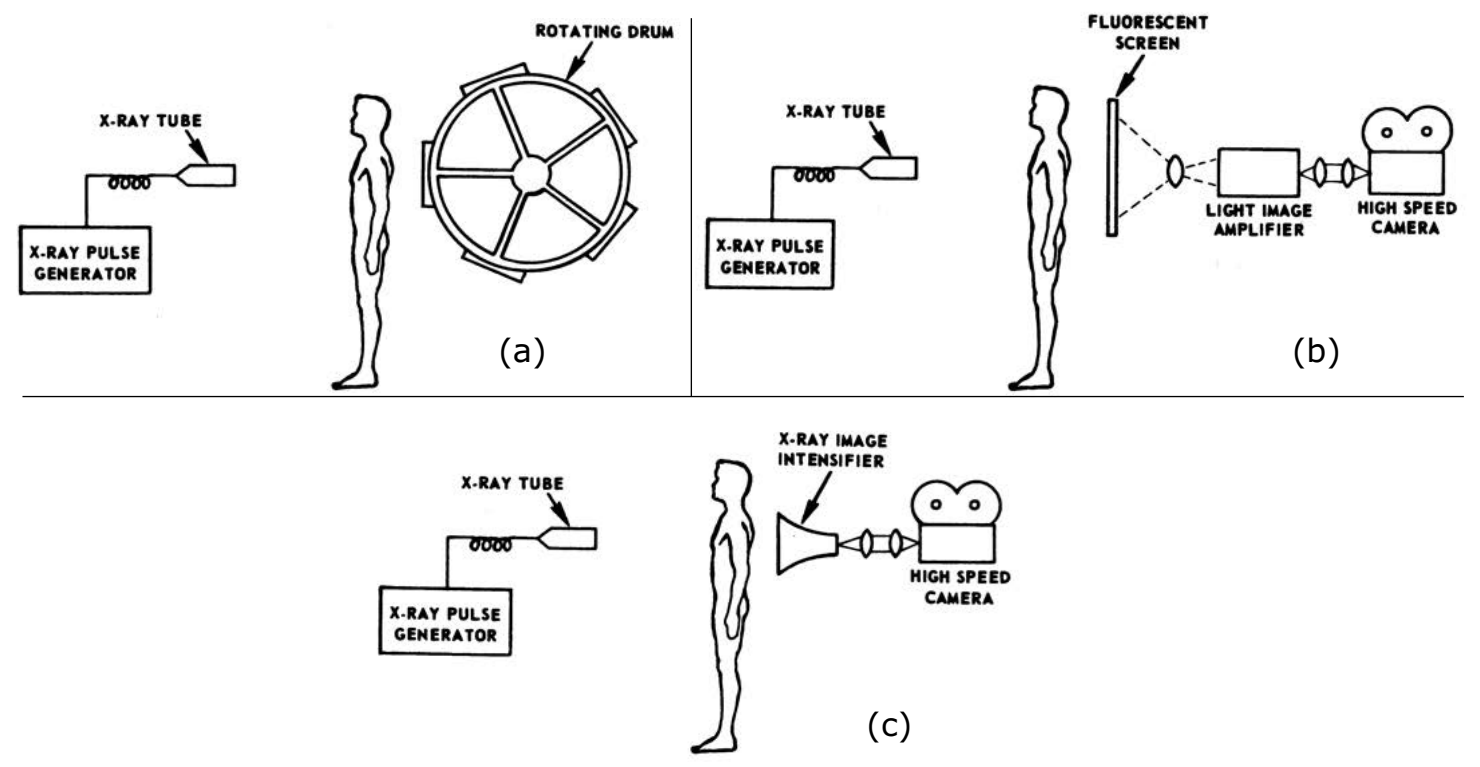

Figure 4: Flash X-ray techniques images and description adapted from Shatsky (1973) [32]. a) Simplest method of flash X-ray cinematography, sequential exposure of radiographic plates. Drum is representative of any mechanical device used for film transport. b) Cinefluorographic method, capturing a fluorescent screen with a motion camera. Optional image amplifier used to improve brightness. c) Most efficient (at the time) design of an X-ray cinematography system, incorporating an image intensifier, which combines a screen and image amplifier. 

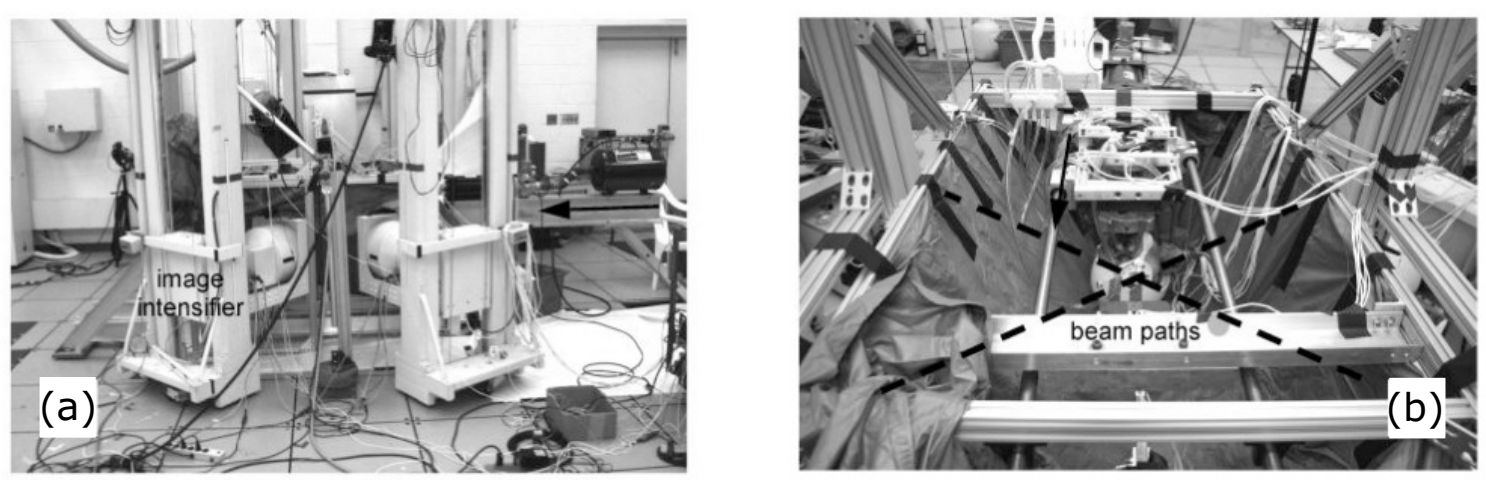

Figure 5: High speed bi-plane X-ray system, as developed by Hardy et al., [23] (a) Full system showing image intensifiers used for image capture. (b) Experimental set-up within image capture region, showing beam paths. Reproduced from Hardy (2007) [25].

tissue, alternative contrast agents were used in future studies. Shatsky et al., performed impacts with Macacca mulatta monkeys [32]. Monkeys were injected with radiopaque fluid (thorium dioxide), allowing observation of the motion of vascular systems. The study showed that that following a non-lethal head impact against a static anvil at $5 \mathrm{~m} / \mathrm{s}$, intracranial vasculature underwent small, rapid deformations. A similar study by Nusholtz et al. [22] made use of lines of radiopaque gel, inserted into the tissue of the brain of Rhesus monkeys and human cadavers. The position of the lines of gel was compared to known points on the skull to determine how the tissue deformed. Although it could be seen that the motion of the brain lagged the skull motion, limitations in the repeatability of gel placement and deformation of the gel lines within the brain following impacts made it difficult to fully develop an understanding of local deformation. Understanding these limitations, it was proposed that a bi-planar system, comprising two orthogonal cineradiography systems, could be capable of resolving 3-dimensional brain motion, though it would be a challenge to develop [32].

In efforts to develop validation data for analytical tools, models, and injury criteria, Hardy et al., developed a new protocol for capturing impact-induced brain 
deformation [34]. The new method incorporated elements and recommendations from previous studies [22,31-33], making use of a bi-planar imaging system utilizing high-speed fluoroscopic techniques, and improved, tissue embedded, neutral density targets (NDTs), along with high-contrast external reference markers. These investigations exclusively used the preferred human cadaver specimens, or postmortem human surrogates (PMHS). The biplanar system, as seen in Figure 5 , was operated at 1000 frames per second, and capable of tracking NDTs with $0.1 \mathrm{~mm}$ accuracy, made possible through modern high-speed digital cameras. The NDTs were developed with the goal of minimizing the effects of the markers on surrounding tissues, including inertial effects and migration, though little information exists on how these markers were validated. The NDTs, made from $1.9 \mathrm{~mm}$ diameter tin granules embedded in $2.5 \mathrm{~mm}$ by $5 \mathrm{~mm}$ polystyrene tubes, had an average density of $1.5 \mathrm{~g} / \mathrm{mL}$, closely approximating that of the surrounding tissue. In their first studies [24], NDTs were inserted in two separate columns, one posterior, and one anterior. Later [25], a three dimensional approach was taken, incorporating markers in two groups of seven, making the six verticies of an octahedron, along with a seventh central marker. These two configurations are seen in Figure 6. The incorporation of discrete markers, over that of vascular or intracranial contrast [22, 32], allowed the motion tracking of the brain tissue itself. Limitations of the NDT markers included the possibility of their migration through tissue following impact, or interference with the natural tissue response. Despite the possible limitations, this method permitted the first quantitative measurements of brain displacement and estimates of strain, versus that of previous qualitative, whole brain observations. Recently, it has been suggested that the neutral density of embedded markers is not necessary, and a low-mass marker is sufficient to avoid interference with the tissue response [35], though limited evaluation of this has been presented. The computation of strain from early studies using discrete markers 

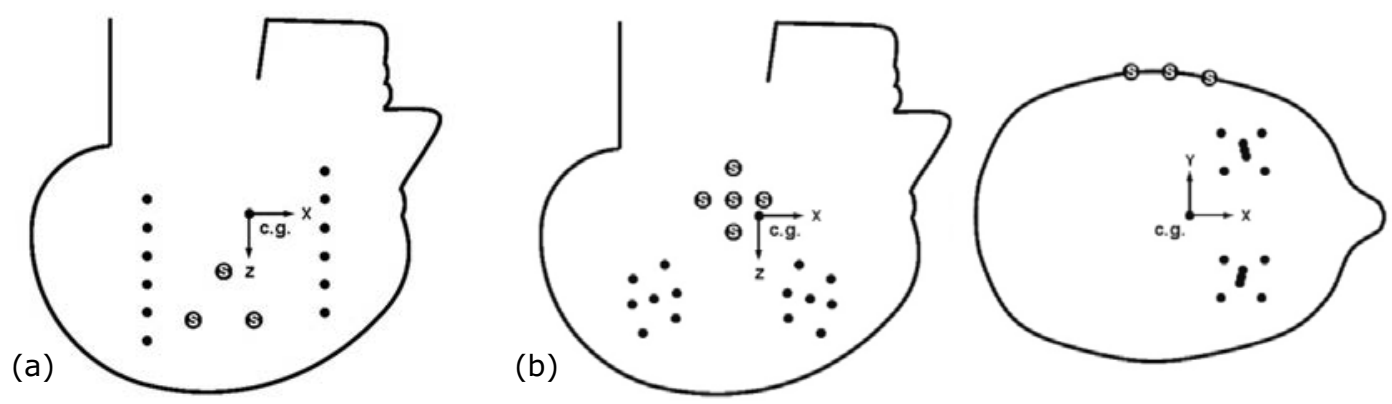

Figure 6: Positions of NDTs used in bi-plane studies. In each image, " $\mathrm{S}^{\prime \prime}$ denotes a fiducial marker placed on the skull. (a) Columns of NDTs placed in anterior and posterior regions in a single sagittal plane. (b) Sagittal and axial views of marker clusters at anterior and posterior positions. Clusters occupy a nonplanar volume of tissue. (a) Reproduced from King (2011) [42].(b) Reproduced from Hardy (2007) [25].

may not have been sufficiently described, resulting in a lack of clarity for the validation of FE and numerical models [18]. Rather than identifying brain displacement relative to the skull and calculating whole-brain strain from a broad group of tissue embedded markers, a more direct method would target the placement of markers to predetermined regions of interest, allowing direct regional strains to be computed following displacement tracing [18].

\subsubsection{PMHS Preparation}

Though beneficial in the search for understanding the response of the brain to impact, animal-based experimentation [19, 31, 32] does not provide validation data that can be used in model validation. Differences in brain and skull size, morphometry, and complexity make direct comparison of humans and animals infeasible [27, 28]. Except for a recent study involving low-intensity impacts [43], investigation in living humans is not possible, due to the risk of injury and lack of biocompatible measurement techniques. Given this, leading studies have centred around the use of PMHS [22, $25,33-36,41]$ to obtain the required empirical data. 
Recognizing the inherent limitations, PMHS impact testing has become the gold standard in generating this data, as it is believed to be the closest approximation of a living head and brain complex when specimens are adequately prepared [33, 41].

Preparation of the PMHS is time consuming, as anthropometric differences complicate mounting and instrumentation, while keeping specimens in tact requires careful handling. Given these complications, experimental set-ups can differ across sample sets, resulting in challenges drawing comparisons in data [41]. In order to move quickly upon the receipt of a specimen, apparatus and instrumentation that can be rapidly deployed and fit to a variety of specimens has been developed [22, 25, 33, 35]. As tissue tends to degrade following death, the timing of PMHS experimentation is sensitive. In early uses of unembalmed cadavers for crash and impact investigations, significant brain tissue degradation was identified in specimens greater than four days post-mortem [33], despite best efforts to preserve them at low temperature $\left(4^{\circ} \mathrm{C}\right)[22]$. More recent studies have suggested that primary degradation occurs within the first 24 hours post-mortem, allowing specimens to be stored at low temperatures for longer periods before experimentation begins [35]. Despite the concerns around degradation and specimen fidelity, reporting post-mortem duration has been limited in some studies [23],25], whereas others have begun preparations in as few as 14 hours post-mortem [36],

Validation data hinges on its ability to provide impact response measurements that mimic the in vivo conditions as closely as possible. Early comparisons between living animals and repressurized cadavers showed that the repressurization process allows for a close approximation to in vivo conditions [41]. Pressurization, repressurization and perfusion have been described interchangeably in the literature [22- 25, 33- 36, 41], and have referred to both cerebral and vascular fluid systems. Initially performed using complete cadaver bodies, vascular pressurization was achieved through a catheter inserted at the junction between the left and right 
carotid arteries [33]. Later, in addition to vascular pressure, cerebral pressure was achieved via a mid-spine tap into the spinal dura [22]. As the use of entire cadavers has shifted to the use of PMHS heads alone for these studies, a standard description of perfusion has been described [25, 35, 36], with fluid passed into the cranial space through the dural sheath and inlets fitted to the skull, while vascular fluid is flowed through the carotid arteries and jugular veins. Fluid reservoirs are elevated to provide continuous pressure of about $1 \mathrm{mH}_{2} \mathrm{O}$, with leaked fluid being recycled to a main reservoir [25]. Though these methods derived from the original full-cadaver studies have seen broad use, their efficacy has not been established, and some regions may contain trapped gases, reducing the biofidelity of the cadaver model.

Mounting PMHS heads into testing apparatus has become necessary as entire cadavers are now rarely used. No standard method of mounting the head has emerged, however alignment of the head with impact and rotational inputs is common across studies to restrict motion to the observation plane. In impact scenarios, the head has typically been suspended by the neck in an inverted configuration with the goal of achieving better perfusion [23. 25], though this may interfere with the natural response of the tissue. Studies using pure rotational inputs have clamped the specimen in place, confining rotation to a single plane [35, 36]. Examples of these methods can be seen in Figure7 7 . 

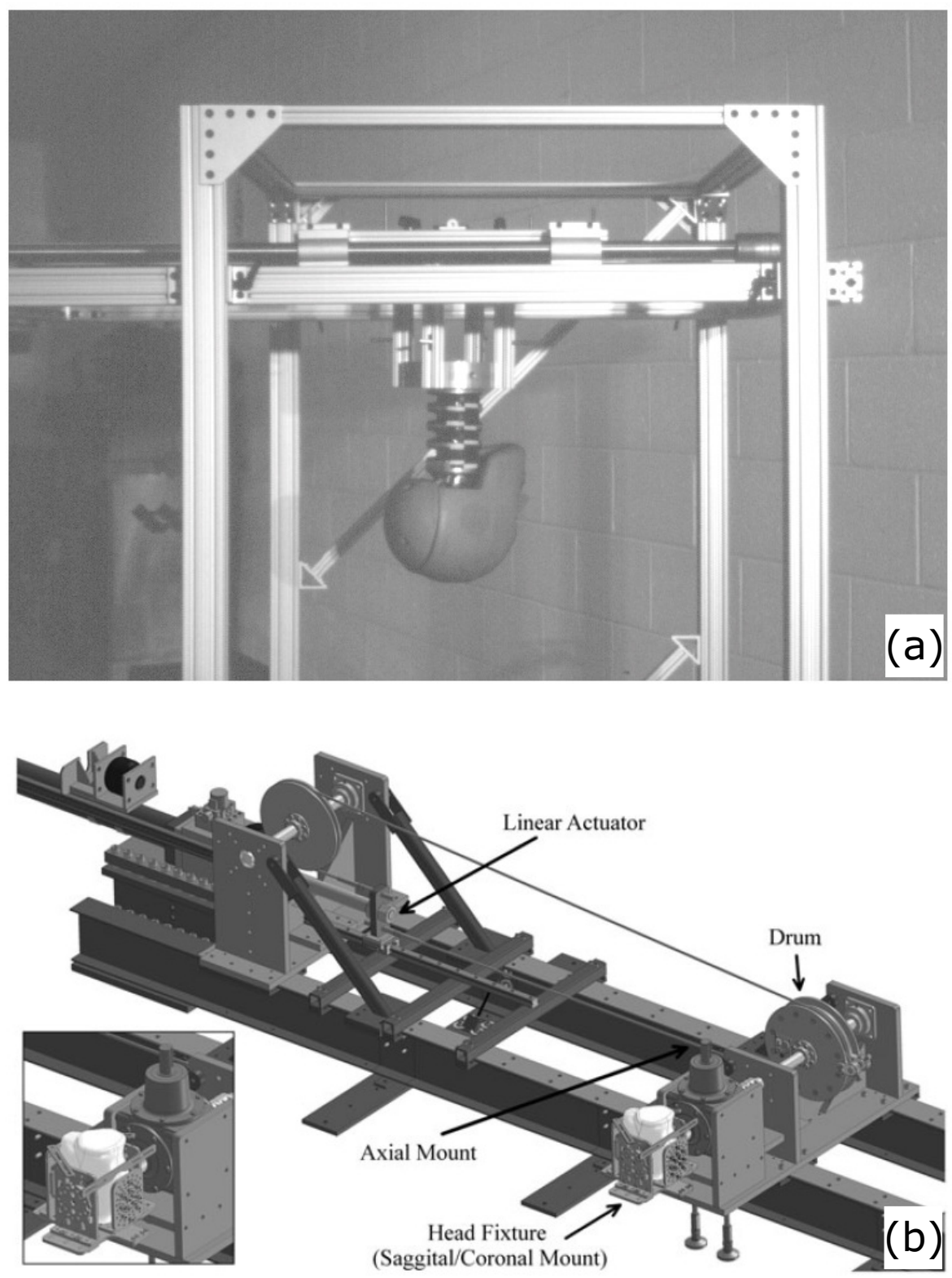

Figure 7: (a) From Al-Bsharat (1999) [23], inverted mounting of specimen inside translation frame. (b) From Alshareef (2019) [36], specimen contained within a clamp, attached to a single axis rotation drive. 


\subsubsection{Alternative Techniques}

As studies have continued to clarify the mechanical response of the head and brain to impact, experimental methods have moved towards improved temporal and spatial resolution in observing brain motion, while making great efforts to approximate in vivo conditions as closely as possible. Whereas the majority of the studies discussed earlier have followed similar methods; using PMHS to identify brain motion with radiographic imaging, while capturing input kinematics and intracranial pressure, two notable studies have attempted alternative techniques.

Aiming to provide validation data for a true in vivo response of the head to impact, Bayly et al. used magnetic resonance imaging (MRI) to identify motion in a single sagittal slice of the brain following low-intensity linear and angular accelerations [43] in three living subjects. Peak displacements observed were inline with previous observations [24, 31], while far greater spatial resolution was possible as the MRI technique provided a view of an entire slice of tissue, and not discrete embedded markers. Further, the lagging motion of the brain with respect to the skull and vortex-like rotation of the brain tissue proposed by earlier studies [24,25] were also observed, highlighting the occurrence of shear strain following impact. Although this study provides the greatest spatial resolution to date, and a true in vivo response, it is limited by a relatively low temporal resolution, as capture rates were approximately 179 frames per second. At this low sampling speed, high-speed responses may go undetected or be erroneously reported [18]. Responses before $5 \mathrm{~ms}$ following impact have been observed in other studies [25. 36. 41], where sampling rates of at least $1 \mathrm{kHz}$ are utilized.

In an effort to provide three dimensional validation data for whole-brain models, Alshareef et al. developed a novel method to capture brain motion without 
the use of imaging [36]. Using skull-mounted and tissue-embedded sonomicrommetry crystals, the response of the brain following pure rotation of the head was captured. Unlike image-based methods, the ultrasonic system directly measures the distance between crystals with high precision, allowing a more direct computation of tissue strain. In addition, to aid in the localization of observed strains across different structures, CT imaging was used to pinpoint the position of the crystals following their insertion in the tissue. Measured displacements were similar to those from previous studies [25], though impact characteristics may not be identical as only rotation was considered. While this method is capable of evaluating three dimensional whole-brain deformation, the crystals must be connected via signal wires to data acquisition equipment, and although the crystals were not seen to migrate through tissue, their presence, along with the signal wires, may inhibit the natural motion of the tissue.

\subsection{Overview of Key Findings}

Ongoing head impact investigations have revealed a great deal of information about how the brain responds and deforms during an impact event. Early qualitative studies provided limited understanding of the motion and twisting of the brain at its surface due to its complex structure [19]. With the ability to observe the whole brain using flash X-ray techniques, the lag between brain and skull became more apparent, and internal deformation was confirmed [31]. The improvements to methods and incorporation of kinematic measurement along with X-ray observation connected injurious levels of acceleration to injury mechanics [32], though specific injury mechanisms were unmeasured.

Quantitative measurements have enabled the development of simulations and 
models, which have informed estimations and assumptions surrounding brain injury modes. Pressure measurements at, and opposite from, an impact site, identified a linear relationship between pressure and acceleration, illustrating that pressure gradients through the tissue may cause significant injury. Peak coup pressures ranged from 25 to $500 \mathrm{kPa}$ at the impact site and contrecoup pressures from -5 to $-85 \mathrm{kPa}$ for impacts with resultant linear accelerations of 30 to $400 \mathrm{~g}$ [41]. Peak pressure was seen to significantly lead the motion of the head, underscoring the compressibility of the brain [41]. . Additional studies of impacts at speeds of 2.7 to $3.6 \mathrm{~m} / \mathrm{s}$ have shown similar pressures at the impact site of $140-150 \mathrm{kPa}$, but contrecoup pressures approaching $-165 \mathrm{kPa}[23]$ seen in these studies are unrealistically low and may be erroneous; pressure transducers are not typically calibrated for these low pressure measurements. Furthermore, limited evidence of cavitation due to low pressure exists [25]. The relationship between impact speed and resultant acceleration depends on impact location and impactor material, however impacts of $3.5 \pm 0.3 \mathrm{~m} / \mathrm{s}$ showed resultant linear accelerations ranging from 38 to $291 \mathrm{~g}$ [25]. Improvements in imaging allowed the measurement of tissue displacement with respect to the skull, as well as the motion paths of tissue deep regions. Following impact and rotation, tissue tends to move in figure-8 patterns, with reduced displacement closer to the centre of gravity [24, 25, 35, 36]. In impacts up to $3.6 \mathrm{~m} / \mathrm{s}, 2-3 \mathrm{~mm}$ of relative displacement has been observed in deeper tissue structures, while at the interface between the brain and skull, larger displacements of 9-11 mm have been observed [23,25]. In pure rotation studies with angular accelerations between 1200 and $3400 \mathrm{rad} / \mathrm{s}^{2}$, relative brain-skull displacements have been reported between 3 and $5 \mathrm{~mm}$ [35]. The pressure response was reduced in the pure rotation study, to a range of $-28 \mathrm{kPa}$ to $42.5 \mathrm{kPa}$, as no coup or contrecoup locations exist without an impact site. 


\subsection{Summary}

Investigation of traumatic brain injury relies on use of validated injury models and computational simulations. The data derived from PMHS impact studies, including kinematic, pressure, and motion analyses, has been used extensively in model validation [13-15]. Advances in empirical data collection methods to date have improved the quality of models available for simulating impacts and evaluating injury mitigation schemes. Collection of pressure and kinematic data in cadaver impacts was instrumental in the development of early FE models of the brain [41]. Pressure data remains the primary mode of validation of empirical methods for more advanced image-based studies [22,23, 25, 33, 34], which have permitted some validation of displacement-based models. In more recent studies [35, 36], impacts have been replaced by purely kinematic impulses, as rotation has been of particular interest. Although these experiments have given further insight into the deformation of the brain due to rotation, they do not tend to replicate true head impact characteristics. Despite these efforts and advancements, three dimensional, in vivo impact response data, capable of evaluating the strain across structures of the brain does not exist.

In order to further develop the data available for model validation and advancement of the understanding of traumatic brain injury, specifically with respect to tissue strain, additional empirical studies must be designed and performed [18]. Considering the limitations of the PMHS, efforts to closely approximate the in vivo condition must be made. Minimization of the error introduced by the measurement technique is paramount. In studies using embedded markers, particular care and validation of the tissue response must be taken with respect to the mechanical and dynamic coupling of the markers to the tissues. Further, the location of markers in the tissue must be clarified and identified in order to evaluate the response 
of discrete structures in the brain, as the heterogeneous nature of the brain must be considered. The following chapters of this thesis will examine improvements and developments made by the author in HSXR image processing, embedded tissue markers, and a PMHS methodology designed to improve biofidelity. With these developments, direct computation of discrete structures strain may be possible, aiding in the development of advanced models, simulations, and surrogates. 


\section{Chapter 3}

\section{Image Capture and Processing}

The development of a robust set of data showing how strain propagates through the brain tissue after a head impact requires high quality tissue displacement data. Direct measurement of brain tissue displacement is a challenge, as it is optically inaccessible, and to expose the tissue such that it could be viewed optically would alter boundary conditions beyond a reasonable degree. Fortunately, capturing images of internal anatomy is made possible through a broad range of diagnostic imaging modes, including radiography (X-Ray), magnetic resonance imaging (MRI), and ultrasound. While these technologies may provide clear images of distinguishable anatomical regions in the brain, they lack the necessary temporal resolution to resolve the motion of these features during impact events [23]. Temporal resolution can be dramatically improved through the use of high-speed X-ray (HSXR) cinematography [23]. The improved temporal resolution comes at the cost of spatial resolution and a loss of signal-to-noise ratio in resultant captured images. These challenges can be overcome through the insertion of passive contrast targets into the regions of interest. This approach is similar to that used in radiostereometric analysis (RSA) which has been used for gait studies [44, 45]. In gait analysis, strain rates are relatively low along with inertia, meaning that the mechanical properties of the markers are not a concern. The development of a 
calibrated, low-influence contrast marker is discussed in Chapter 4. Displacement data is extracted from the images captured in by the HSXR system through a series of post-processing methods. The experiments outlined herein were performed using the HSXR facility at Carleton University.

\subsection{High Speed Cineradiography at Carleton}

The HSXR facility at Carleton University was developed primarily to service the needs of a PMHS head impact study. The apparatus components were carefully selected to facilitate the capture of brain tissue motion at 10,000 fps and an 1megapixel image resolution. The system captures and stores images in 12-bit grey-scale, providing 4096 discrete contrast levels. A range of spatial resolutions $(\mathrm{px} / \mathrm{mm})$ can be obtained using this system, depending on the desired observation region, as focal distances can be adjusted to magnify specimen projections. The HSXR facility was used to collect experimental data for both the development of embedded PMHS contrast markers, and the PMHS impact experiments. These studies are outlined in greater detail in Chapters 4 and 5 , respectively. An overview of how the system was set up for each of these studies is described below. Figure 8 is provided to illustrate the spatial arrangement of the HSXR and other experimental apparatus.

\subsubsection{Use in Marker Development}

The development of contrast markers for use in the PMHS study did not explicitly require the use of the HSXR system. The purpose of the study was to determine if, and how the mechanical properties of embedded markers affect the deformation of the surrounding matrix. In the case of this study, the surrounding matrix was 


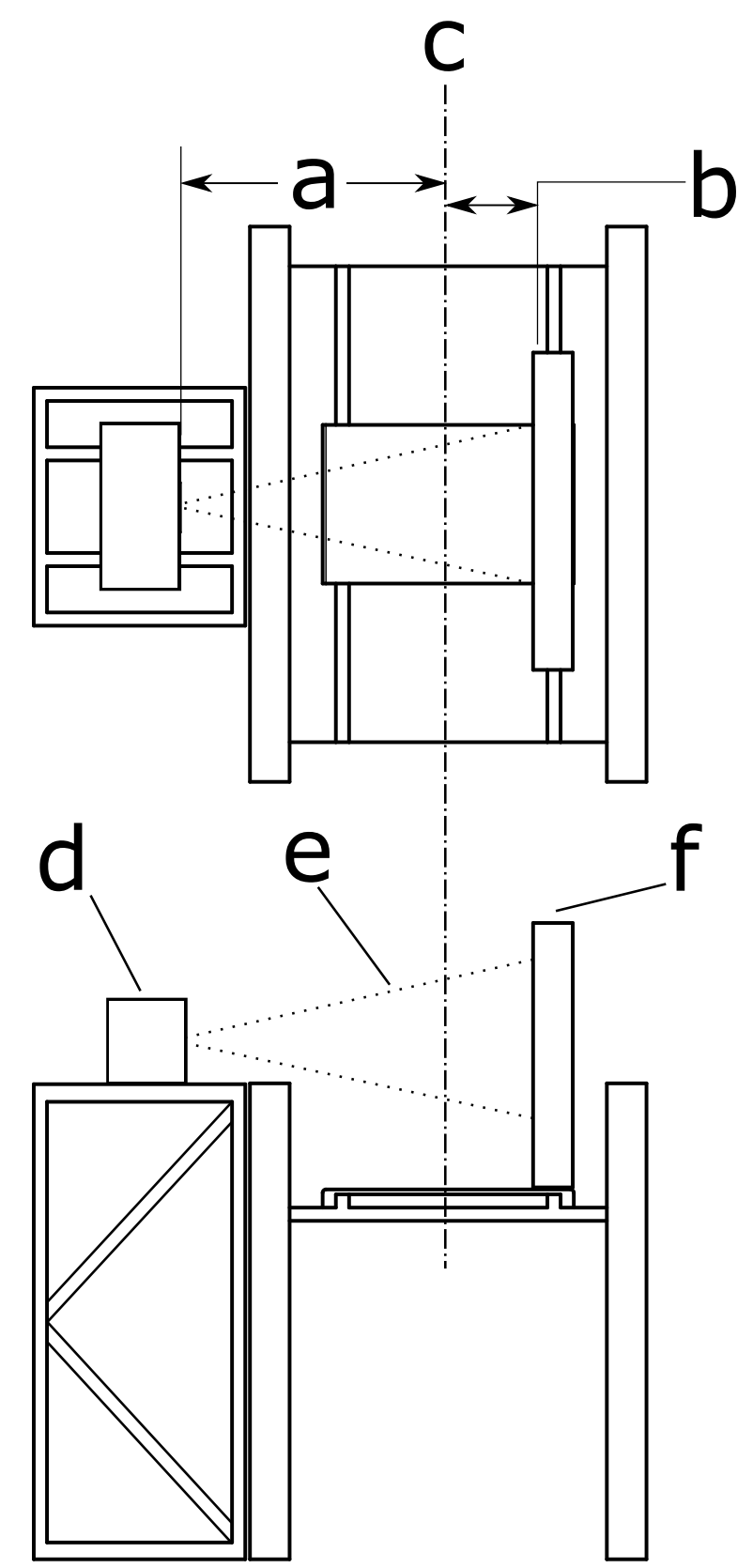

Figure 8: Overview of HSXR System. a) Distance from source to imaging plane. b) Distance from imaging plane to projection surface. c) Imaging plane. d) X-Ray source. e) Incident X-Ray cone beam. f) Detector. 
optically clear, allowing the deformation to be captured with a visible light camera. The HSXR system was ultimately used as it provided additional details on the relative contrast between marker designs, which was useful in selecting the final marker for the PMHS study.

Relating to Figure 8 , the drop-tower was placed between the X-ray source and observation region in a position chosen to minimize distortion effects. The distance from the source to the specimen (a) was $70 \mathrm{~cm}$, and the distance from the specimen to the detector (b) was $20 \mathrm{~cm}$. The final impact position of the drop-tower was centred vertically with the X-Ray source to limit cone-beam and vignetting artefacts. These placements resulted in a pixel-to-millimetre scale of $0.25-0.35 \mathrm{~mm} / \mathrm{px}$, allowing clear and defined sub-millimetre measurement of particle displacements. X-Ray power was set to $100 \mathrm{kV}$ and $500 \mathrm{~mA}$ and a duration of $630 \mathrm{~ms}$, providing sufficient penetration, clarity, and time for the entire impact response to be observed. Captured images were stored as 8-bit monochrome TIFF, containing both the specimen and its housing, with a contrast range suitable to distinguish the target markers from the surrounding matrix. A capture rate of 7,500 frames per second was used, which resolved motion at the final impact speed of $3.9 \mathrm{~m} / \mathrm{s}$, or $0.5 \mathrm{~mm} /$ frame. Two steel fiducial markers, required for data extraction, were made from $50 \mathrm{~mm} \times 50 \mathrm{~mm}$ flat bars and fixed to the outer housing of the specimen such that they did not interfere visually with the motion of the specimen. These features are highlighted in Figure 9 


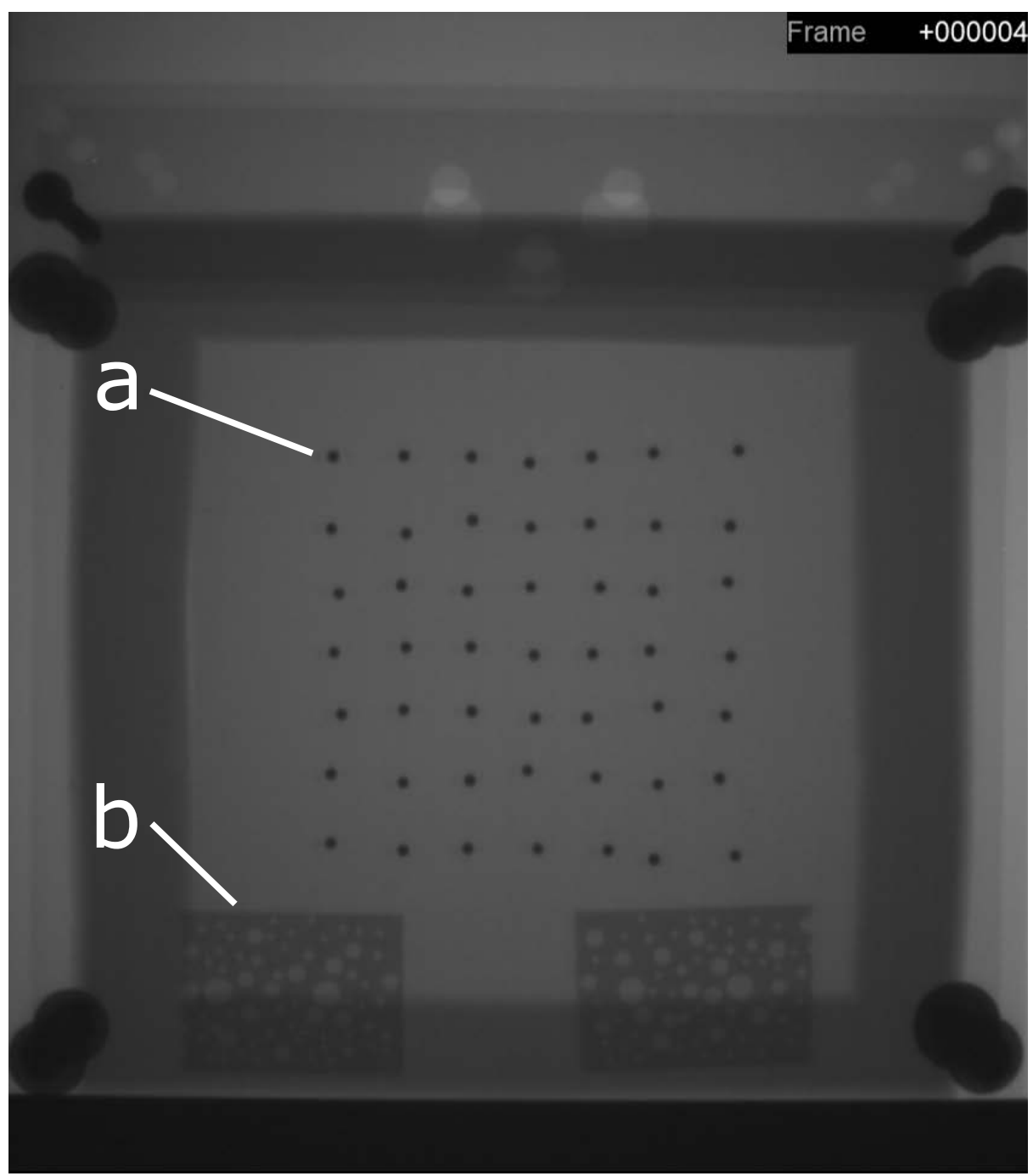

Figure 9: Resultant image from HSXR as used in marker study. a) Experimental contrast marker group. b) Fiducial markers used for stabilization. 


\subsubsection{Use in PMHS Study}

The PMHS specimens were placed in the HSXR observation area in an upright orientation, with tubing and wiring for supporting apparatus (perfusion, kinematic and pressure measurement) connected to an overhead elastic cable, pulling these components out of the observation area. The position of the specimen was measured from the plane containing the embedded contrast markers, as capturing motion of the the markers was the primary objective. The projection surface and specimen were adjusted in the vertical direction to align with the impact site under investigation. The line of action of the rigidly mounted linear impactor was used as a centreline, as its position was not adjustable. The projection surface and Xray source were then adjusted further to minimize distortion effects and artefacts. Referring to Figure 8 , the distance from the source to the marker plane (a) ranged from $60 \mathrm{~cm}$ to $80 \mathrm{~cm}$ and the distance from the marker plane to the projection surface had a range of $10 \mathrm{~cm}$ to $20 \mathrm{~cm}$, depending on the specimen size. A calibrated steel ruler was placed in the observation area, outside of the specimen, but within the marker plane. Still images captured with the ruler in place provided a reference for evaluating the pixel-to-millimetre scale for each specimen at precisely the marker position. Resultant scales were varied across the sample set, due to the varying impact conditions and specimen geometries (Appendix D).

X-Ray power was set to a level such that contrast markers were optimally visible, while anatomical features, including the ventricular system, remained identifiable. A capture time of $630 \mathrm{~ms}$ was sufficient to capture the impact event and subsequent lagging tissue motion. Images were stored as 12-bit monochrome TIFF, providing 4096 discrete contrast steps. Impact motion was captured at a rate of 7, 500 frames per second, enabling smooth marker tracking through the entire impact event, which is paramount in the computation of tissue strain. Light-weight, 


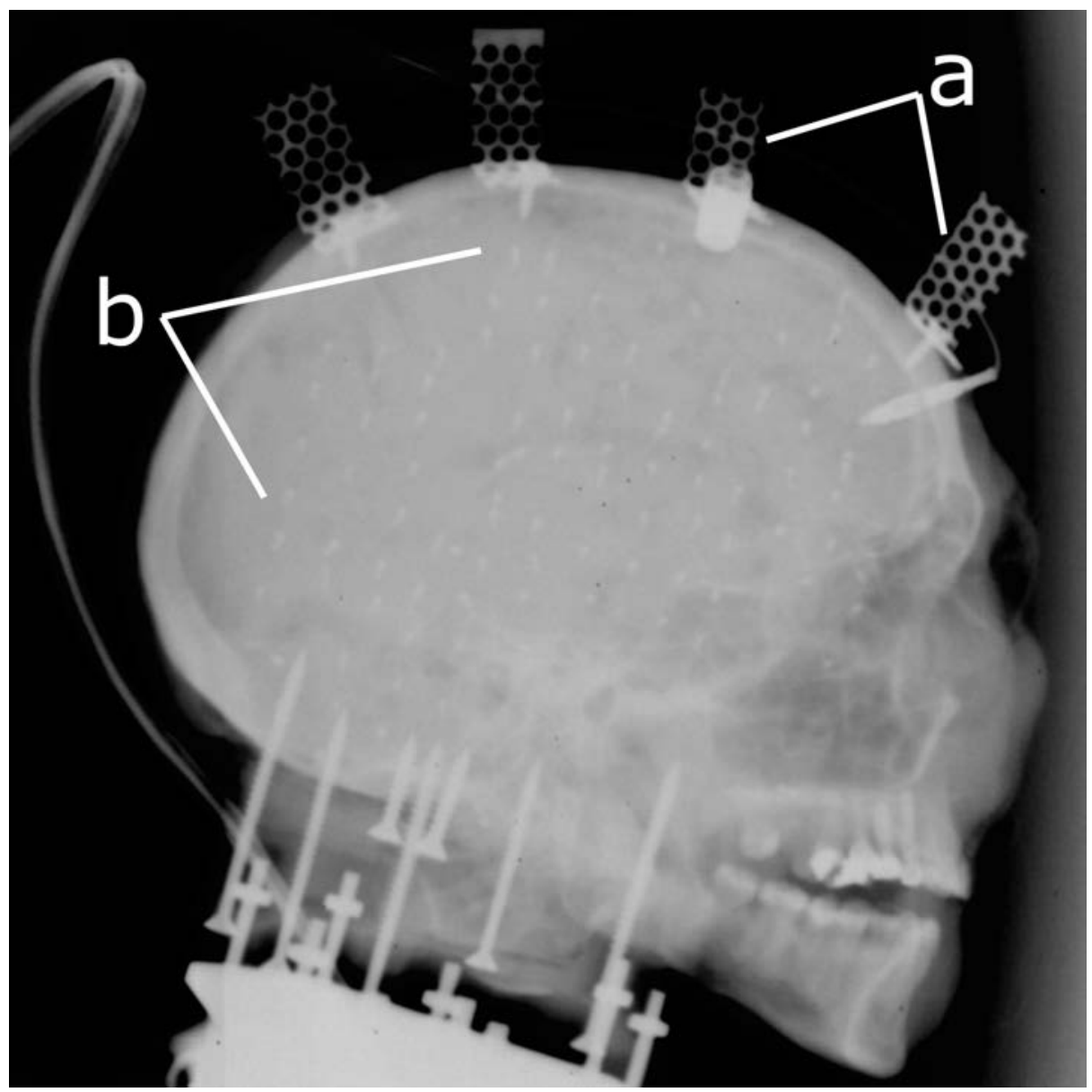

Figure 10: Resultant image from HSXR system from PMHS study, image inverted and contrast adjusted for printing clarity. a) Fiducial markers used for stabilization. b) Target contrast markers.

high contrast fiducial markers were fixed to the specimen in the marker plane to ensure that the images could be processed accurately and efficiently. Features of the images can be seen in Figure 10.

\subsection{Data Extraction}

The high data capture rate used in the impact studies outlined herein yielded a sizeable amount of raw data. For example, a single impact could easily produce 
over 500 images, each of which may contain more than 50 target markers, resulting in 25,000 data points. Although it would have been possible to manually track the targets and develop displacement paths, automation dramatically reduced the time requirement and human error associated with this type of data extraction. A number of existing software packages were tested for image processing and marker tracking purposes, including MOSAIC [46] and TEMA (Image Systems $\mathrm{AB}$, Sweden), however due to associated costs and limited output formats, they were not chosen for the project. Instead, the extraction of target position data from images collected in the marker development and PMHS studies was made possible through custom-written, automated post-processing code in MATLAB, developed by the author of this thesis. Primary lens distortion correction was performed as a batch process on all images as per the camera lens parameters, removing erroneous curvature. Following primary correction, in-house processes included clarity improvements, stabilization, and target tracking. Each process was validated to reduce or eliminate additional error in the measurements. As images were acquired from the system in a regulated time series, the extracted target positions could be assembled into individual displacement paths, and further differentiated spatially to compute strain.

\subsubsection{Clarity Improvements}

Features of interest captured in the images acquired by the HSXR system exhibited a range of clarity. Features with weak edges or local noise were not always suitable for algorithm-based data extraction, as pixel-to-pixel intensity differences are a key measure in identifying features. As images are radiographic projections, their clarity is based on the X-ray attenuation through the different materials present in the 
specimen. Adjustments to incident X-ray energy can improve image clarity, however this was not always the case, as these adjustments result in a balance between contrast for brightness [47]. At times, certain features were only detectable through qualitative assessments and visual inspection of the images. A qualitative method was not feasible for automated software, which relies on quantitative measures for feature detection. Improving the clarity of the features increases the edge-quality, making target detection more effective and efficient [47].

The most commonly used post-processes to improve clarity for data extraction was a simple contrast adjustment, as performed by the built-in MATLAB function imadjust. This function maps image intensity values from a specified range to the maximum range available in the image format. An example of this process is seen for an 8-bit image in Figure 11. In the example, the random noise in the background has a pixel intensity of $169<I<255$, which is visually similar to that of the target markers in the foreground, with a pixel intensity of $I=168$. Contrast adjustment is possible by remapping the marker intensity $(\mathrm{I}=168)$ across the entire 8-bit spectrum allowing the intensity, and thus the features of interest to stand out clearly.

Much like a spotlight, the X-ray source does not project an even field. Image intensity tends to be greater toward the central focal spot, resulting in less intensity at the periphery [47]. This produces a vignetting effect in the acquired images, reducing the brightness and clarity of the features in the far-field regions. In addition to basic contrast adjustment, flat field correction was used to reduce the effect of the vignetting, helping to clarify image features in shaded regions. This process was performed using the MATLAB function imflatfield, which smooths the image intensity using a Gaussian filter, estimating the gradient intensity and 


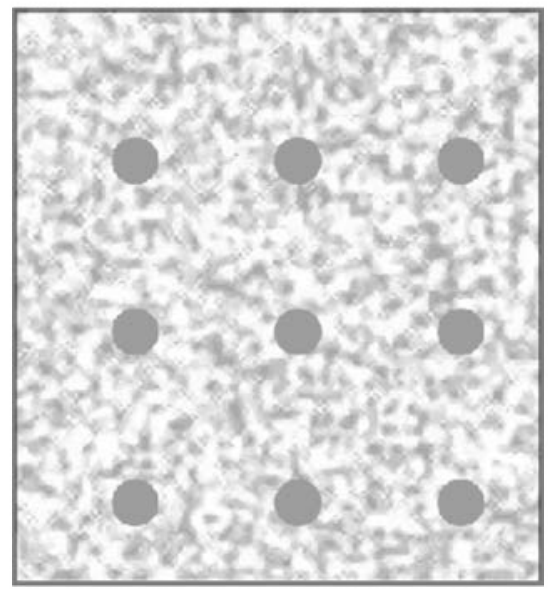

a

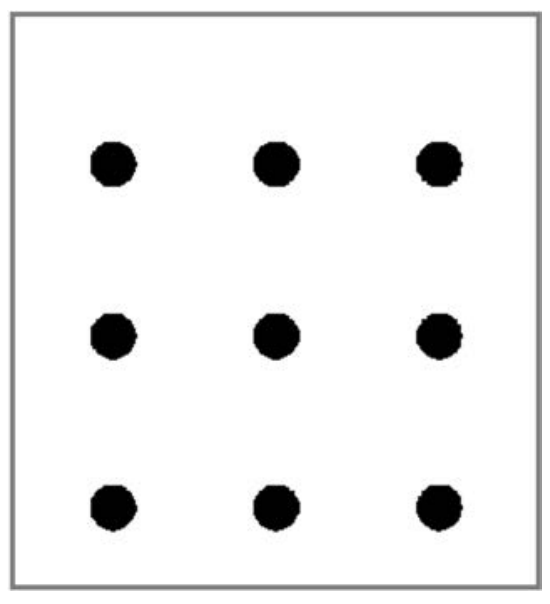

b

Figure 11: Example of contrast adjustment. (a) Original image containing target markers in regular grid, and random intensity noise in background. (b) Contrast adjusted image with background noise removed.

reversing its effects. The size of the Gaussian filter is specified through a userprovided standard deviation. For the processes used in this work, a standard deviation $\sigma \approx 0.25(\mathrm{G})$ was used, where $G$ is the estimated intensity variation provided by the gradient. An example of this process is shown for an 8-bit image in Figure 12. In the figure, a gradient is introduced across the sample at an arbitrary angle to simulate the vignetting effect. A flat field correction is applied with $\sigma=30$, followed by a contrast adjustment, mapping $145<\mathrm{I}<150$ across the entire intensity range. Combined, these processes allowed the marker features to be detected clearly by the feature recognition algorithms used in displacement tracking. 


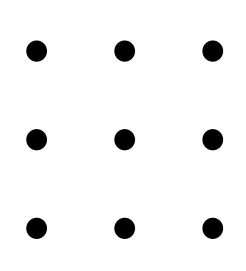

Signal

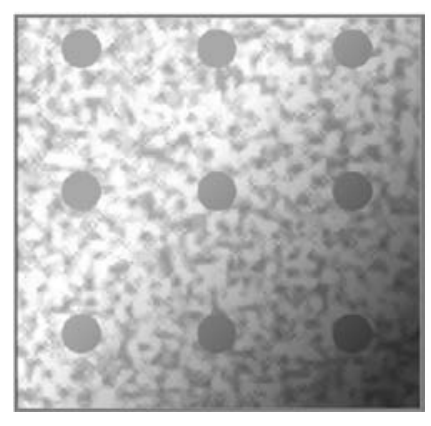

a

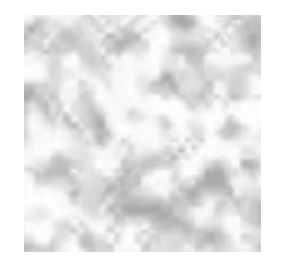

Noise

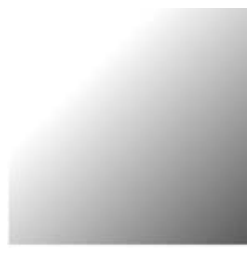

Vignette

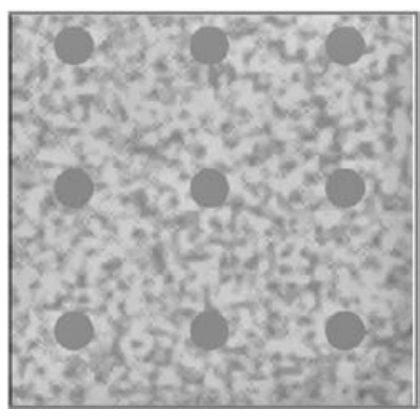

b

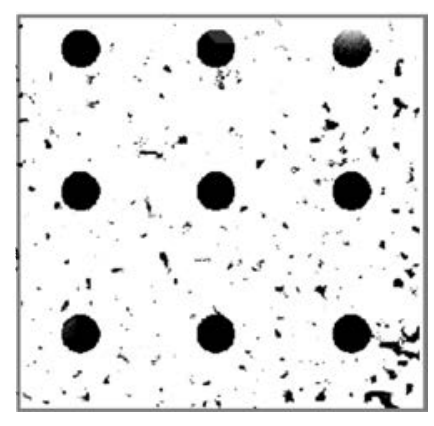

C

Figure 12: Flat field correction example. (a) Original signal image with random background noise, target markers in regular grid, and vignetting due to gradient overlay. (b) Flat field corrected image, minimizing effects of gradient. (c) Contrast adjusted final image used for particle recognition. Remaining noise is ignored due to spatial requirements for target markers in tracking process. 


\subsubsection{Stabilizaiton}

The image capture system records the motion of the specimen from a fixed, Eulerian, frame of reference, making it a challenge to separate rigid body motion from the internal material deformation. To simplify the data analysis, an image stabilization algorithm was employed using customized scripts and functions from the MATLAB Computer Vision Toolbox [48]. In this process, rigid-body-connected fiducial markers are identified with a user-selected region of interest in the first video frame. The strongest features of the fiducial makers are identified, and their positions stored. The automated process continues for all subsequent video frames, tabulating the feature positions across the entire observation period. Following the position tracking, a non-reflective similarity transform is computed for each frame,

$$
\left[\begin{array}{l}
x^{\prime} \\
y^{\prime} \\
1
\end{array}\right]=\left[\begin{array}{ccc}
S_{x} \cos \theta & -S_{x} \sin \theta & S_{x} t_{x} \\
S_{y} \sin \theta & S_{y} \cos \theta & S_{y} t_{y} \\
0 & 0 & 1
\end{array}\right]\left[\begin{array}{l}
x \\
y \\
1
\end{array}\right],
$$

where $(x, y)$ is the feature position in the original frame, $\theta, S_{x}, S_{y}, t_{x}, t_{y}$, are the respective rotation angle, scaling factors, and translations between the first frame and instantaneous frame, making up the transform parameters, and $\left(x^{\prime}, y^{\prime}\right)$ is the position at the instant of observation.

These transforms are used to rotate, translate, and scale each video frame to match the position of the fiducial features to the first video frame. It is noted that scaling can skew the relative position of points, resulting in an inaccurate transformation. As such, scaling was limited to $0.05 \%$ per frame to limit this error. The complete transformation of all video frames resulted in a change in the frame of 
reference from Eulerian to Lagrangian, allowing internal deformation to be observed clearly. The process is outlined visually in Figure 13. The method was validated by capturing the motion of a pendulum swinging through the observation area. Metallic hardware components with strong X-ray attenuating properties were adhered to the pendulum, to provide high-contrast, crisp fiducial markers. The results of this process are seen in Figure 14. Although any rigid-bodyconnected features, such as bolts or screws, could be used as fiducial markers for the stabilization process, later experiments utilized purpose-built fiducial marker plates. These plates were designed with crisp, high-contrast, randomly distributed features for efficient identification. 


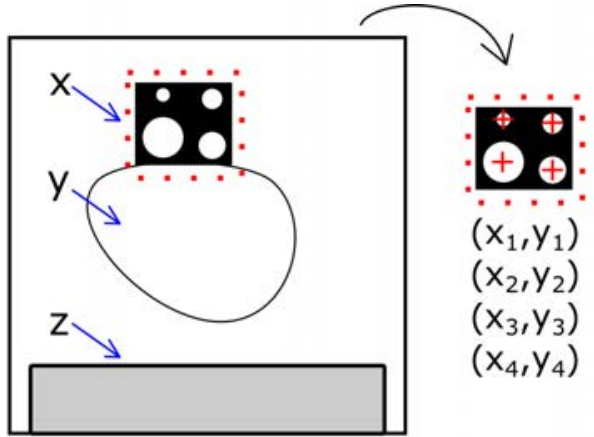

(a)

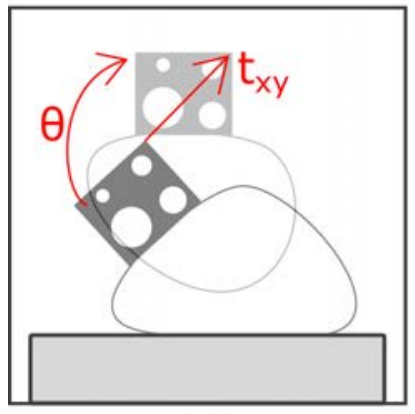

(c)

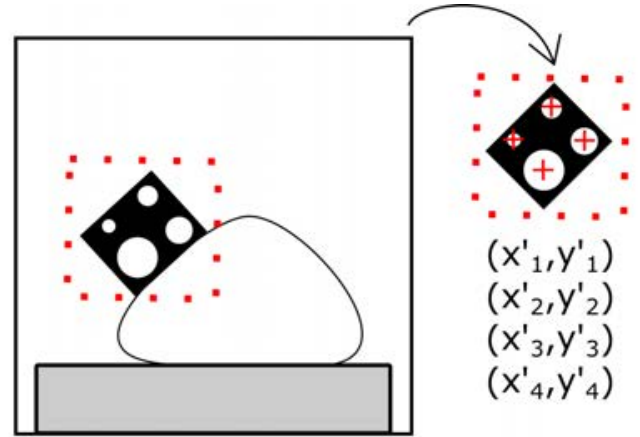

(b)

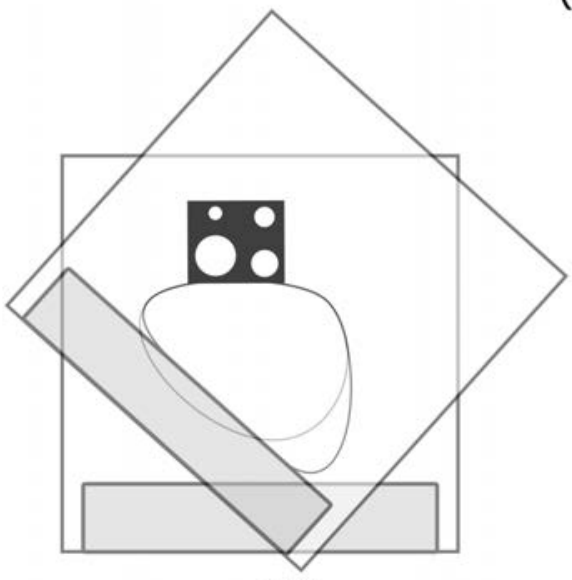

(d)

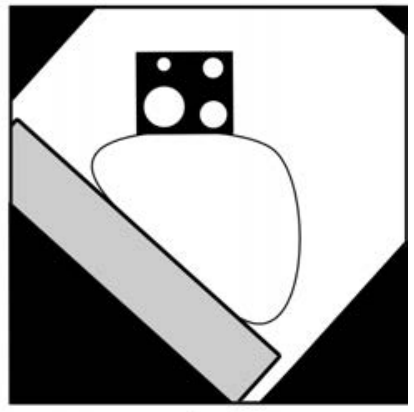

(e)

Figure 13: Overview of stabilization process. Fiducial marker $(\mathrm{x})$ is highlighted with user-selected ROI. Marker is attached to deformable object (y), which will impact anvil (z). (a) Features of fiducial marker are identified and coordinates stored. (b) Features of fiducial marker are identified and coordinates stored separately. (c) Transform between coordinates is computed. (d) Image in (b) rotated and translated to position in (a). (e) Image is cropped to fit original frame size and saved, allowing deformation to be observed clearly. 


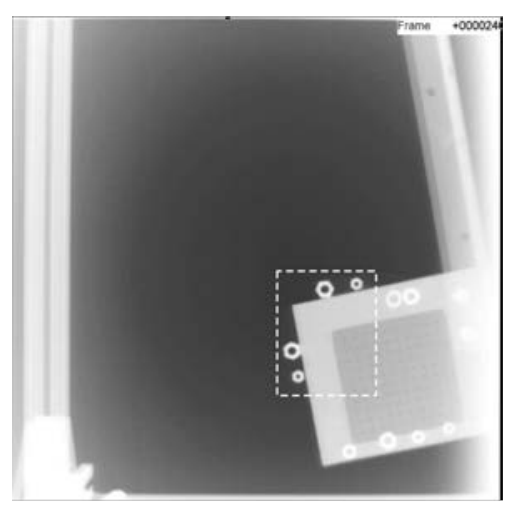

(a)

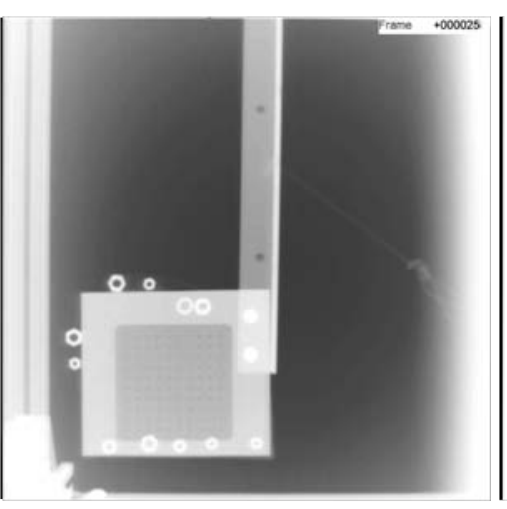

(b)

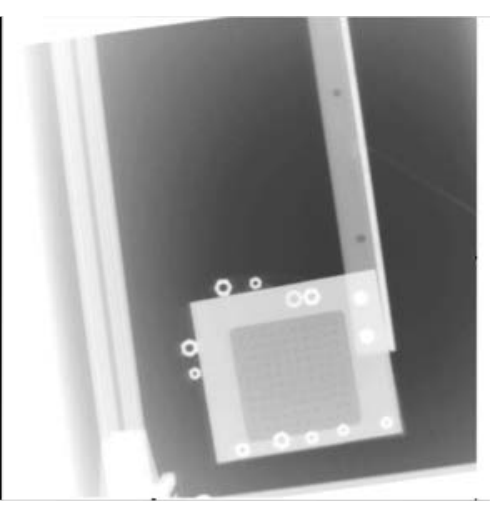

(c)

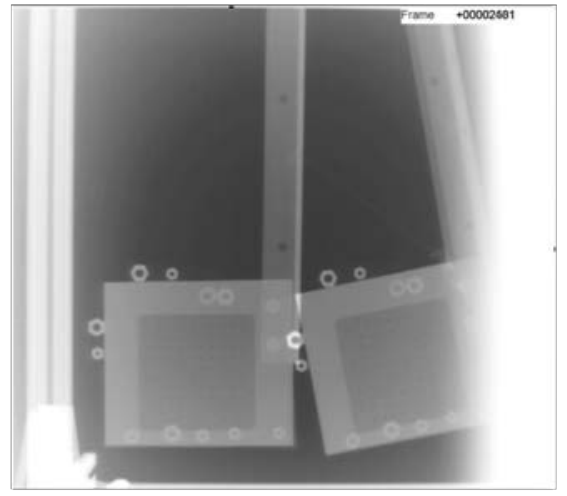

(d)

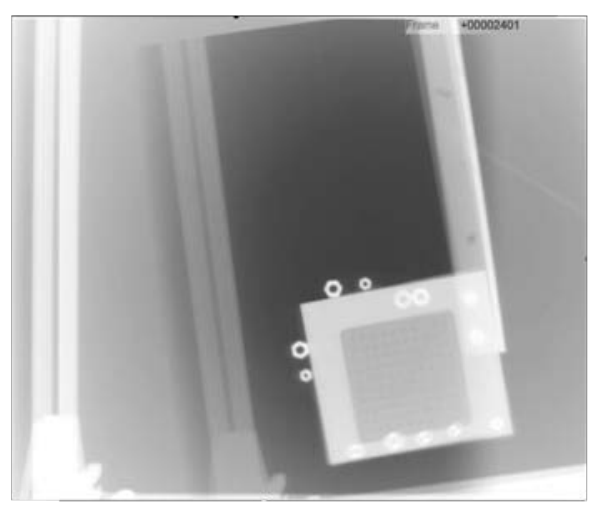

(e)

Figure 14: Validation of stabilization method, images contrast-adjusted and inverted for clarity. (a) First frame in pendulum swing with pattern ROI highlighted. (b) Mid-swing position from camera reference frame. (c) Mid-swing position from pendulum frame of reference. The video frame has been rotated and translated to match the first frame.(d) Composite image of $\mathrm{A}$ and B. (e) Composite image of A and C. 


\subsubsection{Determining Target Displacement}

A custom particle tracking code was written to extract the marker displacements from the Lagrangian shifted images. The script implemented two methods to identify features for tracking, which could be chosen by the user. The first method incorporated the Binary Robust Invariant Scalable Keypoints (BRISK) feature detection algorithm [49]. The second method made use of centre of gravity (CG) identification, as described in the next subsection. For each method, image clarity adjustments as described earlier were performed automatically during the tracking process to help identify particle centres. In the first frame of the sequence, a user-scalable region of interest (ROI) was positioned around each of the target points to be tracked. This point selection process was controlled by the user, however targets were indexed automatically in order of their selection. Unlike other, more computationally efficient, particle tracking codes [46], this code focused on maintaining an indexed position history for each particle.

For each subsequent frame in the image sequence, BRISK features or particle CG were identified within each target ROI and the centre of each particle was computed and indexed in a time series. The process was completed for the entire video sequence, with the ROIs moving along with each respective particle. Following of particles was possible by maintaining an ROI size greater than the maximum frame-to-frame position change, ensuring that even a shifted particle would remain in the ROI, allowing its centre to be identified. If a particle could not be identified due to a lack of clarity, automatic image enhancements and ROI expansion were performed in an attempt to search for the particle. A later version of the tracking code used a linear fit of previous points in order to predict the future position of particles, aiding in ROI placement and algorithm speed. To reduce false-positives, if a particle was found beyond a pre-determined, user-defined 
pixel radius, user intervention was required to confirm the particle position. The final output of the tracking process was a multi-dimensional array containing the pixel positions of marker centres for each frame. This process is outlined visually in Figure 15 .

The output array was subsequently processed to determine the stepwise and total displacement of each target marker through the impact event. As bulk-body motion was eliminated in the stabilization process, the extracted displacement was representative of the deformation due to impact. Displacement was calculated by combining pixel-based output data from the particle tracker and applying the appropriate scaling factor to transform displacements from pixels to millimetres. The first-frame positions of each marker were used as initial positions, from which all total displacements were calculated. Similarly, frame-to-frame displacements could be calculated. 


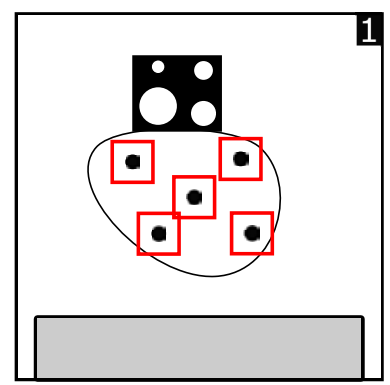

a

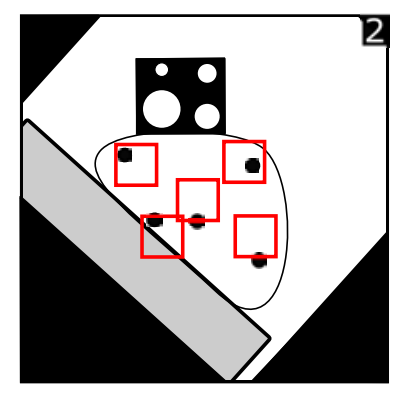

C

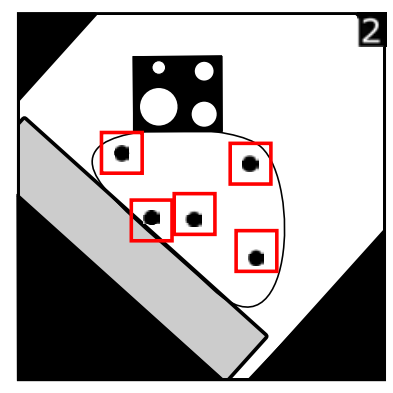

e

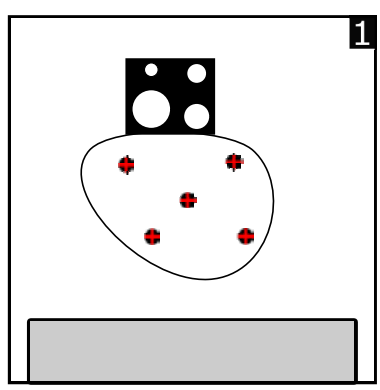

b

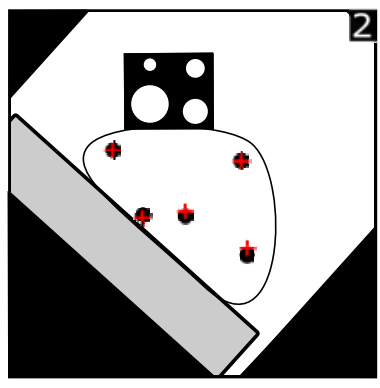

d

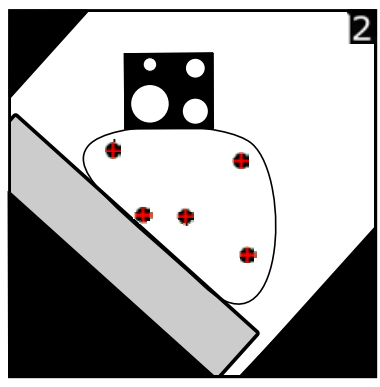

f

Figure 15: Outline of particle tracking process. (a) Particles in Frame-1 highlighted with user selected ROIs. (b) Particle centre identified using BRISK feature or CG detection. (c) Particle centres from Frame-1 used in Frame-2 to position ROI. (d) Particle centres estimated with new ROIs. (e) ROI positions adjusted to assumed particle positions. (f) True particle centre for Frame-2 identified using same method as in (b). 


\subsubsection{Centre of Mass}

Previous works have published impact-induced tissue displacements with respect to the centre of gravity (CG) of the specimen [25]. As such, estimation of the CG was necessary for the validate and compare the motion observed in the PMHS impact study. Additionally, the CG offset was used to transform the kinematic response from the accelerometer position to the CG of the head. A variety of methods exist to estimate the CG of the human head using external anatomical reference points and multi-axis balances [50]. Instead of these methods, radiographic projections were used to create a two-dimensional density map, in a simplified version of the CT method proposed by Roush [51].

Radiographic projections of the sagittal and coronal planes of the PMHS specimen were captured using a clinical imaging panel (Varex Imaging, 4343DXV) at a power level of $50 \mathrm{kV}$ and $80 \mathrm{~mA}$ for $250 \mathrm{~ms}$ using the HSXR source. Images were captured with a monochrome contrast depth of 16 bits, providing 65355 discrete levels of image intensity. The high-resolution (9-MP) panel permitted a pixel-tomillimetre scale of $8.2 \mathrm{px} / \mathrm{mm}$. With these images, clear anatomical details were visible, allowing tissues and regions to be distinguished from one another.

Images were processed using a custom MATLAB code, designed to compute the intensity-weighted centre-of-mass of any user-selected region of interest. Within the ROI, the intensity of each pixel was equated to the density of the material represented by the pixel, rendering the entire image an effective density map of the ROI. This simplifying assumption (equating density and pixel intensity) may introduce some error into the final determination of the CG. To overcome this, the ROI could be further subdivided to weight tissue layers separately (i.e., bone vs cortex), however this process was found to have minimal impact on the results, as 
the mass-attenuation of these materials is similar at the power levels used in imaging [52]. The resultant CGs for the PMHS specimens provided sufficient precision for kinematic transformations. When used for particle tracking in HSXR images, this method was found to estimate particle centres with a scatter of $0.7 \pm 0.1 \mathrm{px}$.

Considering that all pixels have the same area $(0.12 \mathrm{~mm} \times 0.12 \mathrm{~mm})$ and represent unit volume, the density prescribed by each pixel intensity allowed the mass of each pixel to be considered, making it possible to compute a balance point. Much like determining the equivalent load position in a distributed load system, the horizontal and vertical components of loading could be established by summing moments about a common point, and dividing by the total moment by the total load, to find the point-load equivalent position. For the horizontal coordinate, this is given by,

$$
\begin{array}{r}
m_{j}=\sum_{i=1}^{M} I_{i} \\
W=\sum_{j=1}^{N} m_{j}, \\
T=\sum_{j=1}^{N} m_{j} x_{j} \\
\bar{x}=\frac{T}{W}
\end{array}
$$

where $m_{j}$ is the sum of the intensity, $I$ of the vertical line of $M$ pixels from top to bottom. $W$ is the sum of sums for all $N$ lines of pixels, from left to right. $T$ is the total moment, about a common point, measured by $x_{j}$, across the image. Finally, the horizontal coordinate of CG is given by $\bar{x}$. The same procedure is followed for the vertical coordinate. 

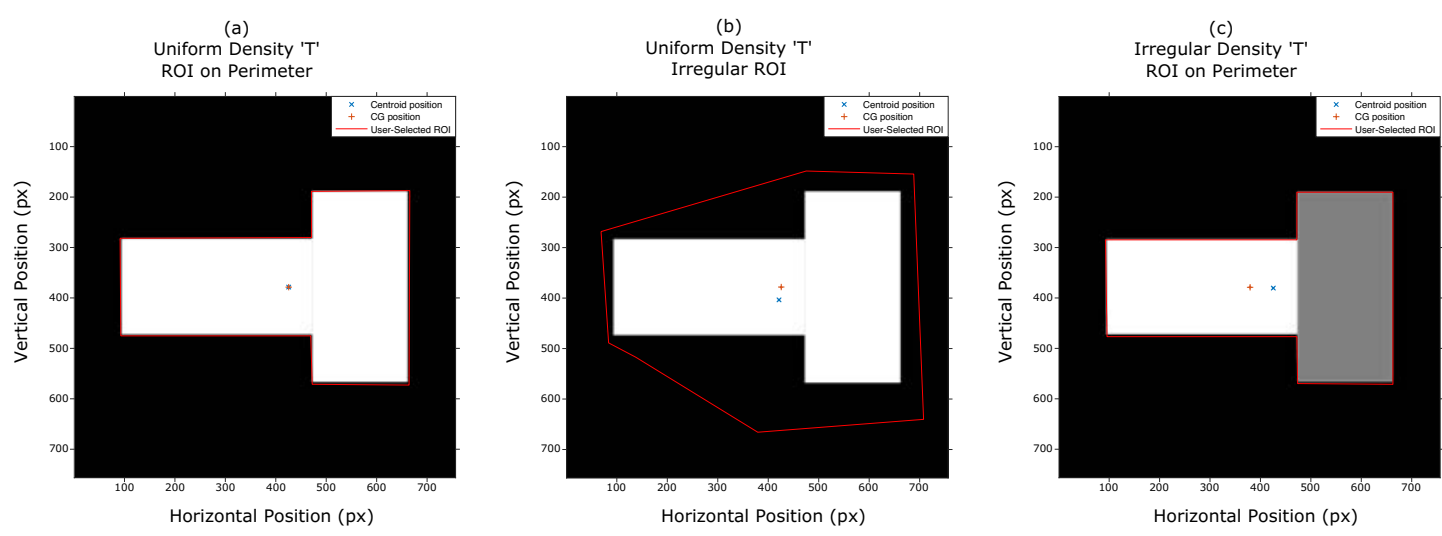

Figure 16: Estimation of $C G$ using test shape. (a) Uniform density ' $T$ ', with centroid (' $x$ ') and CG ('+'), ROI tight to shape perimeter. Centroid and CG coincident due to uniform density. (c) Uniform density ' $\mathrm{T}$ ', $\mathrm{ROI}$ irregular. Centroid corresponds to shape of ROI, whereas CG remains at correct point. (c) Mixed-density ' $\mathrm{T}$ ', ROI tight to perimeter of shape. Centroid and CG at correct, non-coincident positions.

This method was validated with computer generated phantom images. A sample of this output can be seen in Figure 16. Resultant coordinates of CG for PMHS specimens fall within the region approximated by external anatomical reference points. With the CG position marked, a reference scale could be used to localise its position relative to fiducial and target markers in distance units. 


\section{Chapter 4}

\section{Contrast Marker Development}

Collecting reliable validation data requires a well calibrated data collection system. A key element of the investigation of brain tissue motion using HSXR is the embedded contrast markers used to target regions of interest within the brain. As these markers may affect the response of the surrounding tissue, it is necessary to evaluate their influence on the observed measurement. While little has been published on the effect of marker properties on the measured response to impact, a recent study has highlighted the post-impact migration of markers [53] causing concern for the validity measurements obtained from hard, dense markers. This chapter outlines a sensitivity study performed in order to identify variability in dynamic coupling of embedded markers and a surrounding material.

The goal of this investigation is not to develop a perfect embedded marker, but to identify how the mechanical properties of markers may influence the observed response, and to narrow the design parameters for future marker designs. Additional marker properties, such as manufacturability, ease of insertion, and Xray attenuation must also be considered in the selection for PMHS studies. The present study compares differences in the impact-induced displacement response of a tissue-simulating material (neat material) to that of the same material with embedded markers. Tests were carried out at strain rates similar to those achieved 
in PMHS impact testing $\left(10^{1} \mathrm{~S}^{-1}\right)$.

A Taguchi L-9 orthogonal array was used to develop nine unique marker designs using three design variables, each at three levels [54]. The experimental marker design variables selected for investigation were density, stiffness, and pattern spacing. Each of these variables was tested at three values, corresponding to minimum, maximum, and mid-range quantities appropriate for PMHS impact studies. An additional three marker designs were used to evaluate and compare the new marker designs to the neat material and previous marker types [24, 33]. The markers were cast into a characteristic block specimen and dropped from a fixed height onto an impact surface to generate the displacement fields. The displacement response was used to evaluate the dynamic coupling between markers and matrix material. Multiple drops were conducted for each marker type to evaluate experimental scatter between individual experiments for a given marker design.

\subsection{Experimental Details}

Experimental markers were embedded and arranged in a grid pattern, along a single midplane of a tissue-simulant block (Medical Gel \#4, Humimic Medical, Greenville, SC), replicating the proposed pattern for the PMHS study. Gel blocks were cast within a mould measuring $152 \mathrm{~mm} \times 152 \mathrm{~mm}$ x $45 \mathrm{~mm}$ (Figure 17). Block dimensions were chosen in order to maximize the test-region given the limitations of the imaging and drop-testing systems available at the time of testing. A total of 15 blocks were created, one for each of the nine new marker designs, two for a massless marker, two for NDTs, and one for lead markers. The massless, ink-based markers were used to evaluate the response of the neat material. These massless 


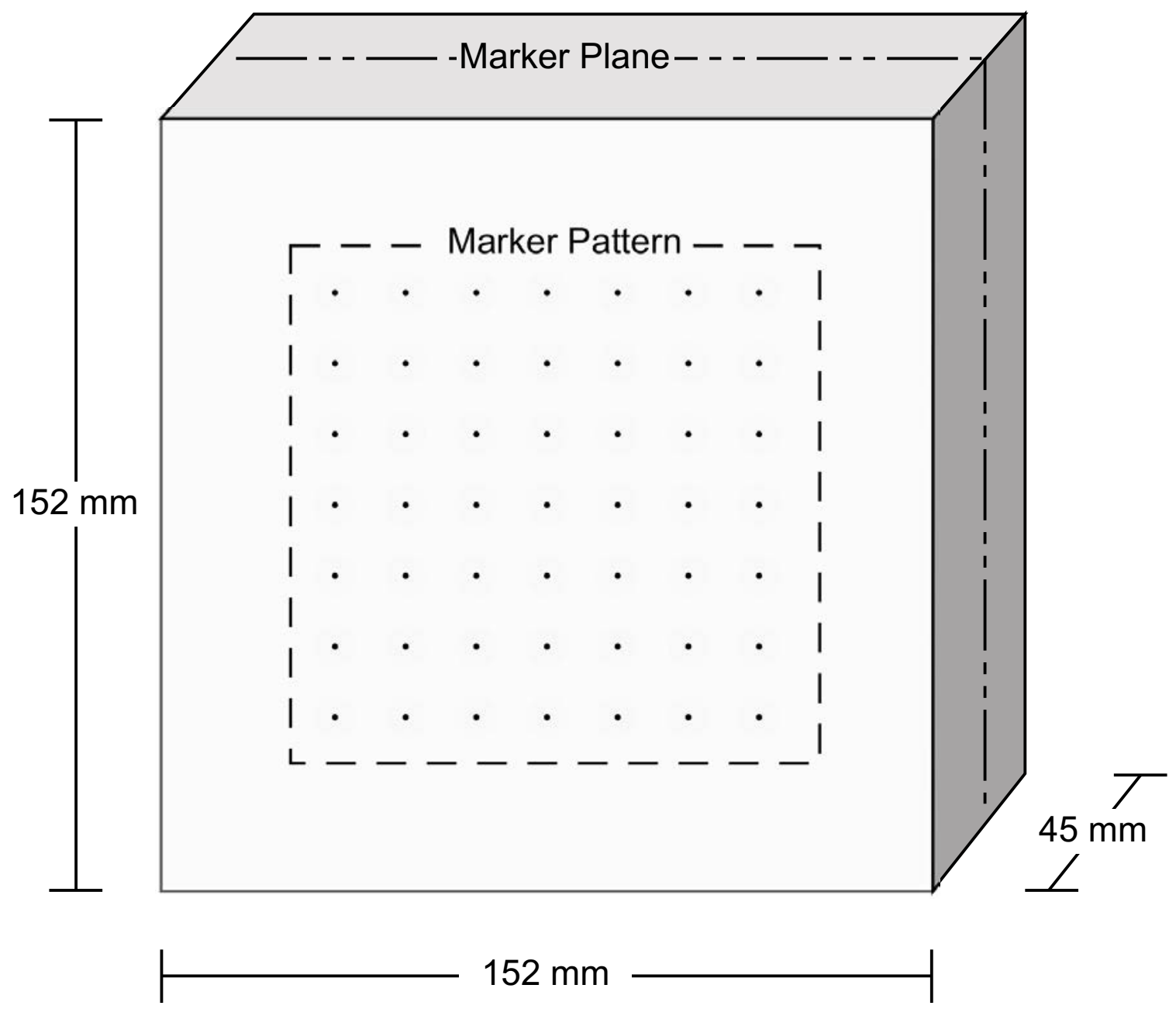

Figure 17: Dimensioned schematic of a test gel block with embedded markers. Marker patterns varied per design parameters, however they were always centred in each plane.

markers did not have any X-ray attenuating properties and were monitored optically with a high-speed camera. The NDTs were prepared based on the description of the markers provided by Hardy et al. [25], which involved embedding tin granules into $2 \mathrm{~mm}$ diameter by $5 \mathrm{~mm}$ long tubes sealed with cyanoacrylate. The lead markers were No. 6 lead shot pellets (2.8 mm diameter) and were selected to replicate markers used in earlier experiments [33]. 


\subsubsection{Marker Preparation}

The nine unique new markers were blends of a powdered contrast agent and a variety of elastomeric binders, which were used to achieve the desired material properties. All markers were cast using the same precision machined aluminum mould, producing a sheet of elastomeric radiopaque material, from which $2 \mathrm{~mm}$ diameter by $2 \mathrm{~mm}$ long cylindrical markers protruded. Marker size and shape was chosen to provide clarity in imaging and ease of manufacturing and manipulation. Barium sulfate $\left(\mathrm{BaSO}_{4}\right)$ was used as the contrast agent, as it is readily available in powdered form and suspends well into binder materials. At the X-ray power levels used in diagnostic imaging $(25-150 \mathrm{keV}), \mathrm{BaSO}_{4}$ has a high mass attenuation coefficient $\left(10<\mu / \rho<100 \mathrm{~cm}^{2} / \mathrm{g}\right)$ for a material of its density $\left(4.5 \mathrm{~g} / \mathrm{cm}^{3}\right)$, and as such is commonly used in diagnostic X-ray imaging [47]. Three different elastomeric binders were used to develop high, medium, and low stiffness markers.

The stiffest binder material was a 1:1 blend of Sylgard 184 and 527 (Dow Inc., $E=830 \mathrm{kPa}$ ), the intermediate stiffness binder was a 1:5 blend of Sylgard 184 and $527(E=130 \mathrm{kPa})$ [55], and least stiff binder was the same thermoplastic gel used in the tissue-simulant blocks (Gel \#4, Humimic Medical LLC., $E<5 \mathrm{kPa}$ ). Once placed in the mould, uncured marker preparations were degassed at $-29 \mathrm{mmHg}$ to remove small air bubbles. Markers with Sylgard-based binders were cured at room temperature, while the thermoplastic gel markers were brought to their melt temperature during casting and allowed to cool at room temperature. Variations in density were achieved by adjusting the mass ratio of elastomer binder to contrast agent. Given the random orientation of the $\mathrm{BaSO}_{4}$ particles in the elastomer matrices, the elastic moduli of the resulting composites were calculated with the Reuss mixture model [56]. The mixture model indicates 
an average increase in moduli of $30 \%$ for the composite material. Despite this increase, stiffness design parameter steps remained valid as they increased 10 fold over each other, maintaining a wide parameter space. A summary of the marker designs, their mechanical properties, and preparation variations are provided in Table 1.

Table 1: Taguchi L-9 array of marker designs

\begin{tabular}{c||ccc} 
Experiment & \multicolumn{3}{|c}{ Level } \\
& Density $\left(\mathrm{g} / \mathrm{cm}^{3}\right)$ & Spacing $(\mathrm{cm})$ & Stiffness $(\mathrm{kPa})$ \\
\hline 1 & 1.05 & 1.0 & 6 \\
2 & 1.05 & 1.5 & 170 \\
3 & 1.05 & 2.0 & 1100 \\
4 & 1.45 & 1.0 & 170 \\
5 & 1.45 & 1.5 & 1100 \\
6 & 1.45 & 2.0 & 6 \\
7 & 1.85 & 1.0 & 1100 \\
8 & 1.85 & 1.5 & 6 \\
9 & 1.85 & 2.0 & 170
\end{tabular}

To produce the thermoplastic gel test blocks, two identical reusable square moulds prepared from a 1:1 blend of Sylgard 184 and 527 were cast using a mould constructed of medium density fibreboard (MDF). These moulds were preferred for sample preparation due to the heat resistance of the thermoset material. For each block, new tissue-simulating gel was melted completely by heating to $160^{\circ} \mathrm{C}$. The liquid gel was degassed in a vacuum oven to $-29 \mathrm{mmHg}$ to remove all air bubbles, then poured into moulds, stopping at the half-way mark in order to create the marker plane. Once the half-filled blocks were set, a printed grid pattern, corresponding to the spacing level of interest for each design, was transferred to the marker plane with ink. Markers were placed on top of the pattern. Two sets of 


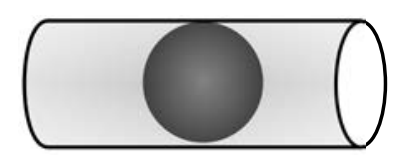

(a)

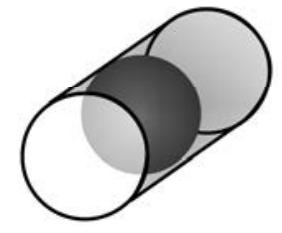

(b)

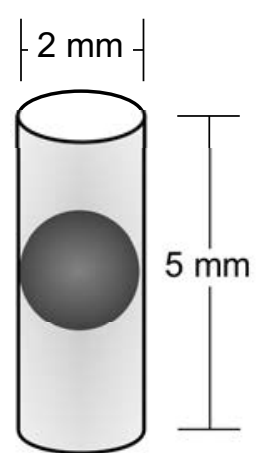

(c)

Figure 18: Orientation of NDT markers relative to marker plane. (a) Horizontal. (b) Transverse. (c) Vertical, with typical dimensions.

NDT markers were prepared for the study in order to evaluate sensitivity of cylindrical axis orientation for this rigid marker due to its notable aspect ratio. One set of NDTs were laid with the cylinder axes parallel to the marker plane, allowing investigation of vertical and horizontal orientation by rotating the entire block $90^{\circ}$. The other NDT set was laid with the axes pointing upward to investigate the transverse orientation (see Figure 18). The massless markers were created by drawing approximately $2.5 \mathrm{~mm}$ diameter circles with an alcohol-based permanent marker, at a pattern spacing of $10 \mathrm{~mm}$. To avoid deformation of the marker plane due to material melting or creep during the casting of the second half of the encasing thermoplastic block, this preparation was placed in a freezer at a temperature of $-18^{\circ} \mathrm{C}$ for a minimum of 30 minutes before the remainder of the mould was filled. Completed blocks were left to set at room temperature and inspected for seams, voids, or material inhomogeneity due to the casting process. Following inspection, each specimen was wrapped in plastic and shelved to avoid indentation, creep, or compression. The Sylgard moulds were kept and later used to house each block within a drop apparatus for impact testing. 


\subsubsection{Drop Testing}

To evaluate the dynamic response of the markers within the gel block, each specimen was mounted in a custom housing which was subsequently subjected to three drops onto a rigid steel anvil. A small custom drop tower was constructed for this purpose (see Figure 19). The custom housing was used to protect against boundary damage in order to facilitate multiple tests. Each block was inserted back into one of the two identical Sylgard moulds (encasements) used in the casting process. This assembly was then sandwiched between two $6.4 \mathrm{~mm}$-thick acrylic plates and fastened at the corners with bolts (Figure 20). The clamping pressure was controlled by restricting the distance between the sheets to $41 \mathrm{~mm}$ for all samples, ensuring that each would be subject to the same boundary conditions. The Sylgard housing reduced the tendency for the blocks to buckle out of plane or slide during impact.

The drop carriage on the drop tower was hoisted manually and fixed in place by an electromechanical latch at the maximum achievable system height of $76.5 \mathrm{~cm}$. Following each drop, the samples and housing were inspected for damage and reset to the drop position. An electronic triggering system was used to coordinate the drop with the image capture system, allowing the recording of block motion from $50 \mathrm{~ms}$ before and $130 \mathrm{~ms}$ after impact.

\subsubsection{Data Collection}

Images of the impacts were acquired using the cineradiography system described in Chapter 3 at a capture rate of $7500 \mathrm{fps}$. The resultant scale between camera and experiment was approximately $3.4 \pm 0.17 \mathrm{px} / \mathrm{mm}$. The ink-based massless marker block was tested using the same drop procedure, however as its markers do not 


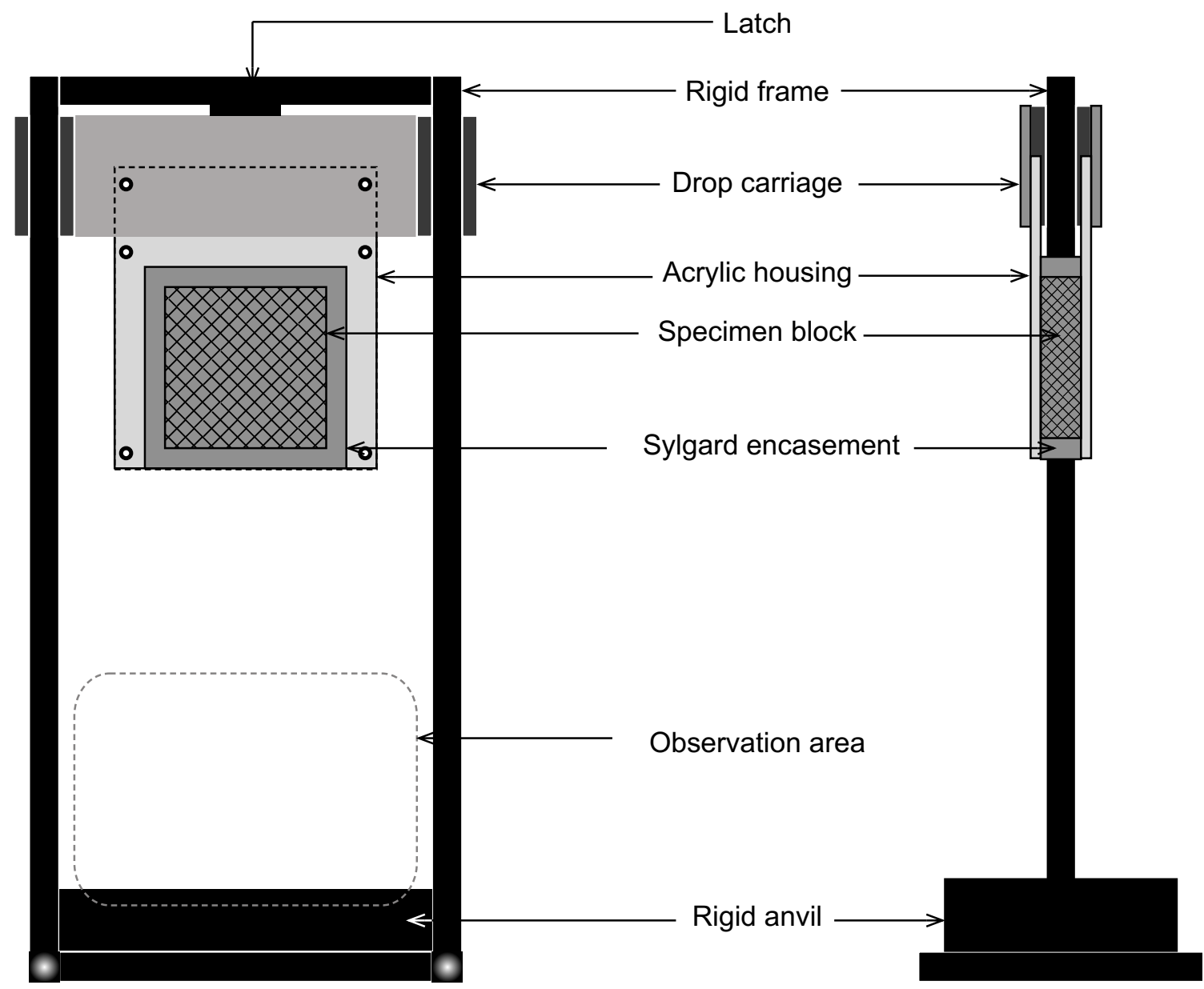

Figure 19: Front and right-section schematic views of the drop tower used in the study. Impact observation zone is denoted with a dashed line. Maximum drop height: $76.5 \mathrm{~cm}$. 

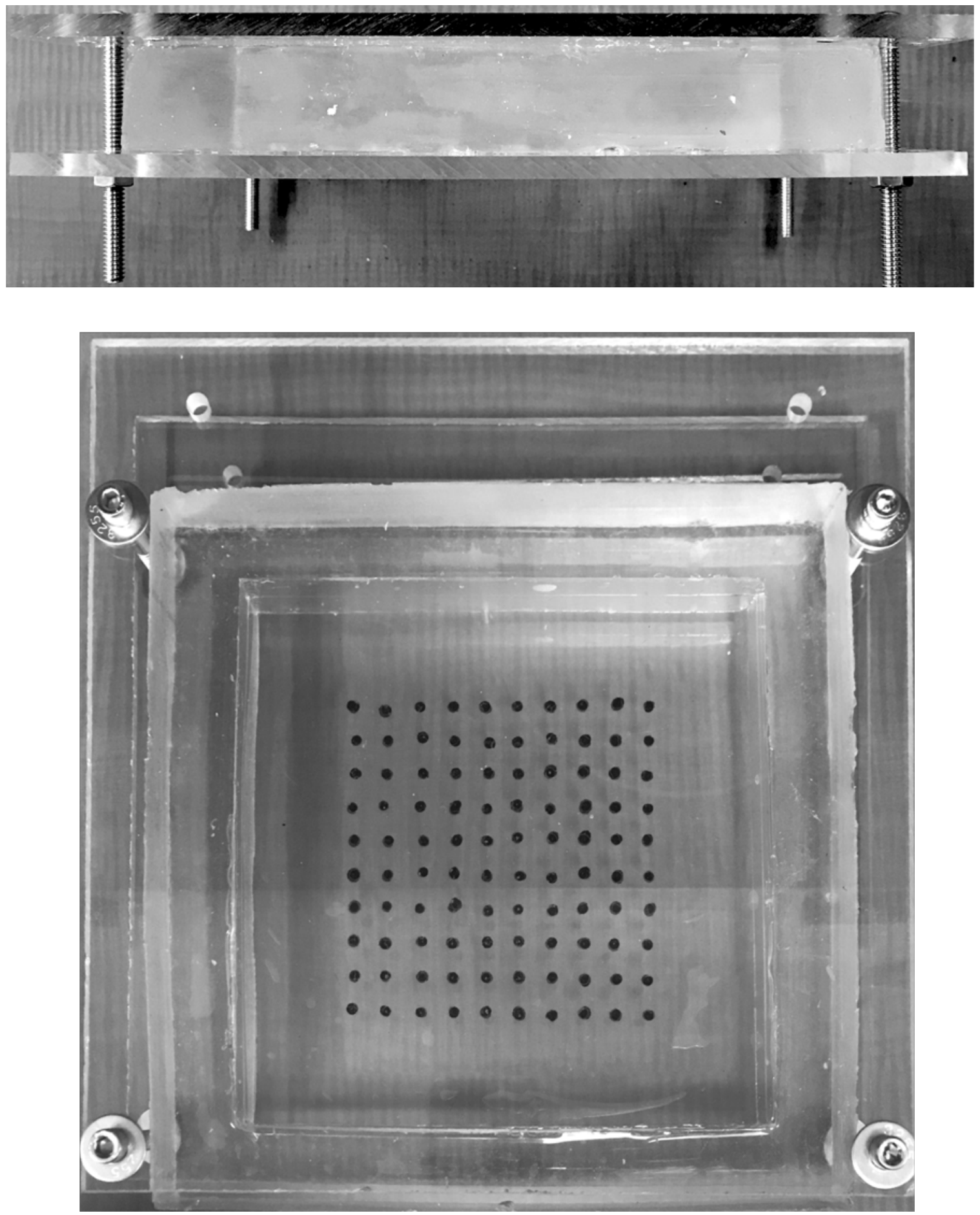

Figure 20: Front and top photographic views of the massless marker block mounted inside the drop housing. The specimen block is inserted into a Sylgard encasement and sandwiched between plates of acrylic. 
attenuate X-rays, particle motion was acquired using a high-speed optical camera at $7500 \mathrm{fps}$. Refractive and reflective distortion of the optical signal through the gel was mitigated with the use of the acrylic housing and is assumed to have a negligible effect on the observed motion of markers. Photographs and respective high-speed images of massless, NDT, and experimental markers are shown in Figure 21. Although air bubbles can be seen in the photograph of specimen \#5 (Figure 21), they were not detectable in the HSXR and were only present at the outermost boundary, not within the marker plane of the block. The presence of such bubbles is not believed to have any influence on the impact response. Image stacks containing the moment of impact and response for each drop experiment were post-processed in MATLAB [48].

In the present study, the stabilization method as described in Chapter 3 could not be used in order to fix the frame of reference to the exterior specimen housing. At the time of testing, the fiducial markers used in the stabilization process had not yet been developed. Instead, portions of the housing were observed across the time series and used to determine both the moment of impact and the rigid body motion of the housing following impact. This examination was performed by comparing a single, central column of pixels from each drop test across 250 frames $(\approx 33 \mathrm{~ms}$ ), and observing the step-wise motion of the rigid housing features. This method is outlined in Figure 22, For the time period of interest, the rebounding motion of the housing after impact was approximately linear, exhibiting a rate of $0.11 \pm 0.01 \mathrm{~mm} /$ frame. Although deformations under investigation occur within moving frames of reference, rigid body motion was equivalent across all test iterations, leaving the results across the the different specimens appropriately comparable. Following the evaluation of the moment of impact, the images were subsequently used for the particle tracking process in order to determine the 

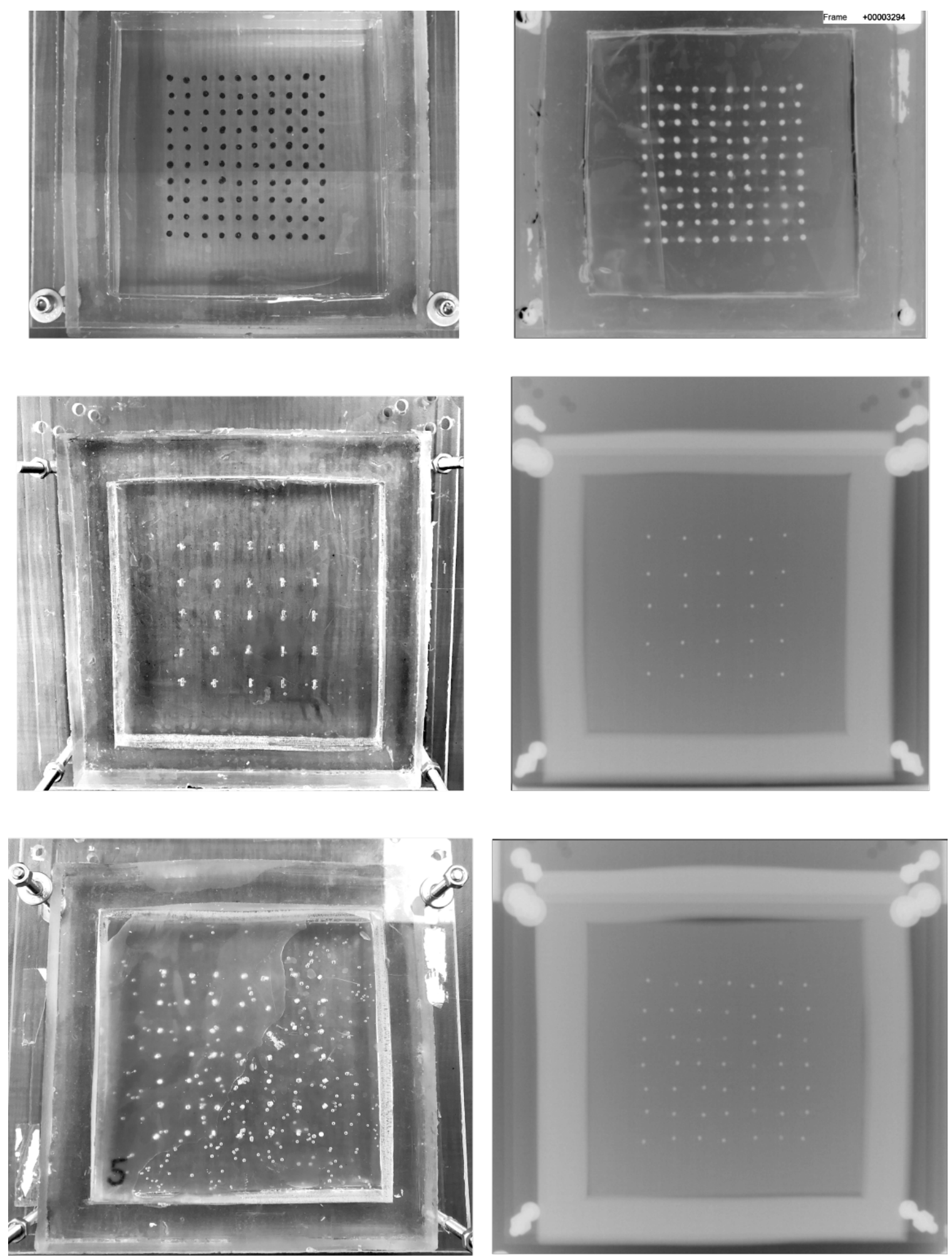

Figure 21: Photographs of specimens mounted within housing (left) and experimental image capture (right).From top to bottom: Massless, NDT, and marker design \#5. Experimental images captured optically for massless and with radiography for all others. Images adjusted for clarity. 

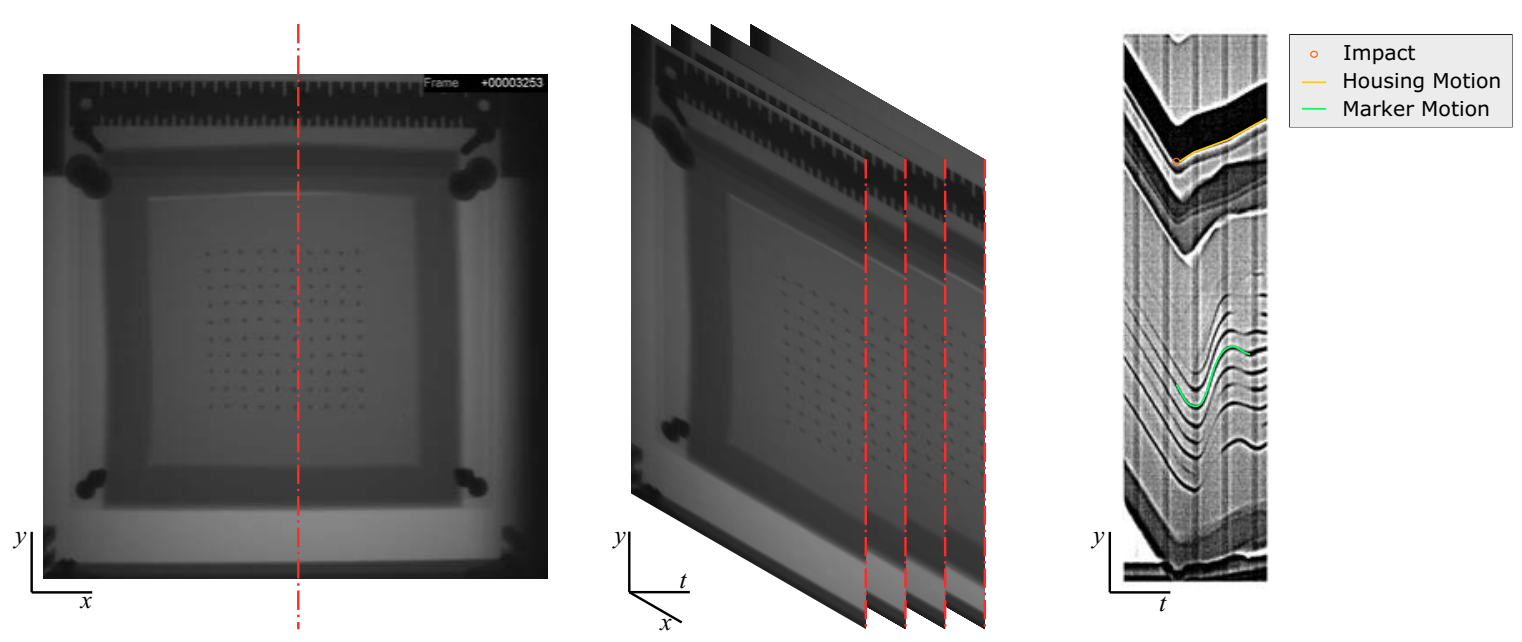

Figure 22: In order to determine the moment of impact and post-impact rigid body motion of each specimen, image stacks were analysed. Left: A central column of pixels was selected, acting as a section line. Middle: The same column of pixels was selected through the entire time-series. Right: The group of pixels was assembled, and observed in the $y$-t plane, equivalent to looking at the cut-surface of a stack of images. The moment of impact, rigid body motion, and particle motion are highlighted.

internal deformation of the specimens.

The response of each experimental marker block was compared with the massless marker configuration, which provided the drop response of the neat gel material. Within each block, markers were indexed from left to right and then top to bottom. The vertical and horizontal displacement for each marker was computed for the entire time series of approximately $75 \mathrm{~ms}$, providing motion paths for each marker. Marker motion was observed to be more erratic and less organized near the boundary. To limit boundary effects in the analysis, only markers falling within the central region (circular, $1.5 \mathrm{~cm}$ radius) in each block were chosen for analysis. The marker displacements observed over three repeated drop tests were averaged to provide a single representative curve for each marker design. Although markers were tracked in both vertical and horizontal directions, the most significant and repeatable displacements were seen in the vertical direction, given the 1-D nature of 
the drop event. All specimens were tested in the same manner, with each specimen displaying a high degree of repeatability. The mean for each design is accompanied by a $95 \%$ confidence interval (CI), which was computed with a two-tailed t-test to identify the quality of the response. The small range of the confidence interval suggests that for future drop-tests with these designs, the expected mean response will fall within a narrow region, verifying the repeatability of the observed response. This parameter should not be conflated with tolerance interval, as it represents the probability of future tests, and not the spread of the collected data. Figure 23 highlights how the drop response data was extracted and combined to create the mean and CI.

As seen in Figure 23, only minor variations in the motion profile occur within the first $15 \mathrm{~ms}$, providing a clear picture of the impact response. The cohesion of the marker motion tended to dissipate into the second period $(t>25 \mathrm{~ms})$, reducing repeatability at later times. In previous studies [24, 25], peak displacements observed shortly after impact have been used as a basis of measurement for tissue deformation and characterization. For this study, the first and second peaks of the first period response were used as the basis of comparison across all specimens, as the data was both cohesive and relevant to tissue-based impact studies. The first peak represents the compressive phase following impact, and the second peak represents the relaxation and release of stored elastic energy following compression.

The normalized output values of this analysis $(\phi)$ were defined as

$$
\phi=\frac{\chi_{t}-\chi_{n}}{\chi_{n}} \times 100,
$$

where $\chi_{n}$ and $\chi_{t}$ are the measured values of a given response parameter for the neat material and the material containing a test marker, respectively. The output 
1
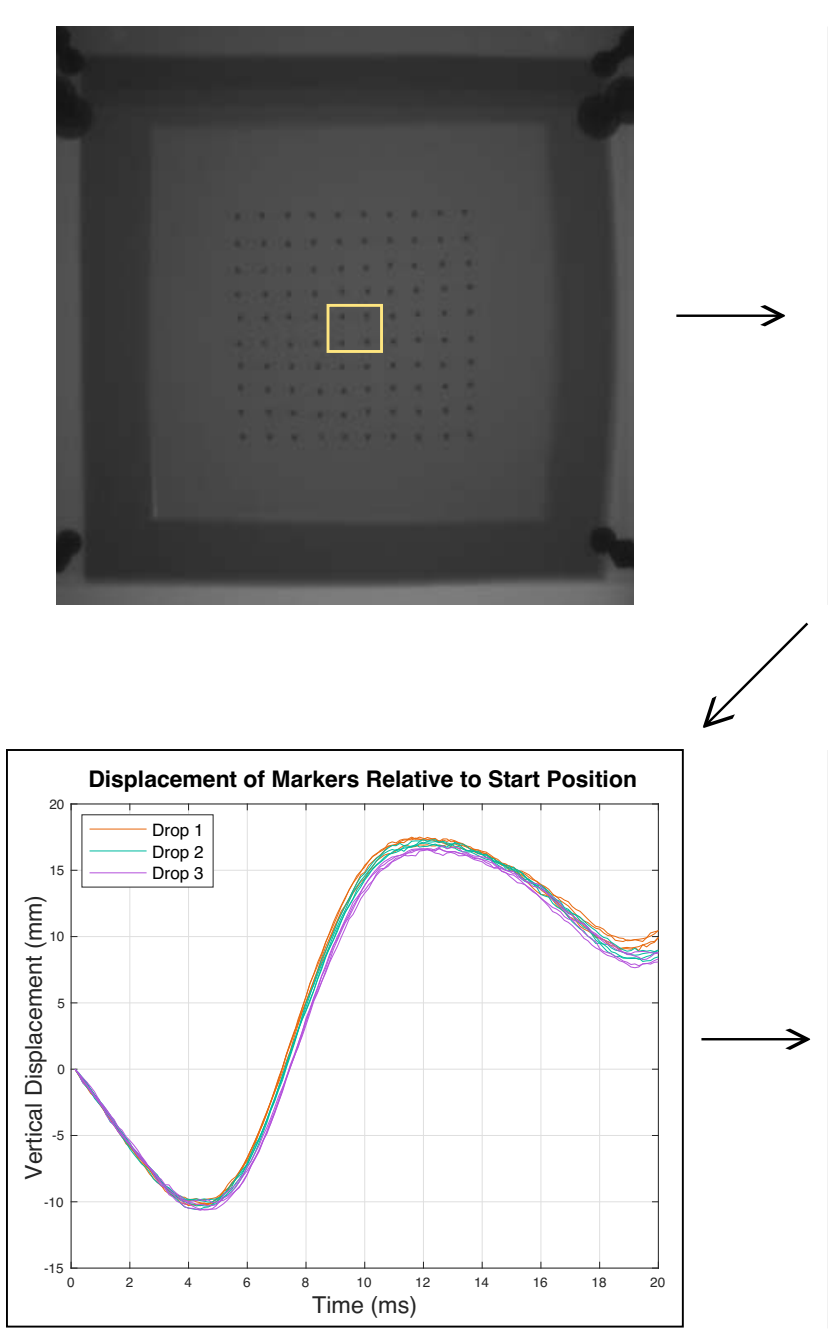

3
2
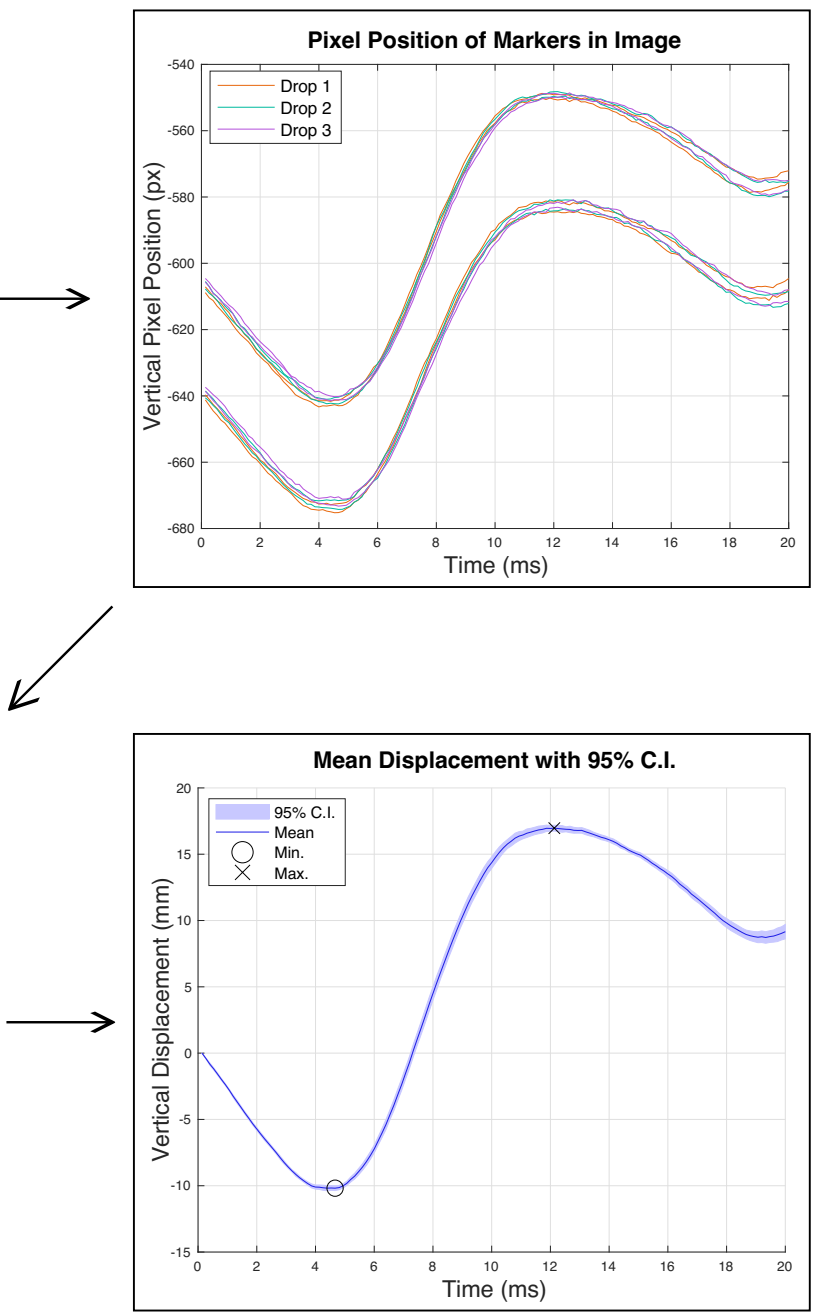

4

Figure 23: The curve used for analysis of marker Design 7 was extracted by selection of central markers (1) for pixel position tracking. The pixel positions (2), were converted from pixels to millimetres and zeroed relative to their moment-of-impact positions, creating a displacement curve (3). The displacement curves were averaged to create the mean displacement and CI curve (4), where the peak first and second displacements were extracted. The moment of impact occurs at $t=0 \mathrm{~ms}$, with the first peak occurring at $t \approx 4 \mathrm{~ms}$ and the second peak occurring at $t \approx 13 \mathrm{~ms}$. 
value provides a measure of deviation, as caused by the presence of a marker particle, from the neat material response. These output values $(\phi)$ were subsequently used in the Taguchi signal-to-noise function to make balanced comparisons across the experimental group. From this data, the sensitivity of the response to each of the marker design parameters could be evaluated.

\subsubsection{Analysis}

The Taguchi approach to experimentation was used to reduce the number of specimen iterations required to determine parameter sensitivity, which is a notable shift away from the traditional full factorial experimental method of testing every iteration [54 [57]. In the full factorial method, one would perform a separate experiment for each change of every variable, increasing the number of required experiments exponentially as the number of variables increases. The Taguchi orthogonal array provides a balanced method of combining variable adjustments across individual experimental trials, as shown in the marker design parameters in Table 1 . This method has seen broad uptake in many fields, including the biomedical and clinical research fields to reduce the burden of experimental cost and supply limitations [58]. The technique is of particular benefit to the medical field as the ability to develop robust results despite the contribution of external noise factors, such as patient histories and anatomical variations.

In order to separate the valuable data from the noise influence, this method uses signal-to-noise ratios $(\mathrm{SNr})$ as a measure of experimental output due to varying design parameters. $\mathrm{SNr}$ calculations can focus on a nominal value, a smallestis-best, or a largest-is-best output. In the case of this study, the highest-is-best evaluation was used, as per: 


$$
S N r=-10 \log _{10}\left[\frac{1}{n}\left(\sum_{i=1}^{n} \phi_{i}^{2}\right)\right],
$$

where $\phi$ is the measured output for each experiment, $S N r$ is the signal-to-noise ratio, and $n$ is the number of repeated trials.

The $S N r$ for each experiment is used to evaluate the design variable influence for each of the output responses. The mean $S N r$ for each parameter at each level is computed as

$$
\overline{S N r_{P L}}=\frac{1}{k} \sum S N r_{i}
$$

where $L$ is the parameter level, $P$ represents the specific parameter, and $k$ is the number of trials in which that combination appears. The index, $i$, corresponds to the specific trials containing the $P L$ combination. For example, the mean $S N r$ for the stiffness parameter at its lowest level would be the mean for experiments 1,6, and 8 , as can be seen when consulting Table 1

While the $S N r$ means can be used to determine how each design parameter affects the output measurement, they cannot attribute the magnitude of the effect from each parameter. To achieve this, an analysis of variance (ANOVA) is required [54,59]. ANOVA relates the contribution of the variance for each design parameter to the total experimental variance. The total variance $\left(S S_{t}\right)$ for each experiment is equal to the sum of the design parameter variances $\left(\sum_{i=1}^{n} S S_{i}\right),(n=3)$ and the external variance, or noise $\left(S S_{e}\right)$ [60]. Whereas the $S N r$ ratio provides information on which design parameter levels may be desirable, the magnitude provides information on the overall sensitivity of that parameter.

$$
S S_{t}=S S_{e}+\sum_{i=1}^{n} S S_{i}
$$




$$
S S_{P}=\sum_{j=1}^{k}\left(\frac{\left(\sum S N r_{L}\right)^{2}}{k}\right)-\frac{G^{2}}{n},
$$

where $G$ is the sum of all of the results from each of the $n$ experiments and $k$ is the number of experiments of each design parameter $P$ at each level, $L$.

\subsection{Results}

The resultant first and second internal peak displacements for blocks embedded with the new marker designs are used to identify how the individual design parameters (density, stiffness, and spacing) contribute to the deviation in the response as compared to the displacements measured in the neat material. The same internal displacements measured in blocks containing NDT and lead markers are also compared to the neat material to evaluate their overall performance and not the influence of their parameters. As such, only the new marker designs are used in the Taguchi analysis, as their designs fall within the appropriate design space.

\subsubsection{New Marker Designs}

The first and second peak displacements for each of the new marker designs are seen in Table 2 along with the associated range of the $95 \%$ confidence interval, and Taguchi $S N r$ ratio, as per equations (4) and (5). The $S N r$ value functions as a highest-is-best representation for each response, and appropriately scales external noise factors. The values used are the mean vertical displacements of material due to impact. Each response was generated from a number of input displacement traces, belonging to 3 to 12 markers depending on the grid spacing used in the design. Each set of markers was chosen to represent the of the block under observation. The centre region was chosen as to lessen the effect of erratic motion 
observed at the boundary. The vertical motion profiles of these markers are shown together with the neat material profile in Figure 24.

Table 2: Experimental outcomes from drop test and respective $S N r$.

\begin{tabular}{|c|c|c|c|c|c|c|}
\hline \multirow[t]{2}{*}{ Experiment } & \multicolumn{3}{|c|}{ First Peak } & \multicolumn{3}{|c|}{ Second Peak } \\
\hline & $(m m)$ & C.I. $( \pm m m)$ & $\operatorname{SNr}(d B)$ & $(m m)$ & C.I. $( \pm m m)$ & $\operatorname{SNr}(d B)$ \\
\hline Neat & 11.8 & 0.2 & - & 18.6 & 0.9 & - \\
\hline 1 & 10.6 & 0.3 & 19.6 & 20.3 & 0.4 & 21.1 \\
\hline 2 & 11.5 & 0.4 & 31.1 & 16.9 & 0.9 & 20.6 \\
\hline 3 & 8.1 & 0.4 & 10.0 & 22.8 & 0.8 & 13.0 \\
\hline 4 & 12.2 & 0.3 & 30.1 & 18.7 & 0.6 & 45.5 \\
\hline 5 & 12.2 & 0.3 & 29.0 & 10.5 & 0.5 & 7.2 \\
\hline 6 & 11.2 & 0.1 & 25.4 & 20.7 & 1.0 & 19.3 \\
\hline 7 & 10.2 & 0.2 & 17.3 & 17.0 & 0.2 & 20.9 \\
\hline 8 & 9.6 & 0.2 & 14.8 & 17.9 & 0.3 & 27.7 \\
\hline 9 & 10.3 & 0.2 & 18.0 & 20.4 & 0.4 & 20.7 \\
\hline
\end{tabular}

The influence of design variables on the deviation from neat material response can also be shown by examining the mean $S N r$ for each variable at each of its different intensity levels (L1, L2, or L3), as per equation 6. This method considers responses, not just the best or worst performers. In this evaluation, only the new marker designs were considered, as NDT and lead marker parameters fall outside 


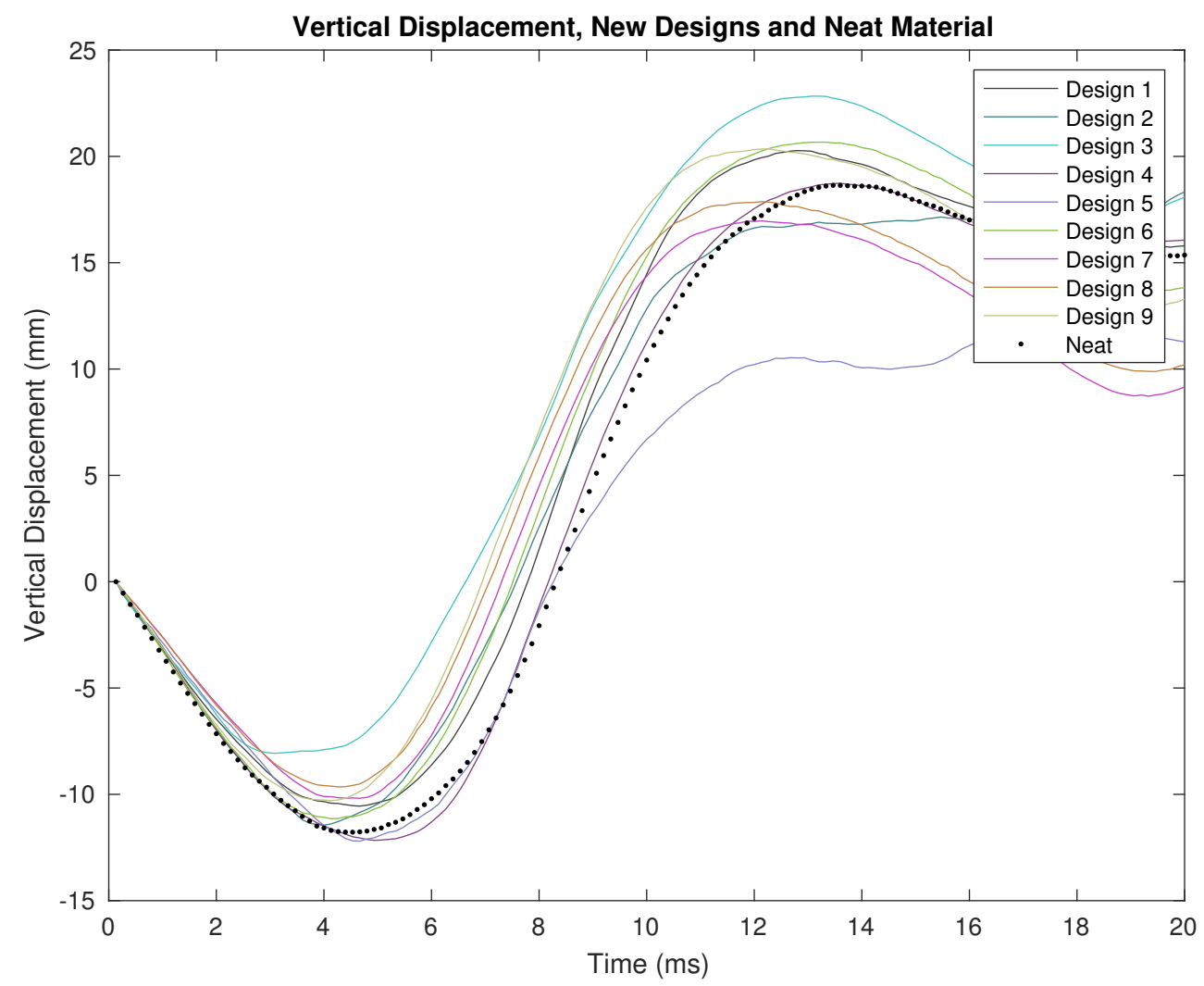

Figure 24: Mean vertical response of new marker designs, as compared to the response of neat material. Curves are aligned at the moment of impact. 
of the Taguchi design space. For each design variable, the spread of the means of $\mathrm{SNr} r$ values highlights how sensitive the output metric is to changes in the variable intensity (Table 1). These results are shown in Tables 3 and 4 In Table 3 , the greatest range is associated with density, while in Table 4 , the greatest spread is associated with stiffness. These results echo what was seen from the top performers from Table 2. While comparison of the mean $\mathrm{SNr}$ provides insight into how each design variable contributes to the deviation of the output, it does not evaluate the strength of the contribution.

Table 3: Mean $S N r$ at Peak 1

\begin{tabular}{|c|c|c|c|c|}
\hline \multirow[b]{2}{*}{ Parameter } & \multicolumn{3}{|c|}{ Mean $S N r$} & \multirow[t]{2}{*}{ Spread } \\
\hline & L1 & L2 & L3 & \\
\hline Density & 20.2 & 28.2 & 16.7 & 11.4 \\
\hline Spacing & 22.3 & 24.9 & 17.8 & 7.1 \\
\hline Stiffness & 20.0 & 26.4 & 18.8 & 7.6 \\
\hline
\end{tabular}

Table 4: Mean SNr at Peak 2

\begin{tabular}{|c|c|c|c|c|}
\hline \multirow[b]{2}{*}{ Parameter } & \multicolumn{3}{|c|}{ Mean $S N r$} & \multirow[t]{2}{*}{ Spread } \\
\hline & L1 & L2 & L3 & \\
\hline Density & 18.2 & 24.0 & 23.1 & 5.7 \\
\hline Spacing & 29.2 & 18.5 & 17.6 & 11.5 \\
\hline Stiffness & 22.7 & 28.9 & 13.7 & 15.2 \\
\hline
\end{tabular}

Using the ANOVA described in equations (7) and (8), the sensitivity of both the input design variables and the external noise are evaluated. These results are shown in Table 5 as representative percentages of the total sensitivity. As expected, 
at the first peak, density holds the greatest influence over the output, while stiffness and spacing contribute similarly to each other. External noise also has a contribution, which is contributed by unknown or uncontrollable factors. At the second peak, the dominating factor is stiffness, and external noise is increased, suggesting greater parameter interaction and stochastic behaviour as time progresses.

Table 5: Parameter sensitivity (\%)from ANOVA

\begin{tabular}{c||cc} 
Parameter & Peak 1 & Peak 2 \\
\hline Density & 46 & 6 \\
Spacing & 18 & 28 \\
Stiffness & 22 & 39 \\
External Noise & 14 & 27
\end{tabular}

\subsubsection{NDT, Lead Markers}

The vertical motion profile of the markers used in past studies (NDT, lead) is shown together with that of the neat material in Figure 25. The first and second peak displacements and corresponding confidence intervals and Taguchi $S N r$ values are shown for these markers in Table 6. NDT orientations are denoted by " $\mathrm{H}$ " for horizontal, " $\mathrm{T}$ " for transverse, and " $\mathrm{V}$ " for vertical. These results show a wide range of deviation as compared to the response of the neat material. The use of these markers for effectively determining the displacement of the neat gel material does not appear to provide any particular advantage over that of the newly designed markers. 


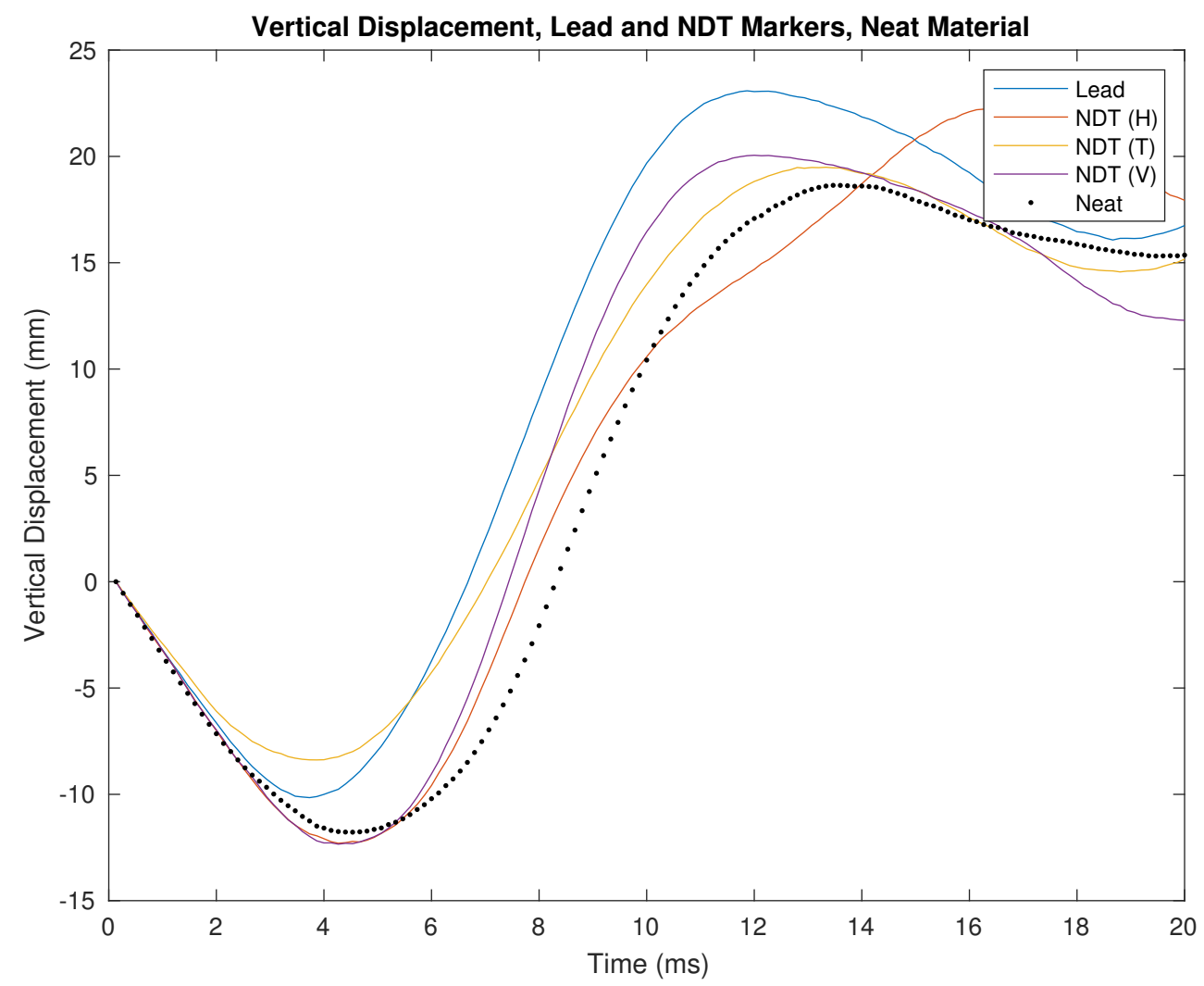

Figure 25: Mean vertical response of previously used markers, as compared to the response of neat material. Curves are aligned at the moment of impact. 
Table 6: Experimental outcomes from drop test and respective $S N r$.

\begin{tabular}{c||cccccc}
\multicolumn{1}{c||}{ Experiment } & \multicolumn{3}{c}{ First Peak } & \multicolumn{3}{c}{ Second Peak } \\
& $(m m)$ & C.I. $( \pm m m)$ & $\mathrm{SNr}(d B)$ & $(m m)$ & C.I. $( \pm m m)$ & $\mathrm{SNr}(d B)$ \\
\hline Lead & 10.2 & 0.15 & 17.2 & 23.1 & 0.5 & 12.5 \\
NDT - H & 12.3 & 0.15 & 27.1 & 22.3 & 0.8 & 14.1 \\
NDT - T & 8.4 & 0.12 & 10.8 & 19.5 & 0.2 & 26.8 \\
NDT - V & 12.3 & 0.17 & 26.5 & 20.1 & 0.2 & 22.4
\end{tabular}

\subsubsection{Discussion}

In comparing the displacement response of the neat gel block with those blocks containing new marker designs, it can be seen that there is a broad variability in the magnitude of the displacements at the first and second peak in the vertical motion profile. Using the greatest-is-best Taguchi $S N r$, the best performer early in the response (first peak) is Design 2, as expected, as its density is similar to that of the neat material, while its spacing and stiffness are in the middle of the design space. Designs 4, 5, and 6 also perform well at the first peak, which is likely a function of their medium density, which is the driving factor at this peak. The second peak responses differ more substantially, which is expected as the influence from external factors increases by two-fold at this stage. Here, the best performer is Design 4, while Designs 2, 5, and 6 fall to the middle of the group.

Comparing the first-peak results with respect to marker design properties, medium densities are the best performers, while it appears that low stiffness markers tend to perform better than those with a high stiffness. Markers in tight and medium spacing patterns approximate the neat material response more closely. At the second peak, where noise is increased, density does not appear to have much of an effect, while lower stiffness approximates the neat material well. Medium and high stiffness markers have opposing responses, suggesting some modal stiffness 
effects. Unlike the first peak response, broad spacing tends to cause the markers to undershoot at the second peak, while tight and medium spacing approximate the true response more accurately. These spacing effects also suggest modal interactions due to the oscillatory nature of the response.

The sensitivity information provided by the ANOVA shows how each of the design parameters influence the response, relative to each other and the external noise factors. These sensitivity values reinforce what was learned from the $\mathrm{SNr}$ comparisons, suggesting that early on, marker density (46\%) plays a significant role in the tuning of markers to a particular material. Stiffness (22\%) and spacing $(18 \%)$ play similar roles, and are greater than that of the external noise $(14 \%)$, and as such must be considered in marker design. At the second peak, these values shift towards higher stiffness and spacing sensitivity (39\%, 28\%, respectively), while density falls substantially $(6 \%)$. With the increased external noise $(27 \%)$, response parameters at this peak are less reproducible and contain a greater degree of scatter. As such, the first-peak responses will provide a better guide to marker development.

Turning to the replicated markers used in previous studies, a similar spread of displacements is seen at both the first and second peak. The NDT-embedded blocks exhibited the greatest range in their ability to mimic the response of neat material. At the first peak, when NDTs were oriented horizontally and vertically, the response was similar ( $\mathrm{SNr}$ of 27.1 and 26.5 respectively), while the specimen with the NDTs transversely oriented was the second poorest performer overall. At the second peak, the transverse orientation and vertical orientation were both among the better performers, although they did overestimate the displacement seen in the neat material. What is most interesting is the effect of the orientationspecific behaviours, which are seen in the motion profiles in Figure 26 . In the horizontally oriented specimen, there is a sharp, repeatable, rate change at $13 \mathrm{~ms}$, and 

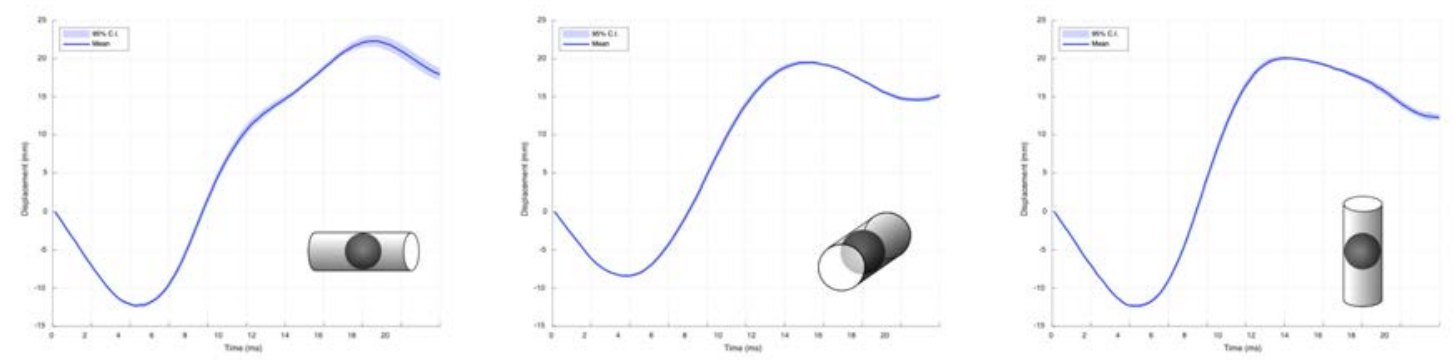

Figure 26: Full motion profiles of NDTs for each orientation. The effects of changing orientation can be seen in the sharp rate change in the horizontal orientation at $\approx 13 \mathrm{~ms}$, as well as the overall reduced amplitude in the transverse orientation.

a surprising variation in amplitude across all three orientations. The NDT markers have a high stiffness and a low average density, however their mass is concentrated at their centre, making them more similar to the lead markers than that of the new markers with low densities. This combined with their non-uniform cylindrical shape leads to a resultant displacement measurement with a high margin of error, as compared to a purely neat specimen.

The high-density, high-stiffness lead markers presented a reduced displacement at the first peak $(10.2 \mathrm{~mm})$ and an increased displacement at the second peak (23.1 mm). This combination of marker properties may reinforce the matrix material in compression, but aid in its tensile expansion due to the inertia associated with particles of a higher mass. Despite lead being far different in composition than any of the new marker designs, it was not the poorest performer, which suggests there is a counteracting interaction between density and stiffness at the first peak, which is reflected in the external noise found using ANOVA. At the second peak, the lead specimen performs similarly to other dense and stiff specimens, suggesting that at high levels, these properties may overtake the influence of spacing. 


\subsubsection{Summary}

It can be seen that Design 2 provides the best average response, while Designs 4, 5, and 6 are within a small range of each other and provide the next best response. Within this group, all properties except high density are represented, clearly indicating that carefully selected combinations of parameters, and not individual qualities, make for good candidates. From the analysis, it seems that low to medium density and stiffness make the best markers. Individually, the NDTs in horizontal and vertical orientations perform similarly, but the transverse response reduces their viability significantly. The highest density markers, including lead, consistently perform poorly.

Both image-based and ultrasound-based particle tracking systems can be used in PMHS impact studies with a high degree of accuracy precision. Although these systems are well suited to identify how tracking particles move following an impact, the particles themselves introduce their own uncertainty. From this study, it can be seen that the uncertainty on displacement measurements and subsequently calculated strain values is a function of marker design, placement, and time-afterimpact.

A clearer understanding of the error associated with the measured displacement of tissues has been developed. Moving forward to the PMHS impact study outlined in Chapter 5, consideration of these details has been given to the embedded markers used. Although Design 2 would have been the top choice, its X-Ray attenuation was too low for effective use. Ultimately, a marker similar to Design 6 was chosen. The stiffness and density of Design 6 seem to balance out its lower spacing, which is achievable in PMHS insertion. This marker was chosen above the other top performers as it shared a similar average response, but also proved to have better X-Ray attenuation, manufacturability, and ease of PMHS insertion. 


\section{Chapter 5}

\section{PMHS Experiment Details}

A key goal of this study is to observe the motion of the brain with a higher degree of clarity than previously achieved. The techniques used for image capture, processing, and measuring displacement have been described (Chapter 3) and used to evaluate the design of embedded contrast markers (Chapter 4). While these technical components are necessary for the PMHS study, they do not describe the protocol for working on the cadaveric specimens. A robust, comprehensive approach to the preparation and impacting of the PMHS heads used in this study was developed through preliminary work and trials with synthetic head forms and embalmed human specimens. Some variations to methods used in past works have been made, in efforts to improve the fidelity of specimens.

Specimens used for the present study were obtained through the Body Bequeathal Program at University of Ottawa, within the Faculty of Medicine and the Department of Innovation in Medical Education. Two specimens are discussed for the present study, numbered 2482 and 2871. Following approval from the local coroner for the release of the body to the Anatomy Laboratory, each was subject to evaluation of inclusion criteria in order to reduce the risk of performing the data collection protocol on an outlier specimen. The inclusion criteria were:

- No longer than 72 hours post-mortem at the time of release 
- Less than 80 years of age

- No known brain anomaly or neurological issue (including dementia)

- No known head or brain injuries

- Free from cancers that typically metastasize to the brain

- No freezing or embalming has been performed

Once deemed suitable for the data collection, specimens were prepared for the impact study by the prosector. Prepared specimens were then transferred to the Royal Ottawa Hospital for a pre-impact MRI, and then to Carleton for the remainder of the impact protocol. Following the impact protocol, specimens were transferred back to the Royal Ottawa Hospital for a post-impact MRI. Finally, specimens were returned to the Anatomy Laboratory where they were fixed with a formalin solution and stored for later dissection.

This chapter discusses the experimental protocol used for all specimens, both past and future. Where specific examples are required, Specimen 2871 will be used as the standard case.

\subsection{PMHS Preparation}

The prosector at University of Ottawa prepared the PMHS for the study. Heads were removed from the body between the fourth and fifth cervical vertebrae in order to maintain the integrity of the vasculature. Once separated, the third and fourth cervical vertebrae were removed, exposing the vertebral arteries and spinal cord. Further dissection of the neck was performed to expose the left and right common carotid arteries and jugular veins that were required for artificial perfusion. All tissue inferior from the base of the skull (occipital protuberance) was removed to expose the inferior surface of the occipital bone, leaving the first and 
second cervical vertebrae intact and exposed as required for mounting and instrumentation. All hair was removed from the face and scalp. Compression bandage was used to wrap the specimens to cover facial features. An illustration of the prepared specimen is seen in Figure 27.

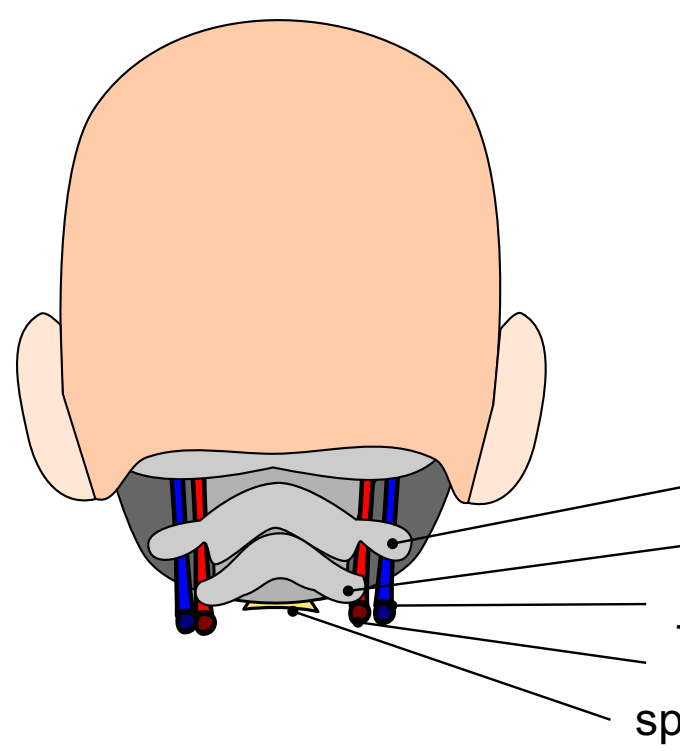

C1

$\mathrm{C} 2$

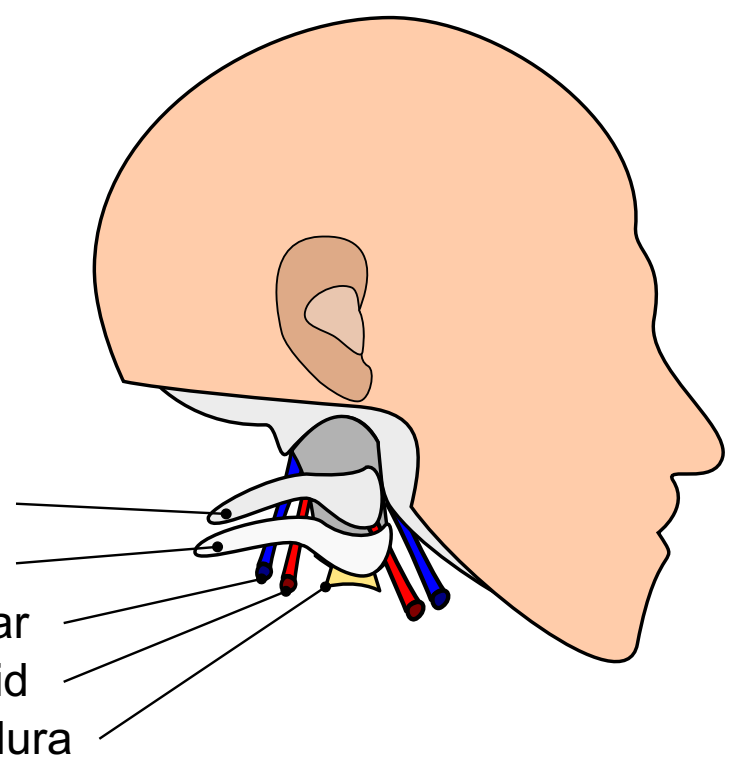

Figure 27: Illustration of specimen, as received from University of Ottawa, with spine dissected.

\subsection{Diagnostic Imaging and Evaluation}

Prepared specimens were transported to the Royal Ottawa Hospital for preliminary magnetic resonance imaging (MRI) as well as post-experiment MRI. Preliminary imaging was performed to ensure that no unexpected anomalies were present within the brain tissue, such as abscesses or tumours, and to develop a geometric understanding of each individual specimen. MRI acquisition was performed by Dr. Greg Cron and Dr. Gerd Melkus using a Siemens MRI system which is calibrated for clinical use. Although a variety of imaging protocols were tested, it was necessary to choose a protocol that would show both the structure of the brain and 
the contrast markers that would later be embedded in the brain tissue. As such, the MRI protocol chosen for this study was a T1-weighted 3D FLASH sequence with a flip angle of $60^{\circ}$. The protocol captured $1 \mathrm{~mm}$ voxels of the entire specimen, allowing for tomographic views of any plane within the specimen. A sample series of tomographic images from Specimen 2871 is shown in Figure 28, Sets of tomographic images, sliced in the sagittal, coronal, and axial planes, were generated and sent to neurosurgeons, Dr. Katherine Poon and Dr. Susan Brien, to confirm that the specimens were suitable for the study.

Upon confirmation from the neurosurgeons that a specimen was viable, it was transported to the Biomaterials Laboratory at Carleton University. Images were saved in the DICOM format and opened in OsiriX DICOM viewer [61]. Using this software, a unique target map was created for each specimen in order to target key anatomical regions in the brain for radiographic contrast marker placement, while avoiding regions that would not support marker placement (i.e., lateral ventricle). Localization of target regions was performed by first identifying external anatomical features and subsequently measuring offset distances using a Cartesian coordinate system. The target positions were superimposed on the slices of interest within the viewing software, with offset distances read aloud during the marker insertion procedure. A target map is shown in Figure 29 indicating the target regions for Specimen 2871. Target mapping was completed concurrently with the specimen mounting and instrumentation.

\subsection{Specimen Mounting and Instrumentation}

Mounting and instrumentation of the specimen was completed at Carleton University in the Biomaterials Laboratory. This process was required to create a suitable 


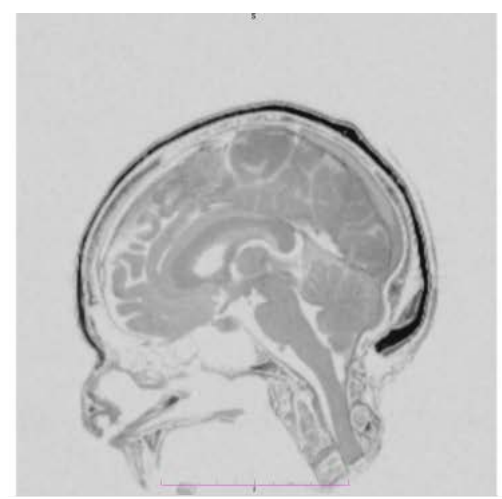

(a)

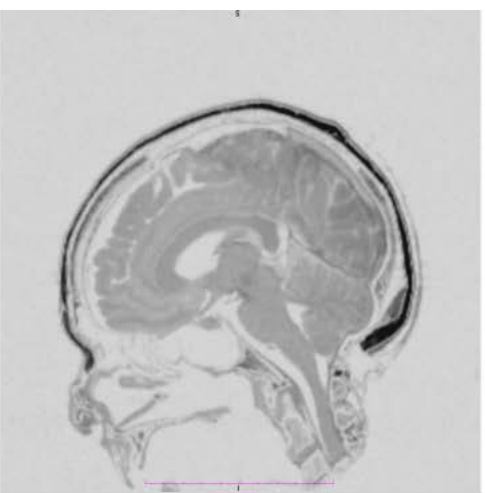

(b)

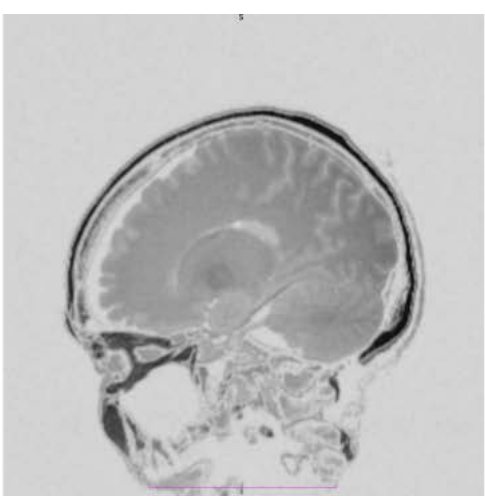

(c)

Figure 28: Tomographic images of PMHS specimen before marker insertion. (a) Target slice for brainstem markers. (b) Target slice for corpus callosum markers. (c) Target parasagittal slice for primary marker grid.

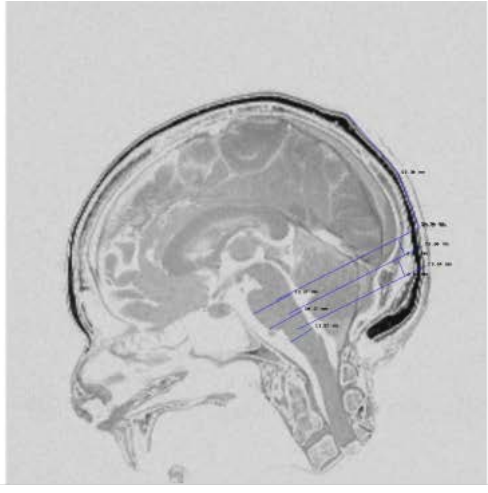

(a)

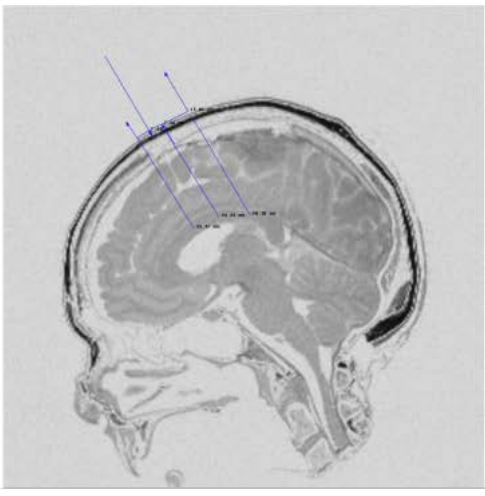

(b)

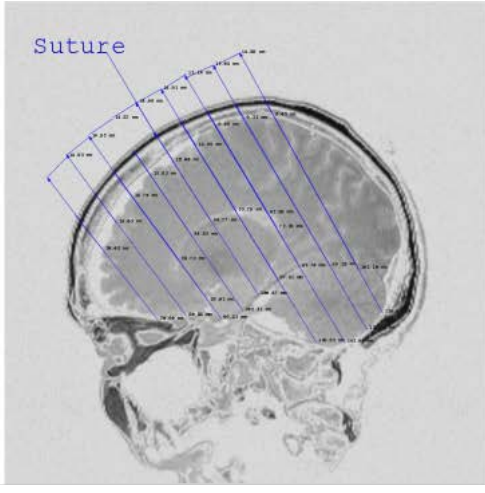

(c)

Figure 29: Overlay of marker target positions, used as a guide for the marker insertion process. (a) Brainstem. (b) Corpus callosum. (c) Parasagittal grid. 
mounting point for the specimen during the instrumentation phase of the protocol, as well as a a reliable and repeatable connection point for the unbiased neck used in the impact experiment. The unbiased neck is based on the Hybrid III neck, however it lacks a directional bias making it more suitable for head impact studies [62]. As part of this process, anthropometric measurements of the specimen, as well as its mass, were recorded to establish comparison points across the entire experimental set. The set of measurements recorded are shown in Figure 30.
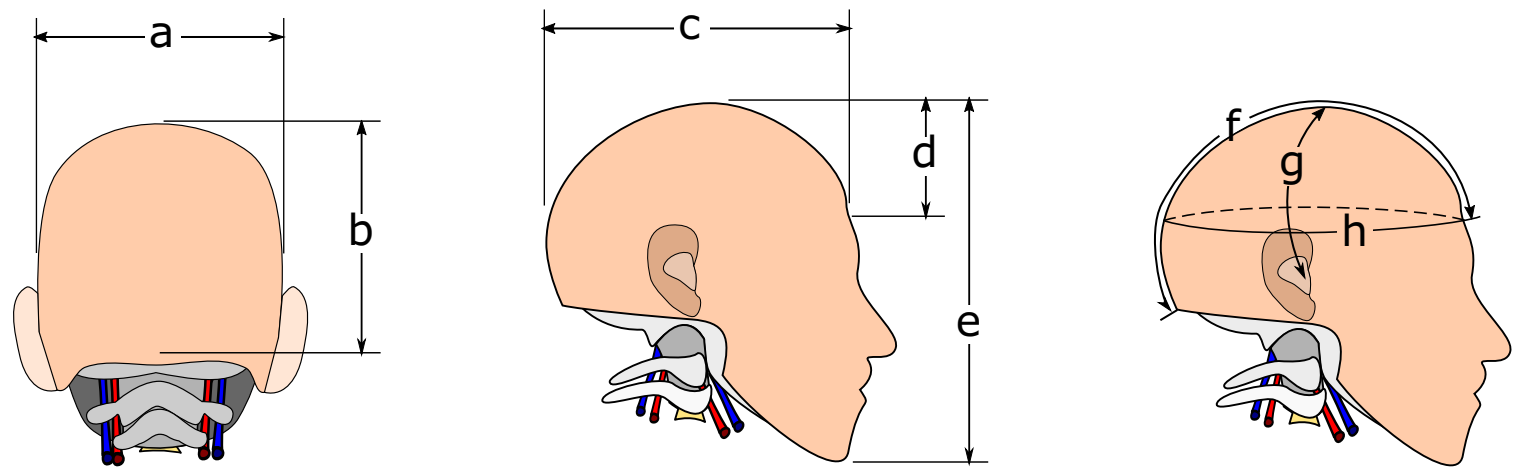

Figure 30: Anthropometric measurements recorded for each specimen, corresponding to table of measurements in Appendix D. All measurements require specimen to be parallel to Frankfort plane. a) Width at ear canal b) Superior maximum to occipital protuberance c) Nasal bone to posterior max. $d$ ) Nasal bone to superior max. e) Superior max. to inferior of mandible f) Surface length occipital protuberance to nasal bone g) Surface length ear canal to superior max. (sagittal suture) h) Circumference at nasal bone parallel to Frankfort Plane.

\subsubsection{Mounting}

The mounting point was created by applying a novel collar (Figure 31) to the base of the skull, surrounding the exposed cervical vertebrae ( $\mathrm{C} 1$ and $\mathrm{C} 2)$. The collar was cast from a 2-part urethane resin (Smooth-Cast ONYX-SLOW, SmoothON Inc.). The collar was placed on the inverted specimen and aligned such that its mounting plane was parallel to the Frankfort Plane and its angular orientation was aligned with the mid-sagittal plane (Figure 32). Modelling clay (Form-iT) was 
used to shim the collar during the alignment process. Once aligned, the collar was secured in place using up to six (6) No. $82-1 / 2$ " to 3 " long wood screws, each of which was driven into a pre-drilled $1 / 8^{\prime \prime}$ pilot hole. A depth limiting device was used to prevent pilot holes being drilled beyond the thickness of the skull. Two additional No. 81 " brass screws were inserted $1 / 4-3 / 8^{\prime \prime}$ into the remaining exposed occipital bone inside the collar, with the balance of their length left proud of the skull. These screws were positioned to act as anchor points for the potting material used to fill the collar volume. As these screws could not be retrieved following the experiment, brass was used to reduce MRI complications. Once secured, modelling clay was used to seal any gaps between the occipital bone and the collar, as well as gaps around the mandible in preparation for potting. Vasculature used for artificial perfusion, including left and right common carotid arteries, jugular veins, and vertebral arteries, were fitted with barbed fittings when possible, and routed outside of the neck collar through small, pre-cast openings in the collar. Vessels that could not support fittings were trimmed and left to be sealed by the potting material. Finally, the internal volume of the neck collar was filled with a fast-acting 2-part urethane resin (Smooth-Cast ONYX FAST, SmoothOn, Inc.), covering the remaining cervical spine and spinal cord, sealing the dural sheath (Figure 33). The specimen-collar complex was left for 15 minutes to cure. 


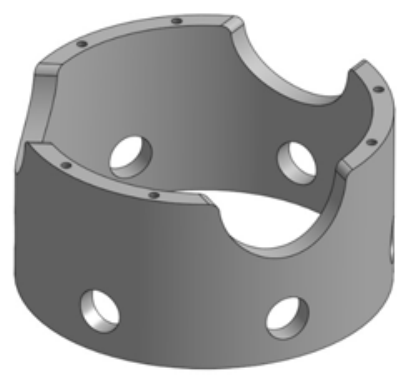

a

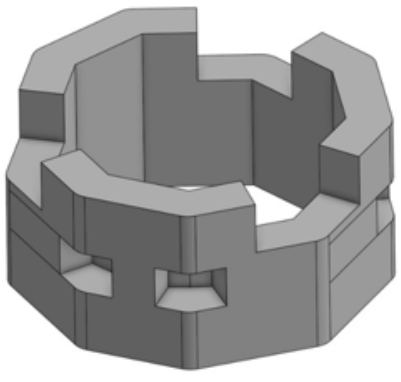

b

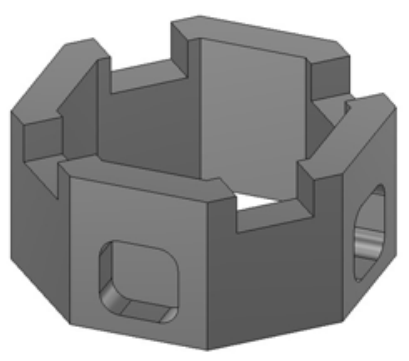

C

Figure 31: Neck collar designs. (a) First generation, aluminum tube, not MRI compatible. (b) Second generation, urethane resin, not radially symmetric. (c) Third generation, urethane resin, radially symmetric. A set of dimensioned drawings is available in Appendix B.

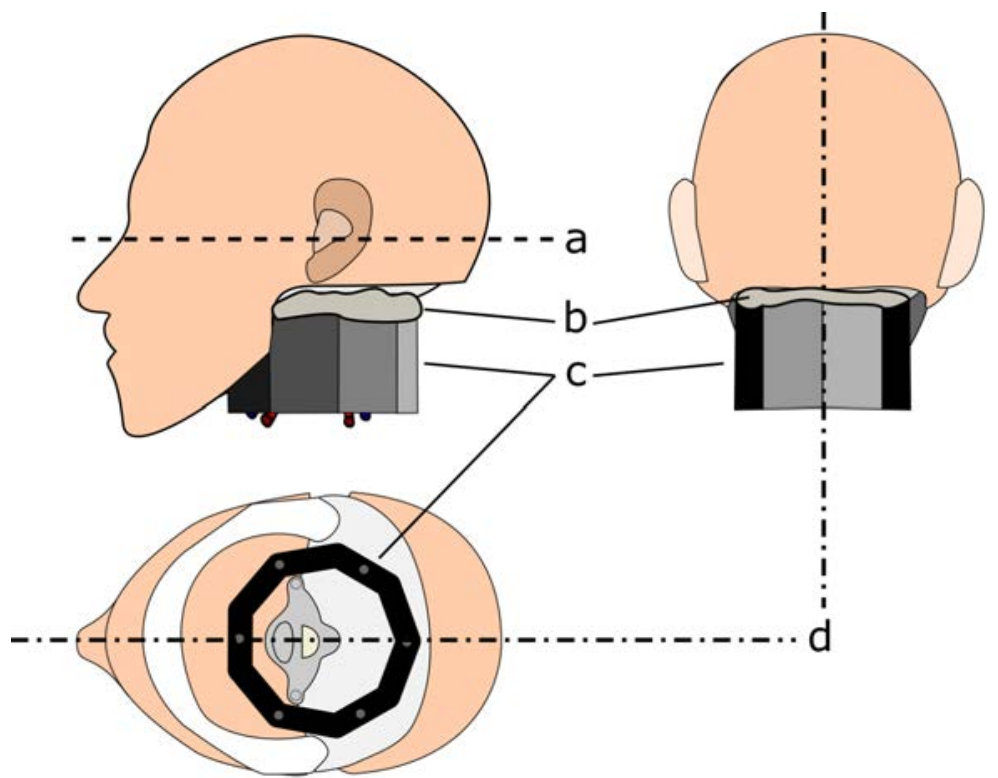

Figure 32: Alignment of neck collar on PMHS specimen. Each collar (c) was placed around the exposed vertebrae, and aligned horizontally with the Frankfort Plane (a) and the sagittal plane (d). Modelling clay (b) was used to support and seal the collar edges. 


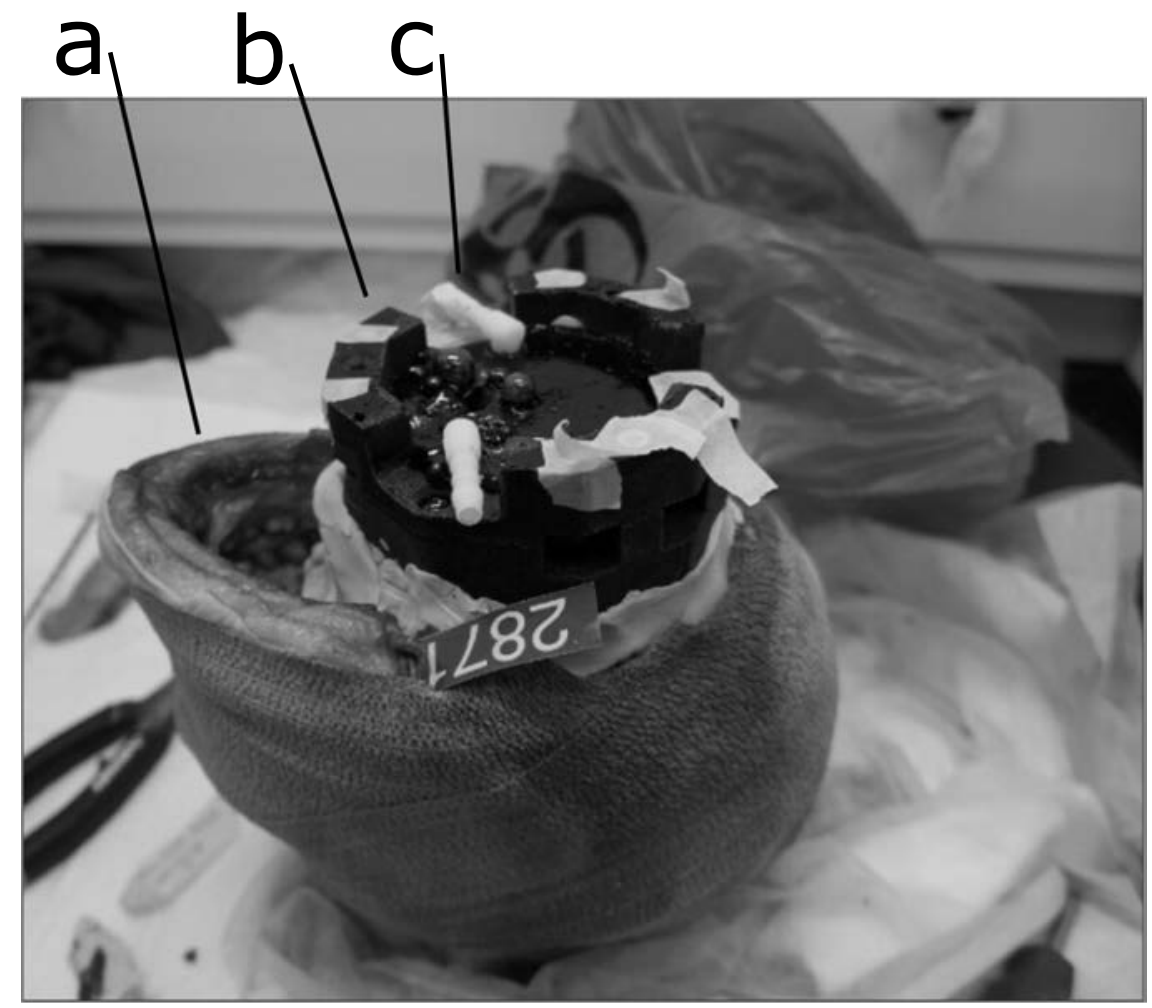

Figure 33: PMHS specimen (a) with second generation collar (b) mounted in place. Vasculature fittings (c) protrude from the collar. 


\subsubsection{Instrumentation}

With the collar secured to the specimen, instrumentation could be performed with repeatable processes using pre-configured jigs and mounting plates. The specimen was mounted upright to a two-axis positioning table, equipped with an adjustable vertical tool stand, allowing for 3-degree-of-freedom positioning of tools relative to the specimen. Once mounted, the nasal bone and occipital protuberance were used as landmarks to ensure the sagittal alignment of the specimen with the axis of the table. Coronal and axial alignment were accounted for during the collar mounting process.

Using a scalpel, a series of flaps was cut into the scalp to expose the frontal and parietal bones, as well as the coronal and sagittal sutures (Figure 34) which were used in localizing the insertion points for the pressure transducer(s), marker insertion, and perfusion fittings. Positions were cross-referenced with coordinates from tomographic images from the preliminary MRI. Each hole was drilled with a depth-limited twist-drill driven by a hand-held drill, corresponding to the required diameter. Holes were drilled normal to the surface of the skull at each point. All holes were subsequently tapped with threads to facilitate reliable mounting of fittings or plugs. Figure 35 shows the position and sizes of holes used for each purpose.

Pressure measurement was achieved through the use of one to two pressure transducers (Transducer: 105C02, $f_{R}=250 \mathrm{kHz}$, Range: $690 \mathrm{kPa}, P_{m} a x=1720 \mathrm{kPa}$, Signal conditioner: 482C05. PCB Piezotronics, Inc.) mounted at the coup, and when possible, contre-coup positions. For Specimen 2871 only a single pressure transducer was used as the second transducer had been damaged when testing 

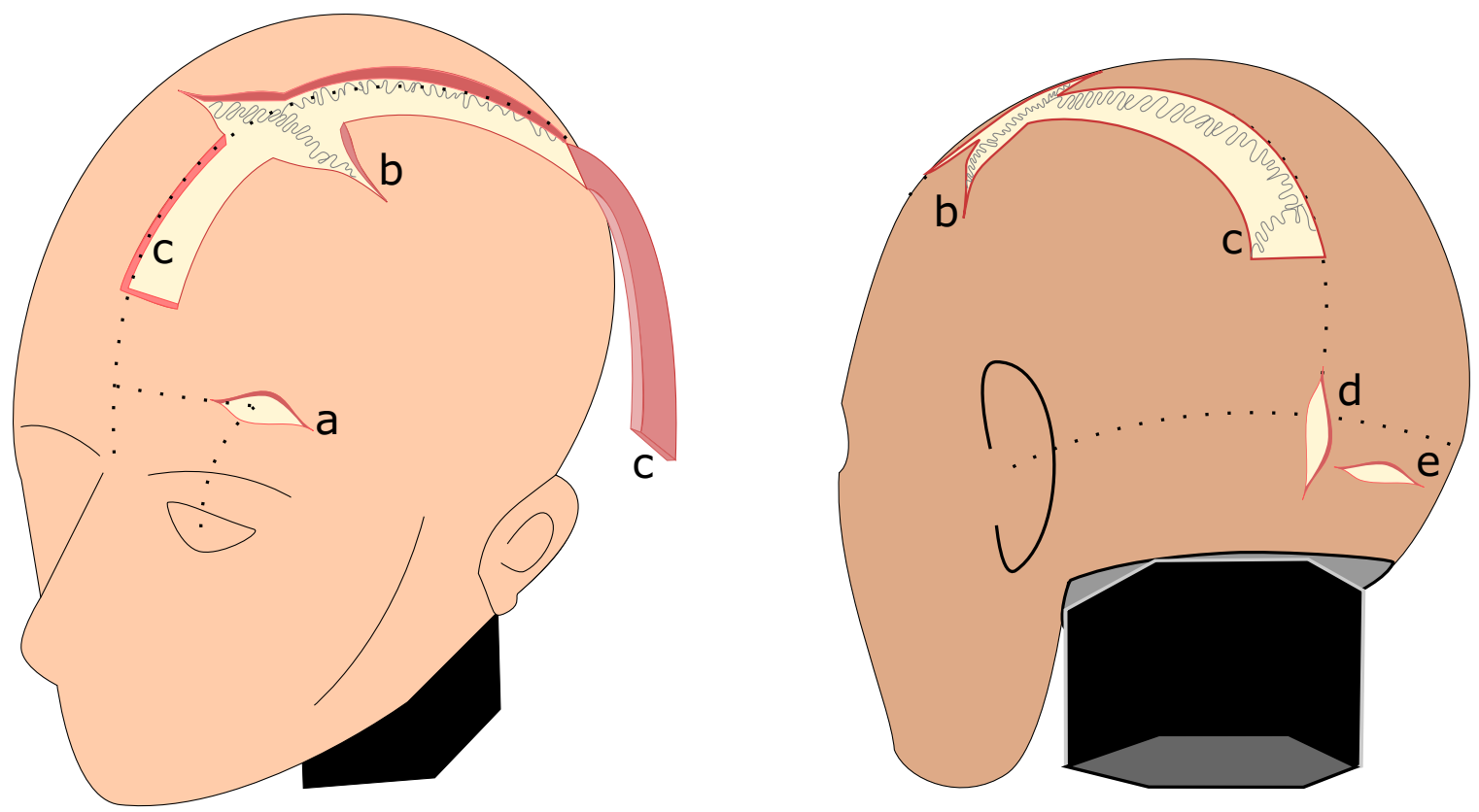

Figure 34: Series of flaps cut into scalp to access the skull bones. (a) $2 \mathrm{~cm}$ centred above left orbital. (b) $6 \mathrm{~cm}$ along coronal suture. (c) $4 \mathrm{~cm}$ above nasal bone, along sagittal plane, exposing sagittal suture up to lambdoidal suture. (d) at occipital protuberence. (e) $2 \mathrm{~cm}$ right of occipital protuberence.
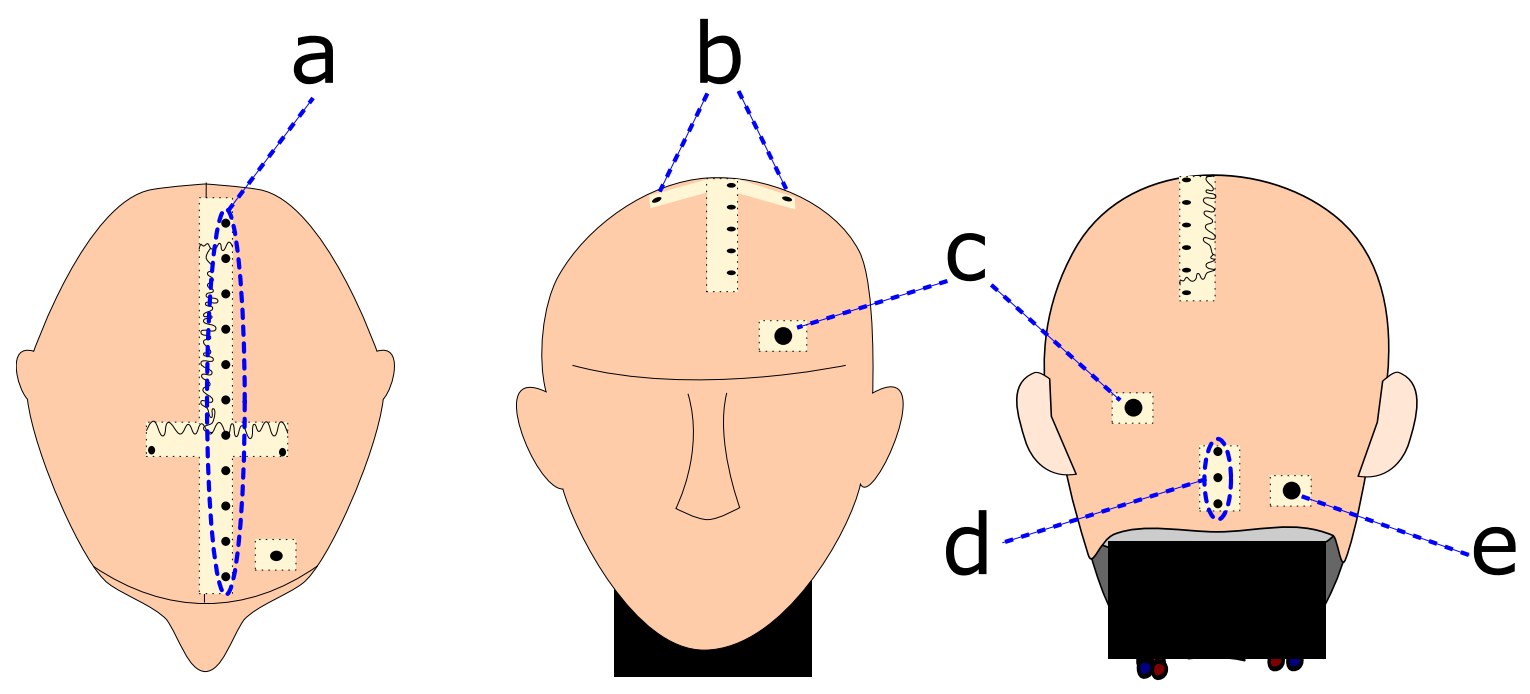

Figure 35: Position of holes drilled into skull. (a) $3 \mathrm{~mm}$ diameter marker insertion holes, $15 \mathrm{~mm}$ lateral of sagittal suture. (b) $3 \mathrm{~mm}$ holes at Kocher's point ventrical access. (c) $6.75 \mathrm{~mm}$ pressure sensor mounting holes. (d) $3 \mathrm{~mm}$ brainstem marker insertion holes, aligned with the sagittal plane. (e). $8.4 \mathrm{~mm}$ Perfusion fitting hole. 
Specimen 2842. Transducers were mounted within nylon sleeves, which were secured to the skull through tapped threads in the bone. The nylon sleeves were used to provide a layer of high-frequency vibration protection from the housing of the transducers as well as mitigate the risk of skull fracture around the large holes.

\subsubsection{Contrast Marker Insertion}

With the specimen mounted to the positioning table and its alignment checked for planarity to the table axis, drilling of the contrast marker insertion holes was performed. This step was separate from the instrumentation hole drilling process, as a depth adjustable, tool-stand mounted rotary tool (Figure 36) was used to create the access holes ( $3.0 \mathrm{~mm}$ diameter), which were drilled in the axial direction, not normal to the skull surface. The MRI-based target map was used for localization of the individual markers, relative to external anatomical features. An origin point was set at the intersection of the coronal and sagittal sutures. The primary marker plane was set a pre-determined distance from the mid-sagittal plane as to avoid placing markers too close to the falx cerebri and ventricles. This distance was informed by tomographic images. Additional target planes were established in order to place markers in the corpus callosum and brain stem. All access holes were drilled before insertion of markers began to avoid accidental alteration of the tool alignment. Although placement arrangements varied from specimen to specimen, approximately 75 markers were inserted in the regions and planes of interest, with grid-like spacing of approximately $15 \mathrm{~mm}$. This spacing was chosen as it results in a high quantity of discrete markers without limiting the integrity of the skull due to perforation. Further, this spacing is suitable for the image tracking algorithm used. At the time of this writing, only sagittal planes have been of interest and no attempt has been made to place markers in a coronal plane. The process 
for inserting markers in a coronal plane will follow that of the sagittal plane, with the specimen rotated $90^{\circ}$ on the positioning table. Upon completion of the hole drilling, marker insertion commenced. Details on the development and composition of the markers used are covered in Chapter 4 .

Similar to the rotary tool used in the drilling process, the marker insertion apparatus was mounted to the vertical tool-stand. The tool comprises a computercontrolled $200 \mathrm{~mm}$ stroke electronic linear actuator (P16-200, Actuonix Inc.), to which a custom-built cannula clamp is attached. The cannula clamp holds a guide cannula, which guides the insertion cannula. Two stylets, one pointed, and one flat-ended are also used in the process. The features of this apparatus can be seen in Figure 37

Once the tool was mounted, its alignment in with the specimen and positioning table was confirmed. The actuator was set to its maximum extension. The guide cannula, constrained in all but the vertical axis by the clamp, was inserted through the access hole in the skull into the tissue as deep as possible. To prevent coring of the tissue, the insertion cannula and pointed stylet were sleeved into the guide cannula for this step. Once the maximum depth was reached, the clamp was tightened, fixing the guide cannula to the actuator, and the insertion cannula and stylet were removed.

Using the previously generated target map, the offset distance from the position of the guide cannula at its maximum depth to the most inferior target could be calculated. The position was adjusted using the actuator control software, retracting the guide cannula the specified distance, leaving its opening at the target position. The insertion cannula was used to pick a single marker from the marker sheet (Figure 38), leaving the marker flush with the inside of the cannula. The 


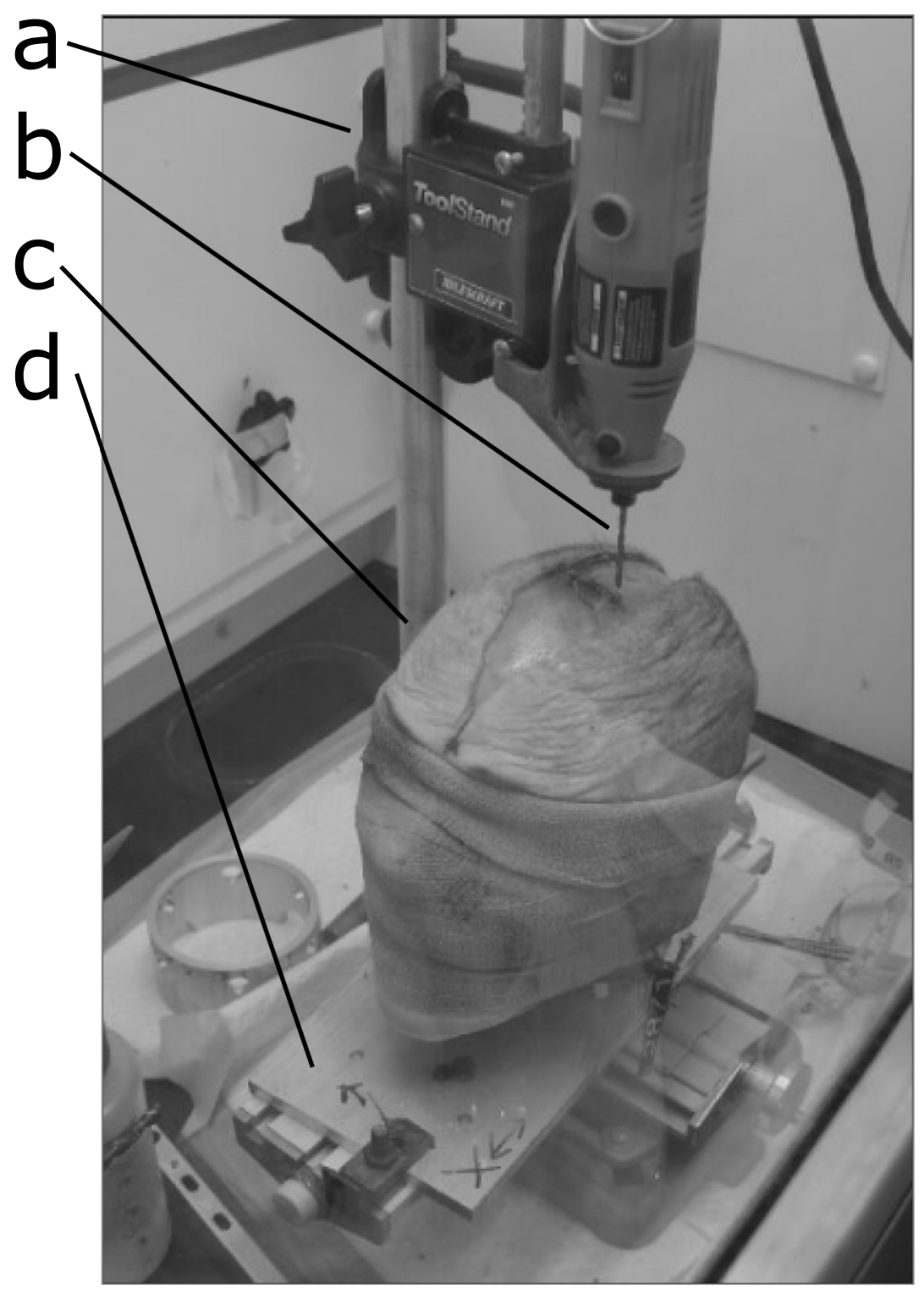

Figure 36: PMHS Specimen mounted to drilling system. (a) Height-adjustable tool stand. (b) Drill with $3 \mathrm{~mm}$ drill bit. (c). PMHS specimen, fixed to (d) X-Y positioning table. 


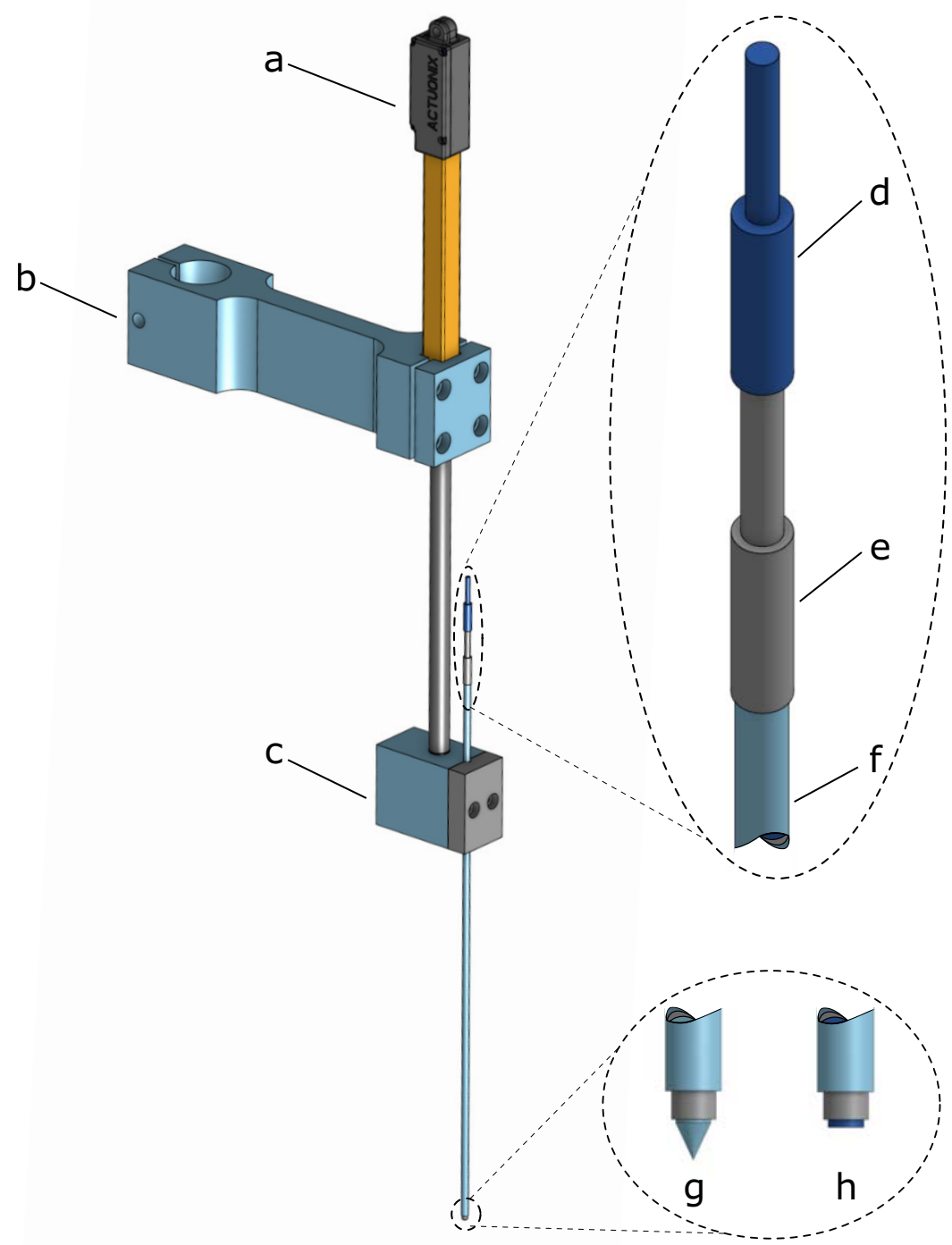

Figure 37: Marker insertion tool. (a) Electronic linear actuator. (b) Tool stand mount. (c) Cannula mount with clamp. (d) Stylet. (e) Insertion cannula. (f) Guide cannula. (g) Stylet probe tip. (h) Stylet plunger tip. A dimensioned drawing is available in Appendix B. 


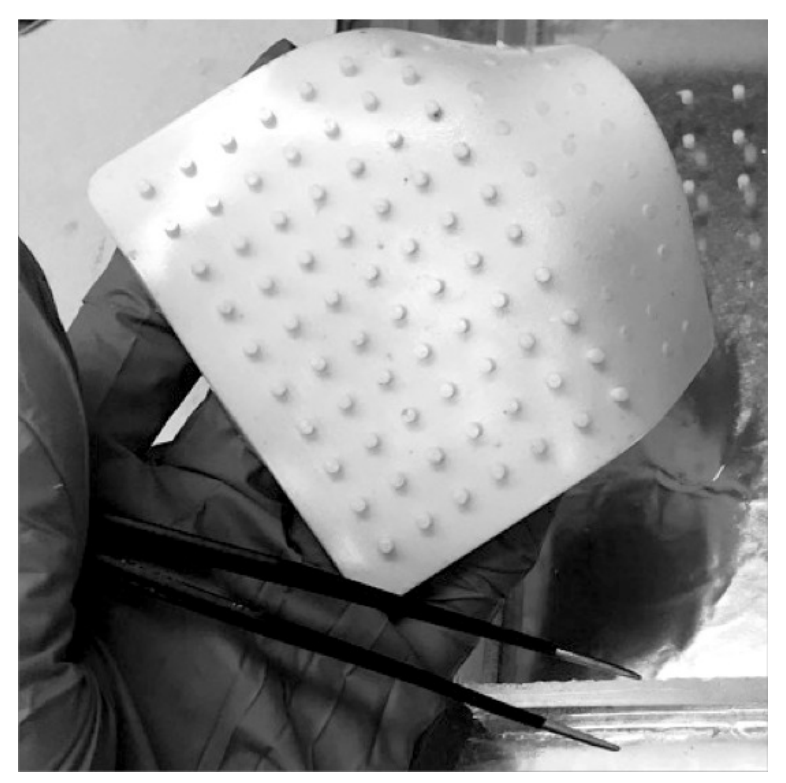

Figure 38: Sheet of contrast markers, as produced by mould. Individual markers protrude from sheet base and are removed with an insertion cannula.

insertion cannula was passed into the guide cannula, marker-end first, to its maximum depth, which was limited to ensure it would stop at the same depth as the guide. A flat-ended plunger, was inserted into the insertion cannula to poke the marker from the tip of the insertion cannula into the tissue. The plunger was also depth limited to ensure that the markers would not be pressed further into the tissue than desired.

From the new position, the offset distance for the subsequent marker was determined, and the position of the guide cannula was adjusted using the actuator. This process was repeated for each marker in the column, until the most superior marker was placed. Once completed, the clamp was loosened, guide cannula removed, and the specimen was advanced on the positioning table to the next access hole. This continued until all markers were inserted. Once all markers were inserted, holes were plugged with nylon screws. The entire process is outlined visually in Figure 39 

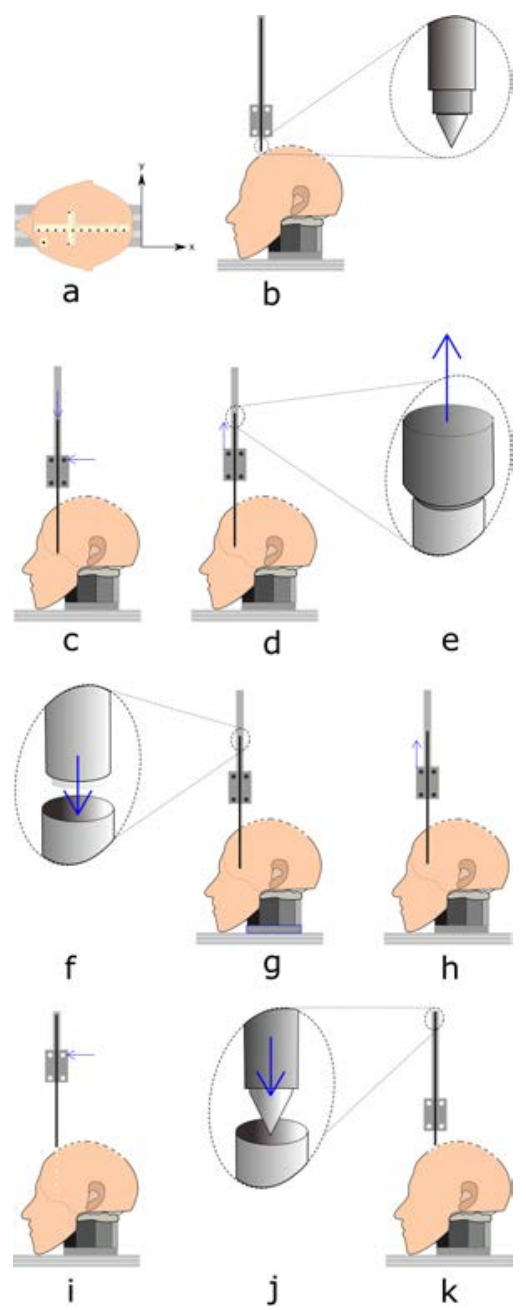

Figure 39: Marker insertion process. (a) Specimen is aligned on positioning table. (b) Probe is aligned above first hole, with pointed stylet in insertion cannula and actuator at lowest position.(c) Probe is inserted to bottom-most position and clamp tightened. (d) Actuator adjusted to first marker position.(e) Insertion cannula and stylet removed from guide cannula. (f) Insertion cannula with marker inserted into guide cannula. (g) Plunger pressed into insertion cannula to deposit marker. (h) Actuator position adjusted to next marker position. (d) through (h) repeated for all markers. (i) Clamp loosened and guide cannula removed. (j) Insertion probe placed into guide cannula. (k) Specimen advanced to next position to repeat the process. 
Approximately 80 markers were inserted in Specimen 2842, with a spacing of $\approx 1 \mathrm{~cm}$. As informed by the analysis of Specimen 2842, and in efforts to minimize experimental preparation time, markers in Specimen 2871 were placed approximately $1.5 \mathrm{~cm}$ apart, resulting in approximately 60 discrete tracking points. Markers used for displacement computation were indexed numerically in HSXR images. Figures 40 and 41 show the numbering for markers in Specimen 2842 and 2871 , respectively.

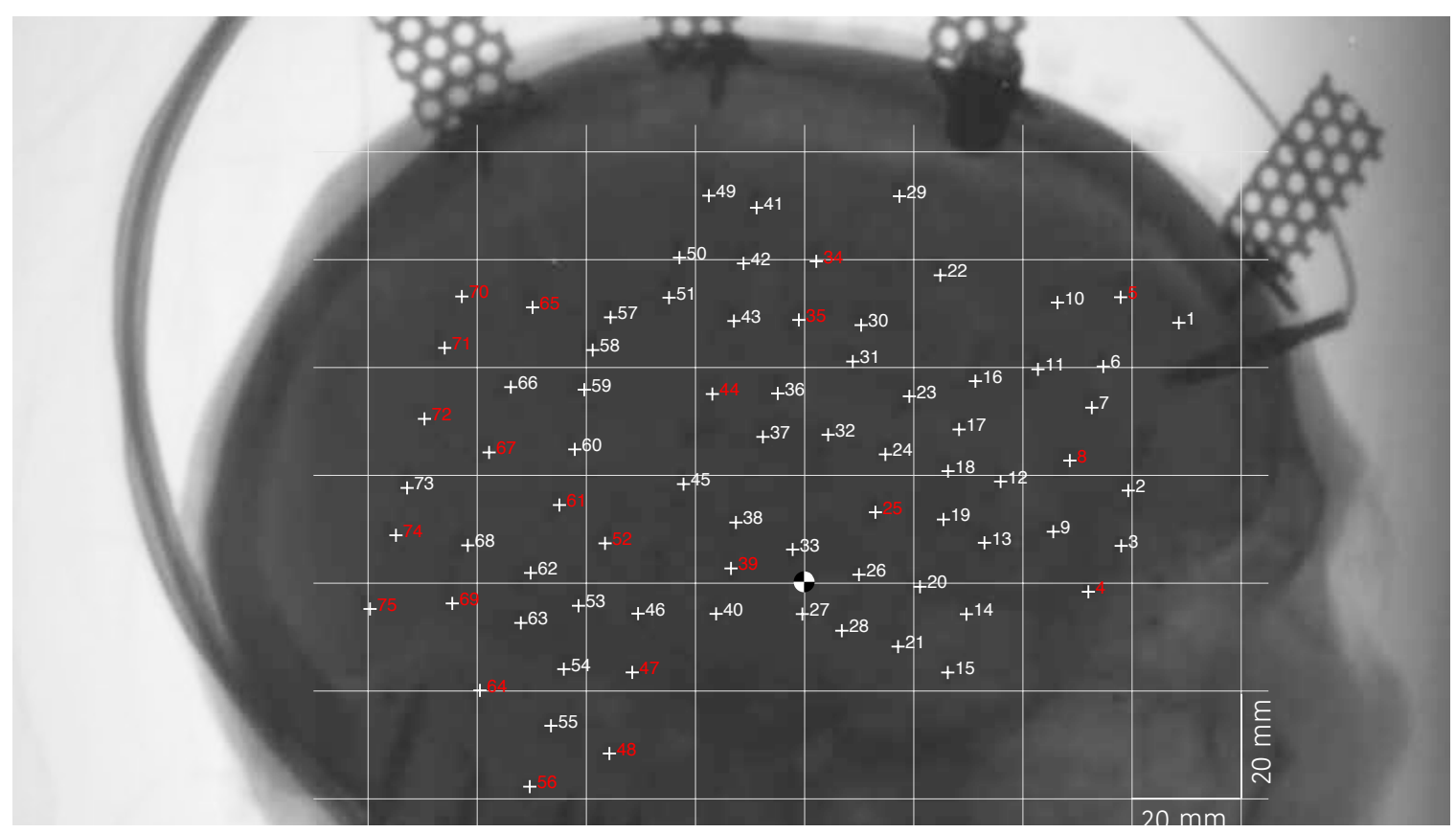

Figure 40: Marker number index for Specimen 2842. Marker placement is shown relative to the CG, which is marked for clarity at the origin of the reference grid. Markers were inserted with an approximate spacing of $1 \mathrm{~cm}$. A preexperiment MRI was used to inform the targeted locations for insertion. All markers are in a single plane. Marker numbers highlighted in red were omitted from analysis due to tracking complications or poor tissue embedment. 


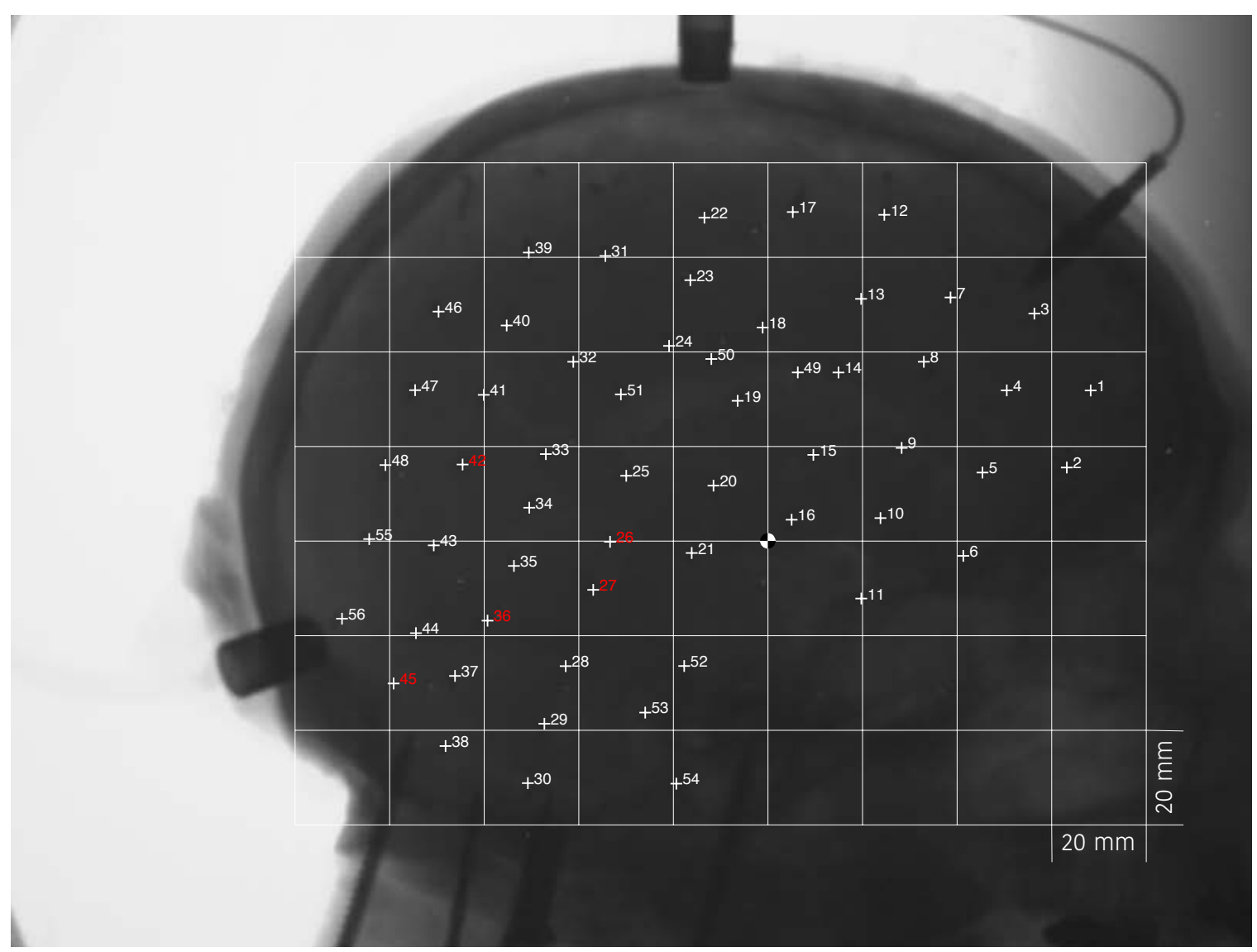

Figure 41: Marker number index for Specimen 2871. Marker placement is shown relative to the CG, which is marked for clarity at the origin of the reference grid. Markers were inserted with an approximate spacing of $1.5 \mathrm{~cm}$. A pre-experiment MRI was used to inform the targeted locations for insertion. Markers 49-51 and 52-54 are not in the primary plane, and are embedded in the corpus callosum and brain stem, respectively. Marker numbers highlighted in red were omitted from analysis due to poor tissue embedment. 


\subsubsection{Artificial Perfusion}

Artificial perfusion of the specimens was necessary to displace any entrapped air or gasses that may have been entrapped during the previous processes. Artificial cerebrospinal fluid (aCSF) was prepared in advance of receiving each specimen as per the formula found in Appendix A. The aCSF was pumped into the specimen in three stages, one each for the vasculature, ventricles, and subdural space. Once the internal spaces were filled with aCSF, a separate system maintained a constant perfusion pressure of $1000 \mathrm{mmH}_{2} \mathrm{O}$ during impact testing.

Using the fittings attached to the vasculature during the mounting process, a small hand pump was fitted to one of the carotid arteries. Fluid was pumped into the vessel until other vessels leaked fluid. As the vasculature typically contained much of its original fluid, a minimal amount was necessary to prime this system. Once fully primed, all vessels were connected together with couplers, creating a closed system.

Filling the ventricle space required accessing the lateral ventricles from the superior surface of the skull. Using a common ventriculostomy method, $2 \mathrm{~mm}$ diameter, $10 \mathrm{~mm}$ long cannulae were inserted into the left and right ventricles through access holes made at Kocher's point [63] (see Figure 35]) and temporarily fixed in place using modelling clay. One of these cannulae was used as a vent, while the other was used to fill the space with aCSF. Fluid was pumped into the space until it leaked from the vent cannula. The ventricular system is typically less than $100 \mathrm{~mL}$ total volume, however approximately $200 \mathrm{~mL}$ of fluid is needed to prime the ventricles while evacuating all gases from the space. A slight rotation and agitation of the specimen was performed to ensure that all of the gasses escaped from the space. With the ventricle space filled, these cannulae were removed and the holes plugged with nylon screws. 
Subdural spaces were filled last. Push-to-connect tube fittings $\left(1 / 4^{\prime \prime}\right.$ tube, $1 / 8-27$ NPT) were installed in the occipital and parietal bones acting as inlet and outlet fittings, respectively. These fittings were positioned such that they would be at the lowest and highest points on the specimen when it was mounted in its impact position. Fluid was added to this space after the specimen was mounted to the impact system as to reduce the risk of a leak. A $2000 \mathrm{~mL}$ reservoir of aCSF was mounted to the laboratory wall, at approximately $1.0 \mathrm{~m}$ above the center of gravity of the specimen and connected to the occipital inlet fitting via a fluid-charged tube. A vacuum line was connected to the parietal fitting. To fill the space, fluid was flowed into the occipital fitting and gases pulled from the parietal fitting. Vacuum pressure was released when the fluid was seen to rise out of the parietal fitting into the tube. Once fully charged, the specimen was left to rest at the hydrostatic fluid tank pressure of $1000 \mathrm{mmH}_{2} \mathrm{O}$. Between impacts, fluid levels were checked and replenished as needed to maintain a regulated pressure for each impact. The subdural perfusion system is shown in Figure 42 . 


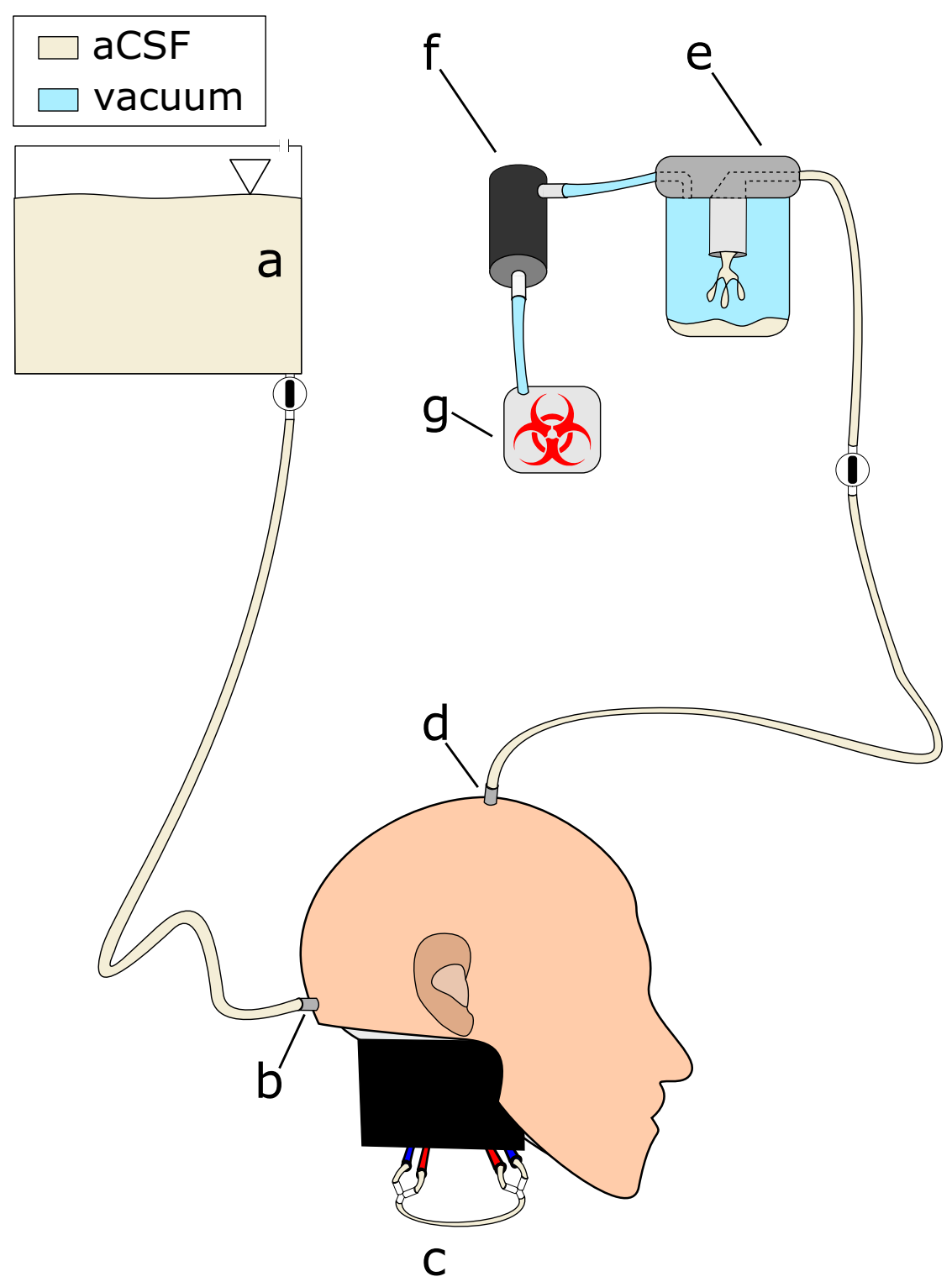

Figure 42: Artificial perfusion system used in impact setup. (a) aCSF reservoir. (b) aCSF inlet through occipital fitting (c) Vasculature bridging, primed with fluid in advance. (d) Superior vent, connected to vacuum system. (e) Vacuum reservoir. (f) Vacuum pump. (g) Gas vent through sanitizing filter. 


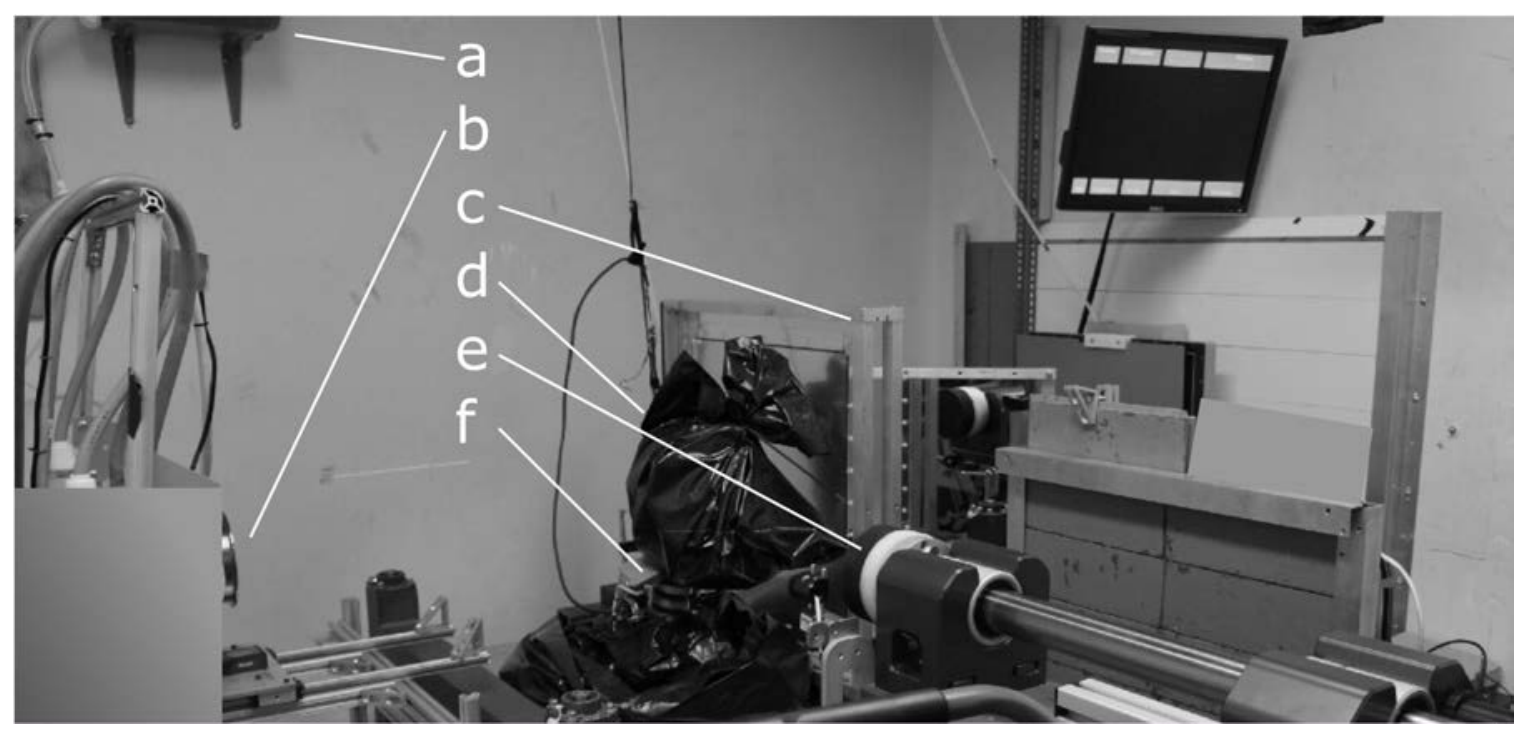

Figure 43: Impact system and experimental configuration. (a) Perfusion reservoir. (b) X-Ray source. (c) HSXR capture system. (d) PMHS specimen, bagged. (e) Linear impactor. (f) Kinematic sensor package attached to unbiased neck.

\subsection{Impact Tests}

Impact testing was performed in the HSXR Impact Laboratory following the instrumentation process. Before impacting could begin, specimens underwent preliminary imaging and mounting to the impact system, including the addition of kinematic sensing equipment. To prevent fluid leakage or aerosol generation from the impacts, heavy-duty 3-mil (0.003" thickness) poly-plastic bags were sandwiched between the specimen and any attached system, with access for component adjustment permitted only through a resealable opening. Embedded instrumentation was connected to the data acquisition system and final perfusion system connections were made. Each specimen was subject to a series of impacts based on a pre-determined protocol, which describes the stiffness of the impactor and impact speeds. The complete impact system is outlined in Figure 43 


\subsubsection{Preliminary Imaging}

Radiographic projections were used as a reference for initial marker positions, to confirm successful filling of the ventricles, and to determine the centre of gravity $(\mathrm{CG})$ of the specimen. High resolution radiographic images of the sagittal and coronal planes were captured using a clinical imaging panel (Varex Imaging, 4343DXV) at a power level of $50 \mathrm{kV}$ and $80 \mathrm{~mA}$ for $250 \mathrm{~ms}$ using the HSXR source. Sample projections can be seen in Figure 44. The CG of the specimen was computed as per the method described in Chapter 3.

In addition to the orthogonal projections mentioned above, for some specimens a complete $360^{\circ}$ set of projections was captured at $1^{\circ}$ increments using the same panel and X-ray source. Rotation of the specimen was achieved through the use of a high-precision indexed rotary table (RT-5, Newmark Systems, Inc.). By collecting this set of images, tomography could be generated using an in-house conebeam computed tomography (CBCT) algorithm. Though helpful in visualizing the marker placement, this process was time-consuming and yielded similar results to the already in-place MRI protocol, and as such was not performed for each specimen. 


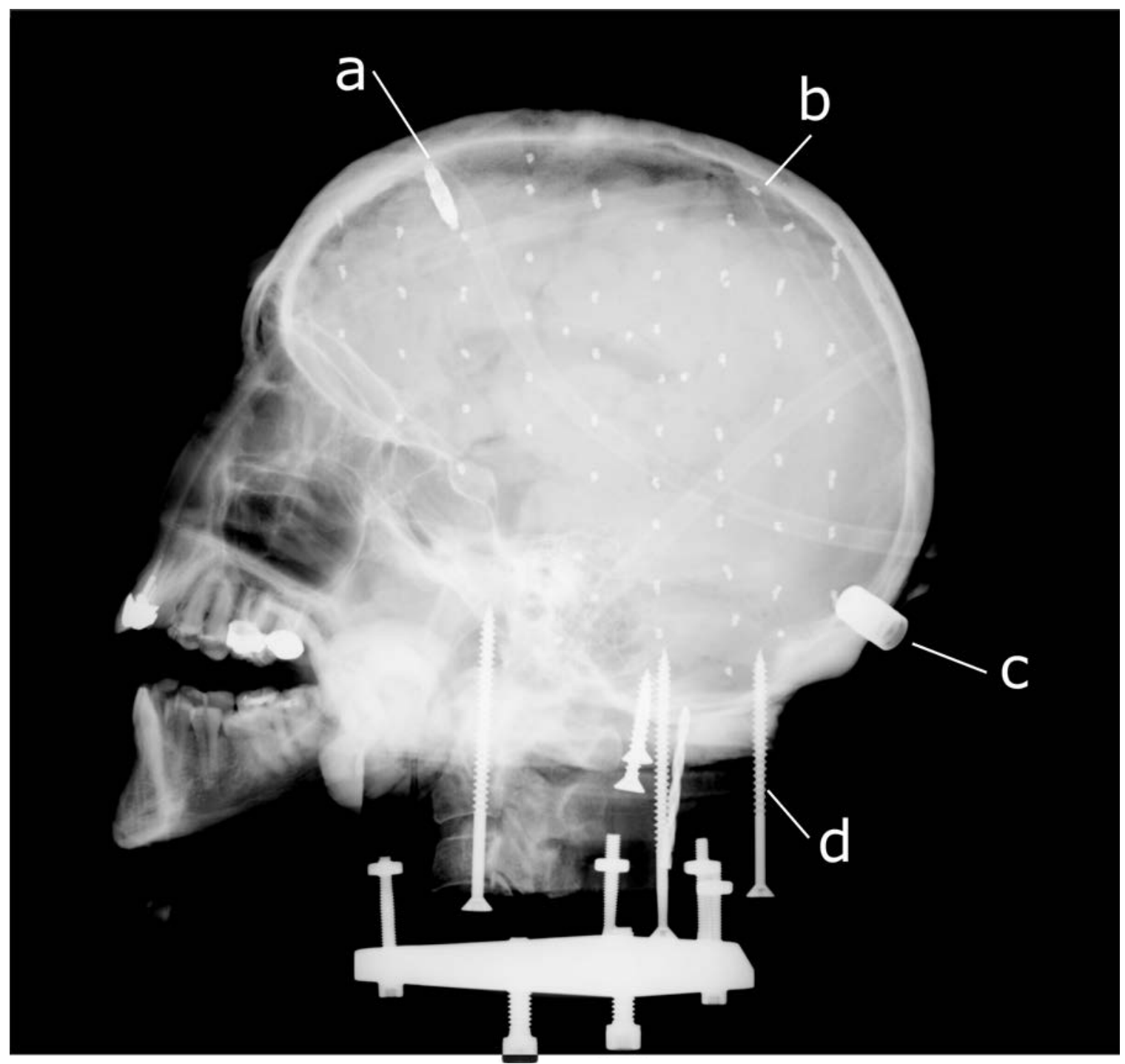

Figure 44: High resolution projection of intrumented PMHS. (a) Pressure transducer. (b) Primary marker grid. (c) Perfusion inlet. (d) Neck collar mounting screws. 


\subsubsection{Kinematics and Data Logging}

The kinematic response of the impact was captured by a 3-axis linear accelerometer, coupled with a 3-axis angular rate sensor (6DX PRO 2k-18k SLICE, Diversified Technical Systems Inc.). The sensor package was rigidly fastened to the first disk of the unbiased neck, which itself was rigidly connected to the specimen. Offset distances to the CG of the specimen were computed from the preliminary highresolution projections. Data from the accelerometer array was collected at $20 \mathrm{kHz}$ and filtered using a CFC180 filter ( $300 \mathrm{~Hz}$ low-pass) and processed using DTS Sliceware software. To compute angular acceleration from angular rate, a smoothing spline was first fitted to the acquired signal and the time derivative was taken of the smooth curve. The acceleration measurements were transformed from the point of measurement to the specimen CG as a post-processing step through the kinematic relationships shown in Equation 9

$$
\vec{a}_{A}=\vec{a}_{B}+\left(\vec{\alpha} \times \vec{r}_{A / B}\right)+\left(\vec{\omega} \times\left(\vec{\omega} \times \vec{r}_{A / B}\right)\right)
$$

where $a_{A}$ is the measured linear acceleration at the accelerometer, $\alpha$ is the derivative of the smoothed angular velocity, $\omega$, and $r_{A} / B$ is the offset distance to the specimen CG.

Signal from the pressure trandsucers was acquired by a USB Oscilliscope (PicoScope 4824, Pico Technology Ltd.) connected to a laboratory computer. In addition, two timing signals were acquired by this system. All signals were recorded at $40 \mathrm{MHz}$ and subsequently re-sampled at $20 \mathrm{kHz}$. The first of these signals came from the timing gate on the linear impactor and the second from an activity sensor attached to the HSXR system. These signals allowed the results from all different sources to be aligned temporally during the data analysis phase. 


\subsubsection{Impacts}

A pre-determined protocol for impacts was followed for each specimen. The complete set of planned impacts for the entire study can be found in Appendix C Impacts were carried out by a pneumatically driven linear impactor (Cadex Inc.), equipped with an impact ram weighing $13 \mathrm{~kg}$. Impactor speed was controlled electronically through regulation of the air pressure used to charge the system and validated with an optoelectronic timing gate. Triggering the impactor generated the control signal for the entire data acquisition system, including the HSXR imaging setup, which was calibrated to capture the entire impact event. Impactor stiffness was varied by changing the striking surface material, or impactor cap. Three different circular impactor caps with a diameter of $13 \mathrm{~cm}$ were created for low, medium, and high stiffness. Impactor cap properties are listed in Table 7 The response time is indicative of the stiffness of the cap, with lower stiffness resulting in a longer duration of contact.

Table 7: Impactor cap stiffness

\begin{tabular}{c||ccc} 
Level & Material & Thickness $(\mathrm{mm})$ & Response Time $(\mathrm{ms})$ \\
\hline Low & VN600 & 76.2 & 25 \\
Medium & VN600 & 38.1 & 15 \\
High & Neoprene & 50.8 & 5
\end{tabular}

Specimens were mounted upright to the adjustable impact table. Position adjustments were made to achieve the desired striking point of the ram as per the protocol. Specimens were positioned such that the ram would strike between $0 \mathrm{~mm}$ and $5 \mathrm{~mm}$ beyond the timing gate to minimize velocity loss due to system friction. High-contrast fiducial markers were attached to the skull along the planes containing the embedded contrast markers, making use of the existing nylons screws, and 
the angular offset of specimens was adjusted to ensure these planes were aligned with the HSXR imaging plane. Once aligned, the specimen position was secured by tightening all adjustments clamps, and subdural perfusion was completed. Signal wiring and perfusion tubing was routed out of the containment bag opening and secured to an overhead gantry. Once set, all data acquisition systems were activated and the first impact was triggered. Between impacts the system was re-set and all components were checked for alignment, damage, and leakage. Impactor velocity was increased incrementally as per the protocol. HSXR images were captured at 7,500 fps, with a pre-roll of $20 \mathrm{~ms}$ and a total recording time of $500 \mathrm{~ms}$. Complete HSXR details are seen in Chapter 3.

\subsection{Post-Experiment Procedures}

Following the execution of the impact protocol, each specimen was disconnected from the impactor, perfusion, and data acquisition systems and returned to the Biomaterials Laboratory. All externally mounted instrumentation, fiducial markers, and sensors were removed, along with the urethane neck collar and all fastening hardware. Specimens were then chilled at $4^{\circ} \mathrm{C}$ until they were transported for post-experiment MRI acquisition.

The post-experiment MRI protocol followed that of the pre-experiment protocol. Images were typically collected within 12 hours of the completion of the impacts to minimize tissue degradation and marker migration. Once imaging was completed, specimens were returned to the Anatomy Laboratory at University of Ottawa for embalming and later dissection by the neurosurgery team. Embalming was performed by staff of the University of Ottawa to minimize further tissue degradation. Tissue damage and embedded contrast marker positions were confirmed during the dissection by the neurosurgeons based on their findings and 


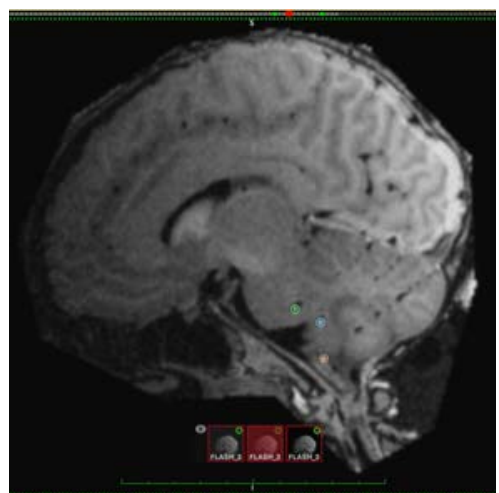

a

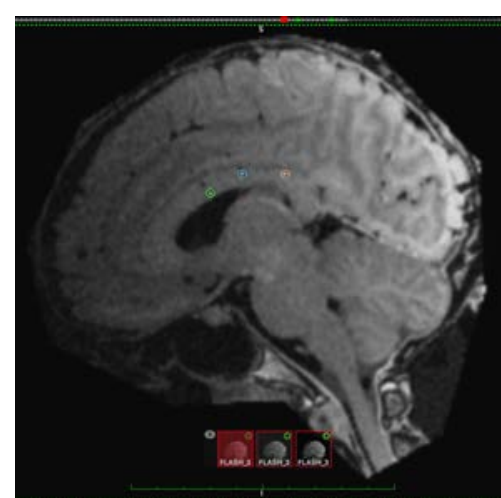

b

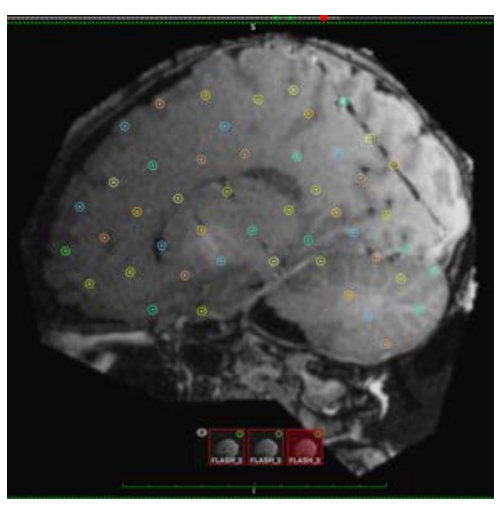

C

Figure 45: Magnetic Resonance Image showing three target marker slices after impact experimentation. (a) Brain stem markers. (b) Corpus callosum markers. (c) Primary parasagittal grid.

comparison with the post-experiment tomographic images. A sample of postexperiment images is seen in Figure 45, highlighting the position of embedded target markers. Larger images for each specimen appear in Appendix F 


\section{Chapter 6}

\section{PMHS Results and Discussion}

To add and improve upon the data available for model validation, a comprehensive experimental approach was taken to collect data during impacts performed on PMHS heads. This revised approach considers the marker design study covered in Chapter 4 , and follows the methods described in Chapter 5 , making use of the advanced HSXR system at Carleton University. The data collected from these impacts was analysed to evaluate and validate this collection protocol, ensuring that further experimentation will produce the high fidelity data needed to validate advanced models of the head and brain.

\subsection{PMHS Impact Results}

Two PMHS head specimens, numbered 2842 and 2871 respectively, were prepared and subjected to seven (7) linear impacts each in order to obtain quantitative data on the response of the head and brain. Anthropometric data for each specimen, as well as kinematic, pressure, and displacement data not discussed in this section, can be found in Appendix $\mathrm{D}$. Both specimens were impacted by the same $13 \mathrm{~kg}$ impactor ram, however Specimen 2842 was struck with a high compliance impactor cap, and 2871 was struck with a low compliance impactor cap to simulate different 
impact characteristics. Impactor cap materials, thickness, and associated impact durations are available in Chapter 5. All impacts were aligned approximately $2 \mathrm{~cm}$ above the CG of the head and struck on the anterior surface. Each specimen was pinned at the neck, limiting translation in the direction of impact, however neck flexion provided rotational freedom. Impacts for each specimen were planned per the impact protocol in Appendix C. and the final impact speeds for both specimens can be found in Table 8

Table 8: Summary of impacts for PMHS specimens

\begin{tabular}{c|cc} 
& \multicolumn{2}{|c}{ Speed (m/s) } \\
Impact & PMHS 2842 & PMHS 2871 \\
\hline 1 & 0.8 & 1.0 \\
2 & 1.5 & 2.1 \\
3 & 1.9 & 3.0 \\
4 & 3.1 & 3.4 \\
5 & 4.5 & 1.2 \\
6 & 5.1 & 3.8 \\
7 & 1.6 & 3.7
\end{tabular}

\subsubsection{Kinematic Response}

The kinematic trace for each impact was collected at $20 \mathrm{kHz}$ and low-pass softwarefiltered at $300 \mathrm{~Hz}$ (CFC180). The accelerometer package was rigidly attached to a mount, which was offset from the CG of each head. Offset distances were used to compute the resultant linear and angular velocity at the CG and are listed in Appendix D. Angular velocity was collected instead of angular acceleration. As such, a smoothing spline was fit to the data, and its derivative taken in order to compute angular acceleration. Peak resultant linear and angular accelerations for all 
impacts performed with each specimen are shown in Table 9 . For each of the specimens, Impact 6 was the highest energy, yielding the greatest kinematic response. The kinematic traces for these two impacts are shown in Figure 46. A series of X-ray video frames is also provided in Figure 47 showing the moment of impact, peak acceleration, and later return to the impact position.

Table 9: Peak resultant linear and angular acceleration

\begin{tabular}{c|cc|cc} 
& \multicolumn{2}{|c}{ PMHS 2842} & \multicolumn{2}{c}{ PMHS 2871 } \\
Impact & Linear (g) & Angular $\left(\mathrm{rad} / \mathrm{s}^{2}\right)$ & Linear (g) & Angular $\left(\mathrm{rad} / \mathrm{s}^{2}\right)$ \\
\hline 1 & 6.0 & 280 & 9.7 & 431 \\
2 & 16.3 & 613 & 48.6 & 2530 \\
3 & 19.2 & 663 & 62.2 & 3754 \\
4 & 36.2 & 1347 & 82.1 & 5031 \\
5 & 34.9 & 1629 & 22.8 & 1193 \\
6 & 54.0 & 2149 & 92.0 & 5532 \\
7 & 10.3 & 528 & 87.3 & 5284
\end{tabular}




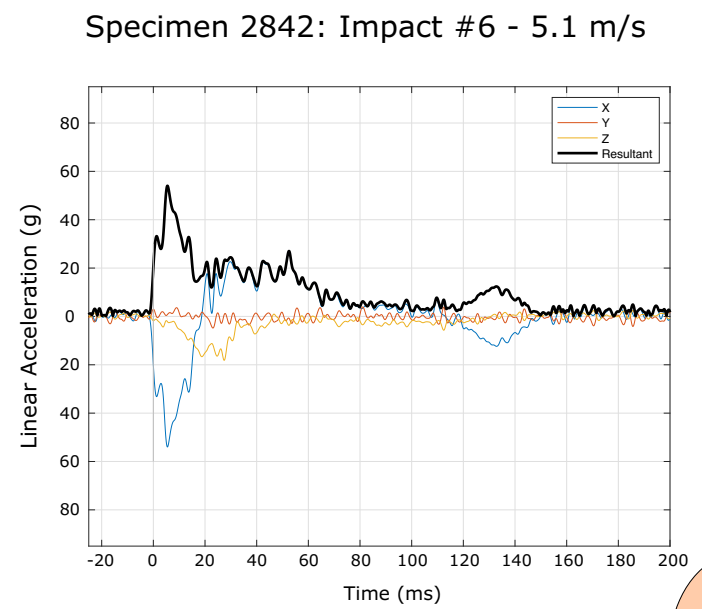

Specimen 2871: Impact \#6 - $3.8 \mathrm{~m} / \mathrm{s}$
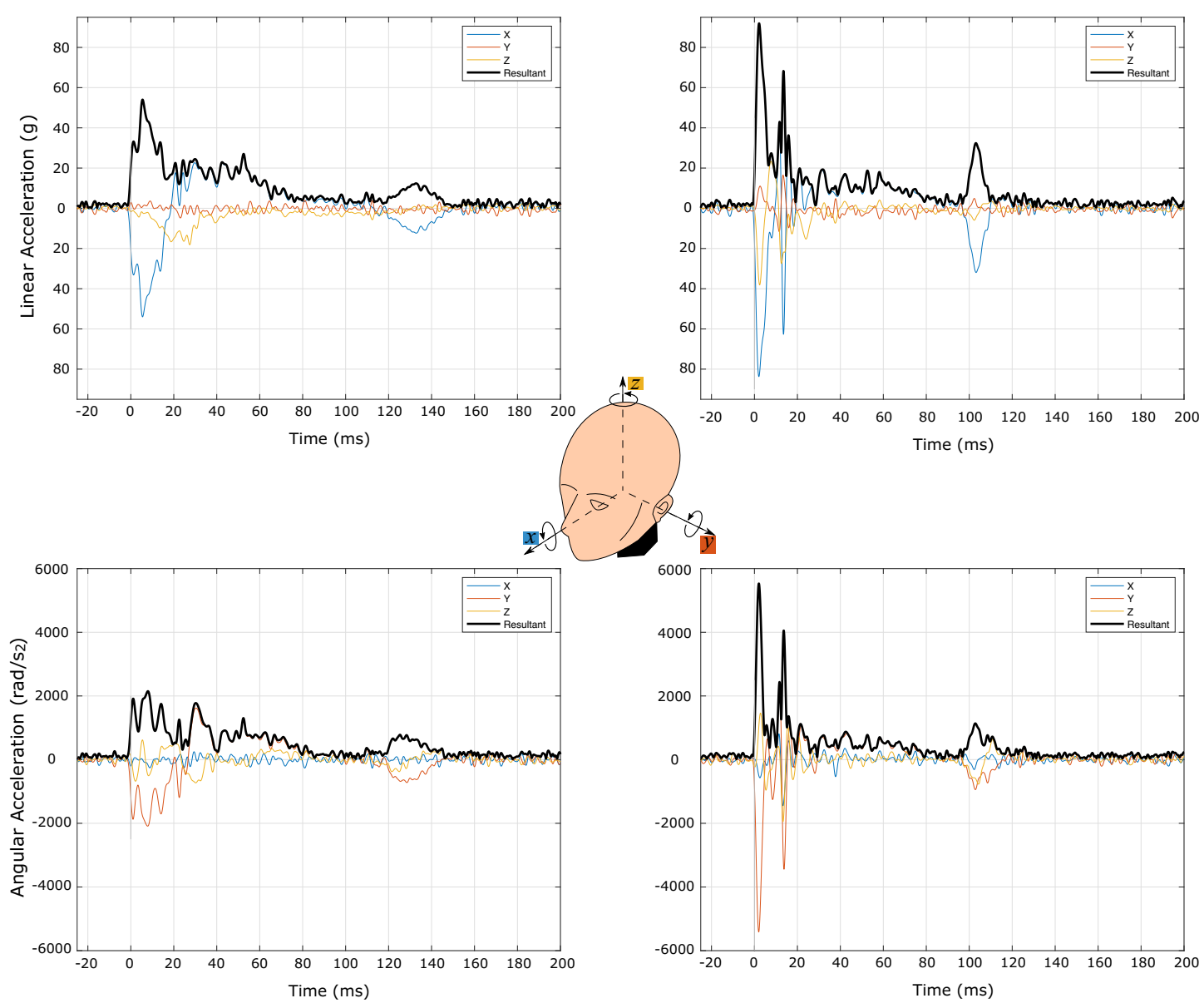

Figure 46: Linear and angular acceleration for Specimens 2842, and 2871, with impact speeds of $5.1 \mathrm{~m} / \mathrm{s}$ and $3.8 \mathrm{~m} / \mathrm{s}$ respectively. Principle directions are shown relative to the PMHS head (centre), and resultant accelerations are plotted in black. 2842 was struck with a high compliance impactor cap, reducing the intensity of the loading, but extending the duration, whereas 2871 was struck with a low compliance cap. The lower intensity signal after $100 \mathrm{~ms}$ is representative of the head returning to the impactor due to the engagement of the neck. 

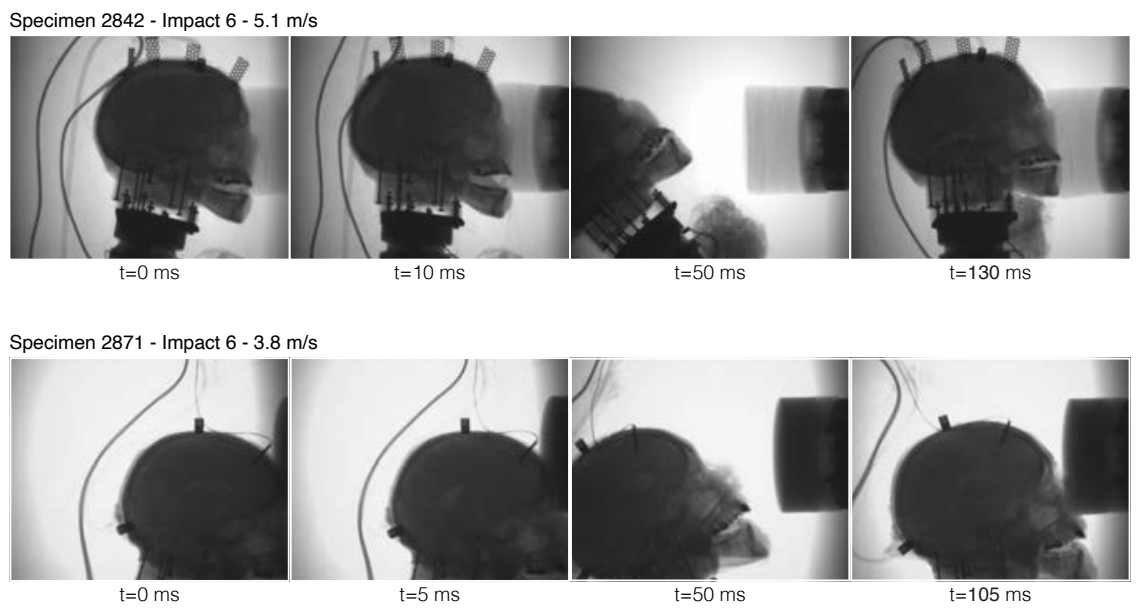

Figure 47: X-Ray images for each specimen corresponding to kinematic points of interest, including moments of impact, peak acceleration, and return to impactor. 


\subsubsection{Pressure Response}

Pressure at the impact site (coup pressure) could only be collected for Specimen 2871 due to equipment availability. Pressure was sampled at $40 \mathrm{MHz}$ and left unfiltered to ensure the capture of peaks and to avoid time shifting. An offset is present before impact as well as drifting baseline, occurring in time with the X-ray discharge. Signals were re-sampled at $40 \mathrm{kHz}$ and a second order exponential fit was applied to the drift, allowing an offset to be applied. The uncompensated and compensated signals for Specimen 2871, Impact 6 (2871-6), can be seen in Figure 69 The maximum and minimum coup pressures due to impact are shown in Table 10 with their respective time of occurrence. These values were calculated using the offset from the shifted baseline.

Table 10: Adjusted peak coup pressure - Specimen 2871

\begin{tabular}{c|cc|cc} 
Impact & Max. Pressure (kPa) & Time $(\mathrm{ms})$ & Min. Pressure $(\mathrm{kPa})$ & Time $(\mathrm{ms})$ \\
\hline 1 & 2.0 & 7.69 & -1.2 & 14.70 \\
2 & 25.7 & 4.44 & -6.7 & 10.26 \\
3 & 60.8 & 3.48 & -12.2 & 9.66 \\
4 & 79.3 & 3.83 & -7.3 & 7.99 \\
5 & 12.8 & 4.90 & -2.4 & 12.26 \\
6 & 98.4 & 3.75 & -11.7 & 10.02 \\
7 & 81.6 & 4.03 & -12.8 & 10.55
\end{tabular}




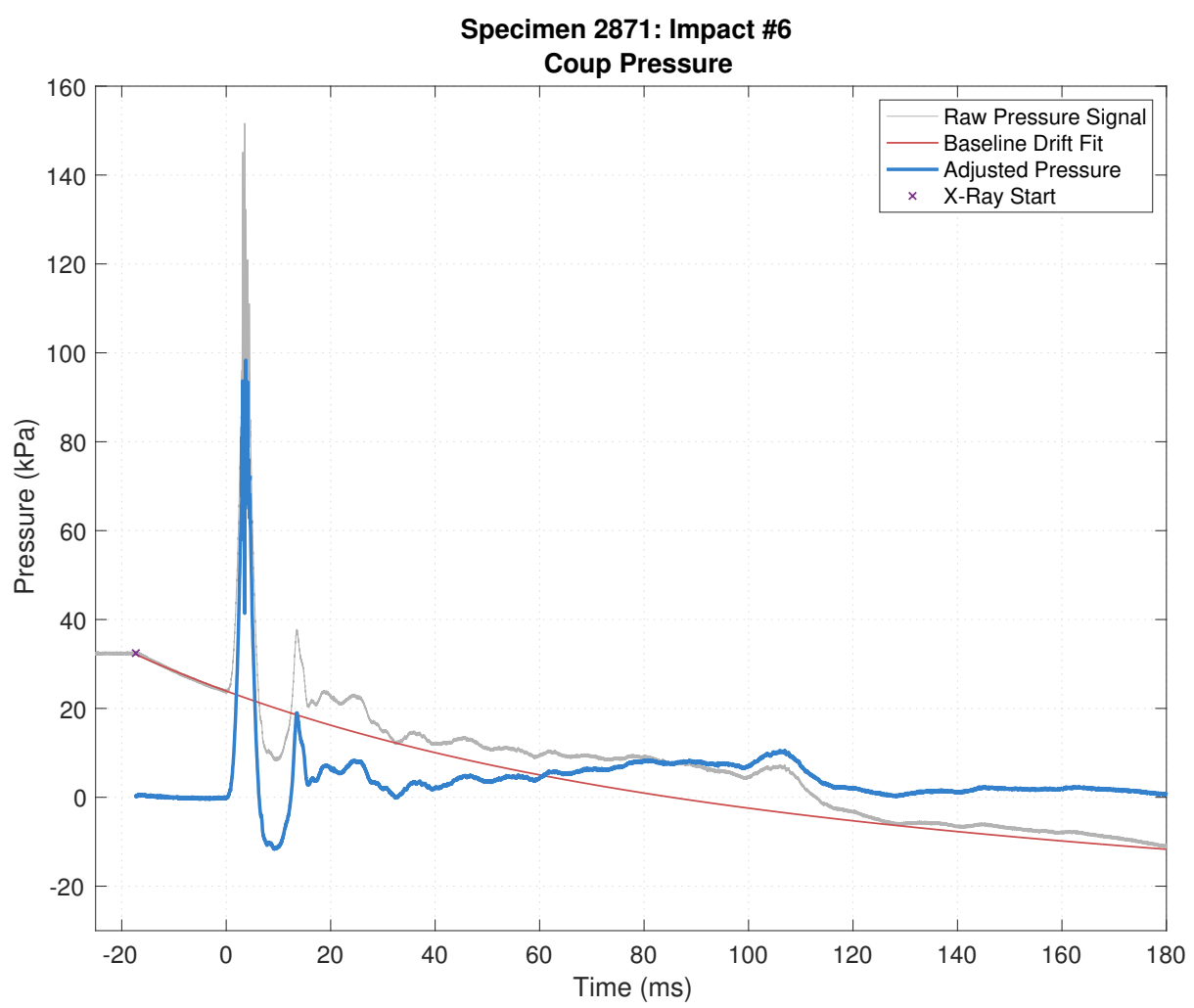

Figure 48: Coup pressure signal for Specimen 2871, Impact 6, $3.8 \mathrm{~m} / \mathrm{s}$ and associated compensated values. Pressure rises rapidly following the moment of impact to $98.4 \mathrm{kPa}$. The compensated value has been shifted from the offset at time $\mathrm{t}=0 \mathrm{~ms}$. 


\subsubsection{Brain Tissue Displacement}

High-speed X-ray videos were captured for 13 of the 14 PMHS head impacts. During the first impact on Specimen 2842, the X-ray system encountered a fault, resulting in a loss of video collection. Tracking of embedded contrast markers was performed on the resultant video files. Marker displacement, with respect to the skull, was determined for 11 of the 13 recorded impacts. Tracking could not be completed for $2842-5$ and $2842-6$ as a comparable duration was not visible in the videos. As such, they have been omitted from this analysis. These videos have been retained as their analysis may be possible with a future tracking algorithm.

The MRI images for Specimen 2871 were reviewed by neurosurgeons to confirm the location of markers within the parenchyma and identify markers that were not sufficiently embedded. Poorly embedded markers in Specimen 2842 have not yet been confirmed, as the MRI review is pending. Markers that exhibited nonconforming or migratory behaviour in Specimen 2842 have been excluded as it is likely they are not well embedded. While the tracking algorithm was able to track most markers, some remained problematic, as they could not be identified in each frame due to interference from other image features. Due to these exclusions, Specimen 2842 had 53 viable markers and Specimen 2871 had 50 viable markers. An overview of the excluded markers and reason for exclusion is available in Appendix E

Peak displacement along with the associated post-impact time, marker, and region of the brain, are available in Table 11. Marker tracking was performed in units of pixels and then converted to millimetres using distinct scaling factors for each impact (Appendix D). Considering the marker centre scatter of $0.7 \pm 0.1 \mathrm{px}$ found previously, this translates to 0.19 to $0.27 \mathrm{~mm}$ precision on marker positions, 
depending on the scaling factor used. For all traces, motion was observed to follow looping patterns as reported in past studies [25], with more pronounced displacement near the brain-skull boundary, as shown in Figure 49 . Of the impacts for which data is available, 8 are of particular interest for discussion due to commonalities between their impact characteristics. The displacement traces for these impacts are shown and discussed below (Figures 51 to 58). The remaining three displacement traces may be used for future validation work. As MRI results were available for Specimen 2871, its traces have been separated into discrete regions; Specimen 2842 traces are shown in a single region.

Table 11: Peak marker displacement

\begin{tabular}{cc|ccc|c} 
PMHS & Impact & Displacement $(\mathrm{mm})$ & Time $(\mathrm{ms})$ & Marker & Region \\
\hline 2842 & 2 & 10.2 & 104.4 & 29 & Frontal \\
& 3 & 3.1 & 99.6 & 73 & Occipital \\
& 4 & 5.5 & 84.0 & 41 & Parietal \\
& 7 & 10.9 & 124.3 & 29 & Parietal \\
\hline 2871 & 1 & 5.3 & 12.8 & 12 & Frontal \\
& 2 & 8.0 & 8.0 & 39 & Parietal \\
& 3 & 9.6 & 18.8 & 22 & Frontal \\
& 4 & 13.4 & 18.9 & 17 & Frontal \\
& 5 & 4.9 & 63.2 & 44 & Occipital \\
& 6 & 10.4 & 18.8 & 17 & Frontal \\
& 7 & 11.1 & 57.9 & 12 & Frontal
\end{tabular}




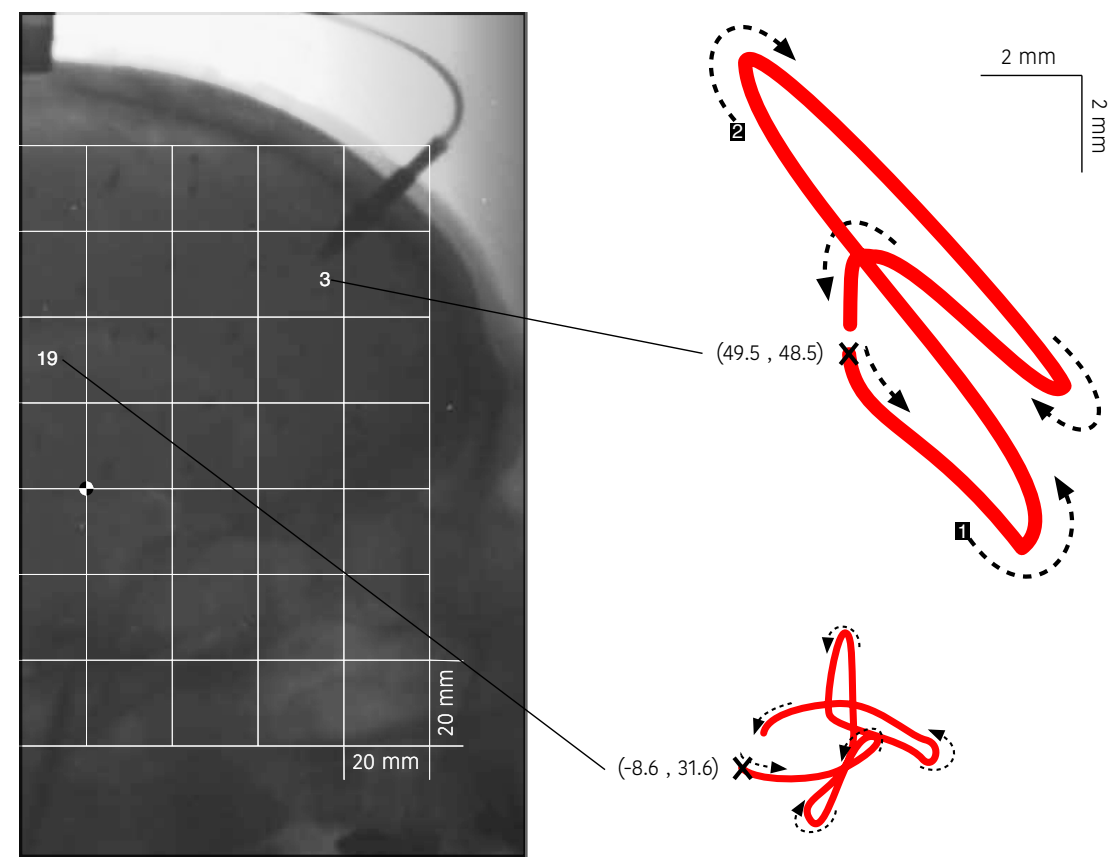

Figure 49: Smoothed motion paths of highlighted Markers, 3 and 19, in 2871-3. Dashed arrows show phases of motion (1,2 highlighted). Marker 3, further from the centre of rotation, exhibits a greater magnitude of motion. Marker 19 , while still exhibiting the characteristic looping pattern, does so at a reduced magnitude, as it is closer to the centre of rotation. These specific markers were chosen for ease of illustration and are representative of a broad trend. 


\subsection{Discussion of PMHS Data}

The discussion of collected PMHS data is broken into two parts; validation, and displacement. Due to the inherent variability in human cadaver experimentation, previous studies have reported a broad range of results. While direct comparison of results between different PMHS impact studies is challenging, validation of the present study can be discussed in terms of kinematics, pressure, and wholebrain motion trends. The primary aim of this study is to develop a comprehensive testing protocol capable of observing regional displacements within the brain. Discussion and comparison of not only the brain as a whole, but of individual regions, and sub-regions, highlights the value of this protocol.

\subsubsection{Study Limitations}

Care was taken to ensure identical preparation of each specimen, though there were minor deviations due to anthropometric differences and apparatus variations between collections. Adjustments to neck mounting, pressurization, transducer placement, and marker placement were all necessary to accommodate these differences.

Due to its smaller size, Specimen 2842 was challenging to mount to the uniform neck collar while maintaining space for perfusion fittings. Contrary to the protocol, vascular pressurization could only be performed through one side of carotid artery, while all other vascular openings were sealed with the neck potting material. Artificial cerebrospinal fluid was flowed into the system before potting to evacuate any accumulated gases; the neck potting procedure was then performed. Due to space constraints, the location of the pressure transducer used for coup pressure fell within the impact region, resulting in its destruction during the impact protocol, while the contre-coup location was not measured due to limited 
space. The methodology protocol was more closely adhered to for Specimen 2871 as the specimen was larger and its vasculature was readily separable. Due to the earlier loss of a pressure transducer (experiment with 2842), only coup pressure could be recorded.

A baseline drift is present in all collected pressure signals. The drift starts from the stable sensor offset voltage of approximately $0.201 \mathrm{~V}$ (equivalent to an offset pressure of $\approx 32 \mathrm{kPa}$ ). Impacts 1 through 3 exhibit a rising drift and 4 through 7 have a falling drift. The drift begins at the moment the X-ray trigger signal is received, and before the impact occurs. The cause of the drift is likely related to the high-energy discharge associated with the X-ray source. The piezoelectric sensor in the specimen is sensitive to voltage differentials. Post-processing revealed an approximately exponential fit to the drifting signal, implying an inductive phenomenon. Further investigation into the drift will be performed, as electronic isolation, or post-process signal correction may be suitable solutions. For the present study, values were compensated by fitting a second-order exponential curve to the drifting baseline. Resulting pressure measurements were consistent with expectations.

The displacements collected in this study are two-dimensional, as the Carleton HSXR system captures only a single projected plane. Out-of-plane motion would skew displacements at the image boundaries, however it is assumed to be negligible, as impacts were aligned with the midline of the head, the material bound on all sides, and images were collected perpendicular to the impact plane. Due to the nature of radiographic projections, significant out-of-plane motion would be visible as magnification distortion, which was not observed. Further, minimal out-of-plane accelerations were observed, suggesting mostly in-plane motion of the head itself. Future studies using high-speed bi-plane X-ray imaging will help to eliminate this uncertainty. 


\subsubsection{Validation of Data}

The kinematic responses collected for each impact are within the range of those reported in previous works. A similar study by Hardy et al., with impact speeds from 3.0 to $3.8 \mathrm{~m} / \mathrm{s}$, captured peak linear and angular acceleration between 38 and $291 \mathrm{~g}$ and 2370 and $24206 \mathrm{rad} / \mathrm{s}^{2}$, respectively [25]. This broad range of responses is due in part to trials using helmeted and unhelmeted specimens, a rigid impactor plate, and varied impact positions. In the present study, the greatest resultant linear and angular accelerations were seen in 2842-6 and 2871-6, corresponding to the maximum impact velocity for each specimen (Figure 46). Specimen 2871 was struck at $3.8 \mathrm{~m} / \mathrm{s}$, with a low-compliance impactor, resulting in resultant peak and angular accelerations of $92.0 \mathrm{~g}$ and $5532 \mathrm{rad} / \mathrm{s}^{2}$, respectively. Despite a higher impact speed of $5.1 \mathrm{~m} / \mathrm{s}$ for Specimen 2842, the high-compliance impactor resulted in reduced resultant accelerations of $54.0 \mathrm{~g}$ and $2149 \mathrm{rad} / \mathrm{s}^{2}$. The reduction of approximately $60 \%$ due to impactor compliance is coincident with the results observed by Hardy et. al, when comparing helmeted to non-helmeted impacts. By comparison, similar helmeted and unhelmeted impacts performed in the earlier study, C064-T2 and T4 [25], respectively, were performed at $3.9 \mathrm{~m} / \mathrm{s}$. These tests resulted in peak linear accelerations of $64 \mathrm{~g}$ and $122 \mathrm{~g}$. The impact speeds (Table 8) and associated peak resultant accelerations (Table 9) in the present study are consistent with the expected trends. Additional comparisons to past studies are available in Appendix $\mathrm{D}$.

While the differing impactor caps affect the magnitude of acceleration, they also affect the duration of impact. With less compliance, impact duration is short, resulting in the rapid acceleration of the head. As observed by Nusholtz et. al, short-duration impacts (less than $15 \mathrm{~ms}$ ) tend to be more injurious to the skull as the loading can exceed the strength of the bone. Due to the increased force on 
the bone, greater compression of the brain was observed in short-duration impacts [22]. In examining Figure 46, the impact duration for Specimen 2842 is approximately $15 \mathrm{~ms}$. Specimen 2871 was subjected to a much shorter duration of approximately $5 \mathrm{~ms}$ due to its low-compliance cap. The effects of impact durations is further discussed in the displacement analysis, as short-duration impacts were seen to cause greater initial compression of the brain tissue.

The coup and contre-coup pressure, measured at and opposite the impact site, are additional means of comparison across data sets. Nahum et al. used these pressures for model validation and identified a linear relationship with resultant linear acceleration, providing a physical explanation of the injury associated with acceleration [41]. Much like acceleration, a broad range of coup pressures has been observed in similarly energetic impacts, ranging from 20 to $180 \mathrm{kPa}$ [22, 41]. In a comparison done by Hardy et al., C241-T5 [25] was compared to Impact-48 performed by Nahum et al. [41], and 76A145 by Stalnaker et al [33]. For C241-T5, a $3.7 \mathrm{~m} / \mathrm{s}$ unhelmeted impact on a rigid anvil, a peak pressure of $153.4 \mathrm{kPa}$ was observed at approximately $4 \mathrm{~ms}$ after impact. Impact-48 and 76A145 reached peak pressures of 123.8 and $140 \mathrm{kPa}$, respectively, for similar input kinematics. With these, and other PMHS observations, impacts tends to follow a predictable time history, occurring in time with peak acceleration, but leading significant head motion [41].

For the present study, only coup pressure was collected for Specimen 2871. The pressure signal shown in Figure 48 shows the short-duration pressure pulse following immediately after a $3.8 \mathrm{~m} / \mathrm{s}$ impact, and rising to $98.4 \mathrm{kPa}$ in $3.75 \mathrm{~ms}$, followed by a pressure drop, reaching $-11.7 \mathrm{kPa}$ at $10.0 \mathrm{~ms}$ after impact. As expected, the timing of these signals lags the acceleration by less than $1 \mathrm{~ms}$. The peak pressures observed in this study are less than those compared earlier [25], likely due to the use of a non-rigid impactor. Despite the lower magnitude, the pressures 
observed in this study follow the expected timing trends and relationship to acceleration (Table 10). A comparison of previous studies and the present study, as well as the pressure-acceleration trends are shown in Figure 50.
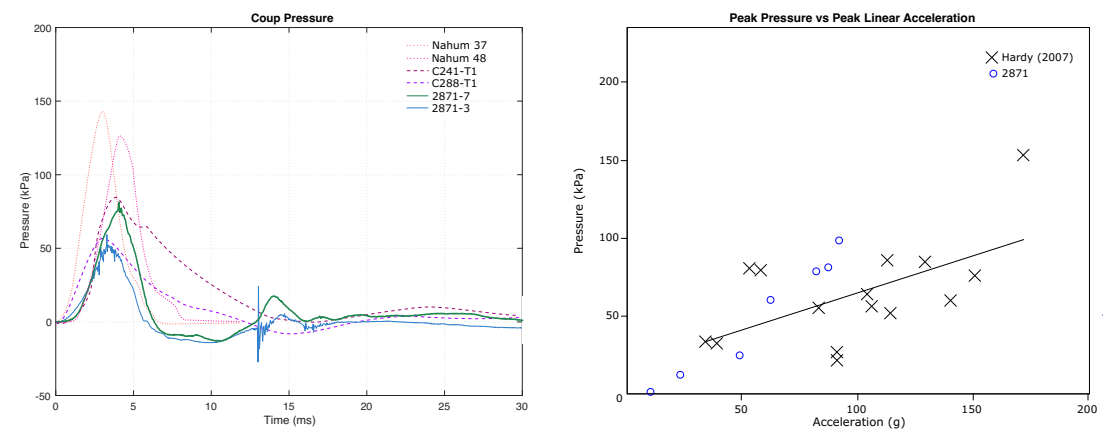

Figure 50: Left: A comparison of pressure signals from previous works, adapted from Hardy (2007) [25] to include signals from 2871-3 and 2871-7. Right: Relationship between pressure and peak linear acceleration, adapted from Hardy(2007) [25] to include data from 2871. The impacts performed in this study fall within the range of impacts performed previously.

The bulk motion of the brain following impact is well established from past studies [24, 25, 35, 36], with three primary characteristics. First, in the regions of the brain observed previously, motion appears to lag that of the skull. Using the skull as a frame of reference, this appears as though the brain is moving towards the impact location. For the earlier discussed trial by Hardy et al., C064-T2, the peak displacement in this phase comes $\approx 20 \mathrm{~ms}$ after impact [25]. Second, as the acceleration of the head peaks, inertial forces in the brain cause it to reverse direction, moving away from the impact site, exceeding the rate of the skull, and stretching to its maximum displacement. In C064-T2, this second phase occurred $\approx 62 \mathrm{~ms}$ after impact [25]. This motion creates the characteristic looping, or figure-8 patterns typically seen throughout the whole brain. The third common characteristic of brain motion is that displacement tends to be more exaggerated near the brainskull boundary. Displacements in these peak regions have been reported between 
9 and $11 \mathrm{~mm}$, and deeper brain displacement is closer to $3 \mathrm{~mm}$ [25].

The motion of the PMHS brains in the present study follow similar trends. Figure 49 presents the smoothed path of two markers, showing the characteristic looping in Specimen 2871. Markers 3 and 19 were chosen for illustrative purposes, however the trend was broadly observed. In particular, the path of Marker 3 clearly shows motion towards the impact site, peaking at a displacement of $5.5 \mathrm{~mm}$ after $18.8 \mathrm{~ms}$ (first phase). This is followed by a reversing motion in the direction of impact, passing its starting position and extending to its peak displacement of $6.5 \mathrm{~mm}$ at $58.8 \mathrm{~ms}$ (second phase). The marker then returns to its starting position. The path of Marker 19 is less clear, though motion towards, and subsequently away from the impact site is still seen, with peak displacement reaching $3.9 \mathrm{~mm}$. The total travel of these markers occurs over approximately 190 ms. Peak displacements for all specimens are shown with the respective time after impact in Table 11. which range from 3.1 to $10.9 \mathrm{~mm}$ in Specimen 2842, and 4.9 to $11.1 \mathrm{~mm}$ in Specimen 2871, each within the range of values expected from previous investigations.

\subsubsection{Comparison of Impacts}

Within the present study, comparisons between displacement responses are helpful to highlight the effects of impact characteristics, repeated impacts, and time between impacts.

The effects of repeated impacts is of concern not just clinically, but also experimentally. As specimens begin to degrade shortly after death, their biofidelity is time-limited, and may also be impact-limited. To evaluate the viability of specimens subjected to repeated impacts, low-speed impacts were performed on each specimen first in order to set a baseline response. Following higher speed impacts, 
a low-speed impact was repeated to compare its response to the baseline. Figures 51 and 52 show the traces for the viable markers in two similar hits, 2842-3, and 2842-7. For 2842-3, displacements are small throughout the entire brain, illustrating minimal distortion. Conversely, in 2842-7, the motion is substantial, especially toward the brain-skull boundary in the frontal region. Between these two impacts were high-speed hits at 3.1, 4.5, and $5.1 \mathrm{~m} / \mathrm{s}$. While these were performed with a high compliance impactor cap, the repeated second-phase displacement may have caused damage at the brain-skull boundary, allowing freedom in these regions. Deeper structures, near the CG, and inferior to the lateral ventricle $(y<20)$, show less freedom of motion, suggesting they may be more resilient in repeated impact scenarios.

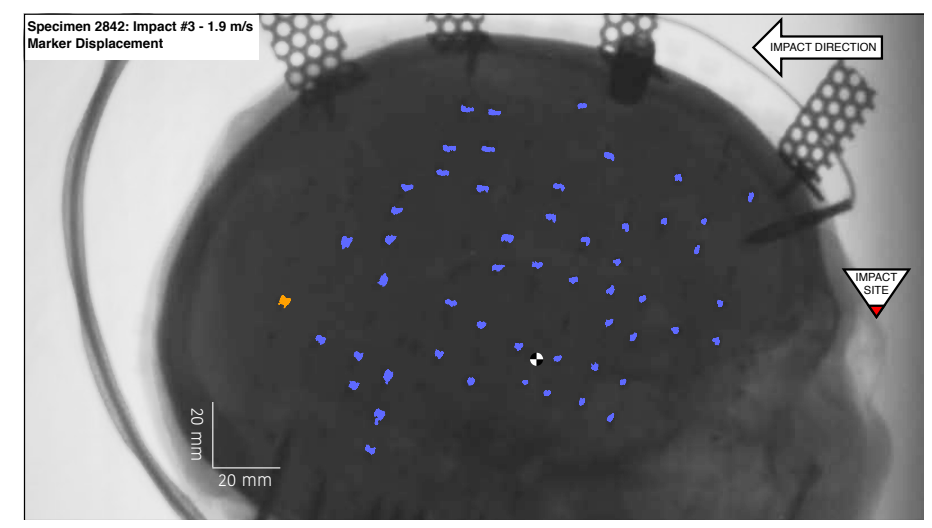

Figure 51: Displacement paths for 2842-3, $1.9 \mathrm{~m} / \mathrm{s}, 0 \mathrm{~ms}<t_{\text {impact }}<180 \mathrm{~ms}$. Increased displacement is seen toward the superior region, and through the cerebellum, while the frontal region and mid-brain exhibit smaller displacements. Peak displacement is highlighted in orange. 


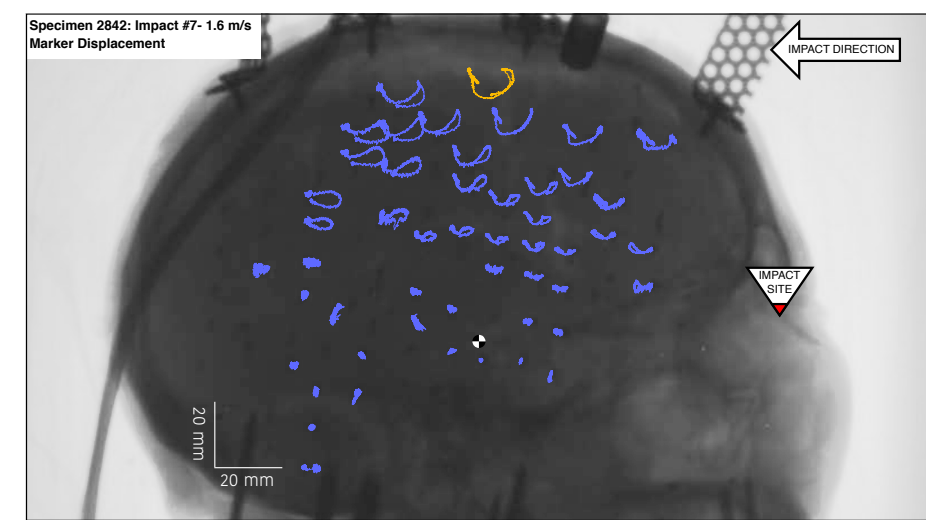

Figure 52: Displacement paths for 2842-7, $1.6 \mathrm{~m} / \mathrm{s}, 0 \mathrm{~ms}<t_{\text {impact }}<180 \mathrm{~ms}$. Notably increased displacement is seen in the superior region, including both frontal and parietal lobes. Though similar impact speed to Impact 3, displacements are more pronounced, indicating some structural changes in the tissue may have occurred. Peak displacement is highlighted in orange.

Comparing the full displacement responses of 2871-1 and 2871-5 (Figures 53 and 54) also informs the effects of repeated impacts. Between these two impacts were three hits at 2.1, 3.0, and $3.1 \mathrm{~m} / \mathrm{s}$ each. While lower than that of the intermediary impacts in 2842, the impact cap was less compliant, increasing the acceleration impulse. Unlike 2842, the later repetition of a low-speed impact did not reveal any significant loss in structure or tissue rigidity in 2871. Low perfusion pressure in 2871-1 may have been the cause of its increased superior frontal and parietal lobe displacements. Perfusion pressure was checked between impacts, and was restored following this impact. The motion in $2871-5$ has a centre of rotation slightly higher than other specimens, as impactor alignment may have shifted slightly. Although the motion paths are not identical, they appear similar in magnitude when observing the entire motion trace. The preservation of this specimen at this stage in the protocol may be more closely related to its lower post-mortem time of $36 \mathrm{~h}$ as compared to $54 \mathrm{~h}$. With the potential for repeated impact damage, interspersed low-speed impacts remain a suitable method for estimating specimen viability and 


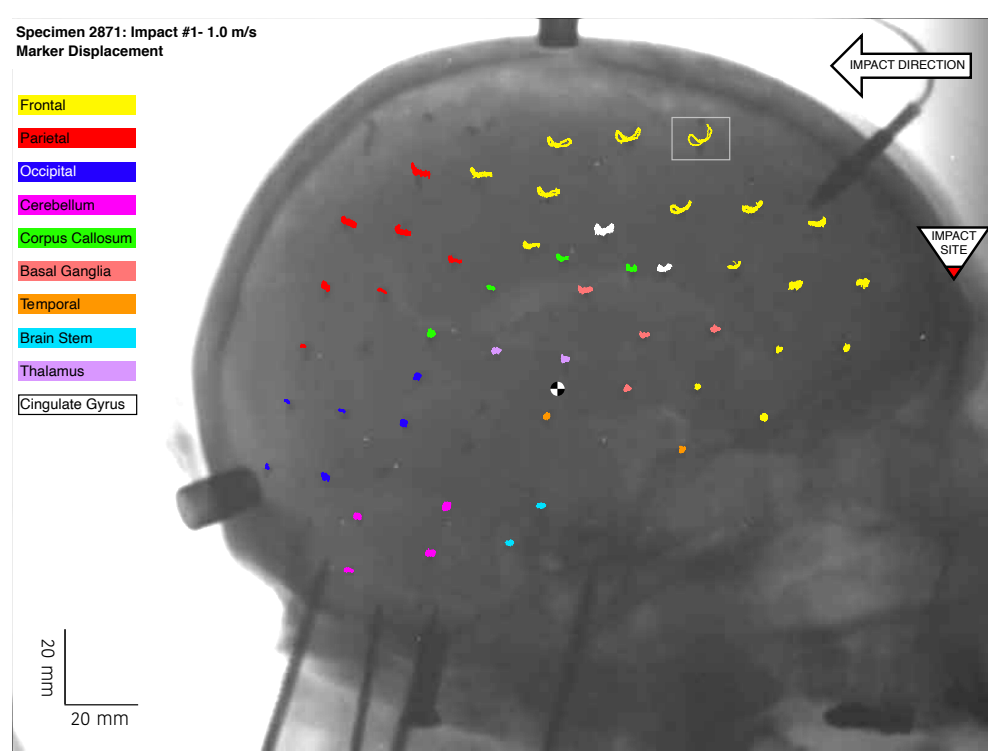

Figure 53: Displacement paths for 2871-1, $1.0 \mathrm{~m} / \mathrm{s}, 0 \mathrm{~ms}<t_{\text {impact }}<180 \mathrm{~ms}$. Regional colour coding per post-experiment MRI. Exaggerated frontal-superior displacements may be indicative of a loss of perfusion pressure.

should remain part of testing protocols.

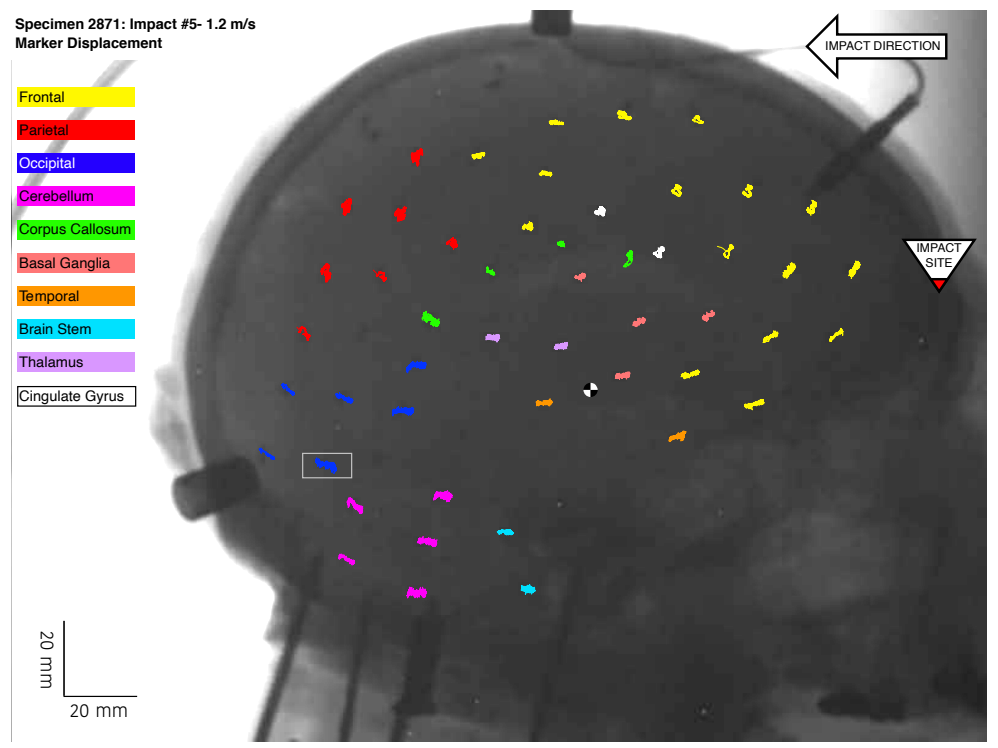

Figure 54: Displacement paths for 2871-5, $1.2 \mathrm{~m} / \mathrm{s}, 0 \mathrm{~ms}<t_{\text {impact }}<180 \mathrm{~ms}$. Regional colour coding per post-experiment MRI. This impact was performed to check the low-speed response of the specimen following repeated higherspeed tests. Relatively small displacements $(<5 \mathrm{~mm})$ and organized patterns suggest the specimen remains intact. 
The effect of impactor cap compliance and impact duration have been discussed in terms of kinematics and pressure. These impact characteristics also have a visible effect on whole-brain displacement when comparing marker traces. Figures 55 and 56 show displacement traces for 2842-4 and 2871-3, respectively. Each of these impacts was performed at approximately $3 \mathrm{~m} / \mathrm{s}$. The stiffer impactor used with 2871 yields larger displacements, with motion paths that are more organized. These traces show a clear swirl pattern around the centre of rotation, slightly superior of the CG of the head. The looping patterns in 2871 are present, though a greater degree of impact-direction displacement is seen, flattening the loops by comparison to 2842. While these differences are expected and well established in literature, the increased dispersion of markers through the brain provides greater understanding of how these kinematic changes propagate through the structures of the brain.

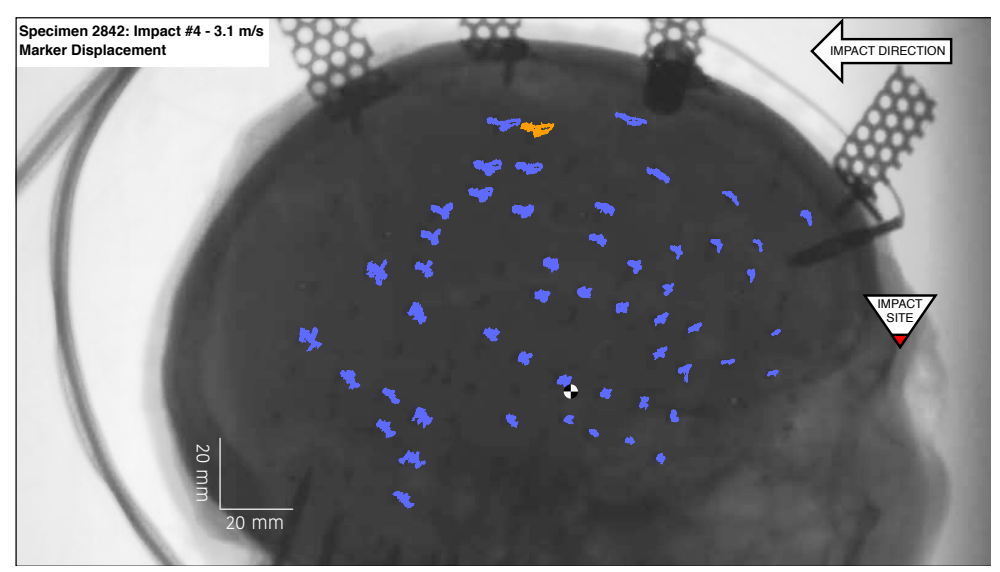

Figure 55: Displacement paths for $2842-4,3.1 \mathrm{~m} / \mathrm{s}, 0 \mathrm{~ms}<t_{\text {impact }}<180 \mathrm{~ms}$. Variations in displacement magnitude are similar to Impact 3, though more clearly defined looping patterns can be seen. Peak displacement is highlighted in orange. 


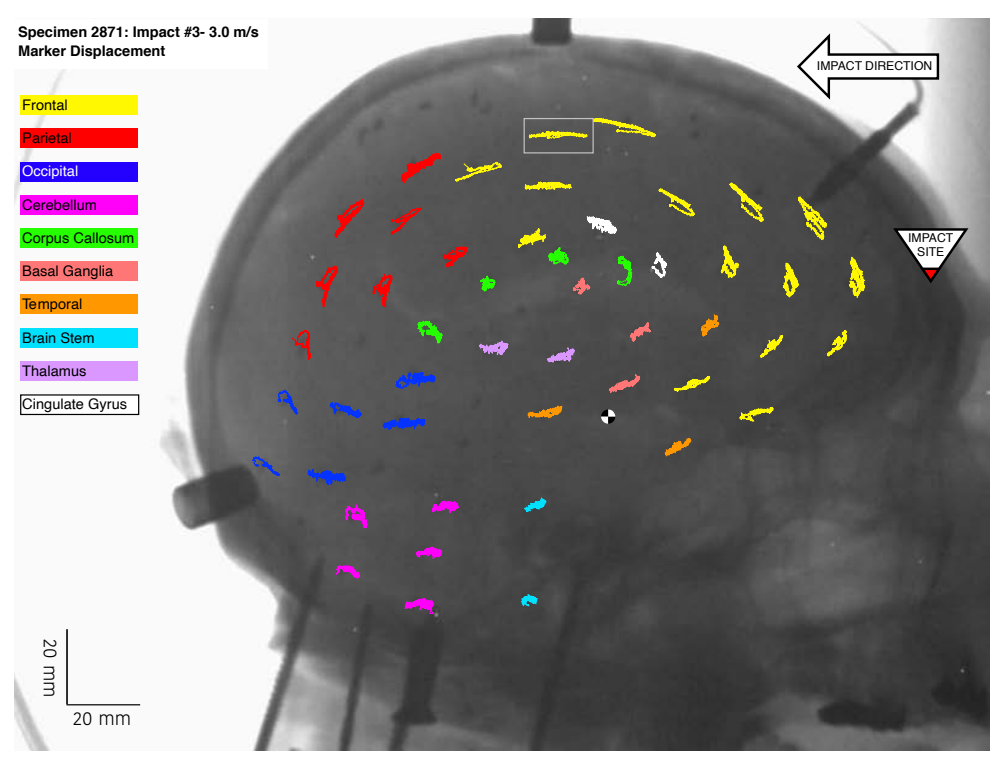

Figure 56: Displacement paths for 2871-3, $3.0 \mathrm{~m} / \mathrm{s}, 0 \mathrm{~ms}<t_{\text {impact }}<180 \mathrm{~ms}$. Regional colour coding per post-experiment MRI. Paths are similar to Impact 2, though with increased magnitude, and a clearer centre of rotation.

Repeated impacts of the same intensity were performed consecutively for 28716 and 2871-7. Though differing slightly in impact speed and resultant kinematic and pressure responses, these impacts elicited similar displacement traces and peak displacements in the frontal and parietal regions, as shown in Figures 57 and 58. The occipital region and cerebellum exhibited shorter traces in 2871-7. Unlike previous impacts with this specimen, in 2871-7, the first phase displacement was less than that of the second phase $(10.2 \mathrm{~mm}$ and $11.1 \mathrm{~mm}$ respectively). These displacements are similar in magnitude to $2871-6$, but the shift of peak displacement from first to second phase may be indicative of tissue deterioration, or relaxation. As discussed previously, repeated high-intensity impacts may reduce the fidelity of specimens beyond the first five impacts. Considering the similar responses in the frontal and parietal regions, this finding underscores the repeatability of the impacting method. With respect to the posterior structures, this finding also highlights the benefits of broad dispersion of contrast markers, as subtle 
regional differences can be detected.

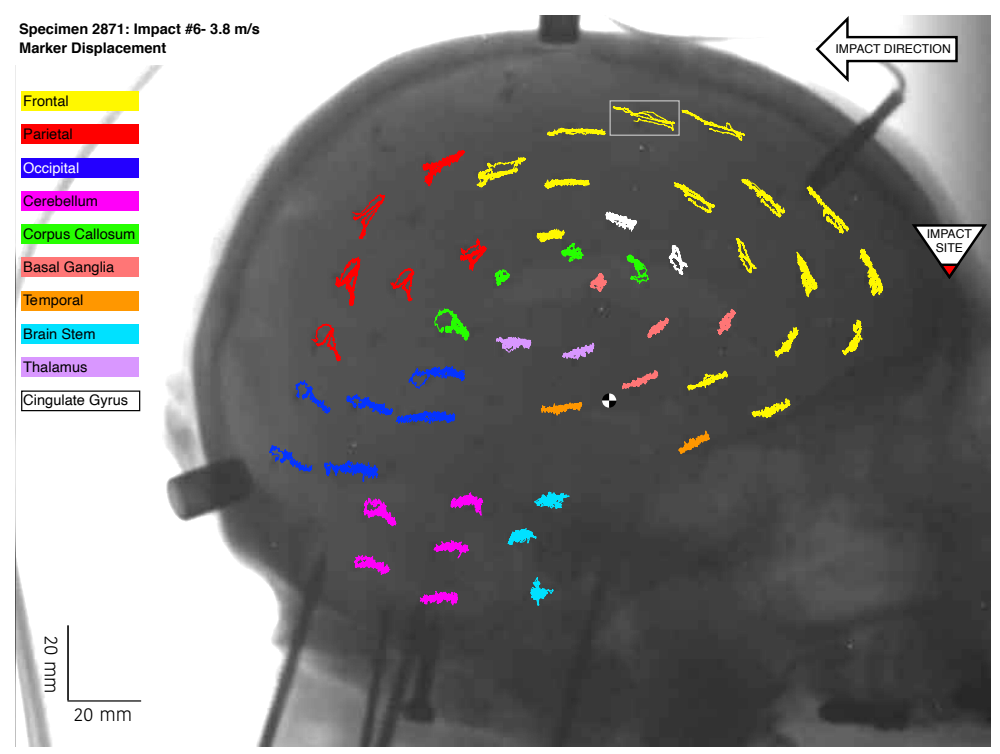

Figure 57: Displacement paths for $2871-6,3.8 \mathrm{~m} / \mathrm{s}, 0 \mathrm{~ms}<t_{\text {impact }}<180 \mathrm{~ms}$. Regional colour coding per post-experiment MRI. The response is similar to that of previous impacts, with an overall increase in magnitude, due to the increase in impact speed. The posterior region $(-60<x<30 \mathrm{~mm}, 0<y<40 \mathrm{~mm})$ experiences greater $y$-direction motion, possibly due to ventricular motion. 


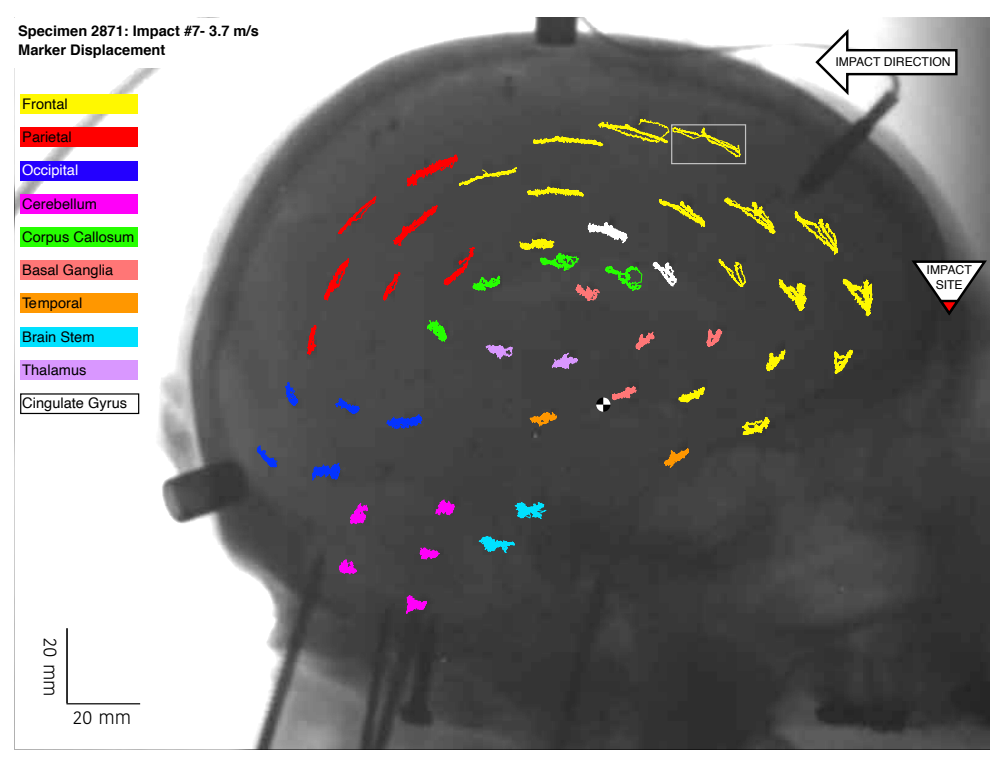

Figure 58: Displacement paths for 2871-7, $3.7 \mathrm{~m} / \mathrm{s}, 0 \mathrm{~ms}<t_{\text {impact }}<180 \mathrm{~ms}$. Regional colour coding per post-experiment MRI. Similar impact characteristics as Impact 6, and subsequently a similar response. Motion in the y-direction is less pronounced near the posterior periventricular region than in previous impacts. 


\subsubsection{Regional Displacements in the PMHS Brain}

The PMHS impacts performed in this study have yielded similar results to past studies in terms of kinematic response, intracranial pressure, and whole-brain motion characteristics. In comparing impacts from the present study with each other, variations in impact responses can clearly be identified with respect to impact speed and duration. The aim of this study, however, goes beyond recreating past results or evaluating impact characteristics; its focus is on the measurement of displacement of the brain within targeted structures and regions. The combined use of MRI imaging for marker placement planning, rigorous marker insertion techniques, HSXR imaging capable of high spatial and temporal resolution, and purpose-built tracking code allow a more in-depth review of the impact response of brain structures.

The marker placement for each specimen was guided by the use of preexperiment MRI images. For Specimen 2842, MRI helped to identify the limits of the cerebrum and the position of the cerebellum. While this was helpful, advanced targeting of smaller regions was not possible at the time. For Specimen 2871, the resultant MRI images provided greater measurement details, permitting targeted marker placement. The corpus callosum and brainstem were targeted, with 5 of 6 inserted markers being embedded within their targeted region; in the corpus callosum, Markers 49-51, and in the brainstem, Markers 52 and 53. It was expected that remaining markers inserted through the parenchyma would fall within frontal, parietal, temporal, and occipital lobes, as well as the cerebellum. Following the post-experiment MRI, additional small structures containing markers were identified by the neurosurgeon project partners, including an extra unexpected marker in the posterior region of the corpus callosum (Marker 33). These number of markers in each region is shown in Table 12, and regions are highlighted 
Table 12: Marker count per region - Specimen 2871

\begin{tabular}{cc} 
Region & Marker Count \\
\hline Frontal Lobe & 16 \\
Parietal Lobe & 7 \\
Occipital Lobe & 6 \\
Cerebellum & 5 \\
Corpus Callosum & 4 \\
Basal Ganglia & 4 \\
Cingulate Gyrus & 2 \\
Thalamus & 2 \\
Temporal Lobe & 2 \\
Brain Stem & 2 \\
\hline
\end{tabular}

on the displacement traces discussed earlier (Figures 51,58). While it is expected that similar localization of markers will be possible for Specimen 2842, this work is presently pending. As such, this discussion will focus on regional results from Specimen 2871.

In Figures 5965, regional groups of markers have been separated into timeresolved displacement plots. Data shown in these plots was smoothed using a Savitzky-Golay filter (window of 15 points) [48]. This particular filter was chosen for its ability to provide noise reduction without significant loss of high-frequency signal content (peak displacements). The displacements shown are the resultant horizontal and vertical displacement, starting at the moment of impact. As bulk head motion was removed in the tracking process, displacements are the frame of reference of the skull. In these figures, each distinct structure exhibits its own complex motion, revealing the heterogeneous nature of the brain. A complete set of time-resolved regional resultant displacements for 2871-1 through 2871-7 are provided in Appendix $\mathrm{D}$. Individual marker traces are distinguished with different coloured lines. 
For the first, and lowest intensity impact, the time-resolved regional displacements in the frontal, parietal and occipital lobes, and cerebellum are shown in Figure 59. In the frontal lobe, an early impact response is seen in about half of the markers, peaking after at approximately $20 \mathrm{~ms}$ and $4 \mathrm{~mm}$. These markers displace in the opposite direction $40 \mathrm{~ms}$ after impact, reaching a second phase displacement of $3.5 \mathrm{~mm}$ at $\approx 60 \mathrm{~ms}$. The markers in the parietal region show a similar early rise at $20 \mathrm{~ms}$, however upon reversing direction, they do not overshoot their initial position. In the occipital lobe and cerebellum, gradual increases occur at about $50 \mathrm{~ms}$ after impact, but the initial displacement observed in the superior regions is not present. These inferior structures tend to move together at this impact speed, implying they may be stiffer, or more homogeneous. In the deeper structures, shown in Figure 60, the cingulate gyrus and basal ganglia, which are close to the frontal lobe, exhibit a similar response. The displacements are reduced overall, as these structures are further from the brain-skull boundary. The markers in each of the thalamus and corpus callosum move together for the first phase, but disassociate after about $40 \mathrm{~ms}$. While the gradient of deformation across the brain is not particularly intense for this low-speed impact, the presence regional displacement variations is indicative of tissue strain.

Examining the effect of impact speed on the regional displacements, Figures 61 and 62 show the time-resolved displacements for the same regions for 2871-3. The response shape similar to that of the first impact, though peak displacements are uniformly higher. In the frontal region, markers achieve displacements between 4 and $9 \mathrm{~mm}$ at $\approx 20 \mathrm{~ms}$. Markers then reverse direction, looping past the origin at approx $40 \mathrm{~ms}$, and reaching their second phase peak $(60 \mathrm{~ms})$. The timing of these phases is consistent with the literature [25]. Comparing this to the less-intense first impact, marker phases occur with similar timing, but the increase in displacement 


\section{Specimen 2871 - Impact $1-1.0 \mathrm{~m} / \mathrm{s}$ Regional Displacement}
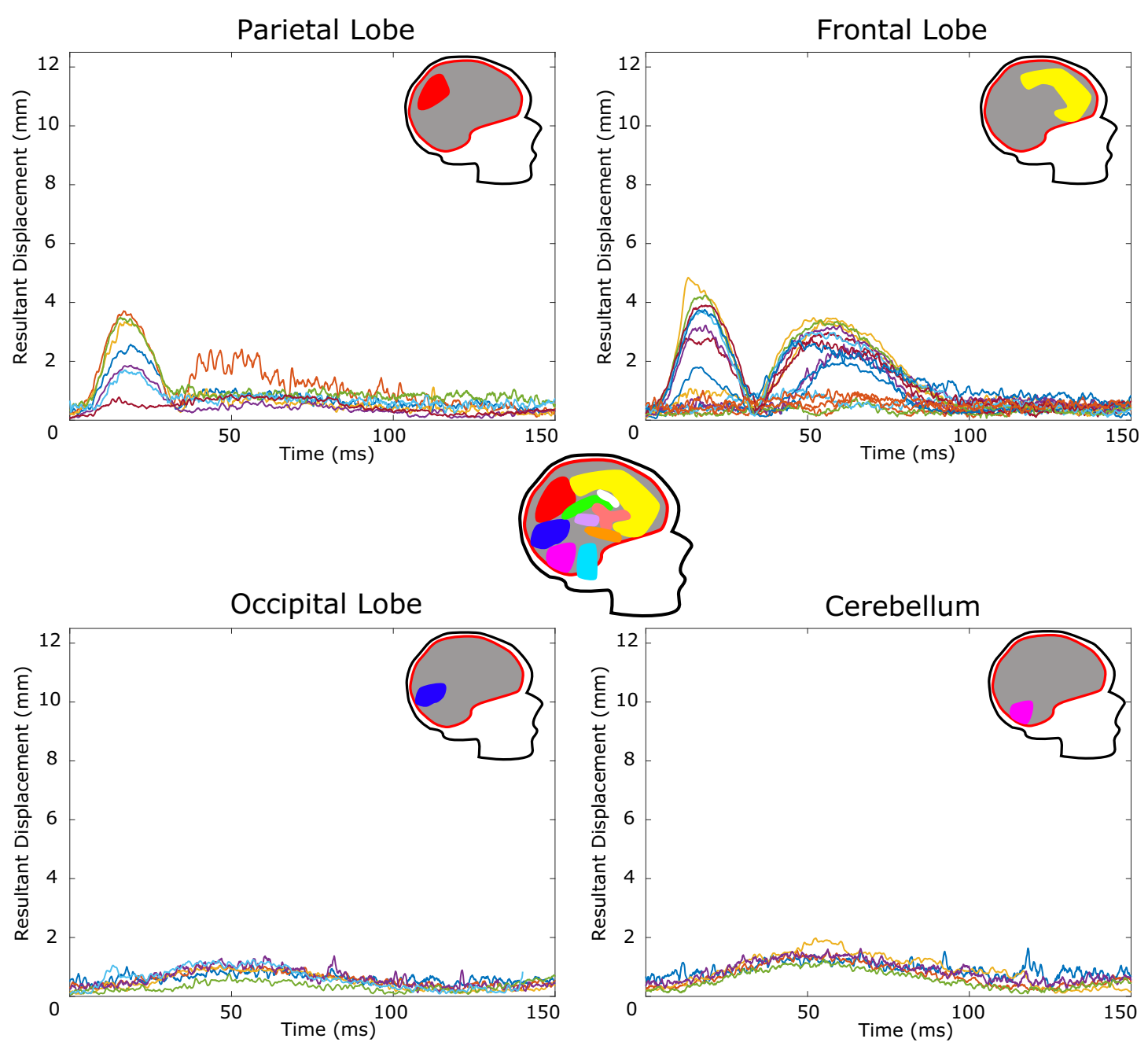

Figure 59: Regional displacements observed in the parietal, frontal, and occipital lobes, as well as the cerebellum, for 2871-1. Displacements are observed to vary across the different regions.

drives up the tissue velocity, which is likely to increase rates of strain across the brain and within regions. For some frontal lobe markers, an additional third phase is seen $(120 \mathrm{~ms})$, with peaks of $6 \mathrm{~mm}$, likely coinciding with the specimen reconnecting with the impactor. Following this phase, the tissue appears to gradually settle toward its original position after $150 \mathrm{~ms}$. 
Specimen 2871 - Impact $1-1.0 \mathrm{~m} / \mathrm{s}$

Regional Displacement
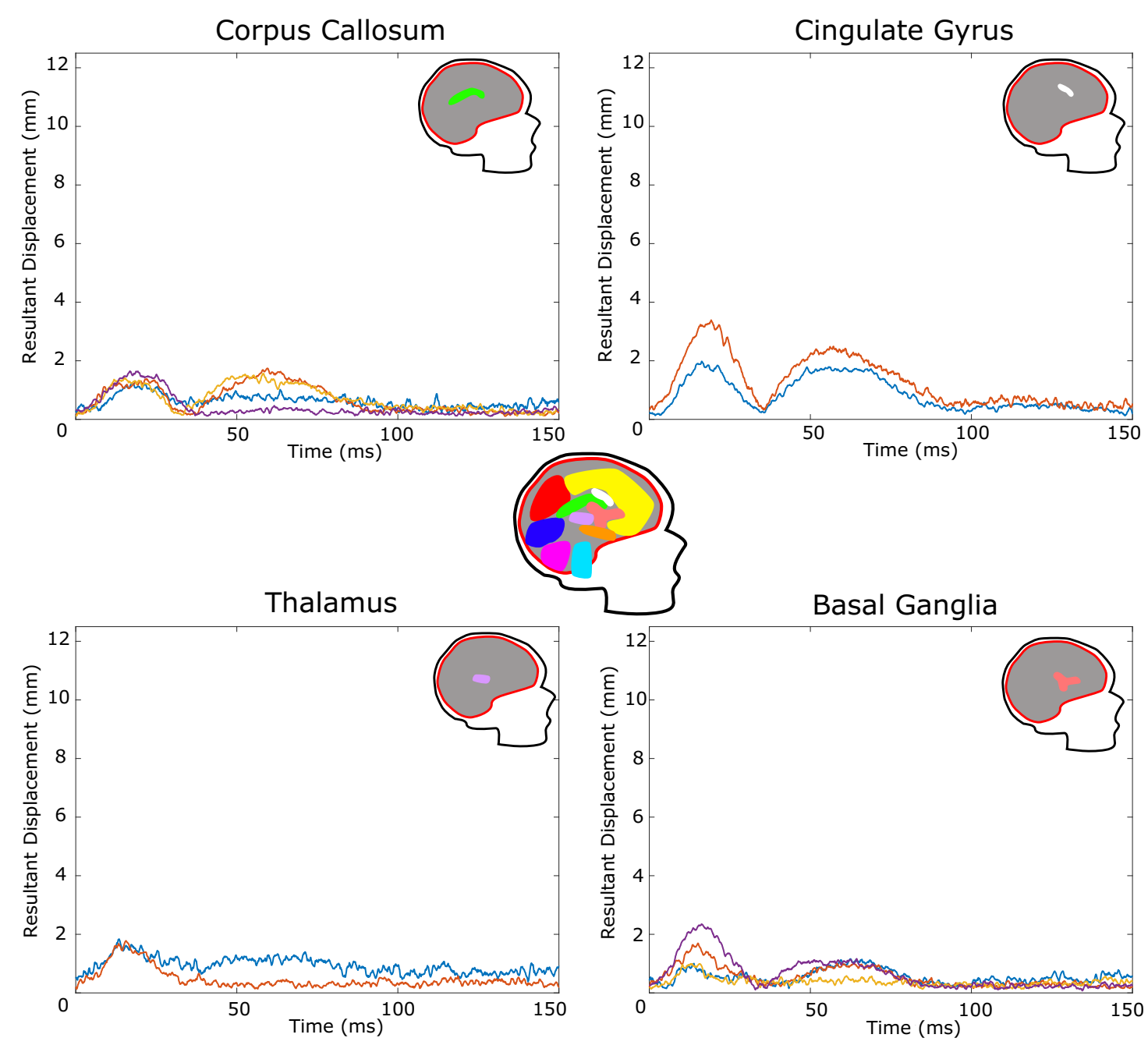

Figure 60: Regional displacements observed in the corpus callosum, basal ganglia, thalamus, and cingulate gyrus for 2871-1. Displacements vary from region to region, highlighting the heterogeneous nature of the tissues.

The parietal region shows a more locally homogeneous response, though there is a range of displacement magnitudes seen, which may correlate with depth. Similar to the first impact, the initial response matches that of the frontal lobe, followed by a long-duration drift back toward the origin. A third phase $(120 \mathrm{~ms})$ interrupts the gradual drift, pushing markers to displacements between 4 and $6 \mathrm{~mm}$, as the 


\section{Specimen 2871 - Impact $3-3.0 \mathrm{~m} / \mathrm{s}$ \\ Regional Displacement}
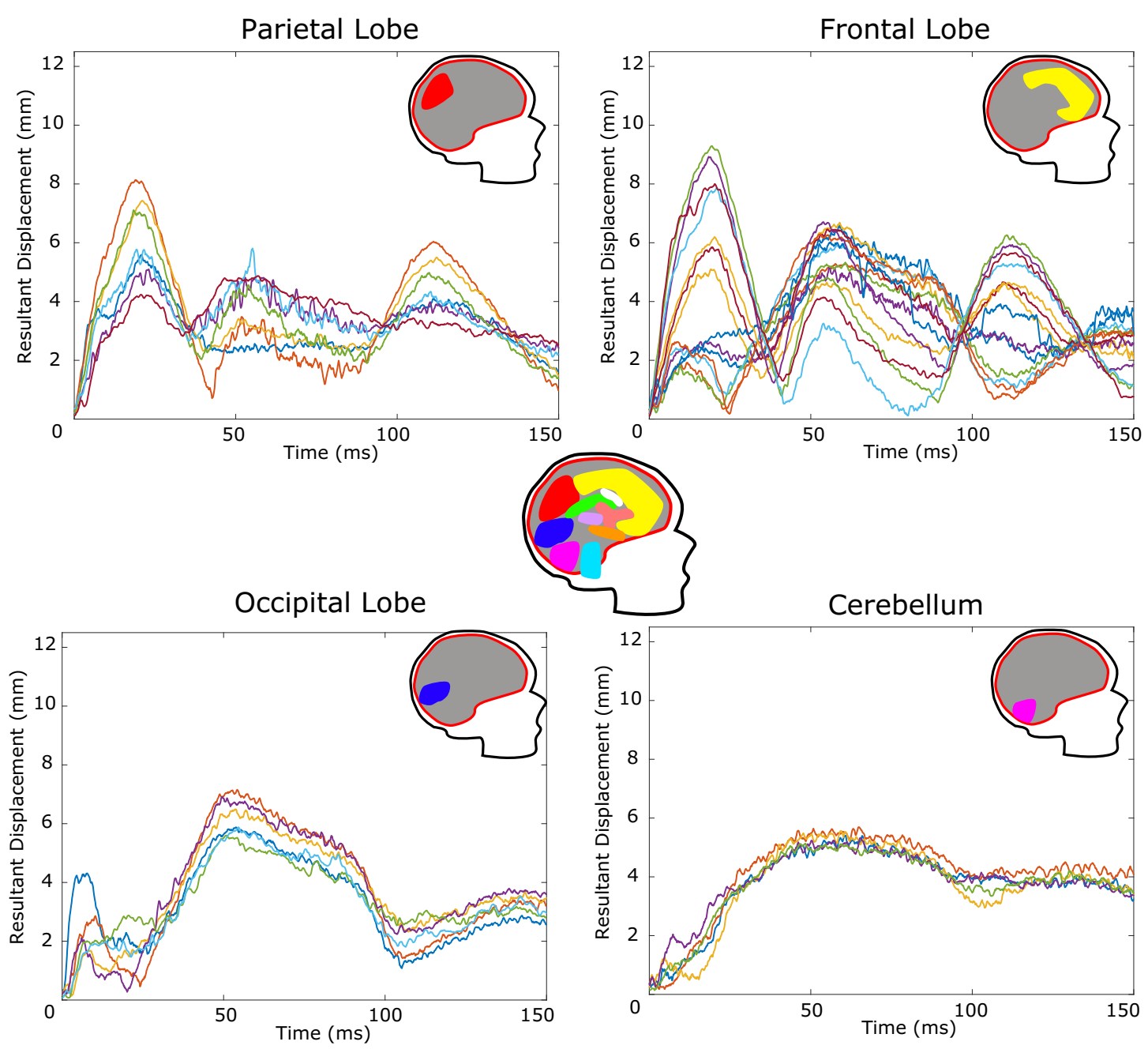

Figure 61: Regional displacements observed in the parietal, frontal, and occipital lobes, as well as the cerebellum, for 2871-3.

specimen rebounds back into the impactor ram. The occipital lobe and cerebellum again exhibit more rigid responses, with markers deviating little from each other. As these structures appear to move as a whole, the connective tissues between neighbouring structures may be more susceptible to strain. A single occipital marker exhibits a sharp displacement of $4 \mathrm{~mm}$ earlier than the others, approximately $5 \mathrm{~ms}$ after impact. This occipital marker may coincide with the posterior 
Specimen 2871 - Impact $3-3.0 \mathrm{~m} / \mathrm{s}$

Regional Displacement
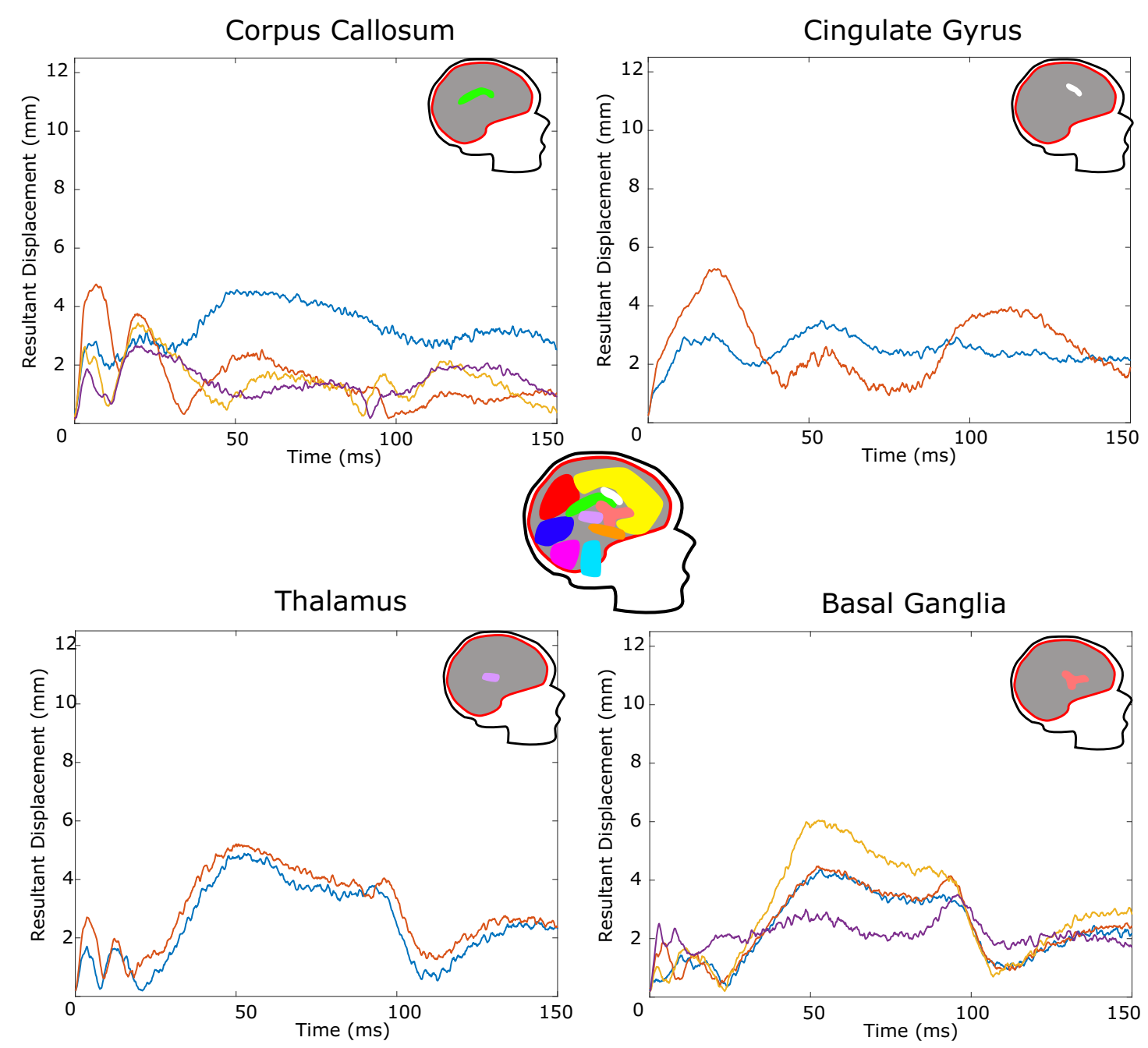

Figure 62: Regional displacements observed in the corpus callosum, basal ganglia, thalamus, and cingulate gyrus for 2871-3.

portion of the corpus callosum which shows a similar trend in all of its markers. The corpus callosum motion may be the result of fluid motion inside the lateral ventricle. The nearby thalamus and basal ganglia also show a sharp rise in the first $5 \mathrm{~ms}$, though to a lesser peak of about $2 \mathrm{~mm}$. The thalamus markers move together in a synchronised way, suggesting less internal deformation, while the 
basal ganglia exhibits a breadth of displacements ranging from 2 to $6 \mathrm{~mm}$ at approximately $50 \mathrm{~ms}$ after impact, approximately in time with the second phase in the nearby frontal lobe. The cingulate gyrus shows two distinct paths. Comparing these paths to those in the adjacent frontal lobe, each shows similarities to the two different frontal lobe responses.

Closer examination of the frontal lobe shows two distinct responses, one with early peak displacements, occuring $20 \mathrm{~ms}$ after impact, and one with peaks at $60 \mathrm{~ms}$. Dividing the region further, it is clear that the inferior, prefrontal regions exhibit a different response to that of the superior frontal cortex, as showin in Figure 63. These responses show that the inferior regions of the frontal lobe may act more uniformly, whereas the superior region exhibits a greater range of displacement. The range of displacement in the superior region may be due to uniform rotation about a single point, which does not appear to occur in the inferior region. The difference in response between the two regions suggests that strain may develop between these two distinct zones. 
Specimen 2871 - Impact $3-3.0 \mathrm{~m} / \mathrm{s}$

Frontal Lobe Displacement
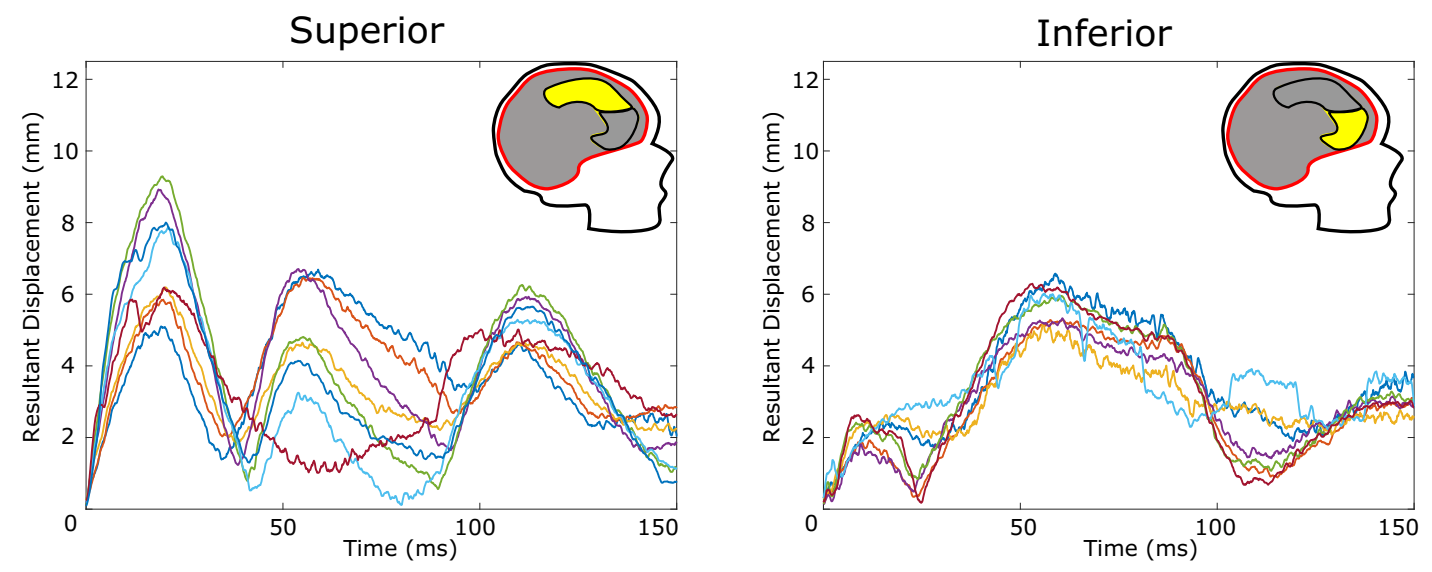

Figure 63: Displacements in the frontal lobe are broken into superior and inferior regions for 2871-3. A distinct difference in motion is observed in each of these regions, despite being part of a larger functional structure. This phenomenon is observed across all impacts.

Previously, 2871-6 and 2871-7 were compared by their whole-brain response. That comparison revealed that while the motions through much of the brain appeared similar, the occipital lobe and cerebellum had distinctly reduced traces. Further examination of these regional displacements is shown in Figures 64 and 65 Between these two impacts, the initial displacements occurring before $15 \mathrm{~ms}$ appear to be similar. Following this, 2871-6, the occipital lobe exhibits a strong reversing displacement approximately $25 \mathrm{~ms}$ after impact, exceeding what was seen in the lower, but similar impact, 2871-3. The subsequent hit, 2871-7, by comparison exhibits a very different response in the occipital lobe, with almost no additional displacement observed until $\approx 110 \mathrm{~ms}$ after impact, when the specimen bumps back into the impactor. Given this response, it may be possible that the intense displacement during 2871-6 resulted in damage to the region, reducing its impact response.

Similar to the occipital lobe, the displacement through the cerebellum is similar 
$2871-6-3.8 \mathrm{~m} / \mathrm{s}$
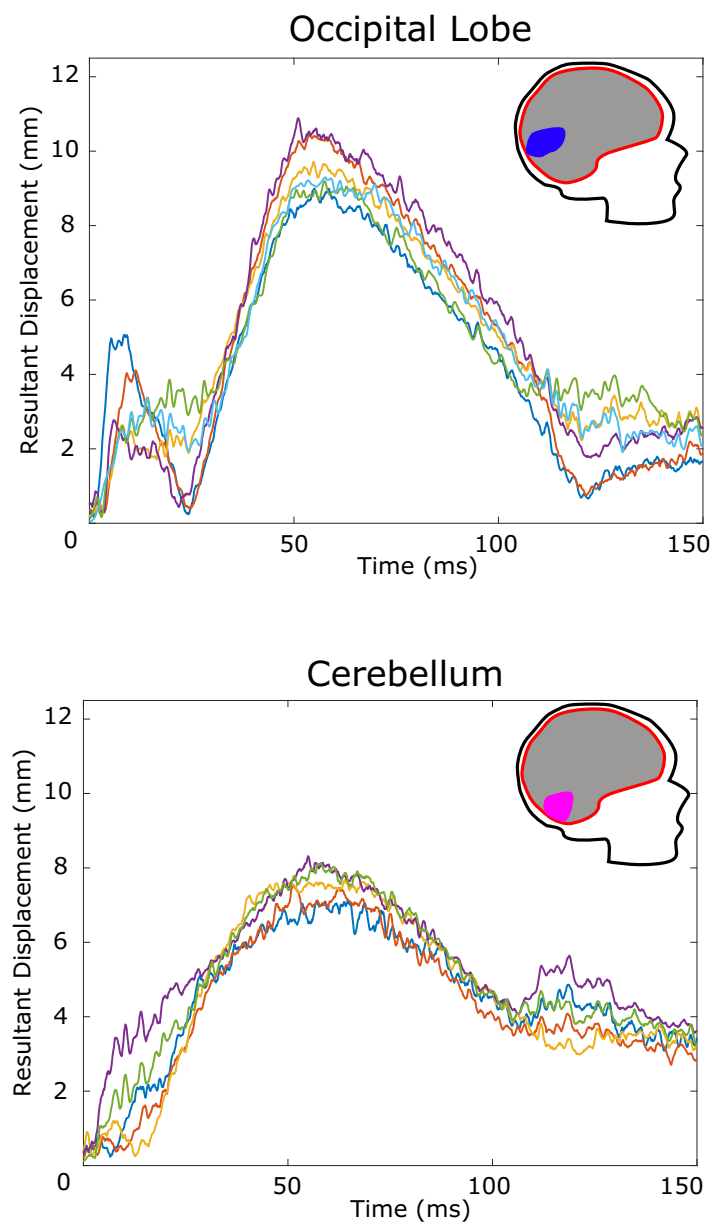

$2871-7-3.7 \mathrm{~m} / \mathrm{s}$
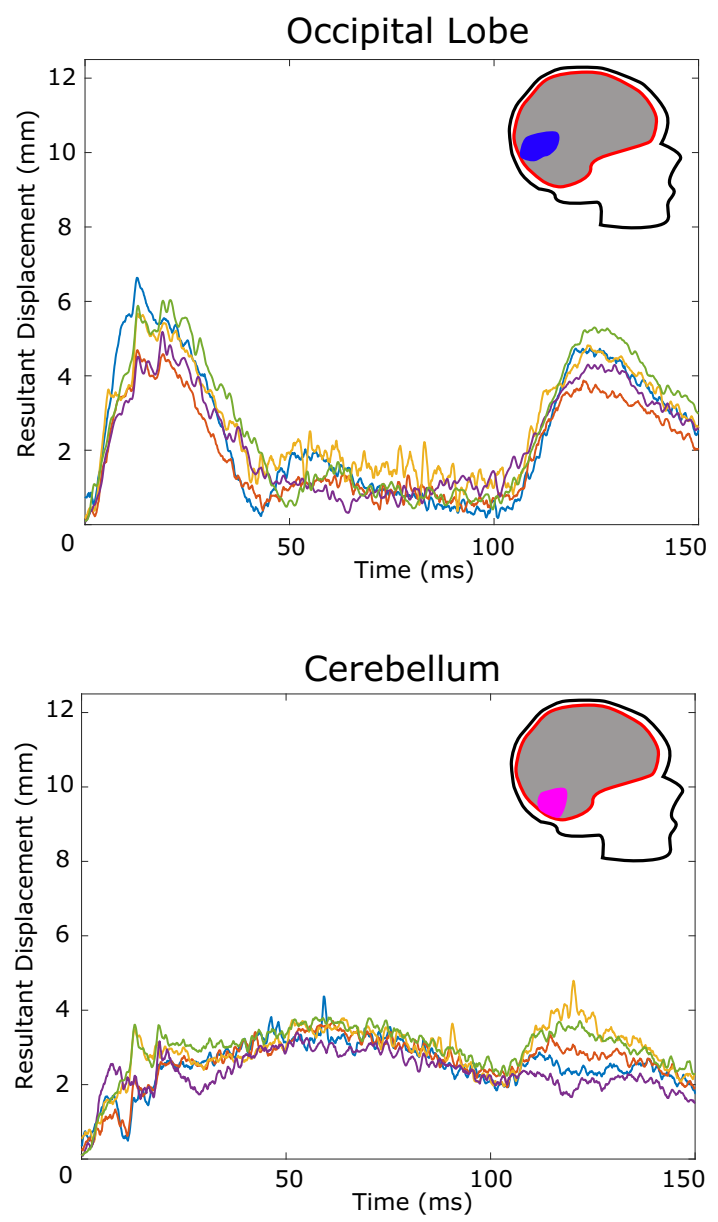

Figure 64: Varying local displacements patterns are observed between two similar impacts, 2871-6 and 2871-7, within the occipital lobe and cerebellum. The response observed in 2871-6 is similar to other impacts. This changing response may be indicative of damage to the region, altering the response in the later impact.

in 2871-6 to 2871-3, though expectedly higher magnitude. The markers in the cerebellum continue to move mostly together, signalling it has not lost any stiffness. In 2871-7, while the homogeneity remains, the overall response is reduced, even when compared to the lesser 2871-3. Like the occipital lobe, the cerebellum may have experienced damage in 2871-6, reducing its ability to move during impact.

Additional differences in response for these similar impact configurations were 
$2871-6-3.8 \mathrm{~m} / \mathrm{s}$

Thalamus

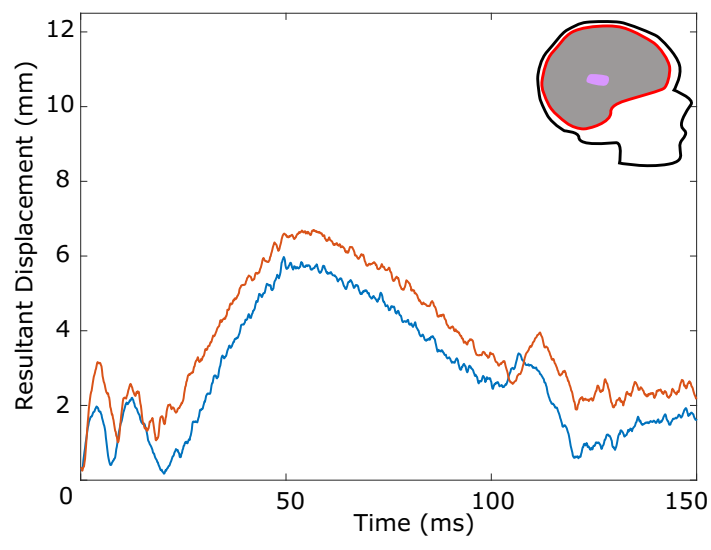

Basal Ganglia

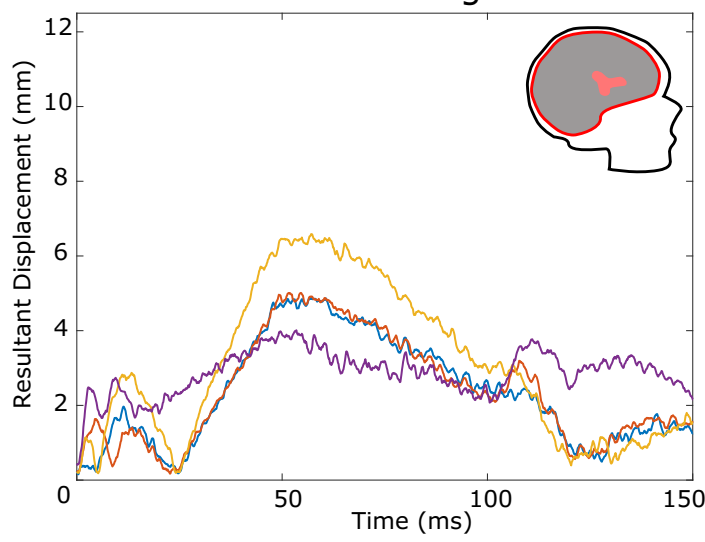

$2871-7-3.7 \mathrm{~m} / \mathrm{s}$

Thalamus

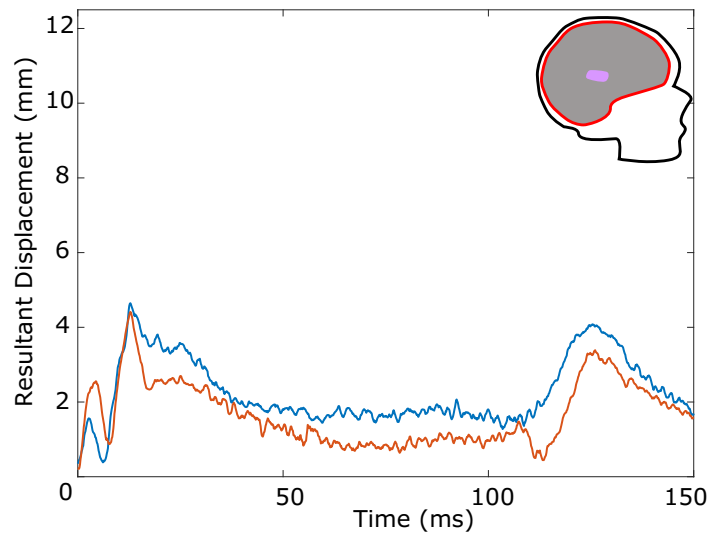

Basal Ganglia

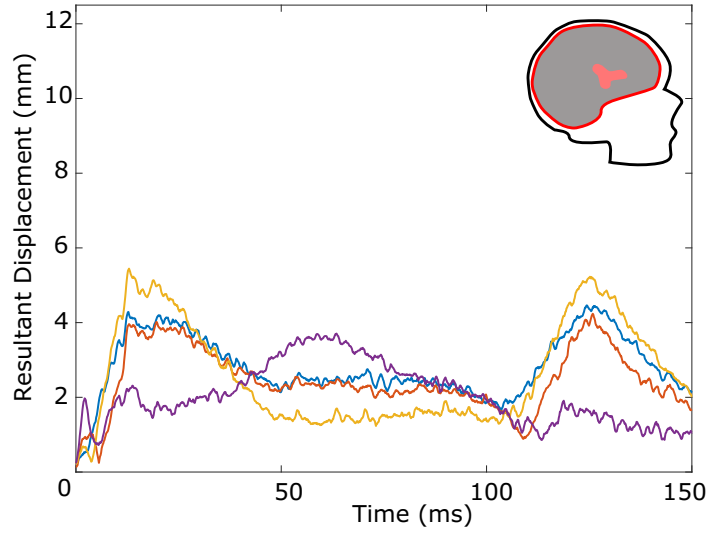

Figure 65: Varying local displacements patterns are observed between two similar impacts, 2871-6 and 2871-7, within the thalamus and nearby basal ganglia. The response observed in 2871-6 is similar to other impacts. This changing response may be indicative of damage to the region, altering the response in the later impact.

seen in the thalamus and basal ganglia. Much like the occipital lobe, the response of the thalamus in 2871-6 occurs quickly, followed by a reversal and peak displacement of $6 \mathrm{~mm}$ at $60 \mathrm{~ms}$. This response is similar to the thalamus response in 28713 , as with other regions. In 2871-7, however, this response is markedly different, with an initial displacement, followed by very little movement, until the specimen bumps back into the impactor at $\approx 110 \mathrm{~ms}$. The nearby basal ganglia responds as expected in 2871-6, with minimal displacement early on, followed by a peak at 
$60 \mathrm{~ms}$, and a range of displacements from 4 to $6 \mathrm{~mm}$. The response of the basal ganglia in the final impact exhibits early motion, much like that of the thalamus, with little variation across the structure, unlike its response in previous impacts.

While at first glance, 2871-6 and 2871-7 appeared to be similar, deeper investigation of their regional responses has revealed that they differ across multiple regions. These differences are likely due to damage caused by the repeated impacts performed on Specimen 2871, which may be confirmed in a later dissection of the specimen. This finding is pertinent to future data collection, as repeated impacts should be performed with care, as to ensure that the data collected is representative of biofidelic PMHS tissue. 
From the results discussed in this chapter, a few key points have emerged. Resolving the motion in small structures and identifying their inherit differences reveals the heterogeneity of the brain and further underscores the need for regionally targeted displacement studies. While this study has examined only a single series of PMHS impacts for regional displacements, it has shown that this methodology can be used moving forward to extract more data from these challenging experiments. The long-term viability of specimens is of interest; repeated impacts may cause structural changes in the tissue, and as such, skew results collected in later trials. Given this, low-speed viability checks should be performed when possible. In addition, the incorporation of varying impact characteristics is also necessary to assess the response of the brain across a broad parameter space. Deeper analysis of the regional and structural differences in response of the brain to impact may be the key to future strain model development and validation. 


\section{Chapter 7}

\section{Conclusion}

This document describes experimental work performed in order to support deeper understanding and mitigation of traumatic brain injuries caused by impacts to the head. Clinically, damage in discrete regions of the brain, notably the corpus callosum and brainstem, is of great concern, yet experiments showing displacement or deformation in these regions have not yet been feasible. The models and tools presently used for the development and testing of protective equipment, including helmets, are based on limited empirical data which lacks clarity in these regions. While finite element models are used to predict injurious levels of strain in different regions of the brain, the validation data from cadaver models has not achieved a level of precision capable of confirming simulation predictions. This thesis presents an improved biofidelic PMHS methodology, incorporating the direct degassing of the ventricular system, upright impact orientation, and use of advanced HSXR and purpose-built image processing and particle tracking. These advances to the study of TBI and its root causes can be used in future PMHS impact studies, and will help to further determine how and why the brain deforms during impact. This work will support the collection of data needed to validate and calibrate the next generation of finite element models and head impact simulations, and provide insight to clinicians on the mechanical causes of TBI. A final summary 
of the goals of this thesis, along with methodology and technical recommendations follows below.

\section{Validating PMHS studies}

A comprehensive review of past studies revealed that across a broad range of impact characteristics, resultant head kinematics, intracranial pressure, and wholebrain displacement measurements tend to fall within an expected, though broad, range of values. Early observations characterized the qualitative trends typical of brain, with more recent studies focusing on the use of cadaver models. In reviewing these past works, attention was paid to the experimental methods and limitations, informing the methods and advances developed for the present PMHS study. The broad results space served as a means of validating the results obtained from the study.

\section{Developing measurement techniques and test methodology}

To achieve the improvements to temporal and spatial resolution necessary for this study, the Carleton University HSXR laboratory was used. To address past concerns relating to the dynamic coupling of embedded contrast markers in PMHS brains, a Taguchi-based sensitivity study was performed. The study evaluated how marker design parameters affect impact displacements in tissue-simulating gel. Density, stiffness, and marker spacing were varied across nine new marker designs. Additional markers (NDTs) replicating those used in past studies were also tested. The motion observed in drop tests was compared to that of a neat material, embedded with ink-based massless markers. The results of this study suggest that the sensitivity of marker parameters shifts through the phases of motion. In the first phase of motion after impact, density is the most sensitive parameter, while stiffness and spacing become more prevalent in the second phase. This study helped to identify an elastomeric, low-density, high contrast particle for use in the PMHS study. 
Test methodology for the PMHS specimens was primarily developed through the review of past studies. The use of MRI imaging to plan the insertion of between 60 and 80 tissue embedded contrast markers permitted targeting discrete regions of the brain. Direct filling of ventricular space for the removal of intracranial gases and an upright impacting position were performed in order to improve biofideltiy of PMHS specimens.

\section{Observing brain motion}

Two fresh, unfrozen, unembalmed PMHS head specimens were subjected to seven impacts each, with varying impact characteristics. Kinematic, pressure, and displacement measurements were validated against expected values from past studies. Longer-duration impacts reduced relative motion in the first-phase after impact. Specimens appeared to remain in-tact for up to 6 repeated impacts, informing the need for careful monitoring of specimen viability.

The observation of previously unreported brain motion was possible due to the high spatial and temporal resolution of the $\mathrm{X}$-ray system, combined with the intricate planning and placement of embedded markers. Discrete regions and structures in the brain were observed to deform independently of each other. The cerebrum and cerebral cortex were observed to experience greater displacements due to impact than deeper structures, with peak displacements occurring in these regions. The frontal lobe exhibited two distinct motion profiles; one corresponding to the inferior region, and one to the superior region. The cerebellum and occipital lobes were not observed to deform internally, but their motion was distinct from other regions. The onset of rapid motion, coincident with the moment of impact, was seen in the corpus callosum, thalamus, and basal ganglia. These inter- and intra-regional displacement modes highlight the need for refined modelling data. 


\section{Recommendations and Future Work}

As the work in this field continues at Carleton University, some recommendations to improve upon the quality, and further validate results can be made. Pressurization of PMHS heads is challenging and has a significant effect on brain motion due to impact. As such, repeated pressurization checks and recirculation of lost fluid would be beneficial in future studies. Additionally, it may be necessary to further validate the methods used to fill and degas the ventricular spaces. This could be done with fluoroscopic techniques, injecting contrast into the ventricle to ensure the method removes all gasses as expected.

While investigation of a single plane of the brain is presently attainable, the use of a bi-planar HSXR system would allow 3-dimensional displacement to be resolved. This would limit the reliance on embedded contrast markers and could eliminate the assumption of planar motion. Moving forward, this would be a beneficial advancement. Given physical and financial limitations, other marker pattern arrangements, including multiple marker planes, could be utilized to examine outof-plane motion.

The primary result from this study is the relative displacement of different regions of the brain. While this result shows the viability of the methods described, a more valuable result is strain. Additional work in developing the regional displacements into a set of time-resolved strain fields is presently underway. The strain data is the optimal output from future data collections and modifications to the protocol could be introduced to support this. In addition, displacement data and unprocessed tracking data from this study and future studies should also be made available to the broad research community for use in model validation.

\section{Final thoughts}


This document has presented a validated, comprehensive approach to measuring the displacement across the entire brain, with a resolution suitable for focusing on discrete structures. Additionally, it has highlighted some of the complications associated with PMHS studies, including the limitations around repeated impacts to specimens, the sensitivities of embedded contrast design parameters, and the value of performing trials across a broad range of impact characteristics. The implications of the findings reported in this thesis are far reaching. It has long been expected that the heterogeneous nature of the brain would reveal regiondependent impact responses. The observations made from the HSXR video analysis of the PMHS brain motion represent some of the first empirical findings of this phenomena in the deep, small structures of the brain. Of note, large displacements in the corpus callosum are of significance due to the clinically reported damages in even mild cases of TBI. With this study, and the studies that will follow, data suitable for the validation of advanced models and head surrogates will be obtainable. With these advances, further mitigation and understanding of devastating traumatic brain injuries will be possible. 


\section{List of References}

[1] G. of Canada, "Copyright Act, Section 29." https://laws-lois . justice.gc. ca/eng/acts/C-42/index.html, 2020. [Online; accessed 20-September-2020].

[2] U. C. Office, "Copyright Law of the United States (Title 17) , Chapter 1." https://www . copyright.gov/title17/92chap1.html\#107, 2020. [Online; accessed 20-September-2020].

[3] D. P. Rao, S. McFaull, W. Thompson, and G. C. Jayaraman, "Trends in selfreported traumatic brain injury among Canadians, 2005-2014: a repeated cross-sectional analysis," CMAJ Open, vol. 5, pp. E301-E307, apr 2017.

[4] "Surveillance report of traumatic brain injury-related emergency department visits, hospitalizations, and deathsunited states, 2014," Centers for Disease Control and Prevention (2019).

[5] T. El Sayed, A. Mota, F. Fraternali, and M. Ortiz, "Biomechanics of traumatic brain injury," Computer Methods in Applied Mechanics and Engineering, vol. 197, pp. 4692-4701, oct 2008.

[6] F. Mesfin, N. Gupta, A. Hays Shapshak, and et al., "Diffuse Axonal Injury (DAI)." https://www.ncbi.nlm.nih.gov/books/NBK448102/, 2020. [Online; accessed 1-August-2020].

[7] P. C. Blumbergs, G. Scott, J. M. Vis, H. Wainwright, D. A. Simpson, and A. J. Mclean, "Topography of axonal injury as defined by amyloid precursor protein and the sector scoring method in mild and severe closed head injury," Journal of Neurotrauma, vol. 12, no. 4, pp. 565-572, 1995. PMID: 8683607.

[8] D. F. Meaney, B. Morrison, and C. D. Bass, "The Mechanics of Traumatic Brain Injury: A Review of What We Know and What We Need to Know for Reducing Its Societal Burden," 2014. 
[9] C. Prince and M. Bruhns, "Evaluation and treatment of mild traumatic brain injury: The role of neuropsychology," Brain Sciences, vol. 7, p. 105, Aug. 2017.

[10] R. Eppinger, E. Sun, F. Bandak, M. Haffner, N. Khaewpong, M. Maltese, S. Kuppa, T. Nguyen, E. Takhounts, R. Tannous, A. Zhang, and R. Saul, “Development of Improved Injury Criteria for the Assessment of Advanced Automotive Restraint Systems-II," tech. rep., National Highway Traffic Safety Administration, 1999.

[11] "Standard Test Method And Equipment Used In Evaluating The Performance Characteristics Of Headgear/Equipment NOCSAE doc nd 001-17m17b," tech. rep., National Operating Committee on Standards for Athletic Equipment, 2017.

[12] A. Post and T. B. Hoshizaki, "Mechanisms of brain impact injuries and their prediction: A review," Trauma, vol. 14, pp. 327-349, oct 2012.

[13] S. Kleiven, "Finite element modeling of the human head," Department of Aeronautics, Royal Institute of Technology, Licentiate thesis. Report, vol. 9, 012000.

[14] J. S. Raul, C. Deck, R. Willinger, and B. Ludes, "Finite-element models of the human head and their applications in forensic practice," International Journal of Legal Medicine, vol. 122, no. 5, pp. 359-366, 2008.

[15] L. Zhang, K. H. Yang, and A. I. King, "Comparison of Brain Responses Between Frontal and Lateral Impacts by Finite Element Modeling," Journal of Neurotrauma, vol. 18, no. 1, pp. 21-30, 2001.

[16] J. G. Beckwith, R. M. Greenwald, and J. J. Chu, "Measuring head kinematics in football: Correlation between the head impact telemetry system and hybrid III headform," Annals of Biomedical Engineering, vol. 40, pp. 237-248, Oct. 2011.

[17] R. Willinger and D. Baumgartner, "Human head tolerance limits to specific injury mechanisms," International Journal of Crashworthiness, vol. 8, pp. 605617, jan 2003.

[18] Z. Zhou, X. Li, S. Kleiven, C. S. Shah, and W. N. Hardy, "A Reanalysis of Experimental Brain Strain Data: Implication for Finite Element Head Model Validation," SAE Technical Paper Series, vol. 1, no. May 2019, 2019.

[19] R. H. Pudenz and C. H. Shelden, "The lucite calvarium; a method for direct observation of the brain; cranial trauma and brain movement," Journal of neurosurgery, 1946. 
[20] Statistics Canada, “New motor vehicle sales," 2017.

[21] H. De Haven, "Mechanical analysis of survival in falls from heights of fifty to one hundred and fifty feet. 1942.," Injury prevention : journal of the International Society for Child and Adolescent Injury Prevention, 1942.

[22] G. S. Nusholtz, P. Lux, P. Kaiker, and M. A. Janicki, "Head Impact ResponseSkull Deformation and Angular Accelerations," tech. rep., 1984.

[23] A. Al-Bsharat, W. Hardy, K. Yang, T. Khalil, S. Tashman, and A. King, "Brain/skull relative displacement magnitude due to blunt head impact: New experimental data and model," Stapp Car Crash Conference Proceedings, vol. P-350, no. 724, pp. 321-332, 1999.

[24] W. Hardy, C. Foster, M. Mason, K. Yang, A. King, and S. Tashman, “Investigation of head injury mechanisms using neutral density technology and highspeed biplanar x-ray," Stapp car crash journal, vol. 45, pp. 337-68, 122001.

[25] W. Hardy, M. Mason, C. Foster, C. Shah, J. Kopacz, K. Yang, A. King, J. Bishop, M. Bey, W. Anderst, and S. Tashman, "A Study of the Response of the Human Cadaver Head to Impact," Stapp Car Crash Journal, vol. 51, pp. 17-80, 2007.

[26] A. Post, A. Oeur, B. Hoshizaki, and M. D. Gilchrist, "An examination of American football helmets using brain deformation metrics associated with concussion," Materials and Design, vol. 45, 2013.

[27] I. Cernak, "Animal models of head trauma," NeuroRX, vol. 2, pp. 410-422, July 2005.

[28] A. Mazurkiewicz, "Effects of brain gyrification on impact-induced strain measurements with a preliminary study on viable ex vivo porcine brain," 2020.

[29] E. S. Gurdjian, H. R. Lissner, V. R. Hodgson, and L. M. Patrick, “Mechanism of head injury," Neurosurgery, vol. 12, pp. 112-128, 011966.

[30] D. R. Namjoshi, C. Good, W. H. Cheng, W. Panenka, D. Richards, P. A. Cripton, and C. L. Wellington, "Towards clinical management of traumatic brain injury: a review of models and mechanisms from a biomechanical perspective," Disease Models \& Mechanisms, vol. 6, no. 6, pp. 1325-1338, 2013.

[31] V. R. Hodgson, E. S. Gurdjian, and L. M. Thomas, "Experimental skull deformation and brain displacement demonstrated by flash x-ray technique," Journal of Neurosurgery, vol. 25, pp. 549-552, Nov. 1966. 
[32] S. A. Shatsky, "Flash X-Ray Cinematography During Impact Injury," tech. rep., 1973.

[33] R. L. Stalnaker, J. W. Melvin, G. S. Nusholtz, N. M. Alem, and J. B. Benson, "Head impact response," in SAE Technical Paper, SAE International, 021977.

[34] W. Hardy, C. Foster, A. King, and S. Tashman, "Investigation of brain injury kinematics: Introduction of a new technique," ASME, 1997.

[35] A. J. Guettler, "Quantifying the Response of Relative Brain/Skull Motion to Rotational Input in the PMHS Head," Master's thesis, Virginia Polytechnic Institute and State University, 2017.

[36] A. Alshareef, J. S. Giudice, J. Forman, R. S. Salzar, and M. B. Panzer, "A novel method for quantifying human in situ whole brain deformation under rotational loading using sonomicrometry," Journal of Neurotrauma, vol. 35, no. 5, pp. 780-789, 2018.

[37] A. Padgaonkar, K. Krieger, and A. King, "Measurement of angular acceleration of a rigid body using linear accelerometers," Journal of Applied Mechanics, vol. 42, 091975.

[38] P. Martin, G. Hall, J. Crandall, and W. Pilkey, "Measuring the acceleration of a rigid body," Shock and Vibration, vol. 5, pp. 211-224, 011998.

[39] J. J. Crisco, J. J. Chu, and R. M. Greenwald, "An Algorithm for Estimating Acceleration Magnitude and Impact Location Using Multiple Nonorthogonal Single-Axis Accelerometers," Journal of Biomechanical Engineering, vol. 126, pp. 849-854, dec 2004.

[40] K. L. O'Connor, S. Rowson, S. M. Duma, and S. P. Broglio, "Head-ImpactMeasurement Devices: A Systematic Review.," Journal of athletic training, vol. 52, pp. 206-227, mar 2017.

[41] A. M. Nahum, R. Smith, and C. C. Ward, "Intracranial pressure dynamics during head impact," in SAE Technical Paper Series, SAE International, Feb. 1977.

[42] A. I. King, K. H. Yang, and W. N. Hardy, "Recent firsts in cadaveric impact biomechanics research," Clinical Anatomy, 2011. 
[43] Y. Feng, T. M. Abney, R. J. Okamoto, R. B. Pless, G. M. Genin, and P. V. Bayly, "Relative brain displacement and deformation during constrained mild frontal head impact," Journal of The Royal Society Interface, vol. 7, pp. 1677-1688, May 2010.

[44] G. Selvik, "Roentgen stereophotogrammetry," Acta Orthopaedica Scandinavica, vol. 60, no. sup232, pp. 1-51, 1989.

[45] R. Tranberg, T. Saari, R. Zgner, and J. Krrholm, "Simultaneous measurements of knee motion using an optical tracking system and radiostereometric analysis (rsa)," Acta Orthopaedica, vol. 82, no. 2, pp. 171-176, 2011.

[46] I. Sbalzarini and P. Koumoutsakos, "Feature point tracking and trajectory analysis for video imaging in cell biology," Journal of Structural Biology, vol. 151, pp. 182-195, Aug. 2005.

[47] S. Magnan, "Analysis of Radiographic Contrast Markers for X-ray Digital Image Correlation of Tissue-Simulants under Dynamic Load," Master's thesis, Carleton University, 2018.

[48] The Mathworks, Inc., Natick, Massachusetts, MATLAB version 9.5.0.944444 (R2018b), 2018.

[49] S. Leutenegger, M. Chli, and R. Siegwart, "Brisk: Binary robust invariant scalable keypoints," pp. 2548-2555, 112011.

[50] N. Yoganandan, F. A. Pintar, J. Zhang, and J. L. Baisden, "Physical properties of the human head: Mass, center of gravity and moment of inertia," 2009.

[51] G. C. Roush, "Finding Cadaveric Human Head Masses and Center of Gravity : A Comparison of Direct Measurement to 3D Modeling," 2010.

[52] S. Seltzer, "Tables of x-ray mass attenuation coefficients and mass energyabsorption coefficients, nist standard reference database 126," 1995.

[53] T. Whyte, J. Liu, V. Chung, S. McErlane, Z. Abebe, K. McInnes, C. Wellington, and P. Cripton, "Technique and preliminary findings for in vivo quantification of brain motion during injurious head impacts," Journal of Biomechanics, vol. 95, p. 109279, Oct. 2019.

[54] K. Shi, D. Zhang, J. Ren, C. Yao, and Y. Yuan, "Multiobjective optimization of surface integrity in milling TB6 alloy based on Taguchi-grey relational analysis," Advances in Mechanical Engineering, vol. 2014, no. March, 2014. 
[55] R. N. Palchesko, L. Zhang, Y. Sun, and A. W. Feinberg, "Development of Polydimethylsiloxane Substrates with Tunable Elastic Modulus to Study Cell Mechanobiology in Muscle and Nerve," PLoS ONE, vol. 7, no. 12, 2012.

[56] A. Lebar, A Study of Particle Surface Effects on the Spall Strength of ParticleReinforced Polymer Matrix Composites. PhD thesis.

[57] H. Atil and U. Yakut, "A different approach of experimental design: Taguchi method," Pakistan Journal of Biological Sciences, vol. 3, 092000.

[58] T. Taner and J. Antony, "Applying Taguchi methods to health care.," International journal of health care quality assurance incorporating Leadership in health services, vol. 19, no. 1, 2006.

[59] B. M. Gopalsamy, B. Mondal, and S. Ghosh, "Taguchi method and anova: An approach for process parameters optimization of hard machining while machining hardened steel," Journal of Scientific and Industrial Research, vol. 68, no. 8, pp. 686-695, 2009.

[60] S. V. R. Athreya and D. Venkatesh, "Application of taguchi method for optimization of process parameters in improving the surface roughness of lathe facing operation," International Refereed Journal of Engineering and Science, vol. 1, pp. 13-19, 2012.

[61] A. Rosset, L. Spadola, and O. Ratib, "OsiriX: An open-source software for navigating in multidimensional DICOM images," Journal of Digital Imaging, vol. 17, pp. 205-216, June 2004.

[62] E. S. Walsh, M. Kendall, · A. Post, A. Meehan, and ·. T. B. Hoshizaki, "Comparative analysis of Hybrid III neckform and an unbiased neckform," vol. 21, pp. 479-485, 2018.

[63] S. Munakomi and J. Das, "Ventriculostomy," 2020. 


\section{Appendix A}

\section{Artificial Cerebrospinal Fluid Formula}

The Artificial Cerebrospinal Fluid (aCSF) is composed of two solutions. The stock solution can be prepared in advance of experimentation and frozen for long term storage. The stock solution is most easily made in $500 \mathrm{~mL}$ batches, due to the relatively small masses of added components. The sugar solution must be prepared on the day of the experiment. In preparing the sugar solution, care must be taken to ensure the complete dissolution of each component before adding the next. The final aCSF is 1-part stock solution and 9-parts sugar solution, by volume. For higher yields, the components can be scaled linearly.

\begin{tabular}{|c|c|}
\hline Stock Solution & Sugar Solution \\
\hline $500 \mathrm{~mL} \mathrm{DH}_{2} \mathrm{O}$ (Distilled Water) & $450 \mathrm{~mL} \mathrm{DH}_{2} \mathrm{O}$ (Distilled Water) \\
\hline $36.233 \mathrm{~g} \mathrm{NaCl}$ (Sodium Chloride) & $0.901 \mathrm{~g} \mathrm{C}_{6} \mathrm{H}_{1} 2 \mathrm{O}_{6}$ (Glucose) \\
\hline $1.119 \mathrm{~g} \mathrm{KCl}$ (Potassium Chloride) & $1.092 \mathrm{~g} \mathrm{NaHCO}_{3}$ (Sodium Bicarbonate) \\
\hline $\begin{array}{l}1.602 \mathrm{~g} \mathrm{MgSO}_{4} \text { (Magnesium Sulfate) } \\
0.84 \mathrm{~g} \mathrm{NaH}_{2} \mathrm{PO}_{4} \text { (Sodium Phosphate) }\end{array}$ & $0.184 \mathrm{~g} \mathrm{CaCl}_{2}$ (Calcium Chloride) \\
\hline
\end{tabular}




\section{Appendix B}

\section{Apparatus Drawings}

Dimensioned reference drawings for apparatus referenced in Chapter 5 follow. 


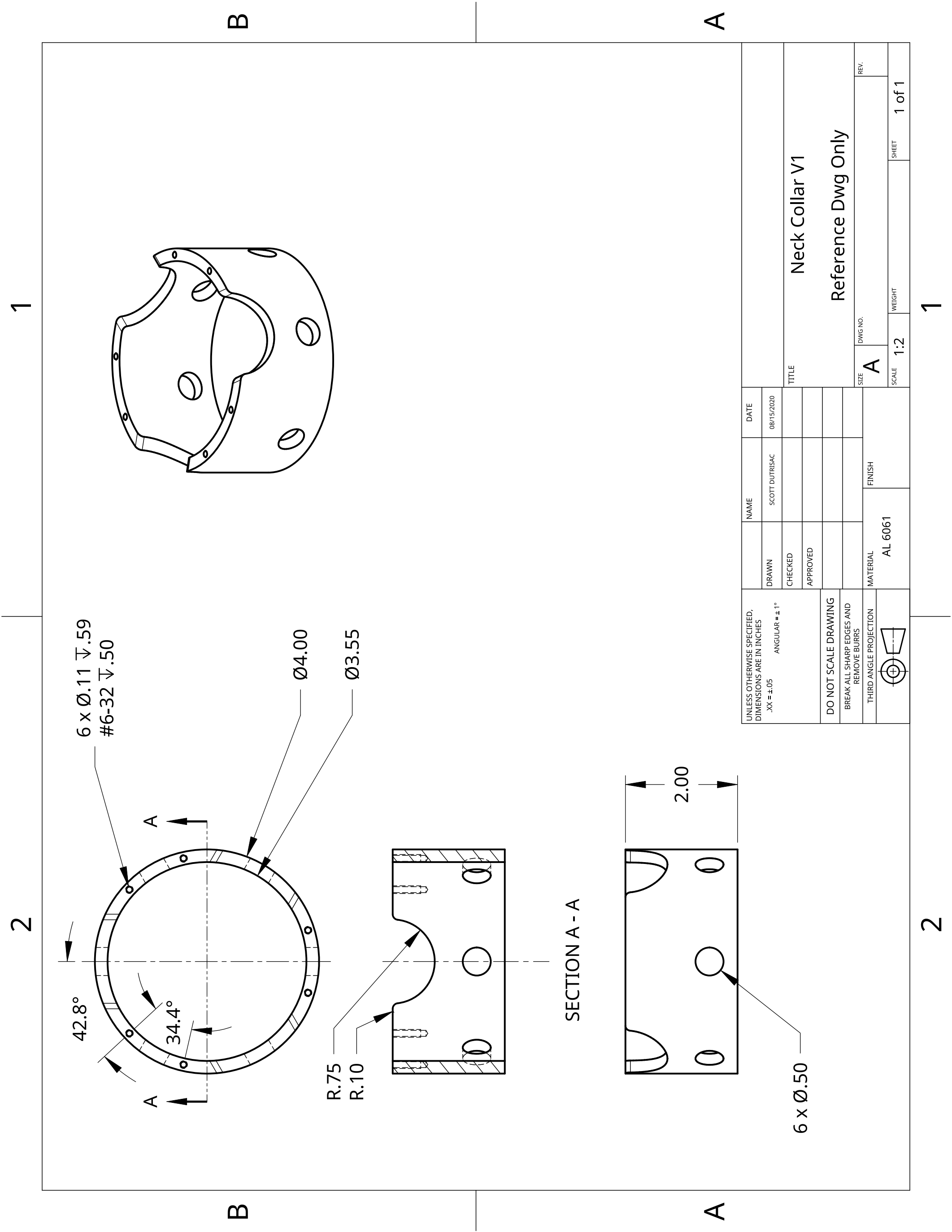



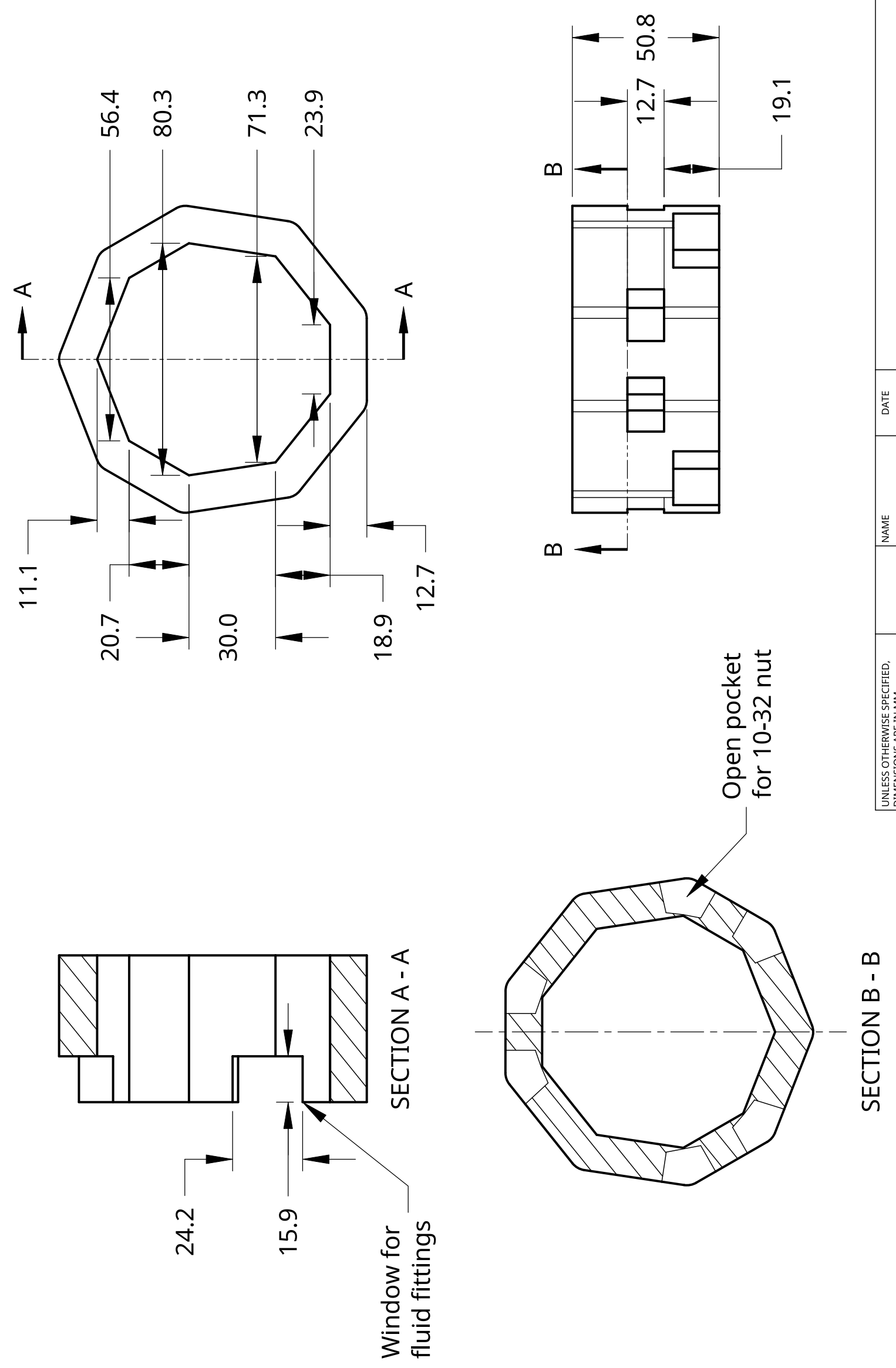

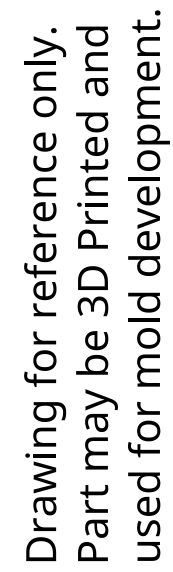

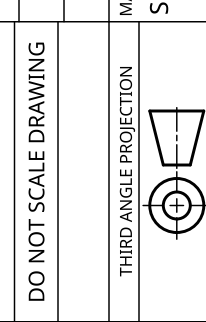

N 
$\varangle$
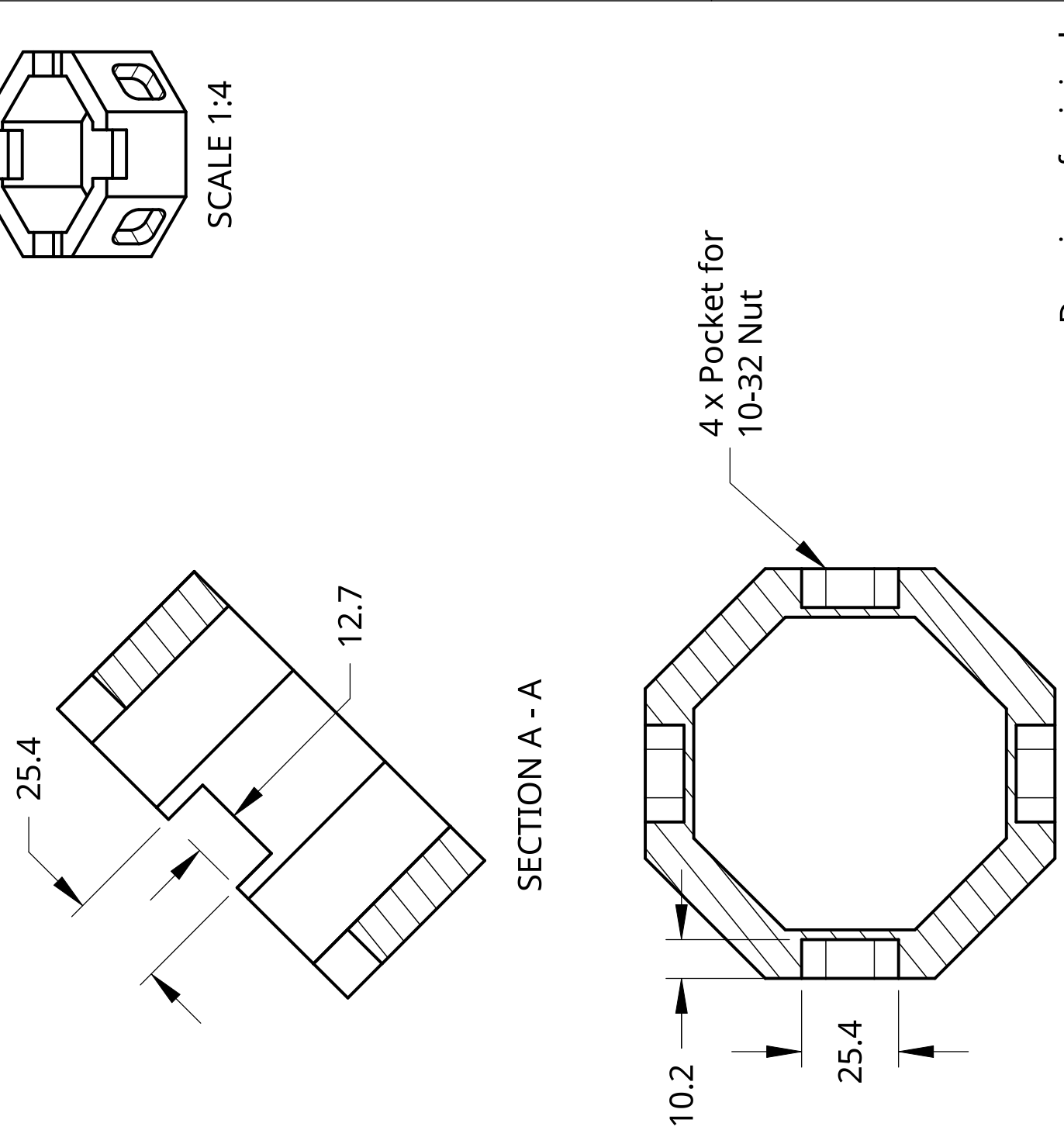

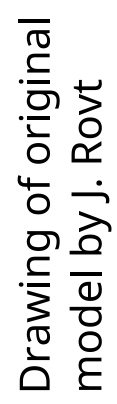

$-$
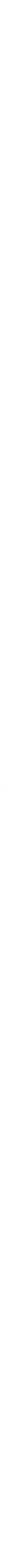

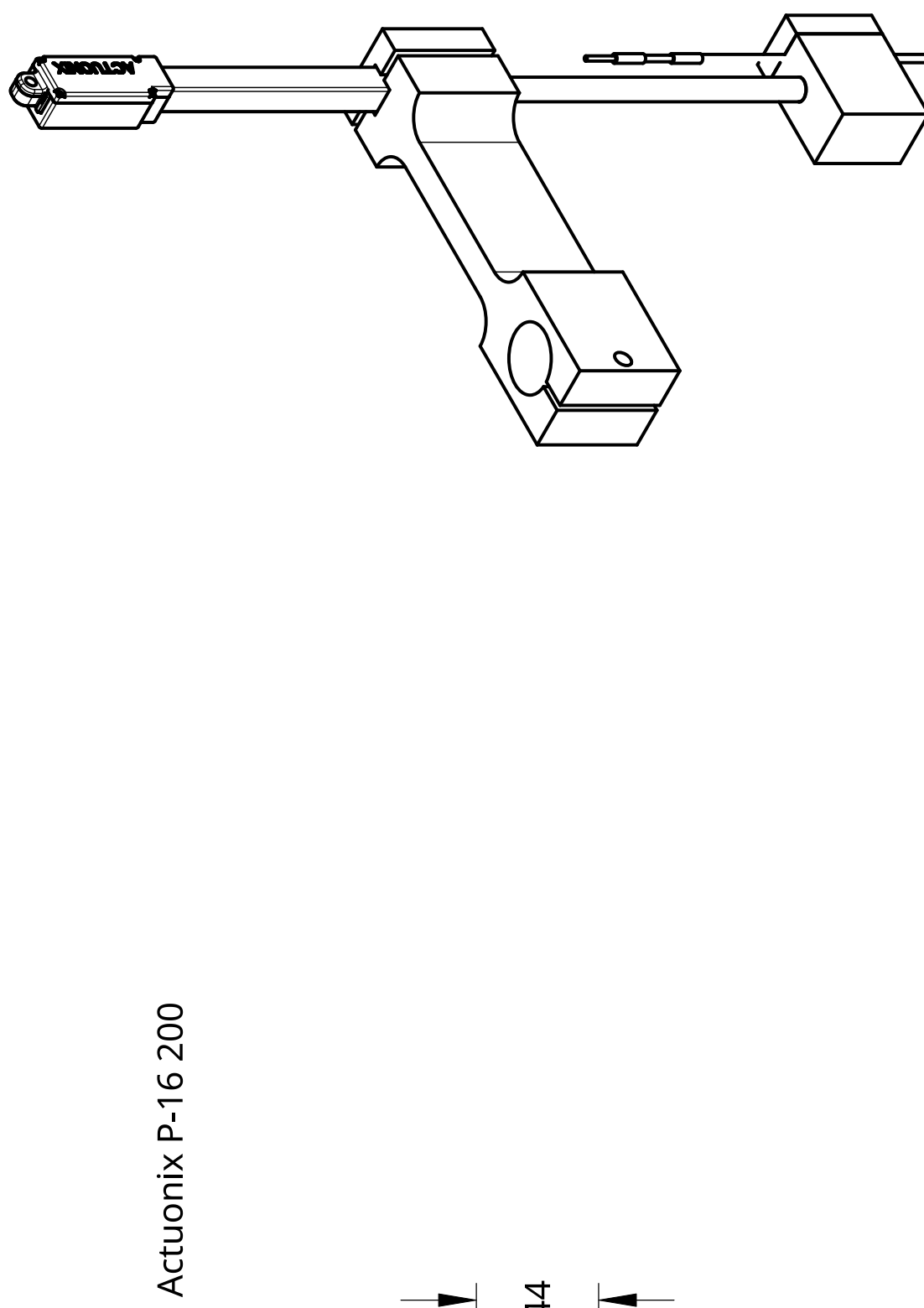

$N$

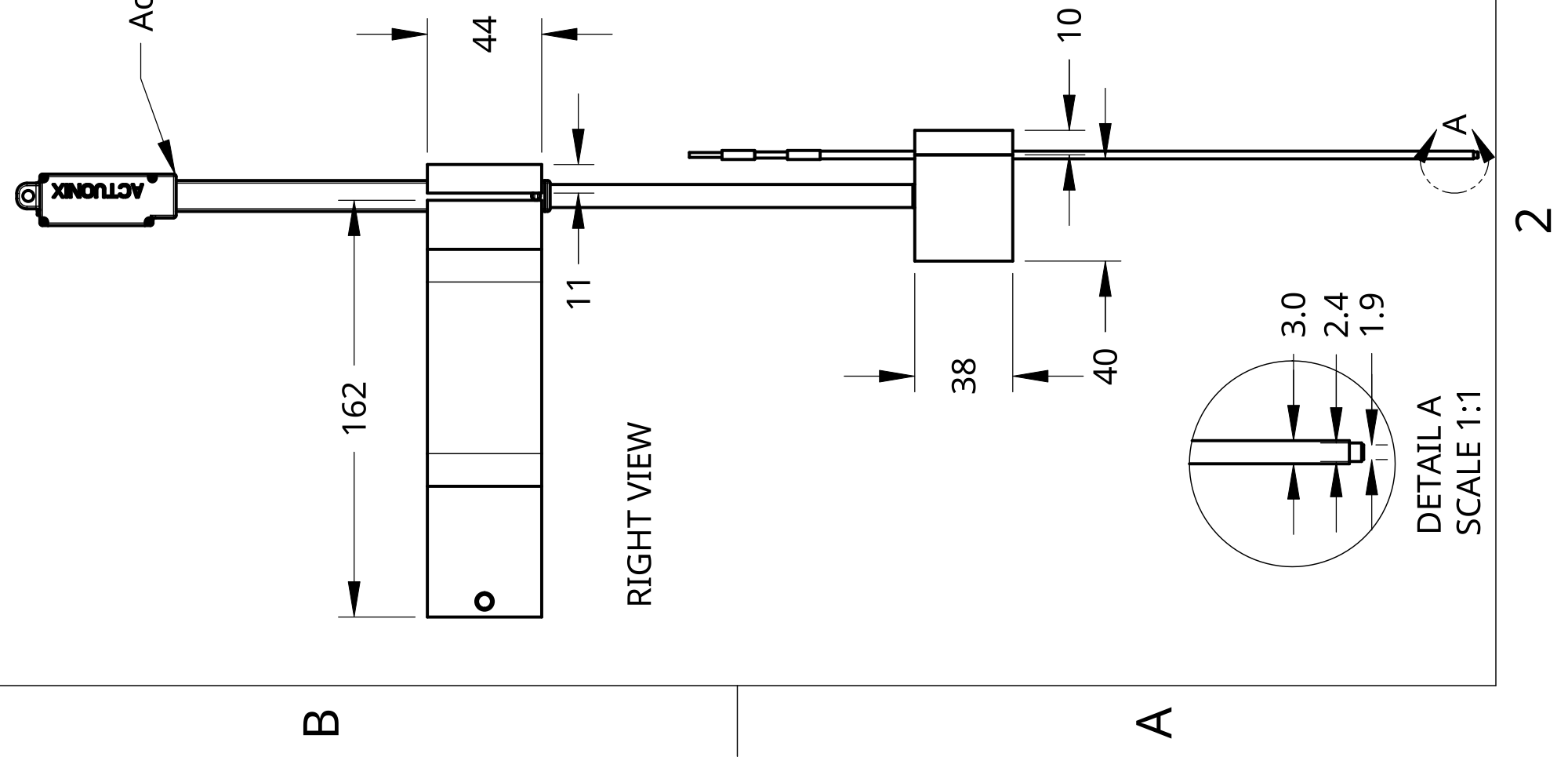



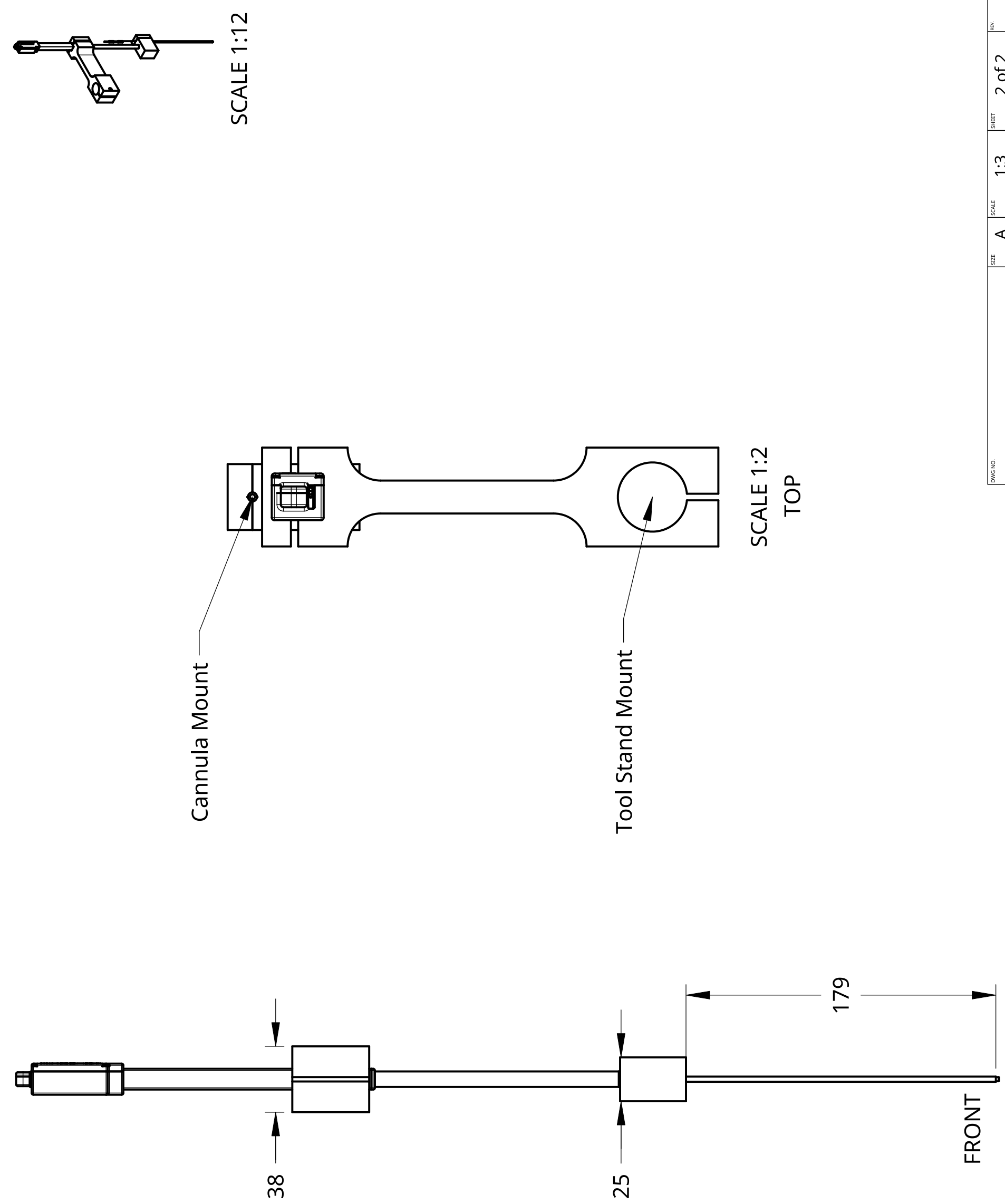


\section{Appendix C}

\section{Impact Protocol}

The study outlined in this thesis covers the second and third specimens associated with the impact protocol listed below. The first specimen, not discussed in this thesis, was used for methodology evaluation, but did not yield sufficient data for discussion. The tests for the first specimen will be performed using one of the two "TBD" specimens. For each specimen, five impacts are listed, however if specimens appear to be in tact for further testing, repeated impacts are attempted. This protocol was developed by the research group and may change due to PMHS availability, or as experimental focuses shift. 
Table 13: Planned impact protocol for PMHS specimens

\begin{tabular}{ccc|cccccc} 
& & & \multicolumn{6}{|c}{ Impact Speed $(\mathrm{m} / \mathrm{s})$} \\
Specimen & Site/Plane & Impactor Compliance & 1 & 2 & 3 & 4 & 5 & 6 \\
\hline 1 & Front/Sagittal & High & 1.5 & 1.5 & 3 & 3 & 4.5 & 4.5 \\
2 & Front/Sagittal & High & 0.5 & 1 & 1.5 & 3 & 4.5 & 6 \\
3 & Front/Sagittal & Low & 0.5 & 1 & 1.5 & 2 & $2.5+$ & - \\
4 & Front/Sagittal & Medium & 0.5 & 1 & 1.5 & 3 & 4.5 & - \\
\hline 5 & Rear/Sagittal & High & 0.5 & 1 & 1.5 & 3 & 4.5 & 6 \\
6 & Rear/Sagittal & Medium & 0.5 & 1 & 1.5 & 3 & 4.5 & - \\
7 & Rear/Sagittal & Low & 0.5 & 1 & 1.5 & 2 & $2.5+$ & - \\
\hline 8 & Side/Coronal & High & 0.5 & 1 & 1.5 & 3 & 4.5 & 6 \\
9 & Side/Coronal & & 0.5 & 1 & 1.5 & 3 & 4.5 & - \\
10 & Side/Coronal & Low & 0.5 & 1 & 1.5 & 2 & $2.5+$ & - \\
11 & Side/Coronal & High & 0.5 & 1 & 1.5 & 3 & 4.5 & 6 \\
\hline $12^{*}$ & Front/Sagittal & Medium & 0.5 & 1 & 1.5 & 3 & 4.5 & - \\
13 & Rear/Sagittal & Low & 0.5 & 1 & 1.5 & 2 & $2.5+$ & - \\
\hline 14 & TBD & & & & & & & \\
15 & TBD & & & & & & &
\end{tabular}




\section{Appendix D}

\section{Additional Tables and Plots}

This appendix contains additional reference data not discussed in Chapter 6. The measurements listed in Table 14 correspond to the anthropometric measurements found in Figure 30 in Chapter 5

Table 14: Specimen physical and anthropometric data

\begin{tabular}{c|cc} 
& 2842 & 2871 \\
\hline Age & 66 & 57 \\
Sex & F & F \\
Post-Mortem Time (h) & 54 & 36 \\
Mass (kg) & 2.87 & 3.32 \\
\hline Measurements (cm) & & \\
\hline a & 12.5 & 15.0 \\
b & 12.0 & 14.0 \\
c & 16.0 & 17.0 \\
d & 5.0 & 8.0 \\
e & 16.0 & 21.0 \\
f & 30.5 & 33.0 \\
g & 17.0 & 18.0 \\
h & 52.0 & 57.0
\end{tabular}

Table 15 lists the offset distances from the accelerometer package centre to the CG of the head. Figure 66 shows the coordinate system used, with the blue dot 
representing the accelerometer centre.

Table 15: Offset distances for accelerometer to CG

\begin{tabular}{c|cc} 
& \multicolumn{2}{|c}{ Offset $(\mathrm{mm})$} \\
Direction & 2842 & 2871 \\
$\mathrm{x}$ & 2 & 9 \\
$\mathrm{y}$ & -84 & -84 \\
$\mathrm{z}$ & 153 & 171
\end{tabular}

Figure 66: Coordinate System for Accelerometer Offset

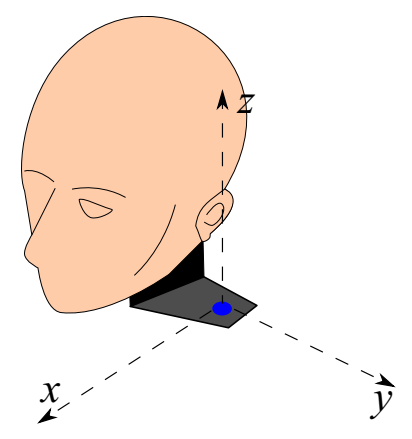


Figure 67 shows a comparison between C288-T1 performed by Hardy (2007) [25] and 2842-4 and 2871-7.
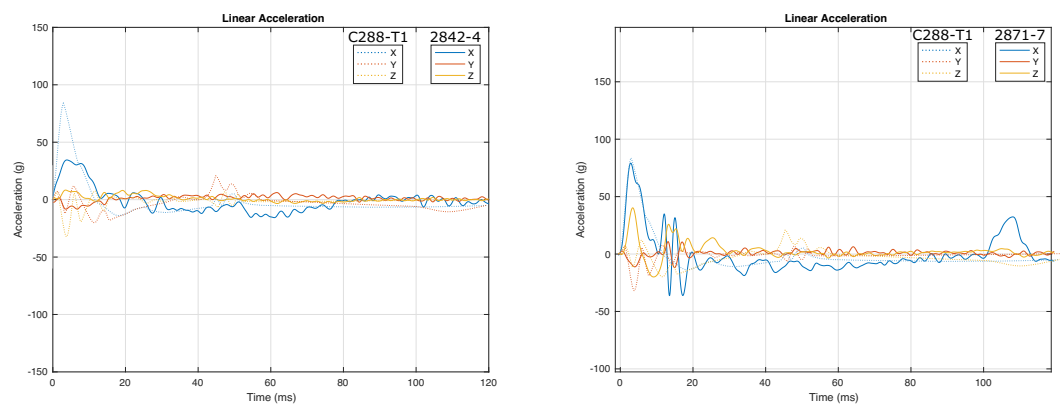

Figure 67: Left: C288-T1 compared to 2842-4. The peak linear acceleration is lower in 2842-4, with a longer duration, as expected due to the high-compliance impactor. Right: C288-T1 compared to 2871-7. The combined speed $(3.7 \mathrm{~m} / \mathrm{s})$ and impactor cap combination in 2871-7 replicates the acceleration profile of the $3.3 \mathrm{~m} / \mathrm{s}$ helmeted impact in C288-T1. Both figures are adapted from Hardy (2007) [25]

The figures below provide the raw pressure signal and compensated pressure measurements for Specimen 2871, Impacts 1-5 and 7. 


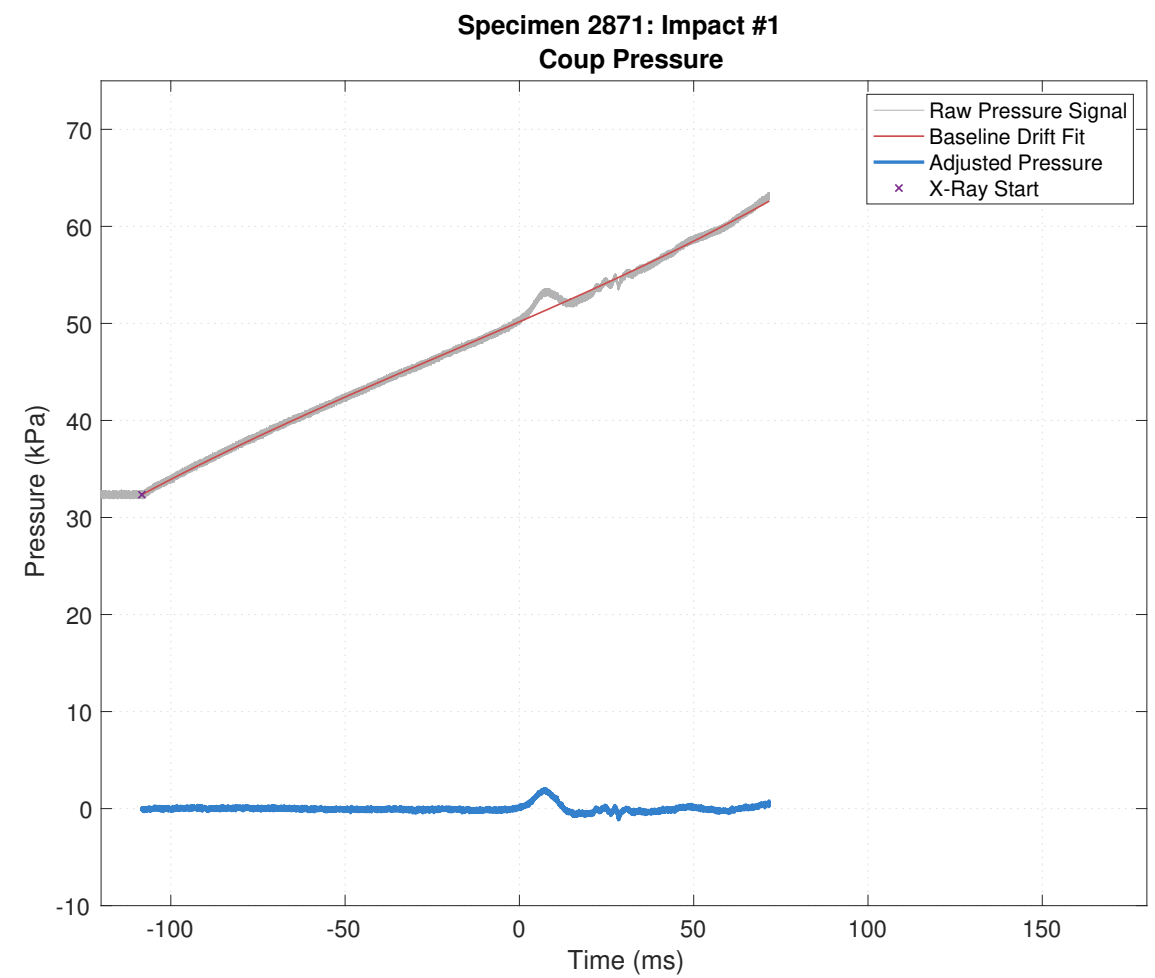

Figure 68: Coup pressure for Specimen 2871, Impact 1, $1.0 \mathrm{~m} / \mathrm{s}$.

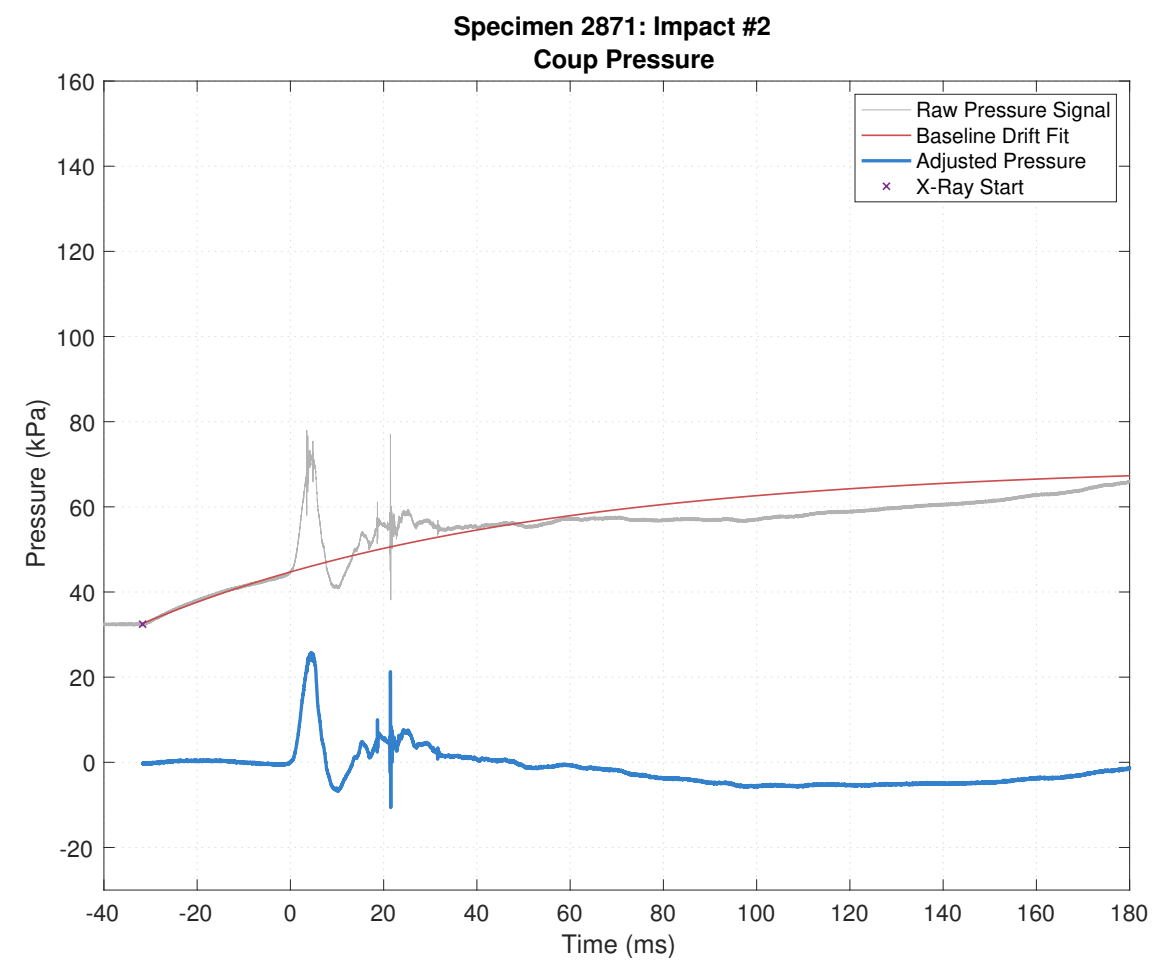

Figure 69: Coup pressure for Specimen 2871, Impact 2, $2.1 \mathrm{~m} / \mathrm{s}$. 


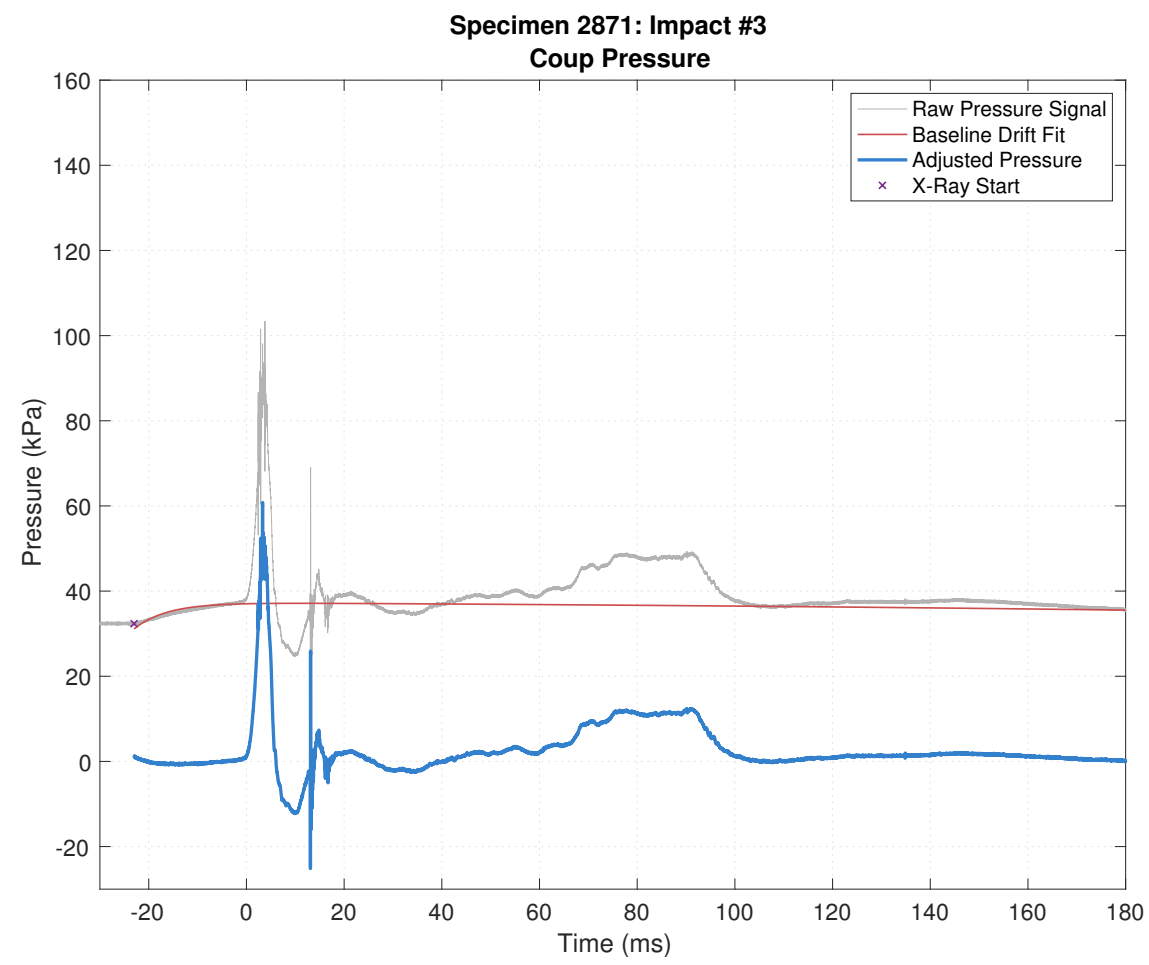

Figure 70: Coup pressure for Specimen 2871, Impact 3, $3.0 \mathrm{~m} / \mathrm{s}$.

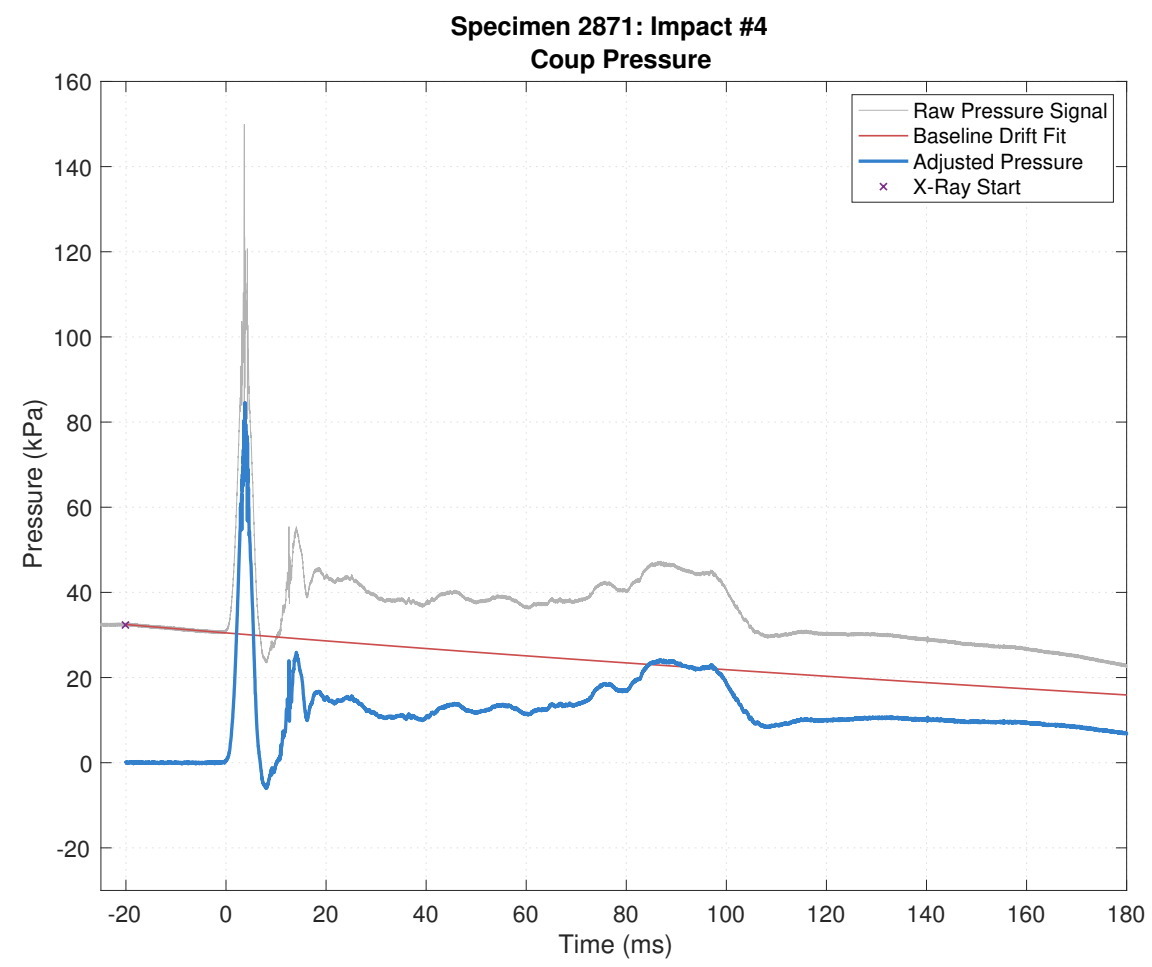

Figure 71: Coup pressure for Specimen 2871, Impact 4, 3. m/s. 


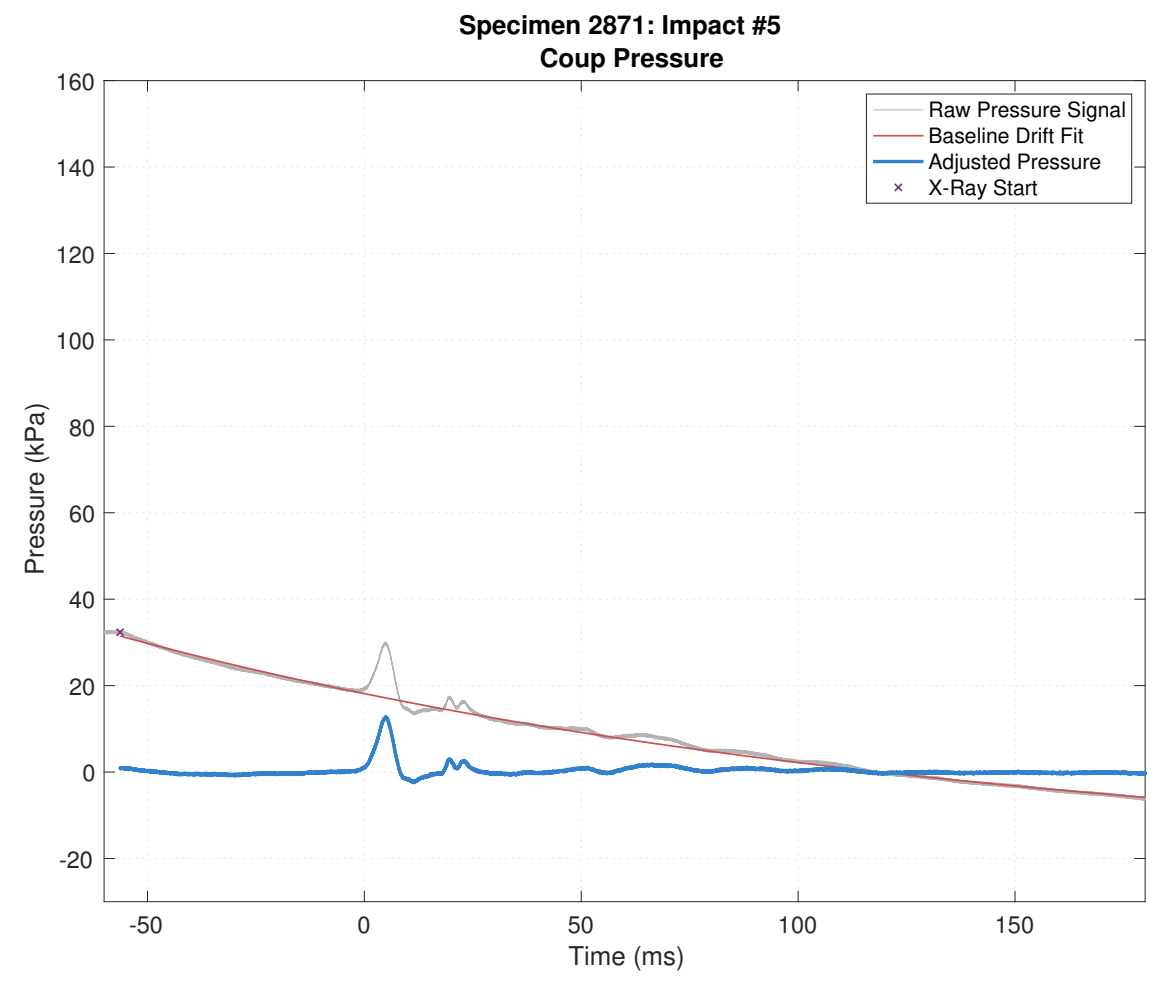

Figure 72: Coup pressure for Specimen 2871, Impact 5, $1.2 \mathrm{~m} / \mathrm{s}$.

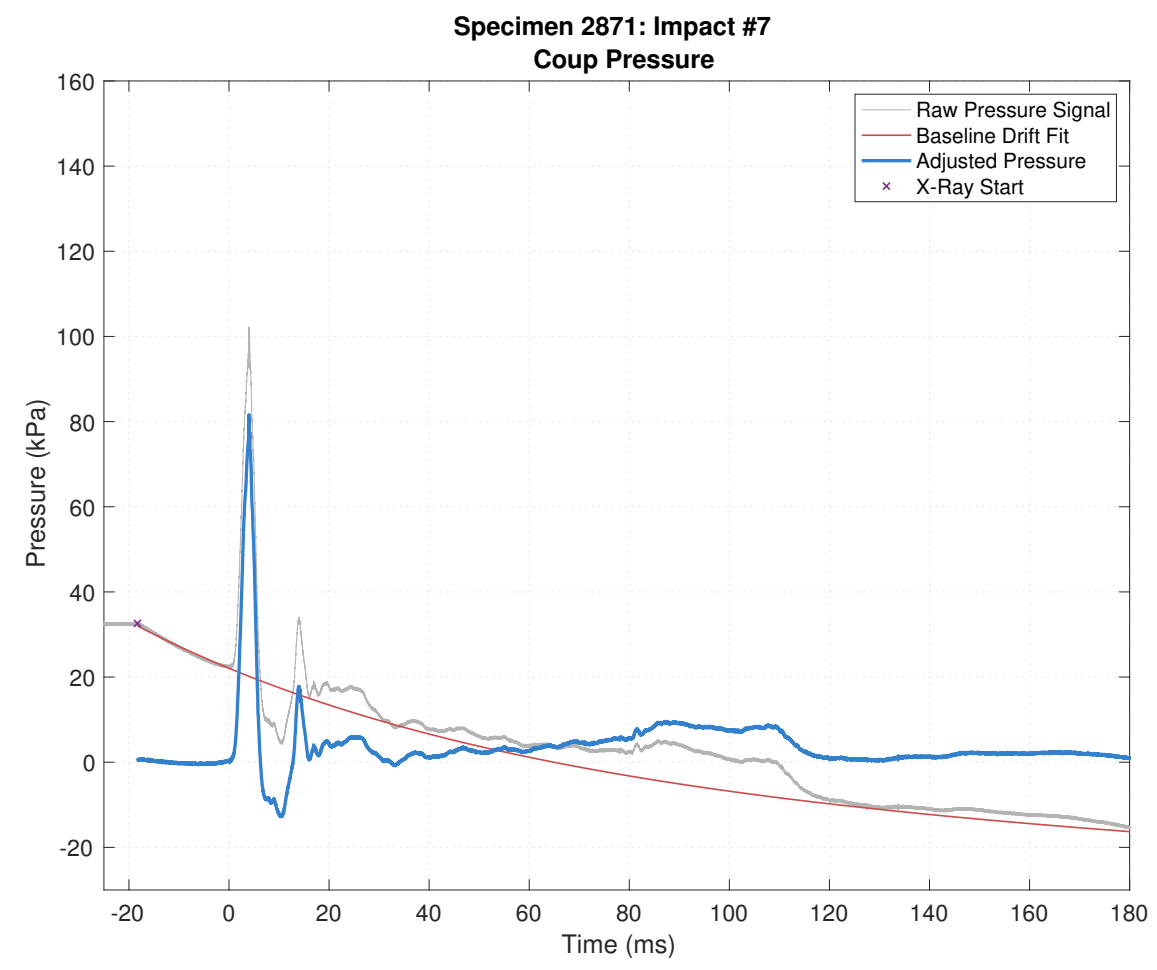

Figure 73: Coup pressure for Specimen 2871, Impact 7, $3.7 \mathrm{~m} / \mathrm{s}$. 
The millimetre-pixel conversion factors used for each image set are shown in Table 16. Subsequent figures provide further information on displacements observed for impacts of Specimens 2842 and 2871.

Table 16: Millimetre-pixel scales

\begin{tabular}{ccc} 
Specimen & Impact & Scale $(\mathrm{mm} / \mathrm{px})$ \\
\hline 2842 & 2 & 0.33 \\
& 3 & 0.33 \\
& 4 & 0.33 \\
& 7 & 0.33 \\
2871 & 10.32 & \\
& 2 & 0.31 \\
& 3 & 0.30 \\
& 4 & 0.31 \\
& 5 & 0.32 \\
& 6 & 0.31 \\
& 7 & 0.31 \\
\hline
\end{tabular}




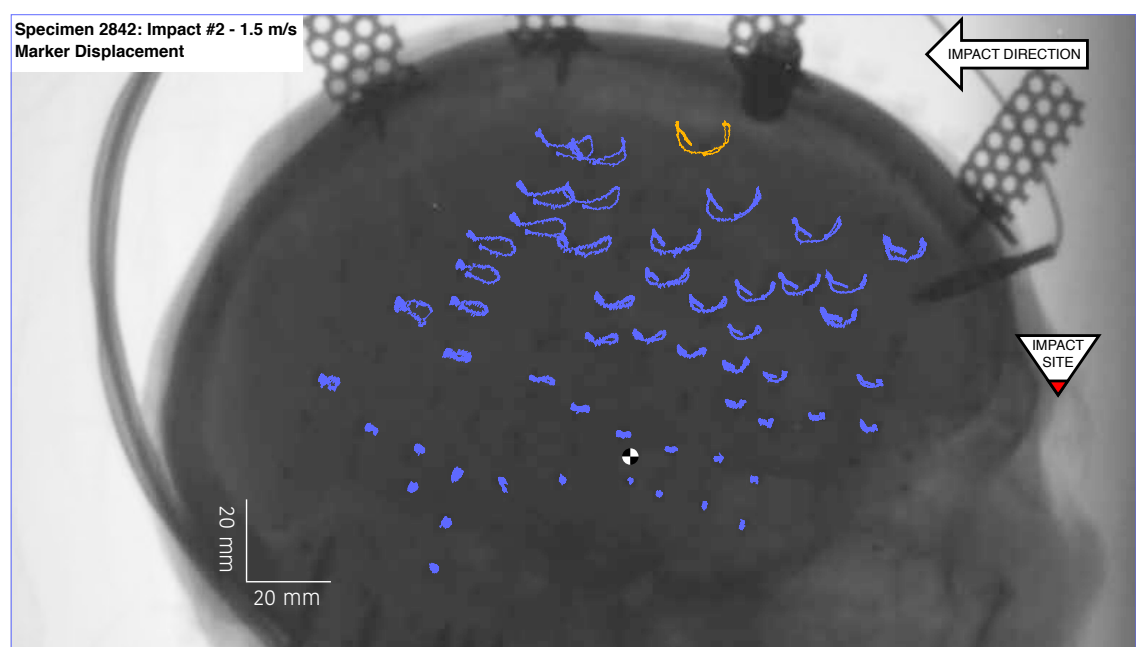

Figure 74: Displacement paths for Specimen 2842, Impact 2, $1.5 \mathrm{~m} / \mathrm{s}$. Paths represent full marker motion until approximately $180 \mathrm{~ms}$ after impact. Peak displacement trace in orange.

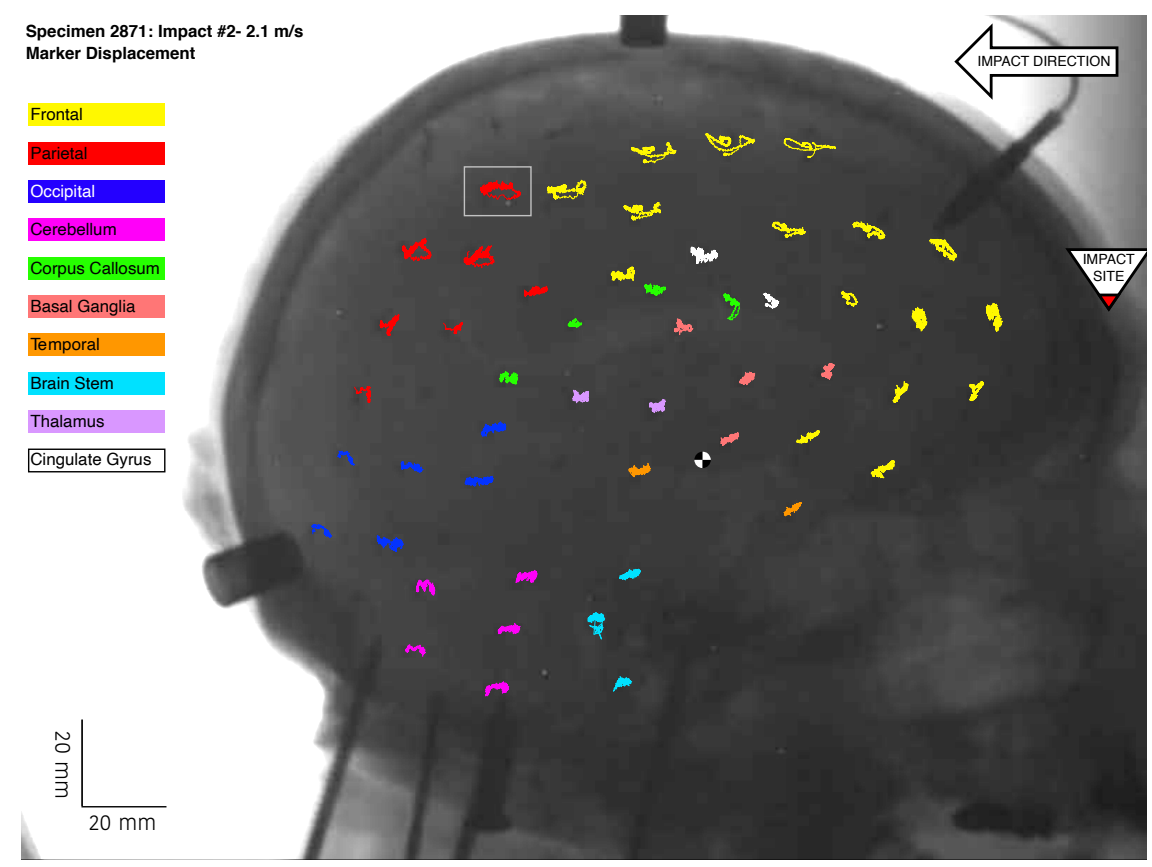

Figure 75: Displacement paths for 2871-2, $2.1 \mathrm{~m} / \mathrm{s}, 0 \mathrm{~ms}<t_{\text {impact }}<180 \mathrm{~ms}$. Regional colour coding per post-experiment MRI. A rotational pattern can be seen across the entire brain, with larger displacements further from the centre of rotation, which is offset from the CG. Looping patterns are present throughout. 


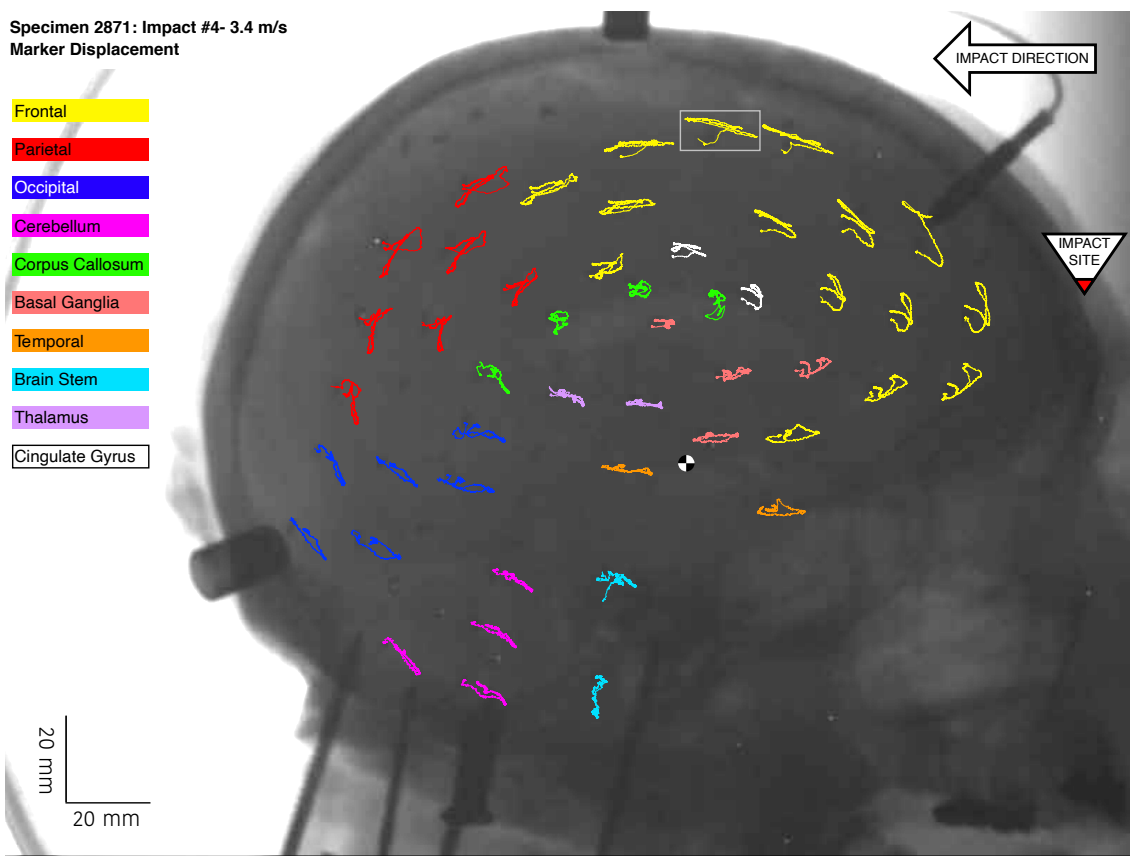

Figure 76: Displacement paths for Specimen 2871, Impact 4, $3.4 \mathrm{~m} / \mathrm{s}$. Paths represent full marker motion until approximately $180 \mathrm{~ms}$ after impact. Regions as determined by neurosurgeon partners. Peak displacement trace highlighted with grey box. 
The following figures (Figure 77 to Figure 146) show the regional displacements for each of the identified regions in Chapter 6

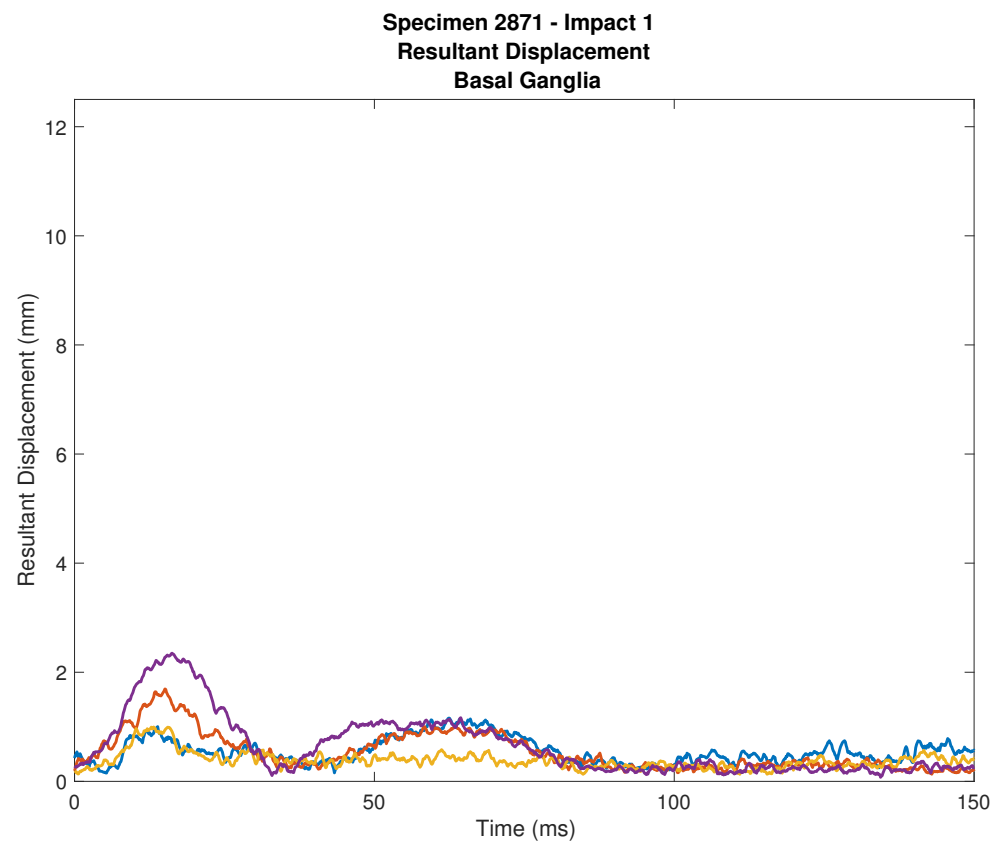

Figure 77: Displacement of basal ganglia - 2871-1

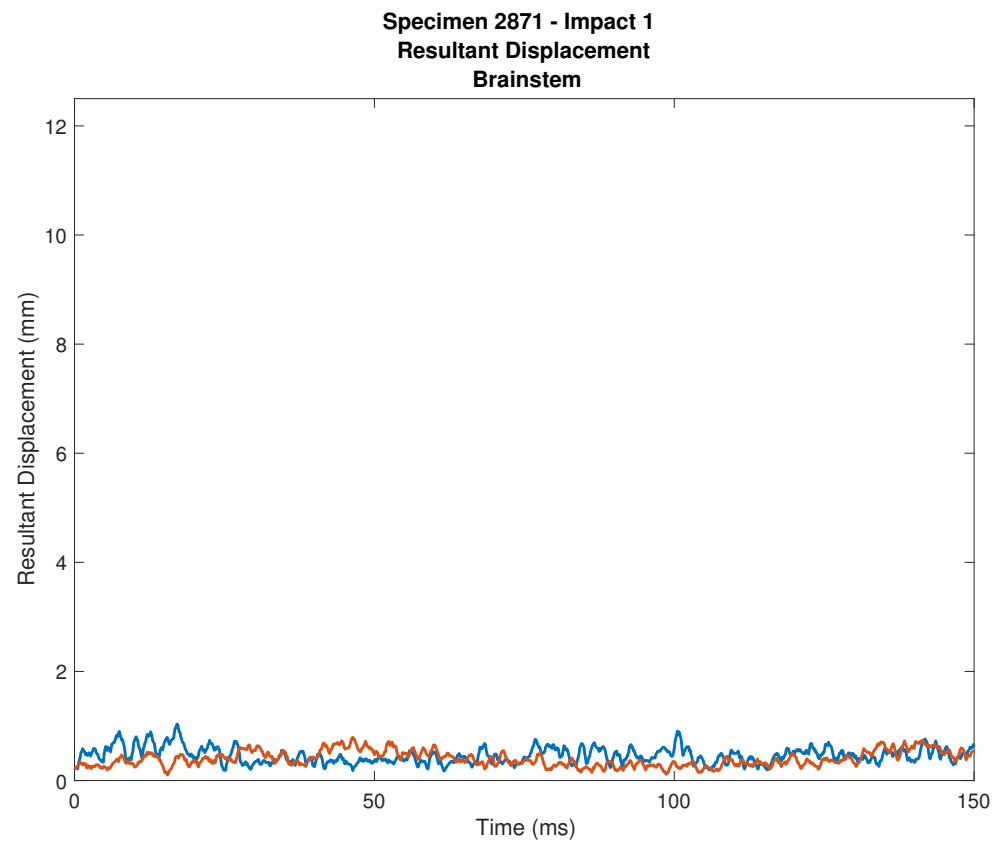

Figure 78: Displacement of brainstem - 2871-1 


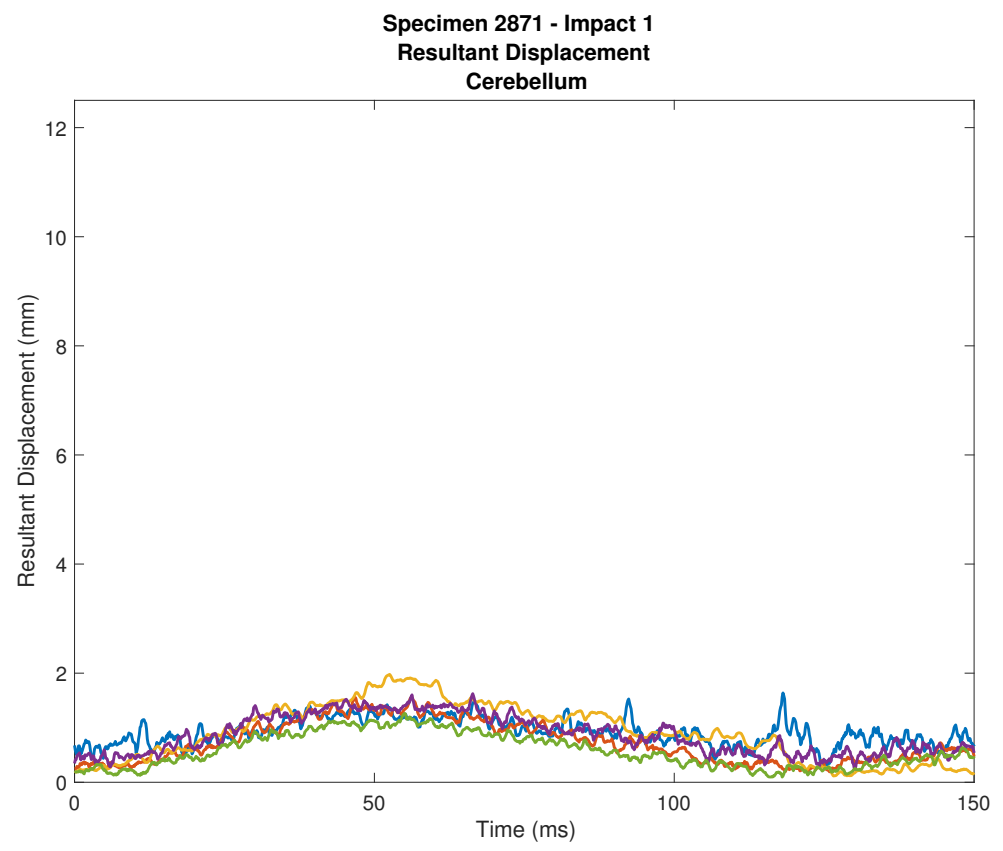

Figure 79: Displacement of cerebellum - 2871-1

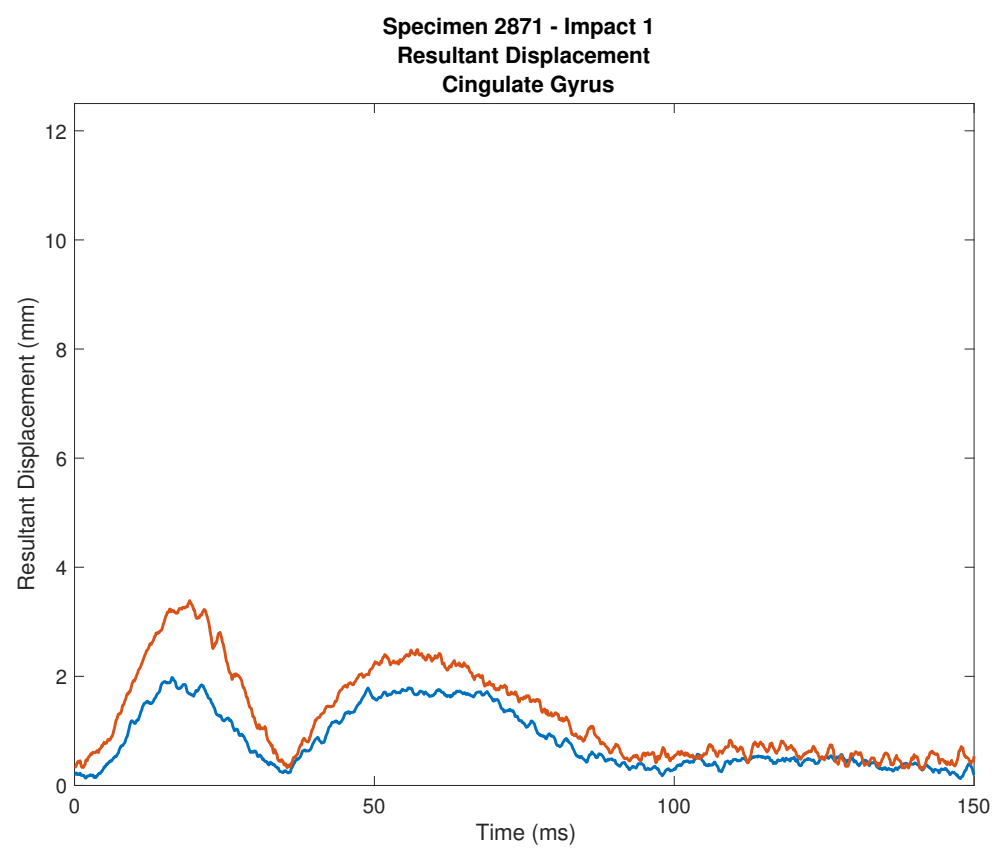

Figure 80: Displacement of cingulate gyrus - 2871-1 


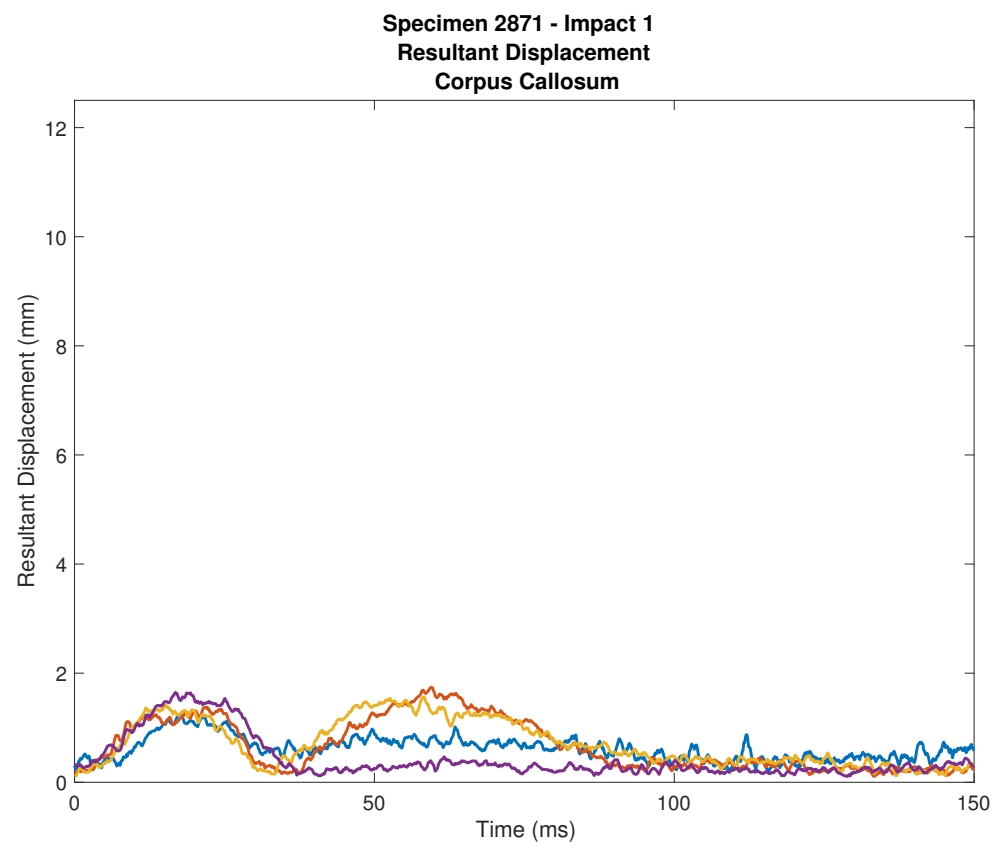

Figure 81: Displacement of corpus callosum - 2871-1

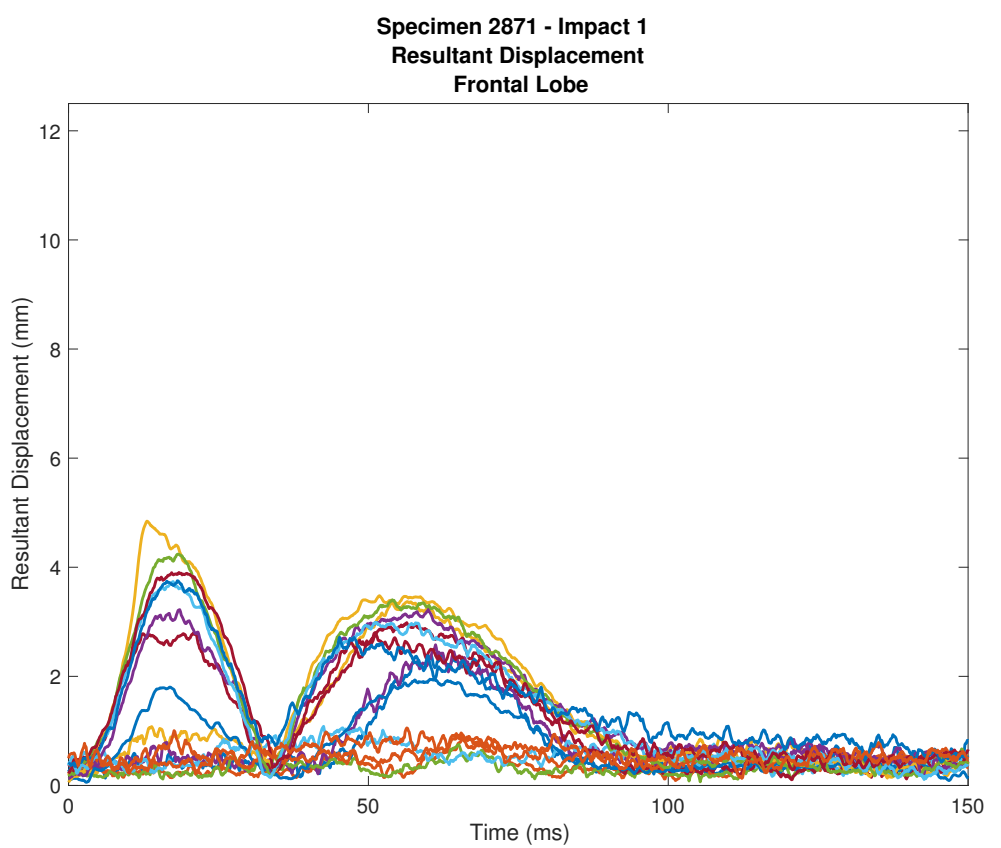

Figure 82: Displacement of frontal lobe - 2871-1 


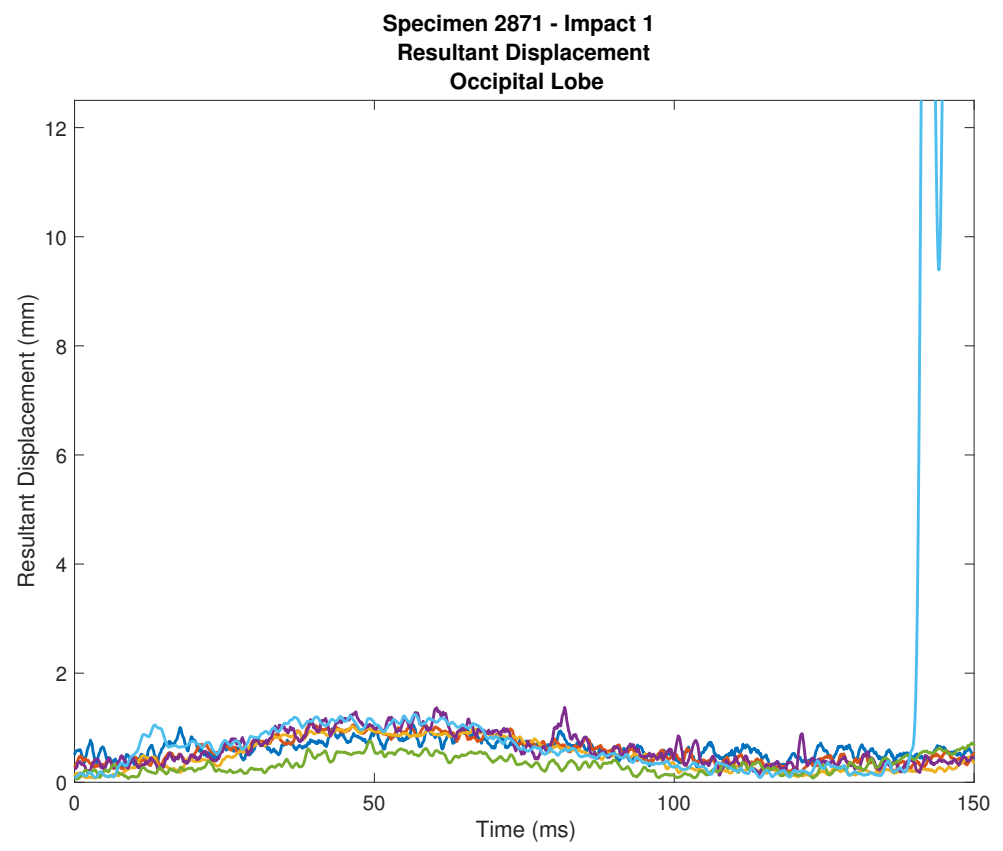

Figure 83: Displacement of occipital lobe - 2871-1

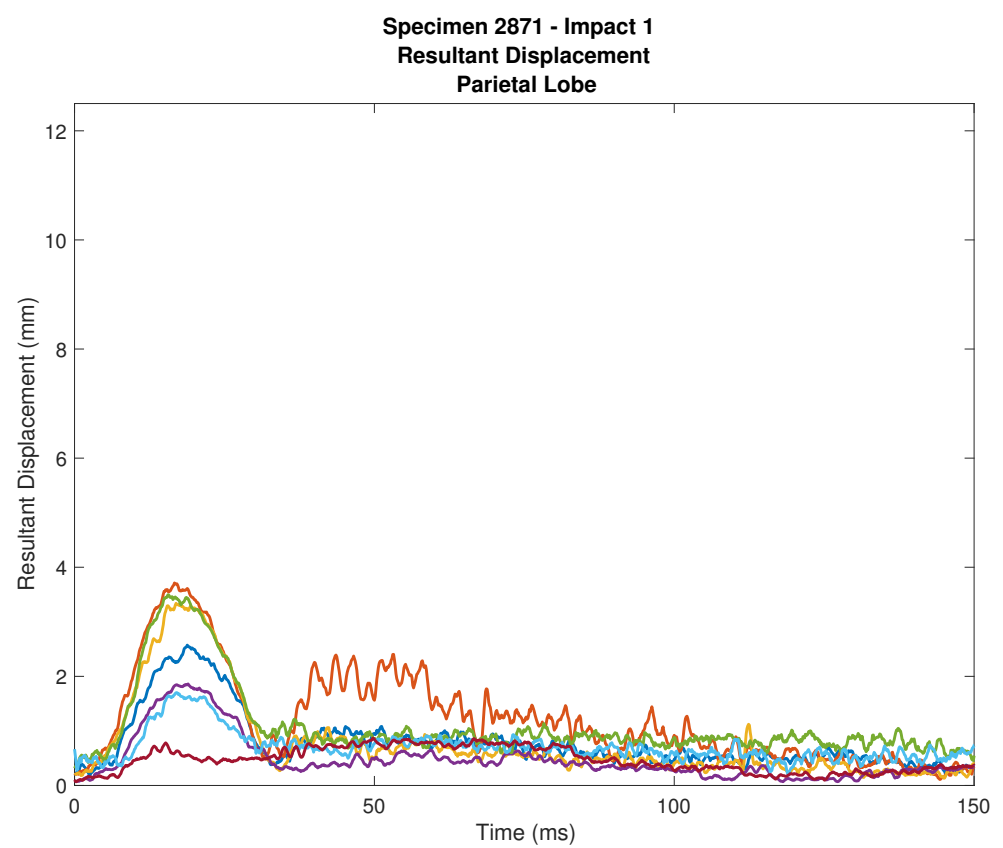

Figure 84: Displacement of parietal lobe - 2871-1 


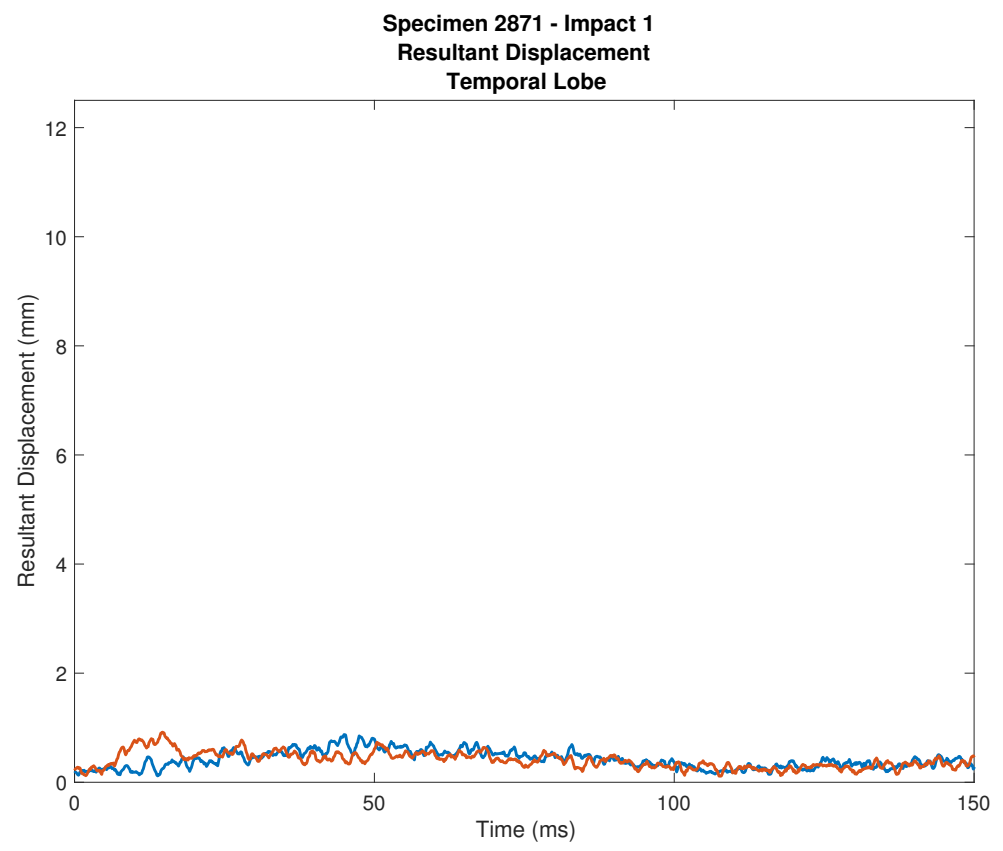

Figure 85: Displacement of temporal lobe - 2871-1

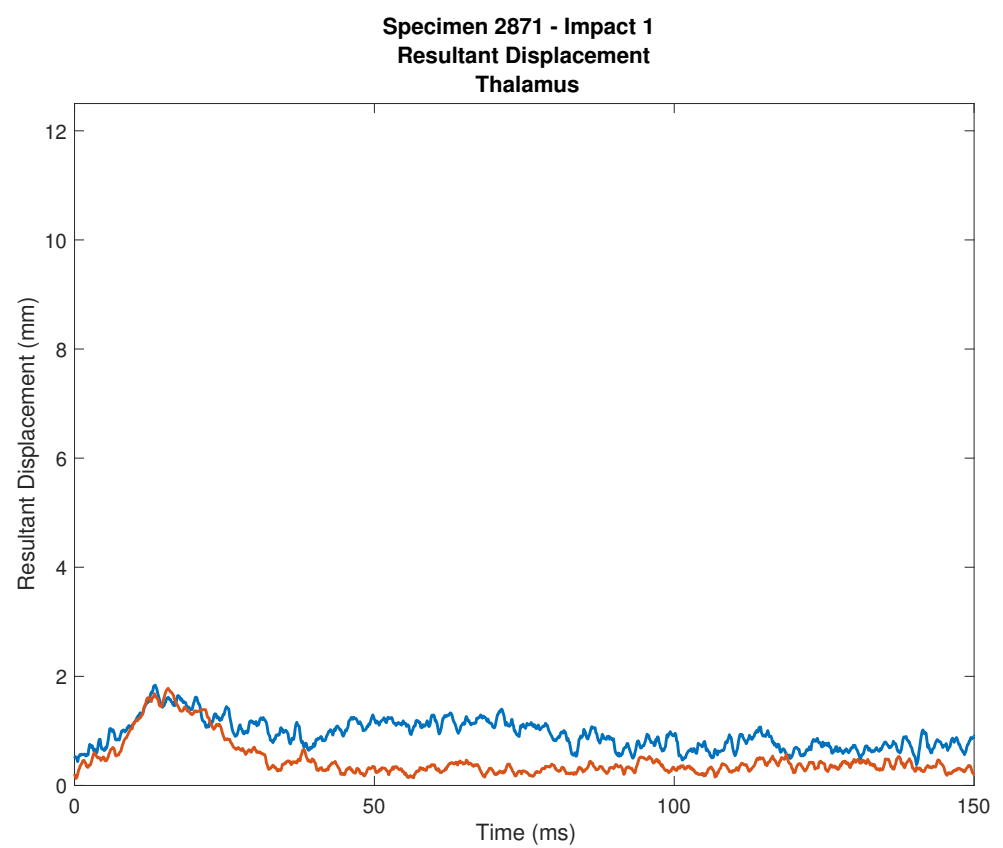

Figure 86: Displacement of thalamus - 2871-1 


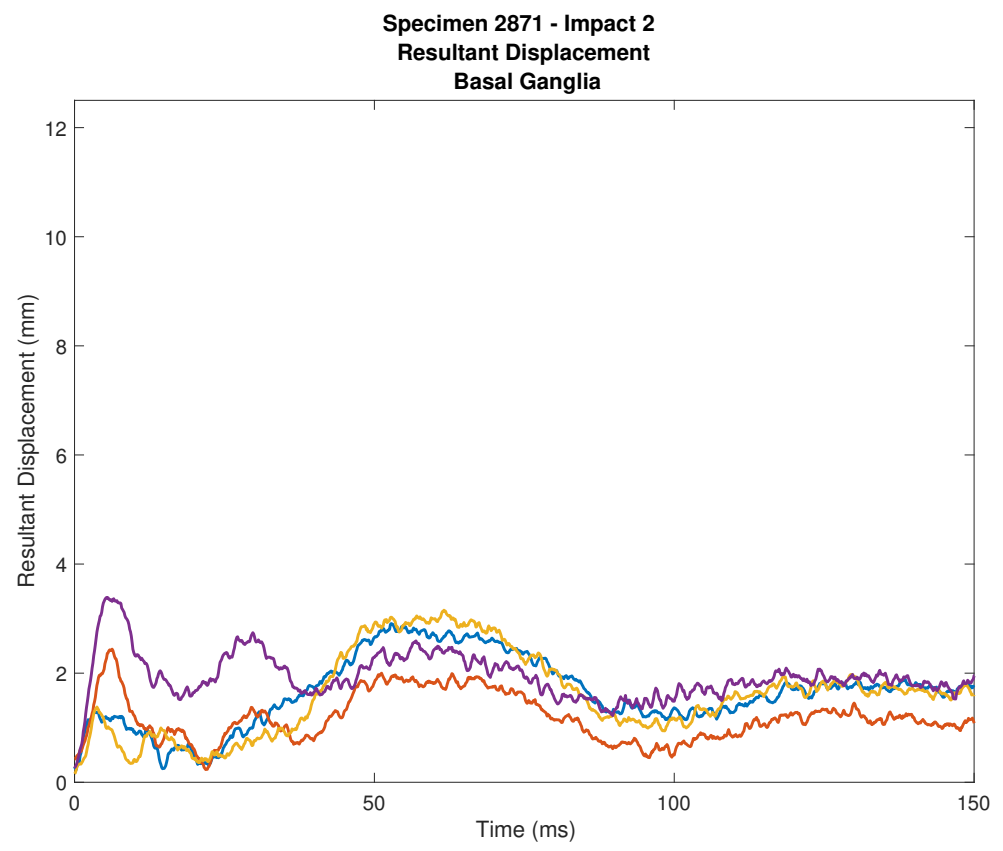

Figure 87: Displacement of basal ganglia - 2871-2

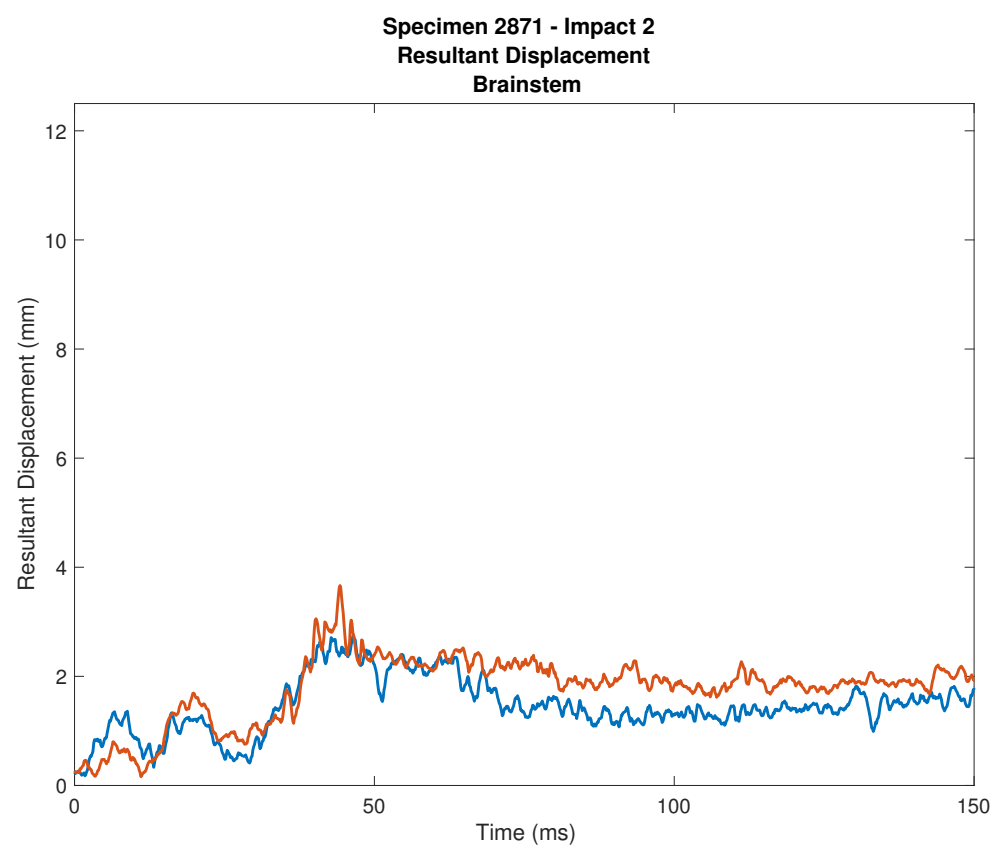

Figure 88: Displacement of brainstem - 2871-2 


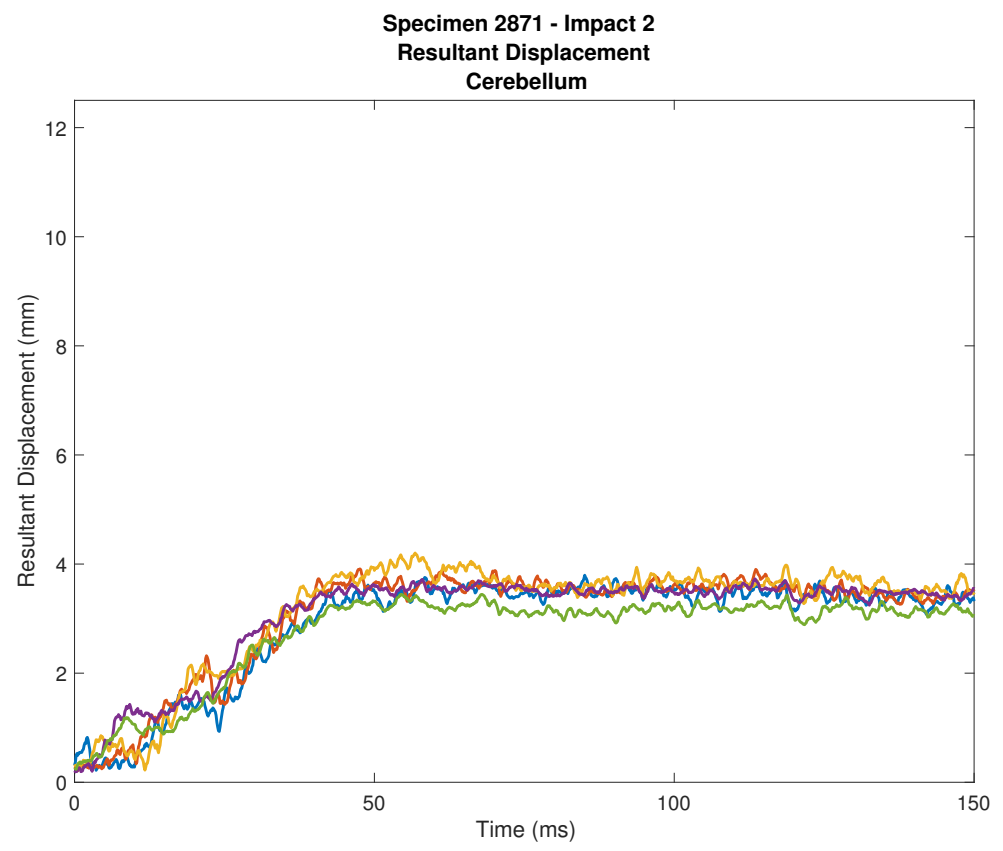

Figure 89: Displacement of cerebellum - 2871-2

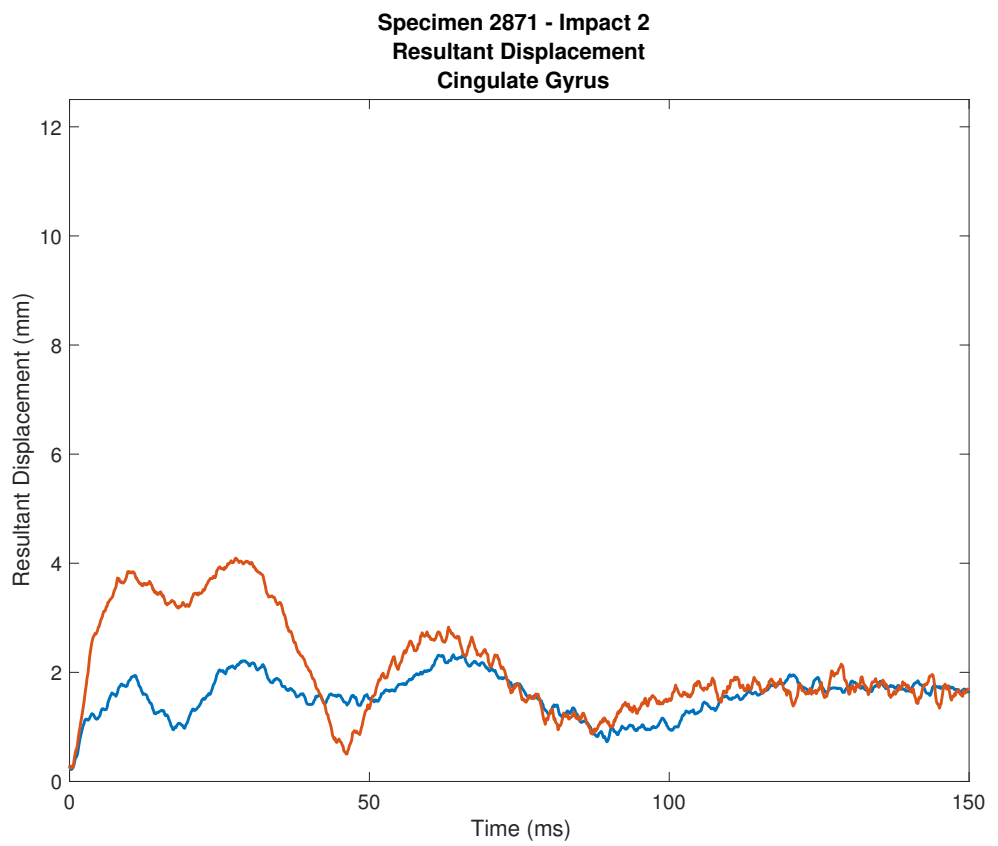

Figure 90: Displacement of cingulate gyrus - 2871-2 


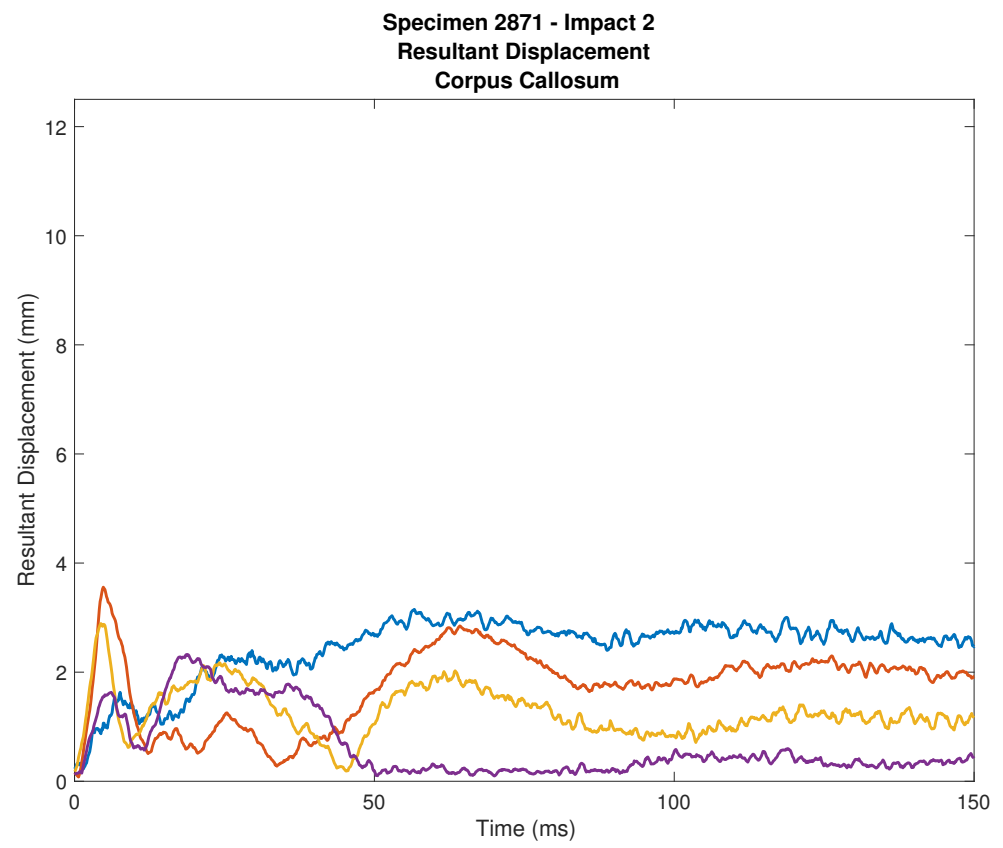

Figure 91: Displacement of corpus callosum - 2871-2

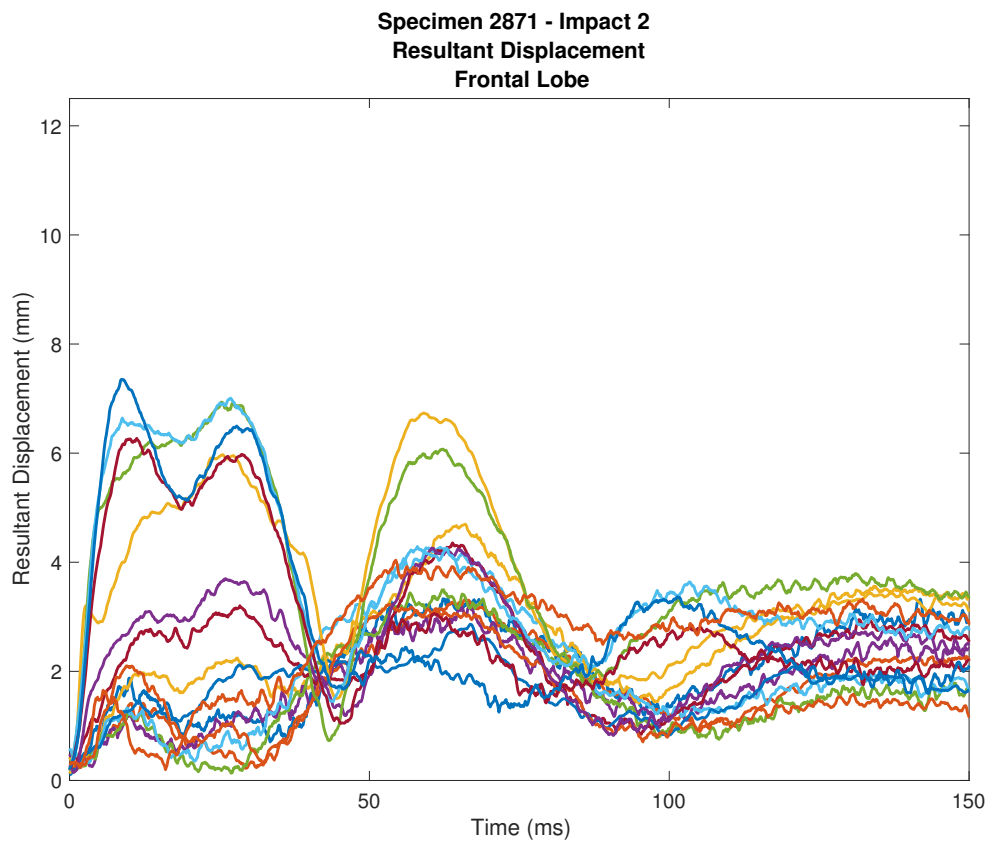

Figure 92: Displacement of frontal lobe - 2871-2 


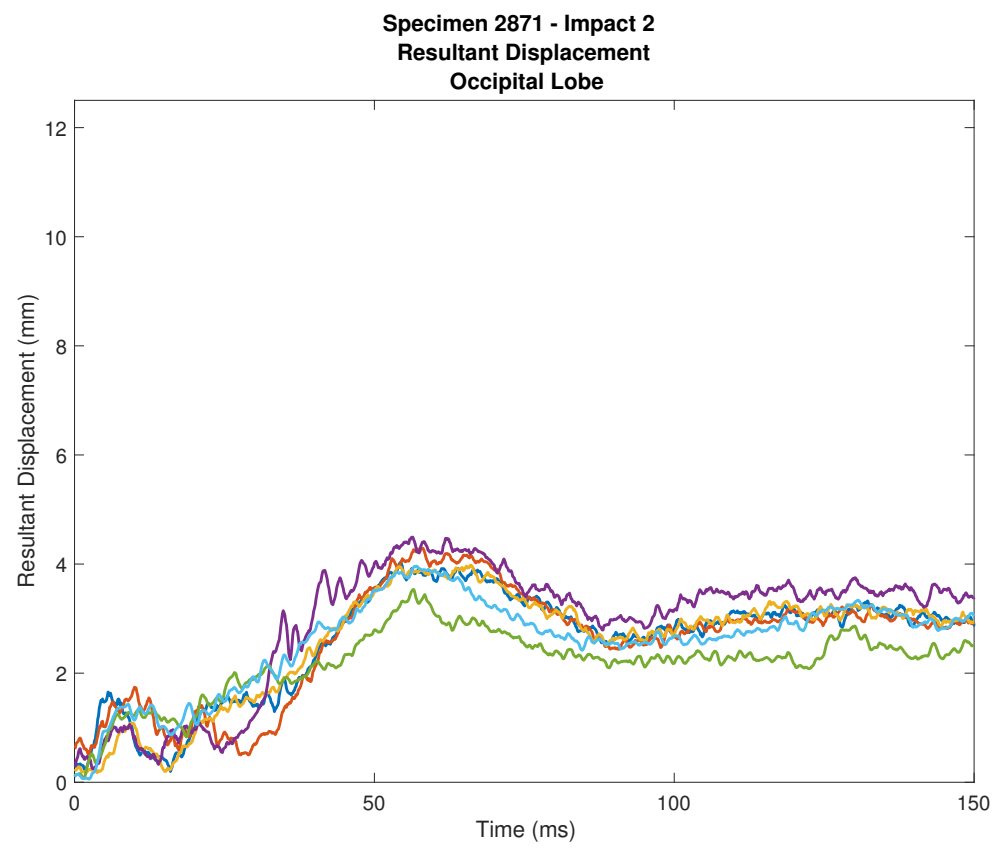

Figure 93: Displacement of occipital lobe - 2871-2

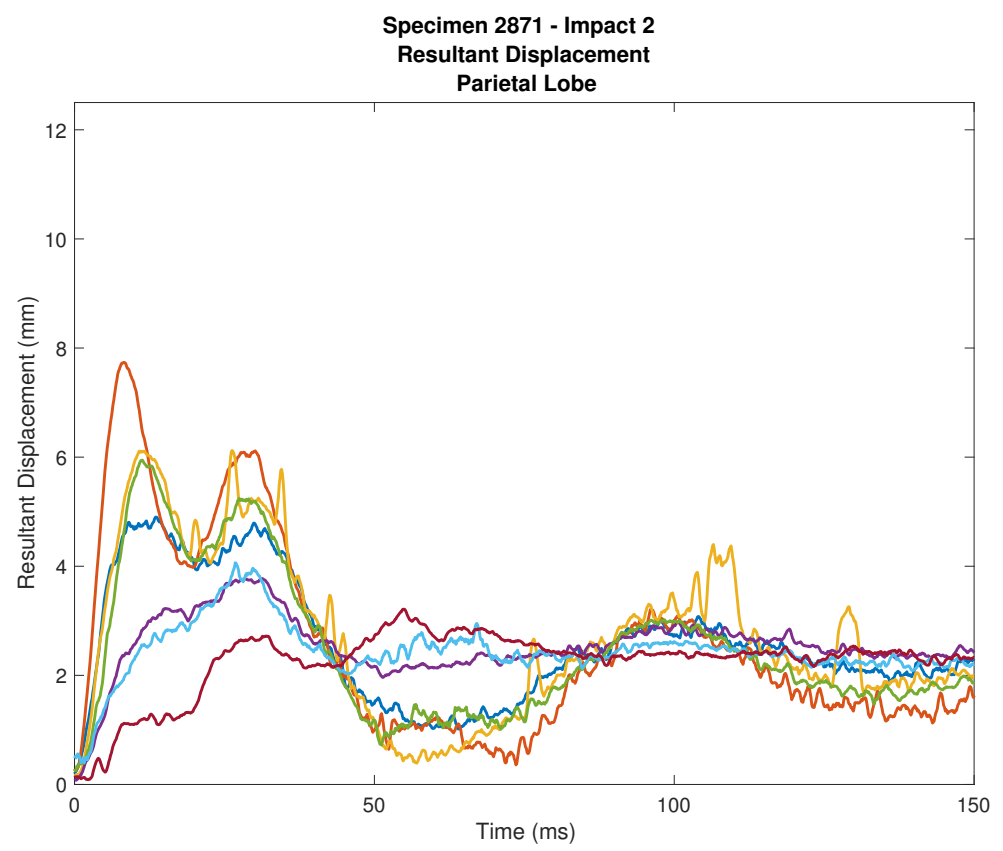

Figure 94: Displacement of parietal lobe - 2871-2 


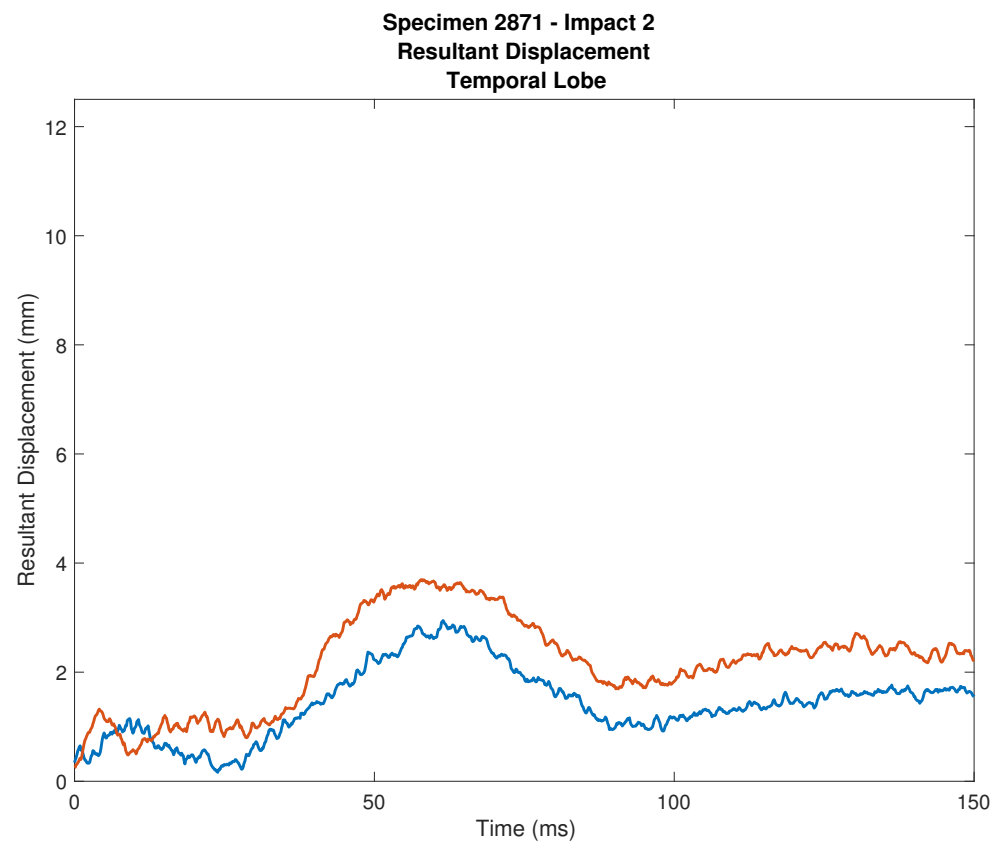

Figure 95: Displacement of temporal lobe - 2871-2

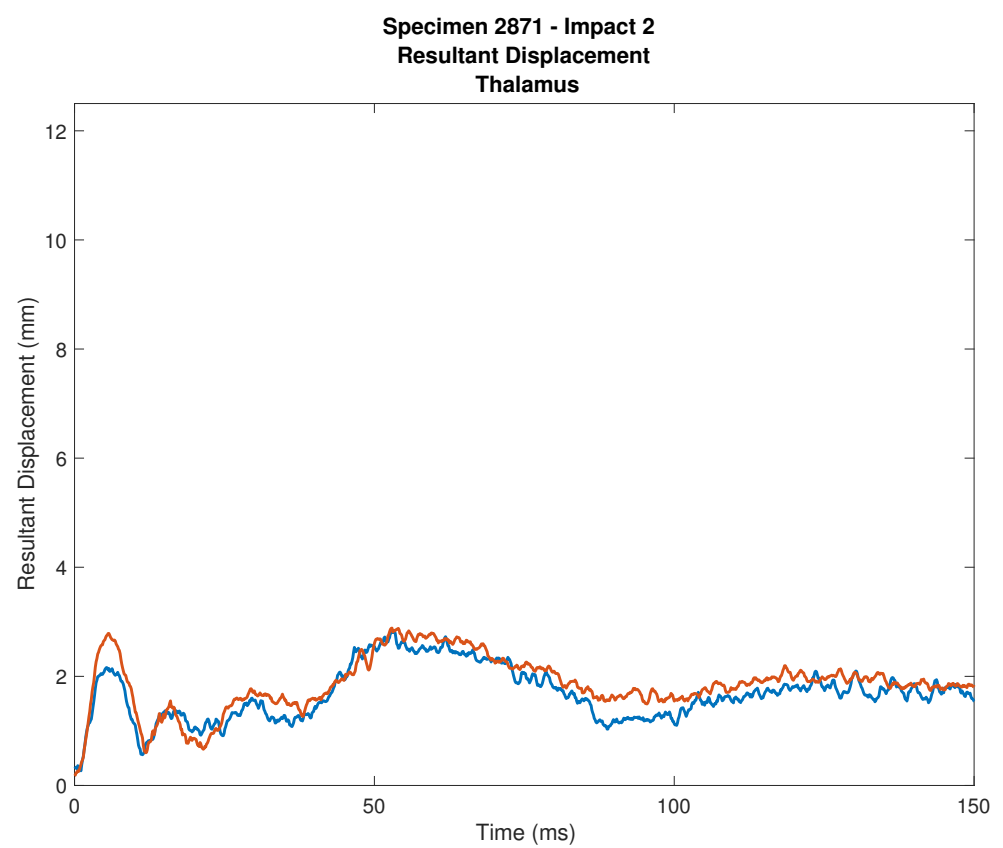

Figure 96: Displacement of thalamus - 2871-2 


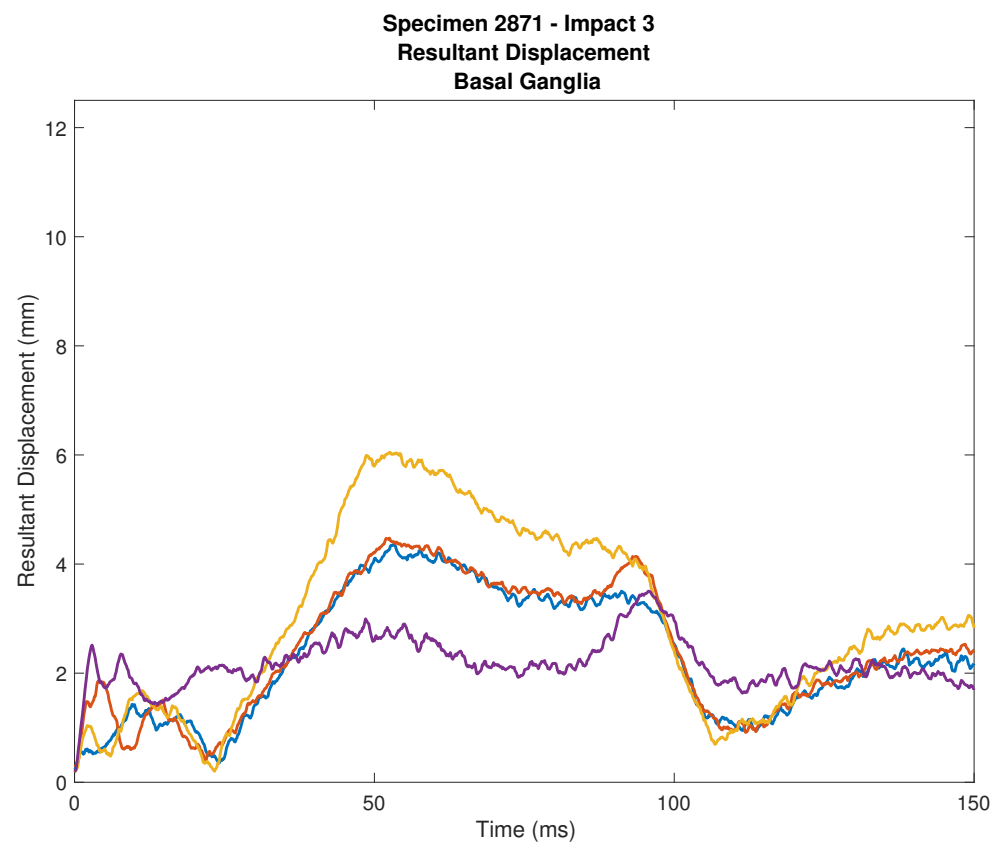

Figure 97: Displacement of basal ganglia - 2871-3

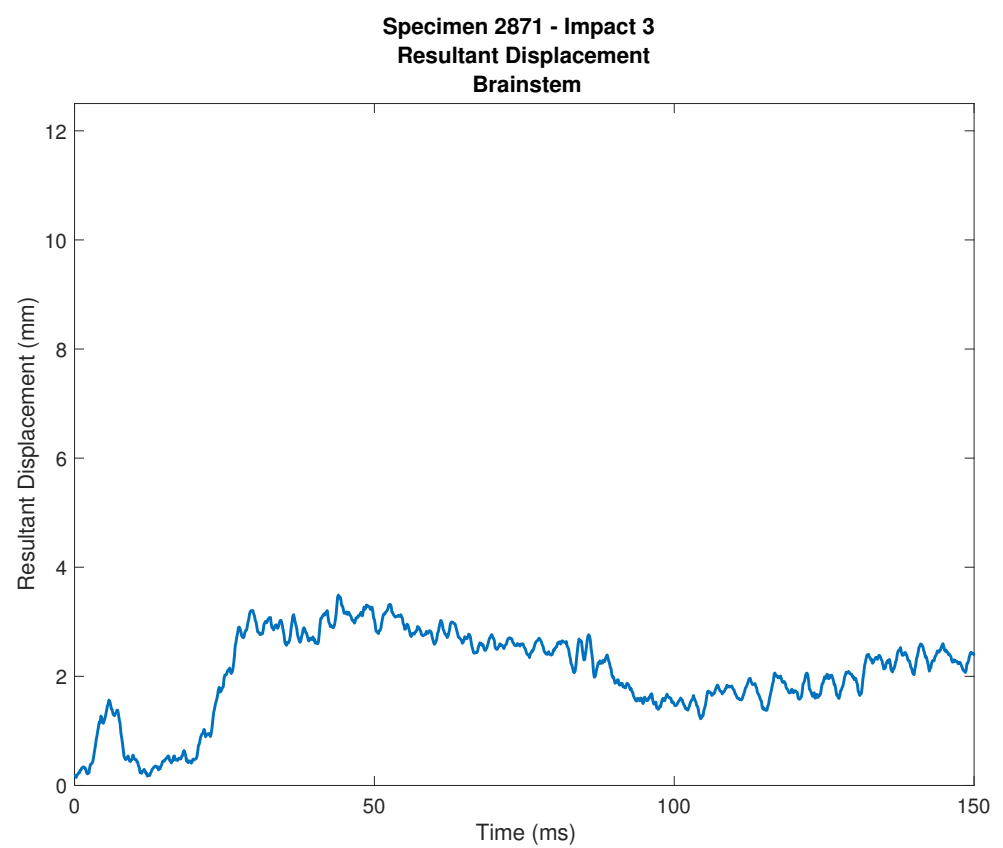

Figure 98: Displacement of brainstem - 2871-3 


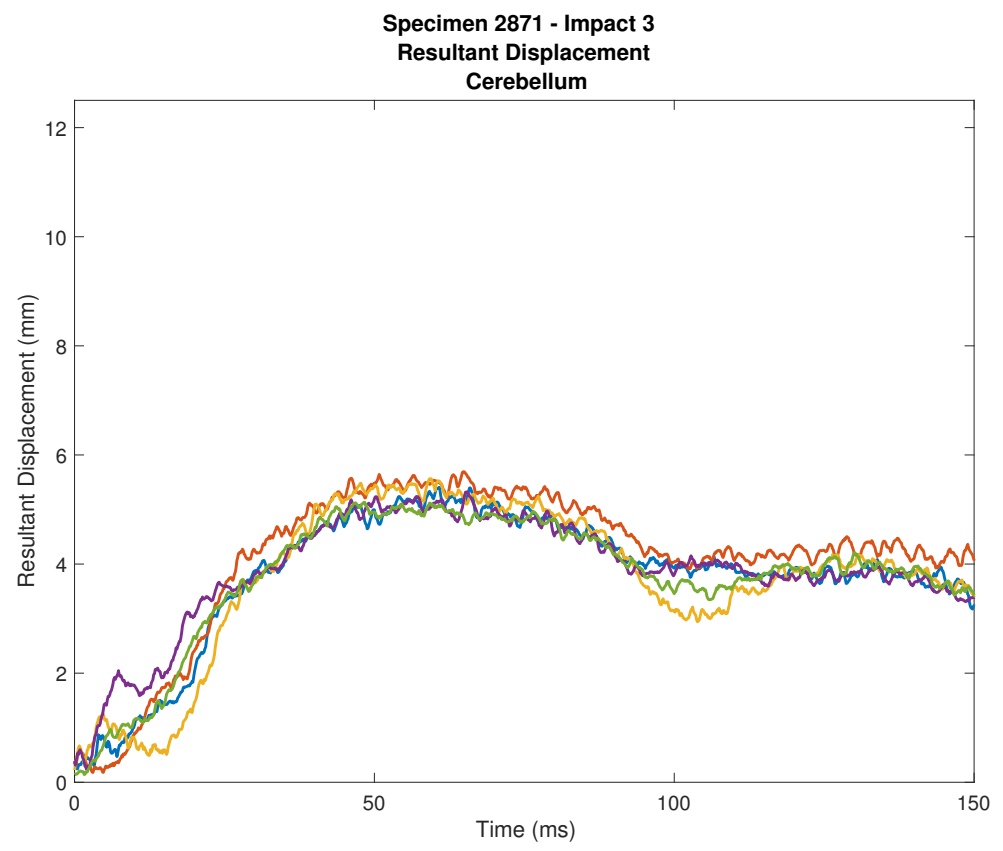

Figure 99: Displacement of cerebellum - 2871-3

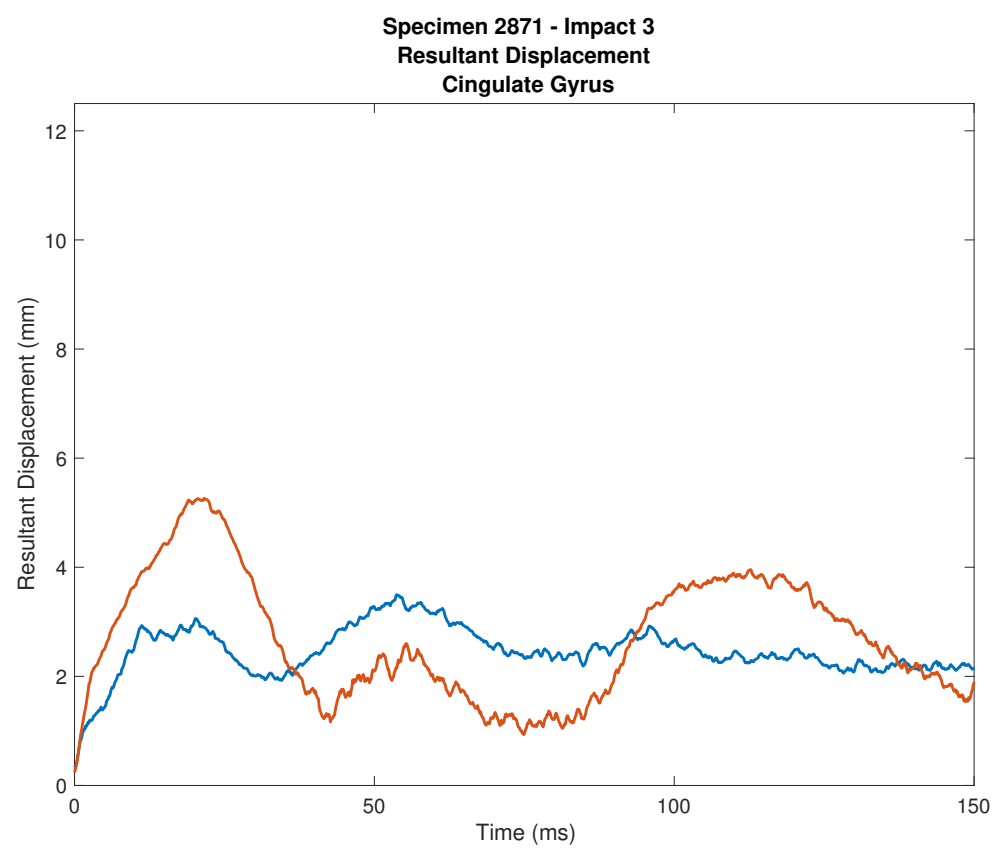

Figure 100: Displacement of cingulate gyrus - 2871-3 


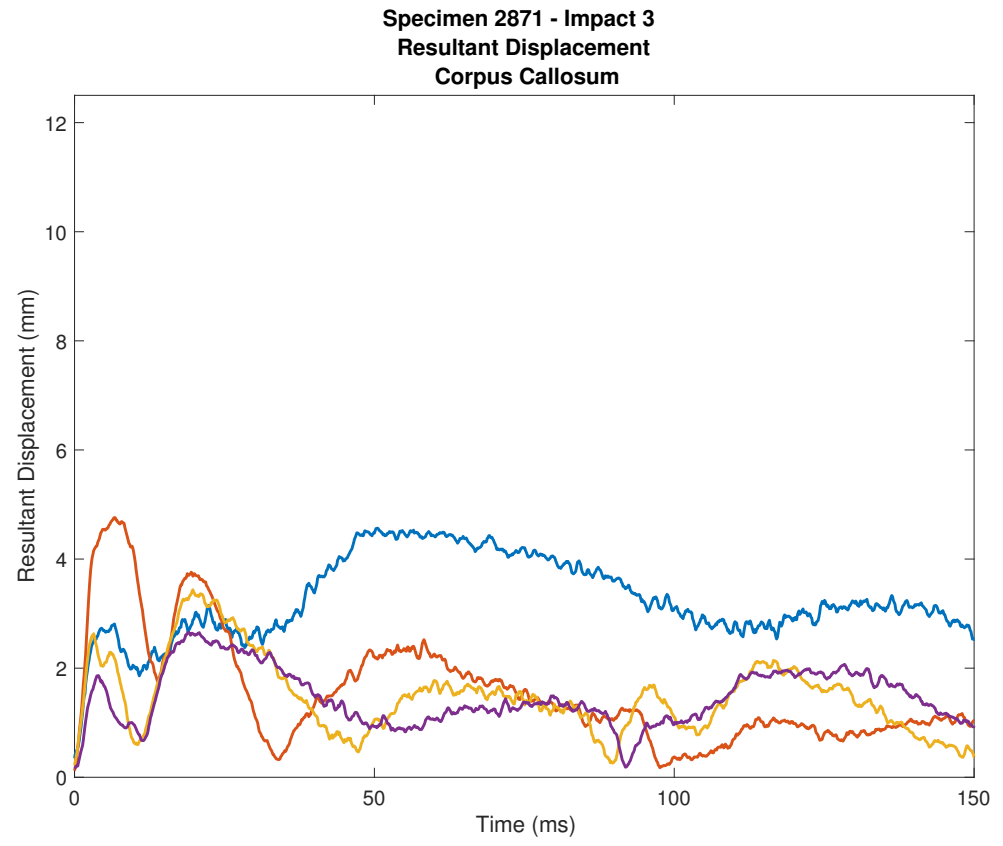

Figure 101: Displacement of corpus callosum - 2871-3

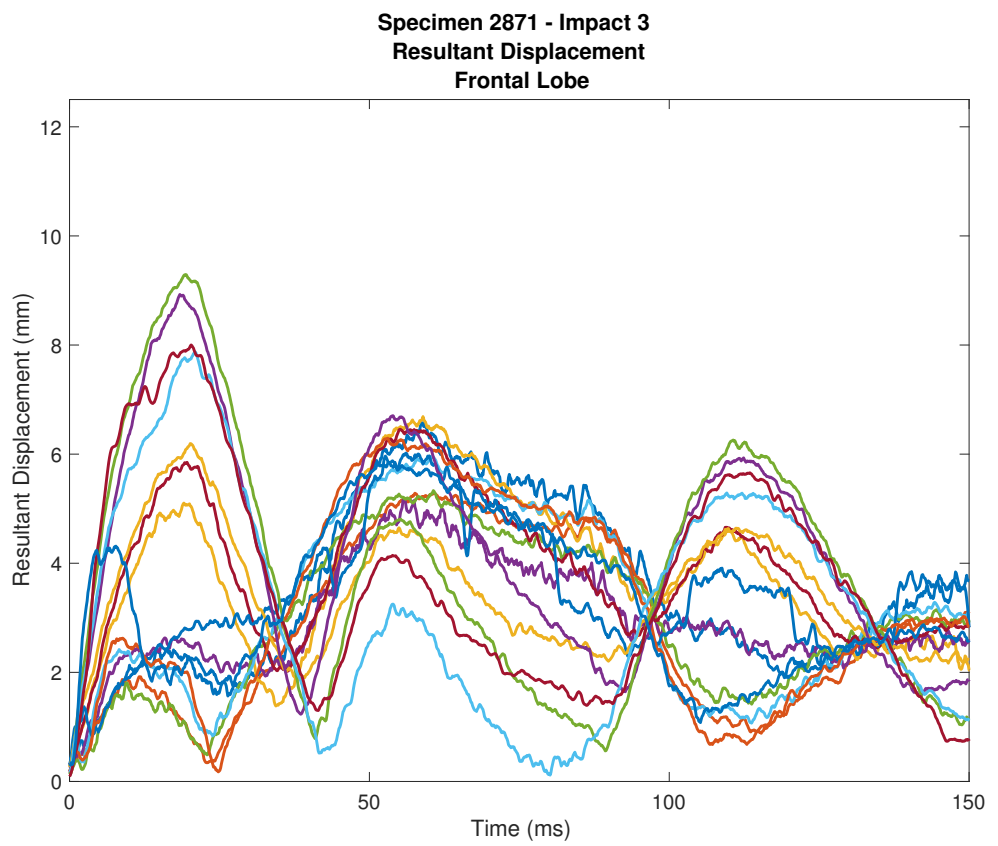

Figure 102: Displacement of frontal lobe - 2871-3 


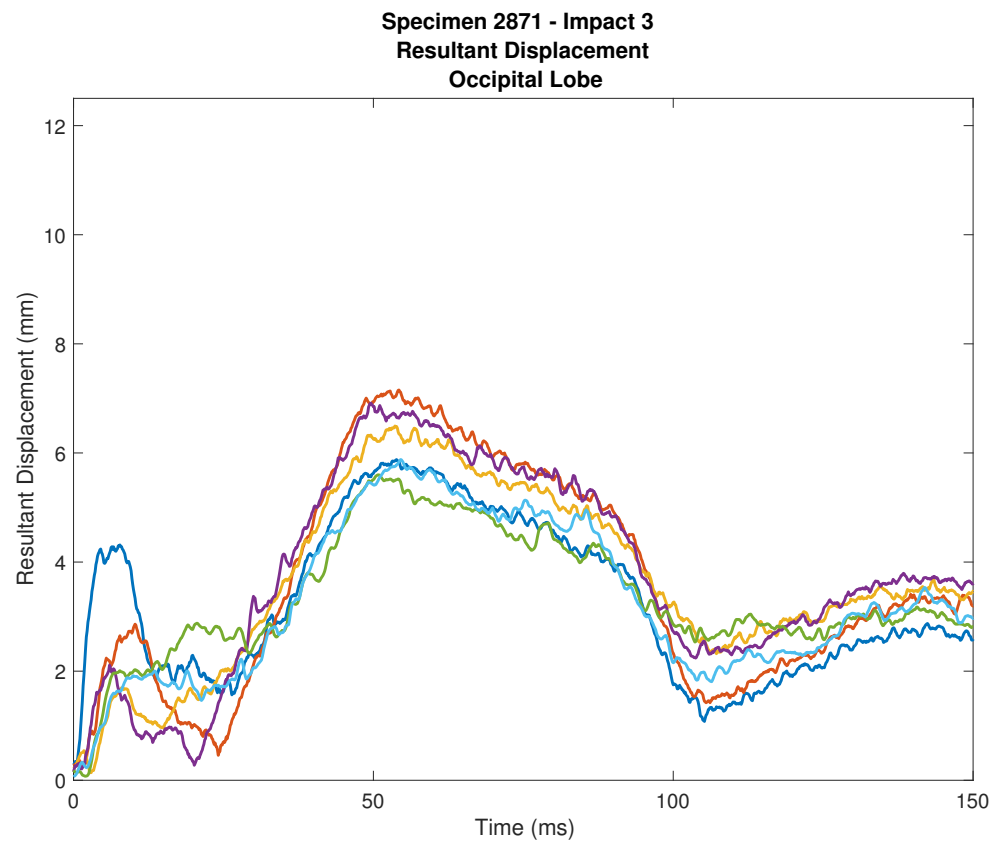

Figure 103: Displacement of occipital lobe - 2871-3

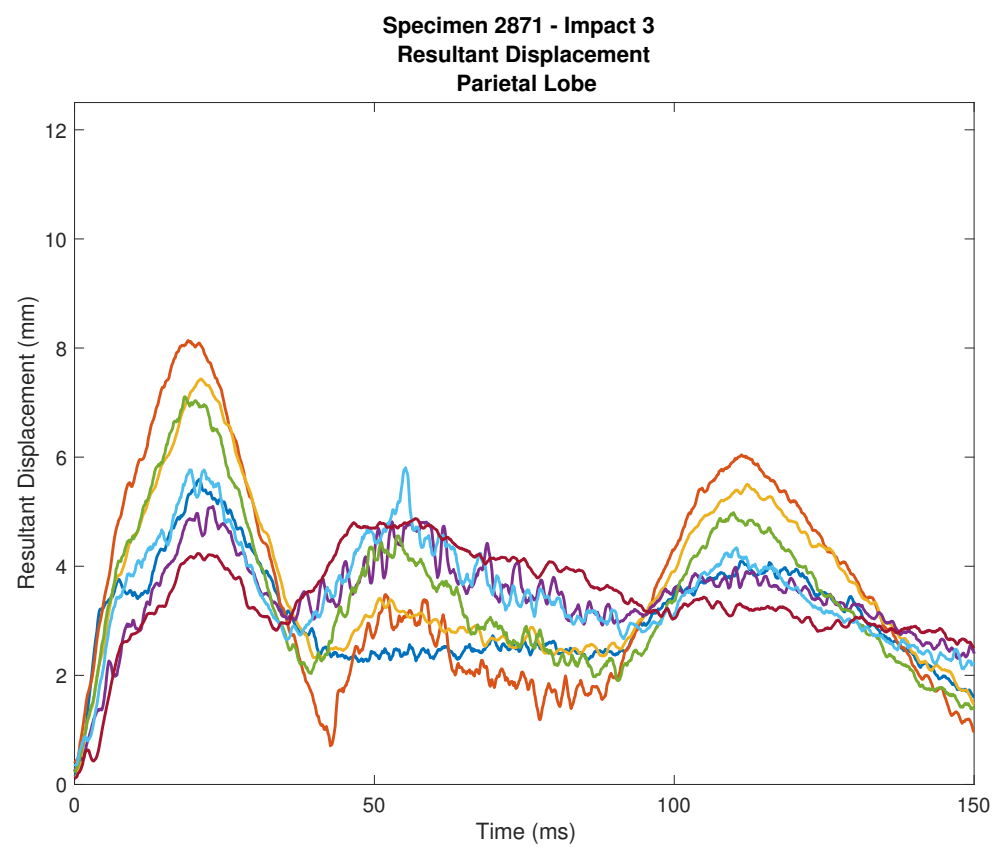

Figure 104: Displacement of parietal lobe - 2871-3 


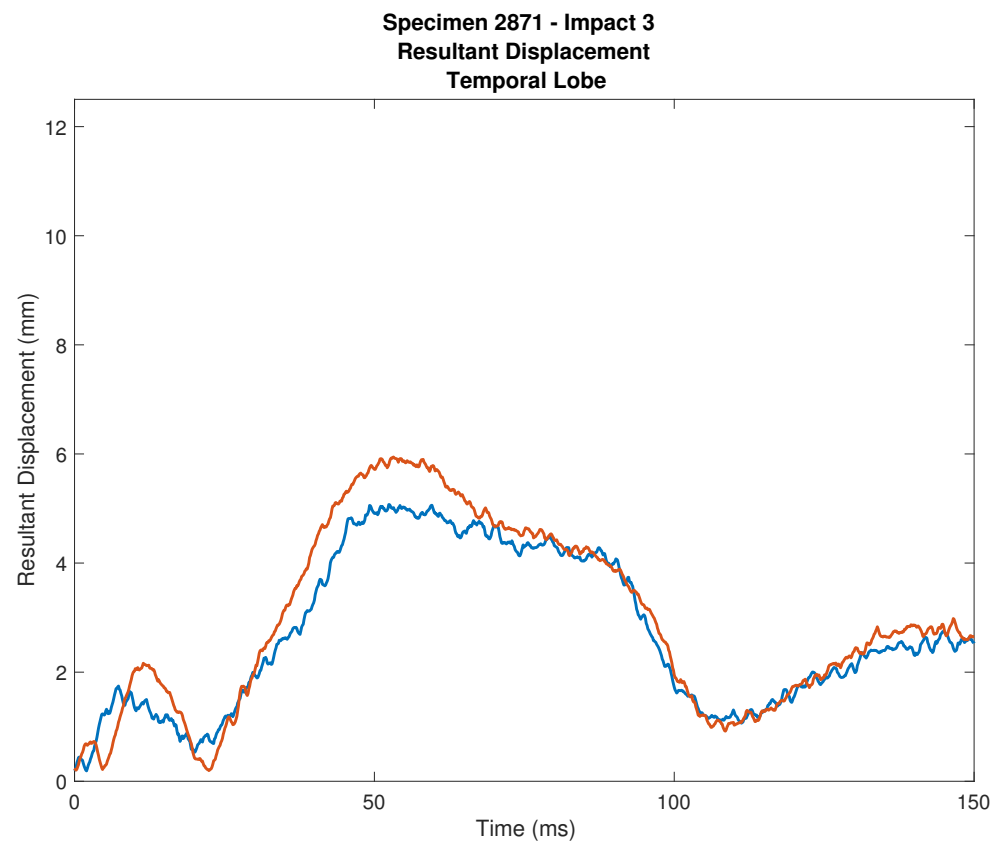

Figure 105: Displacement of temporal lobe - 2871-3

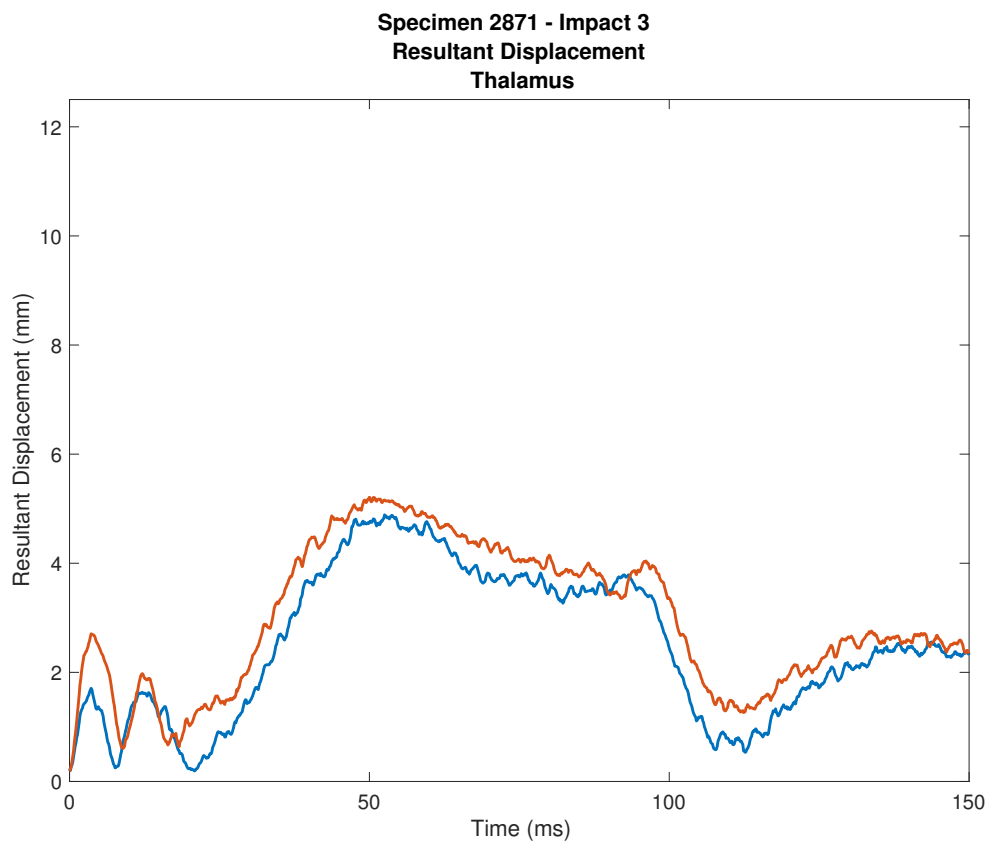

Figure 106: Displacement of thalamus - 2871-3 


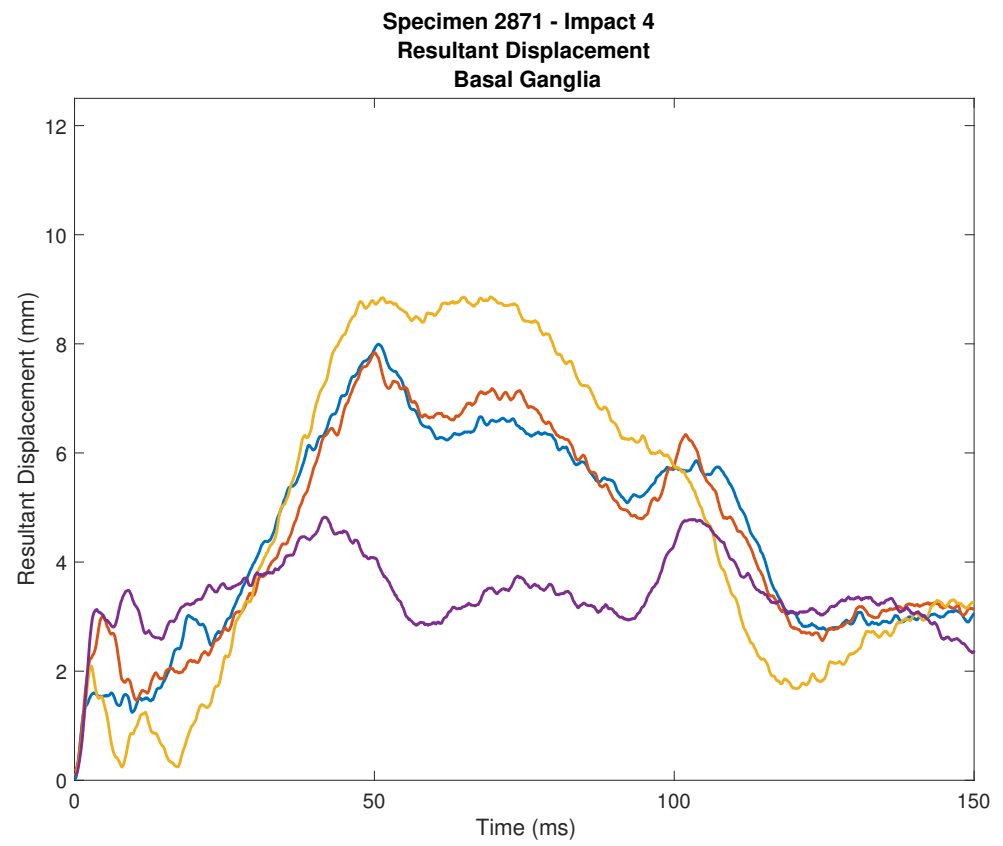

Figure 107: Displacement of basal ganglia - 2871-4

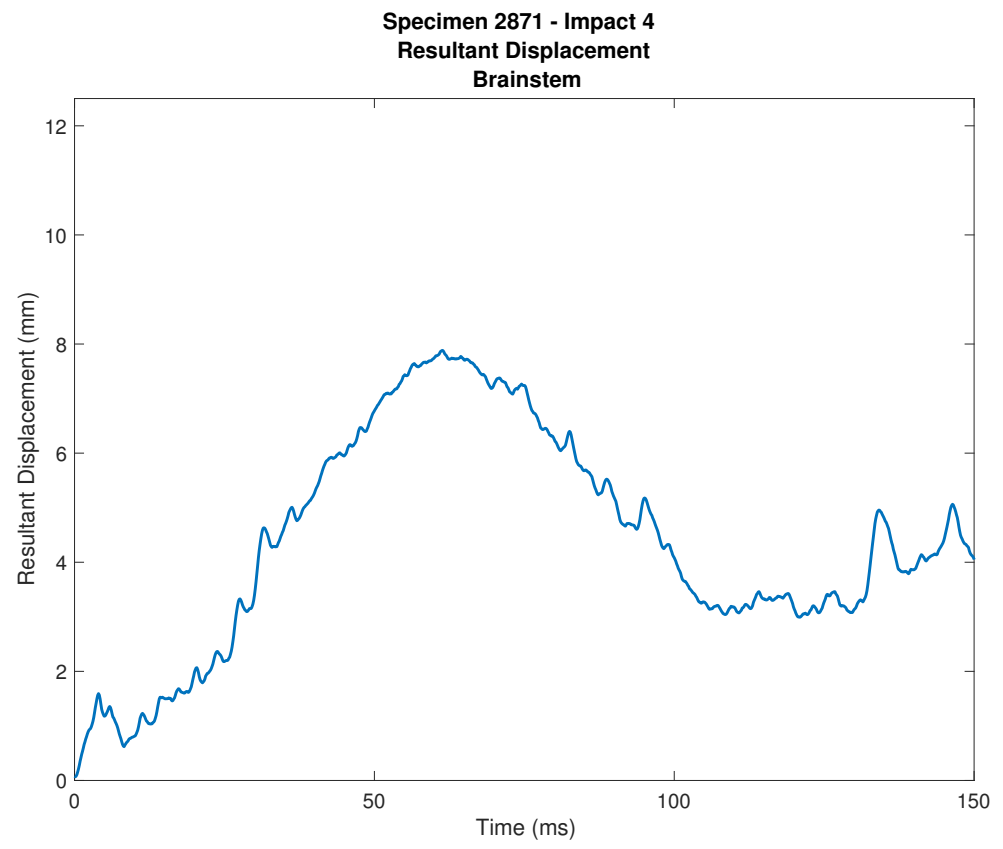

Figure 108: Displacement of brainstem - 2871-4 


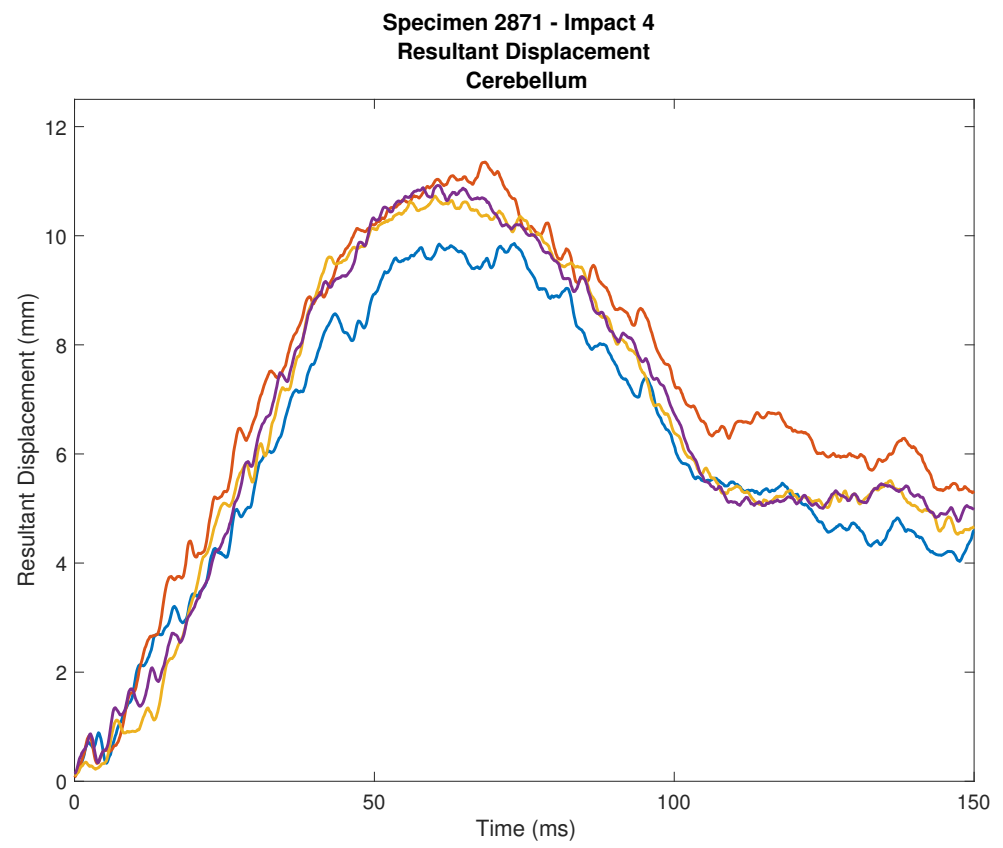

Figure 109: Displacement of cerebellum - 2871-4

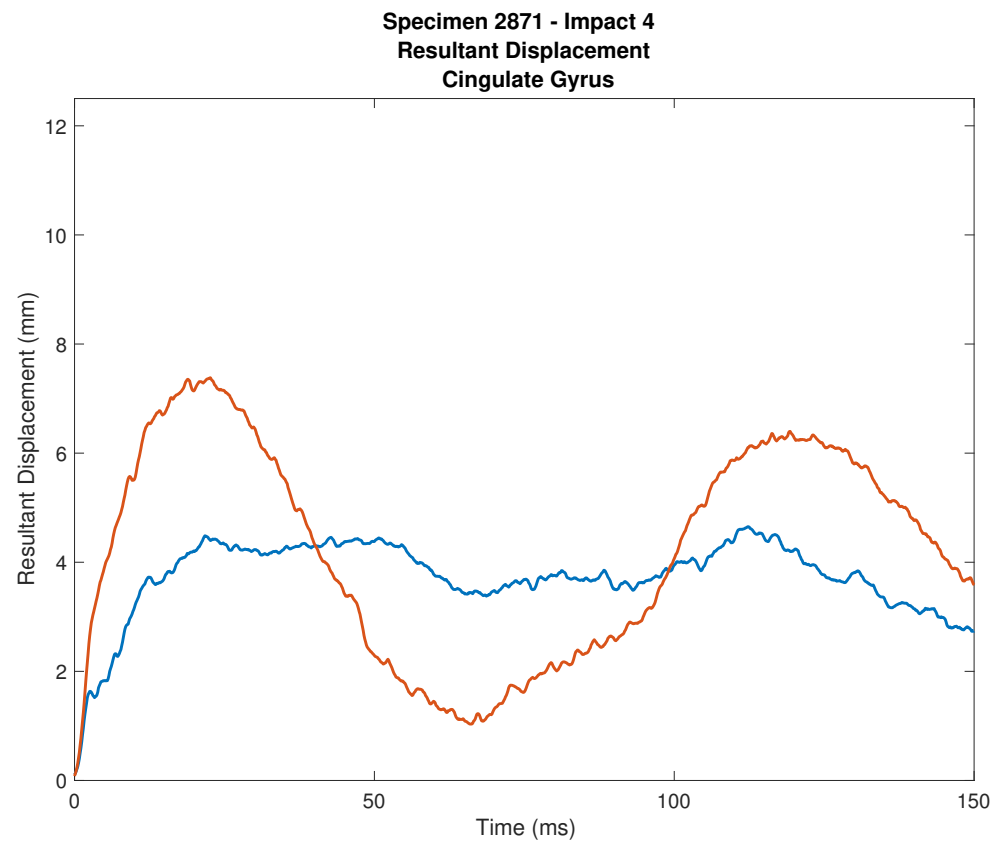

Figure 110: Displacement of cingulate gyrus - 2871-4 


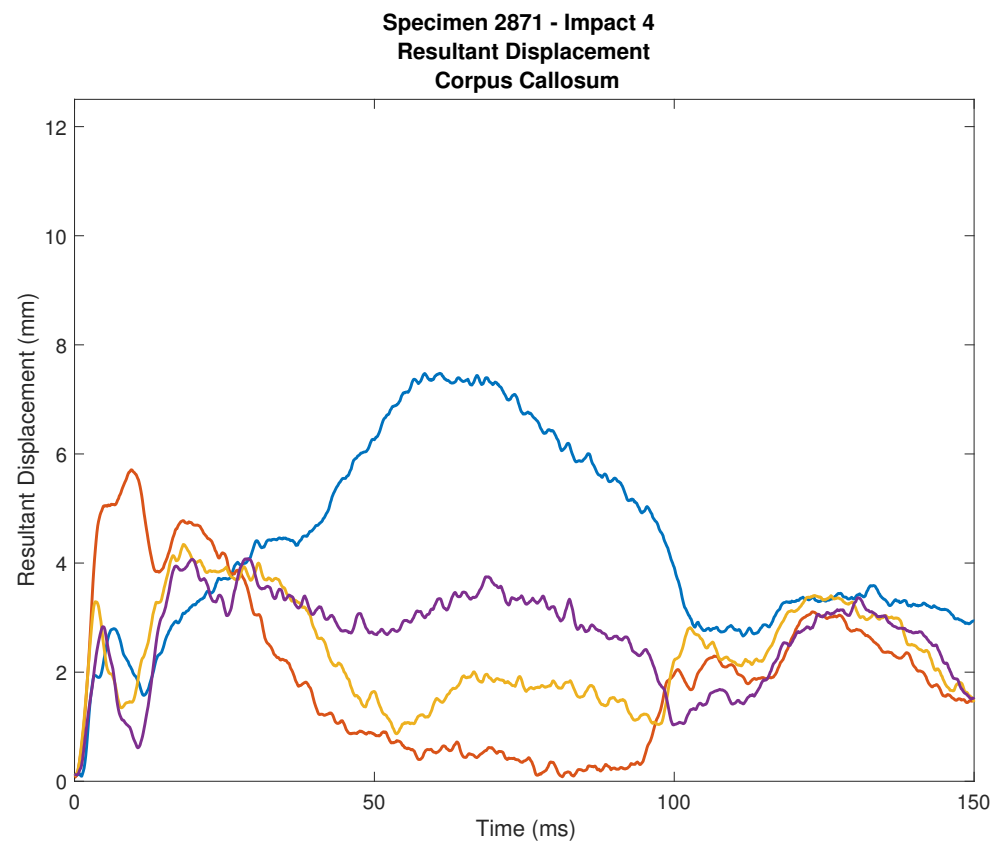

Figure 111: Displacement of corpus callosum - 2871-4

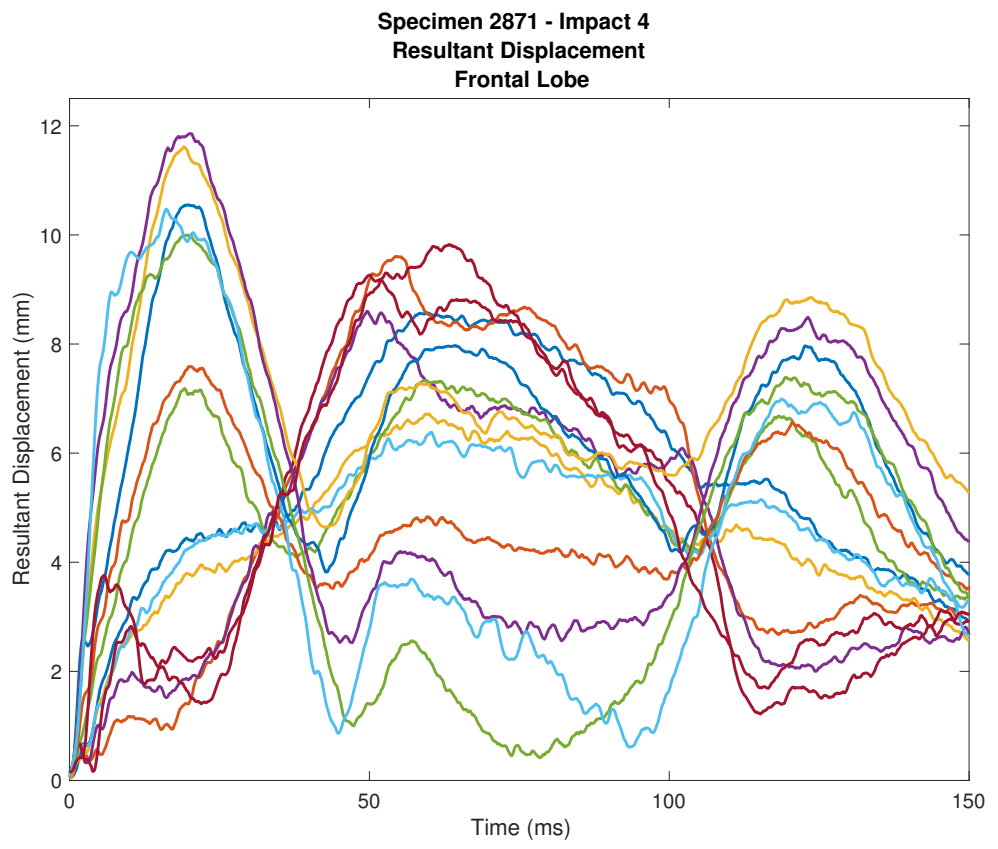

Figure 112: Displacement of frontal lobe - 2871-4 


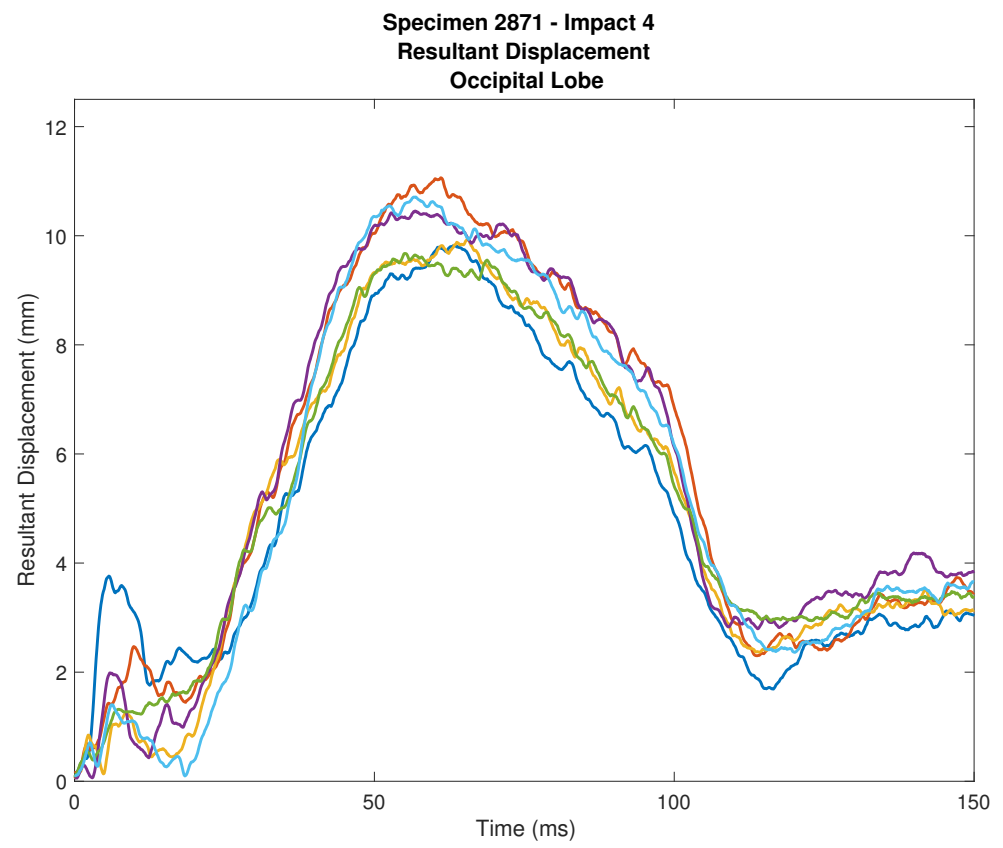

Figure 113: Displacement of occipital lobe - 2871-4

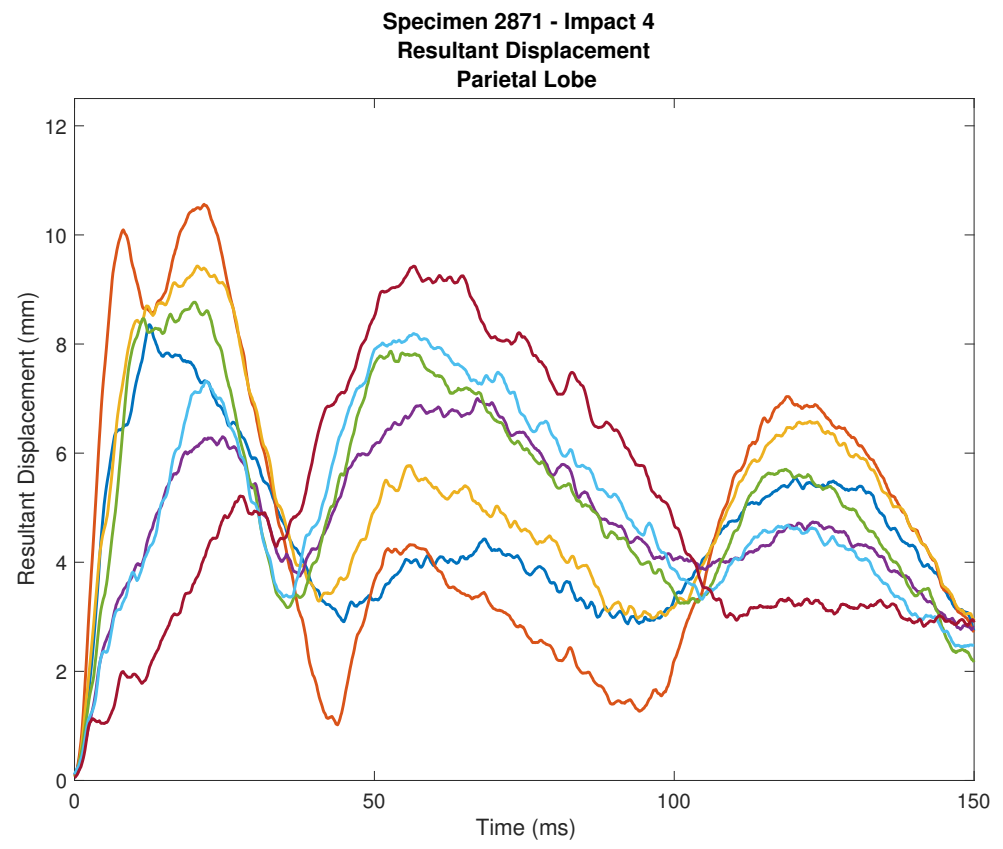

Figure 114: Displacement of parietal lobe - 2871-4 


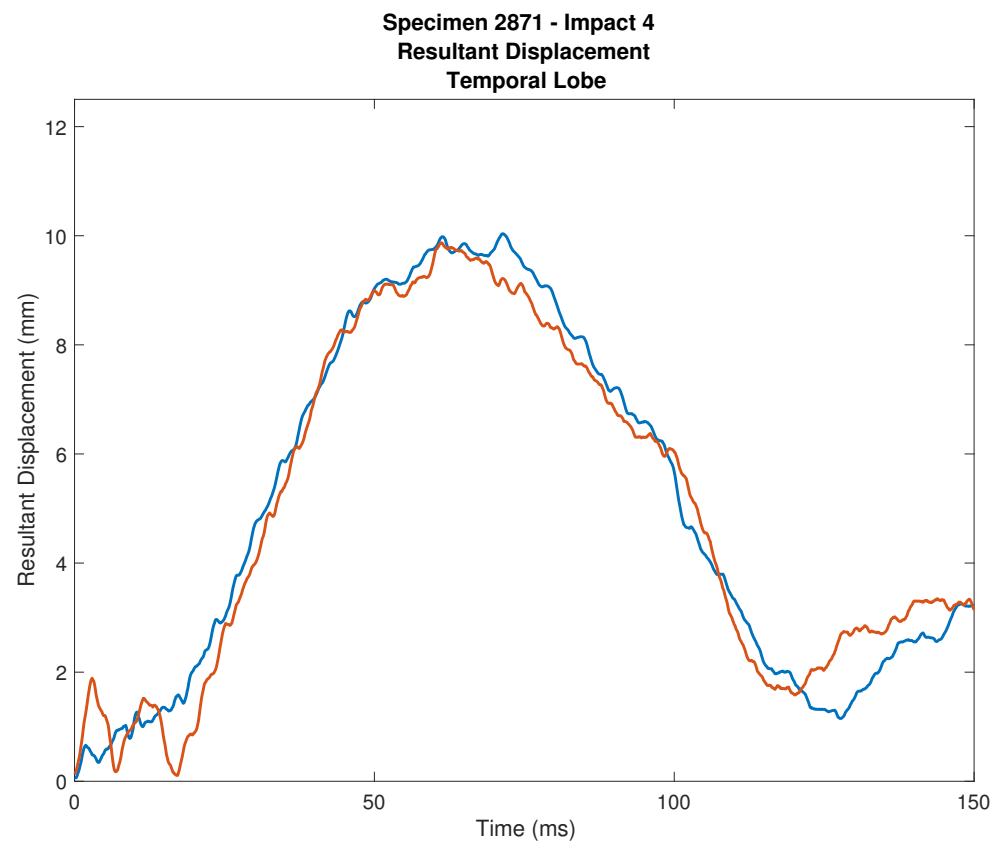

Figure 115: Displacement of temporal lobe - 2871-4

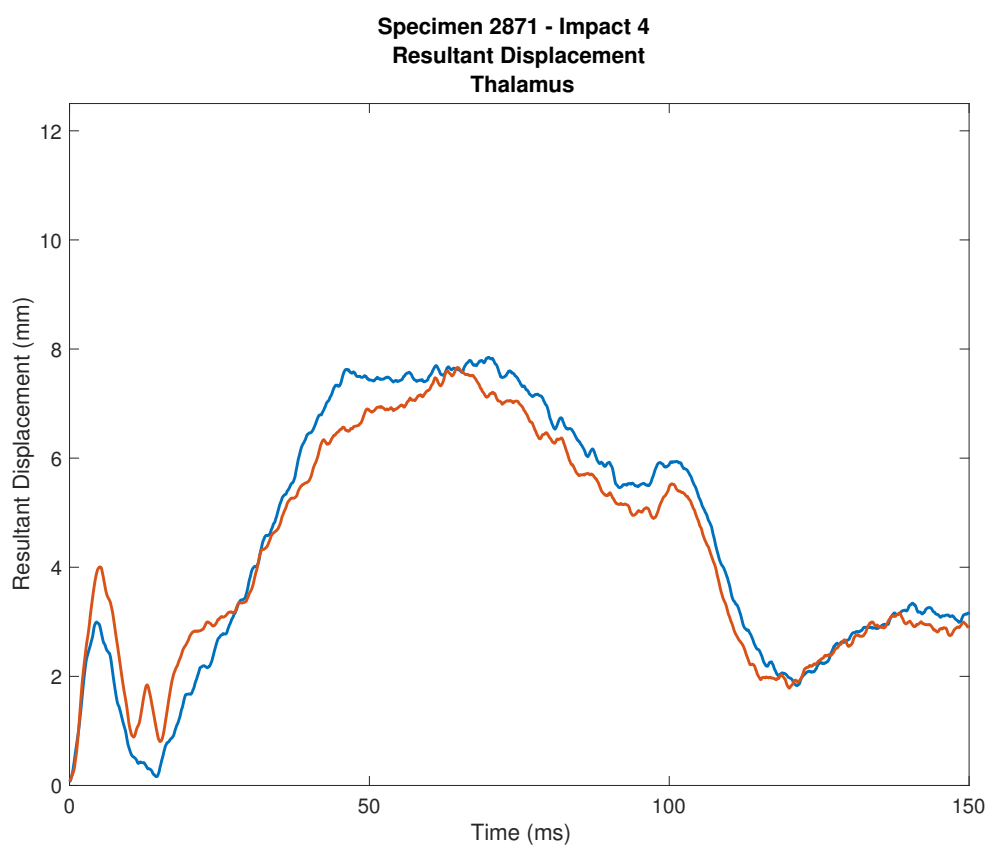

Figure 116: Displacement of thalamus - 2871-4 


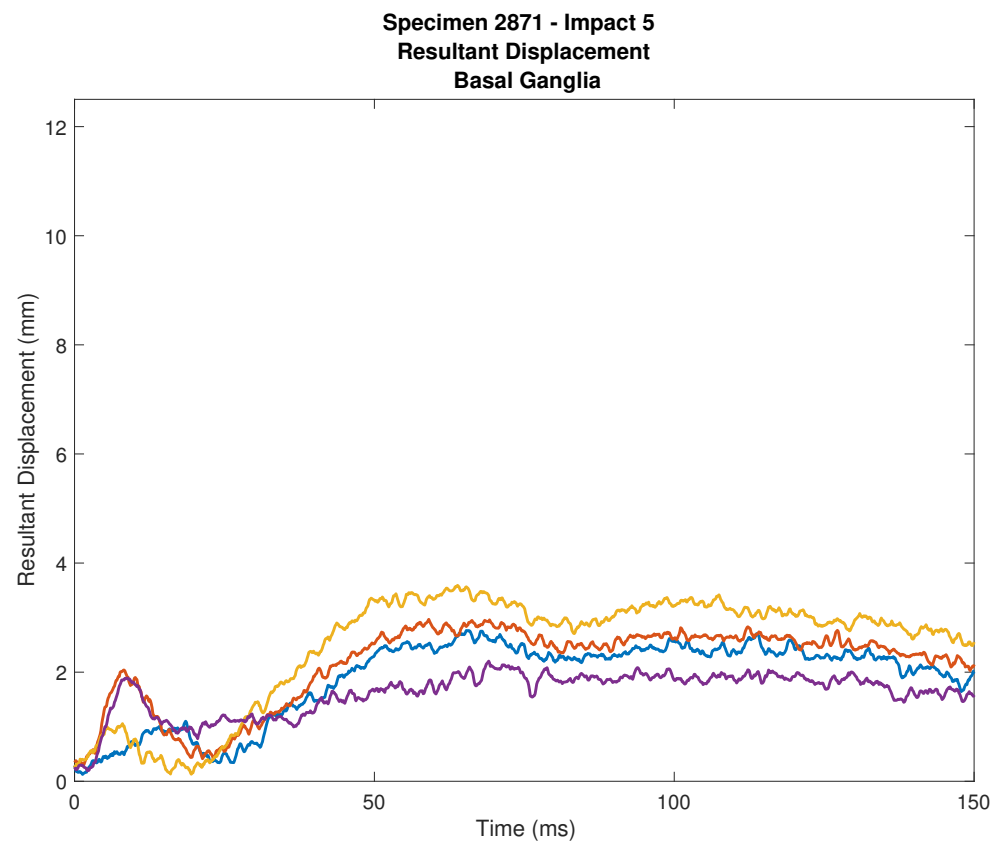

Figure 117: Displacement of basal ganglia - 2871-5

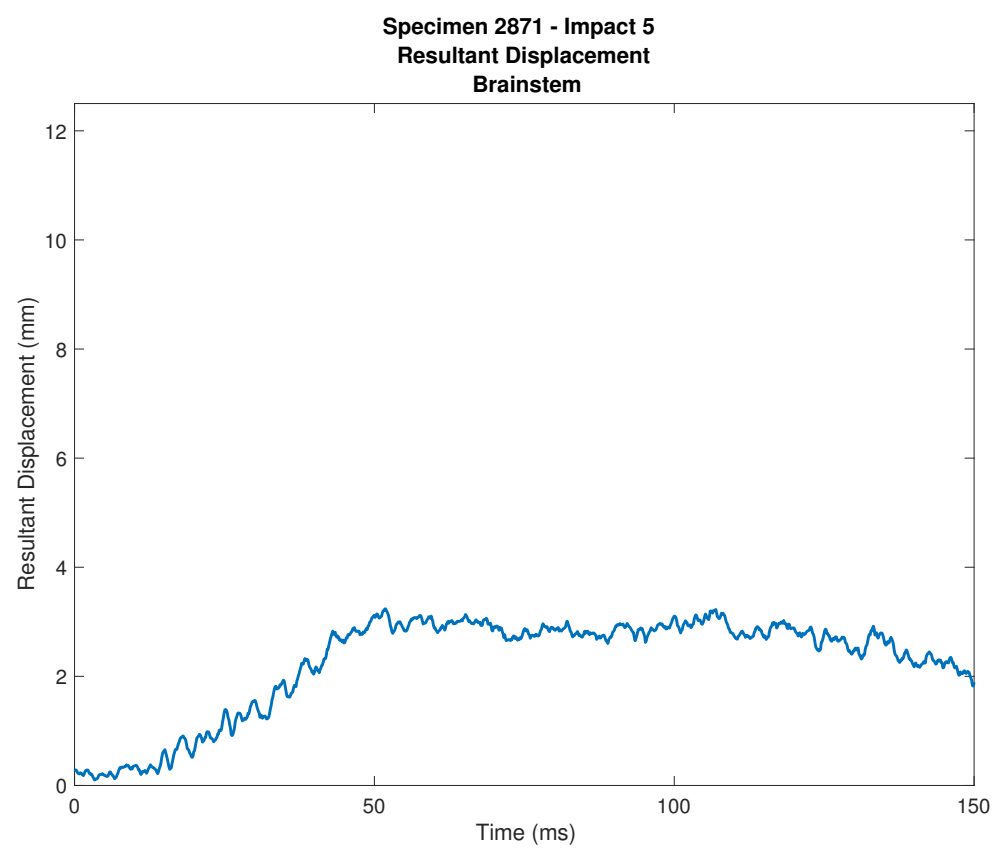

Figure 118: Displacement of brainstem - 2871-5 


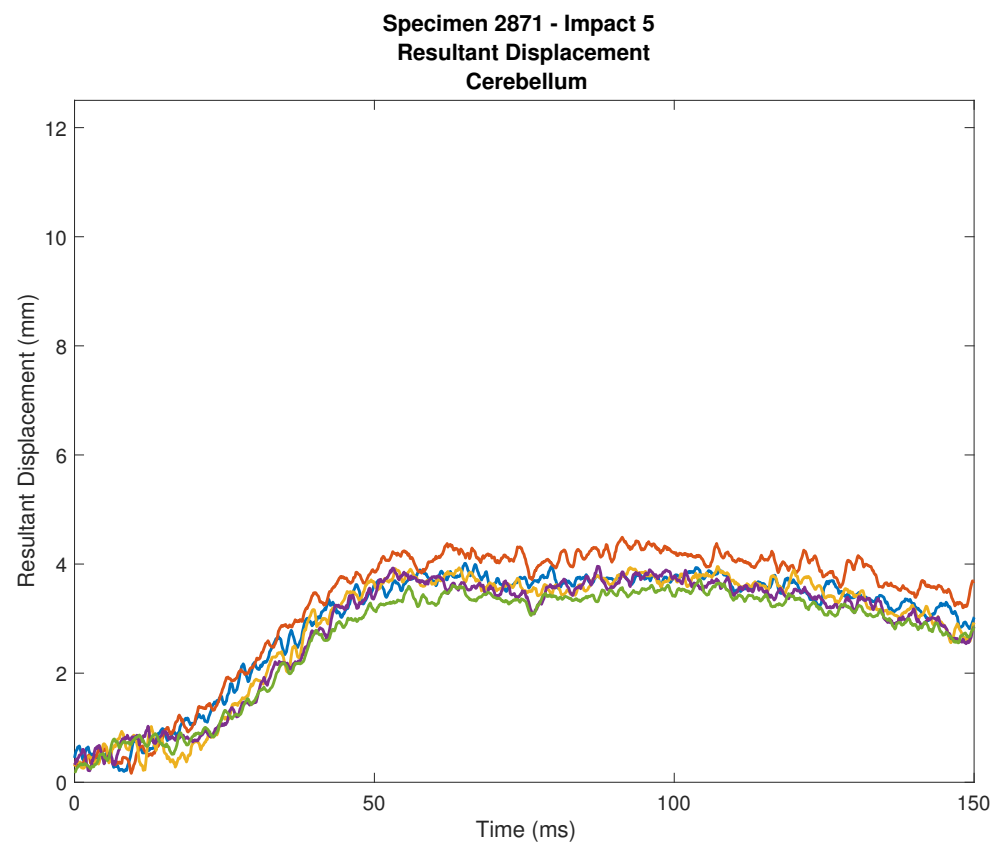

Figure 119: Displacement of cerebellum - 2871-5

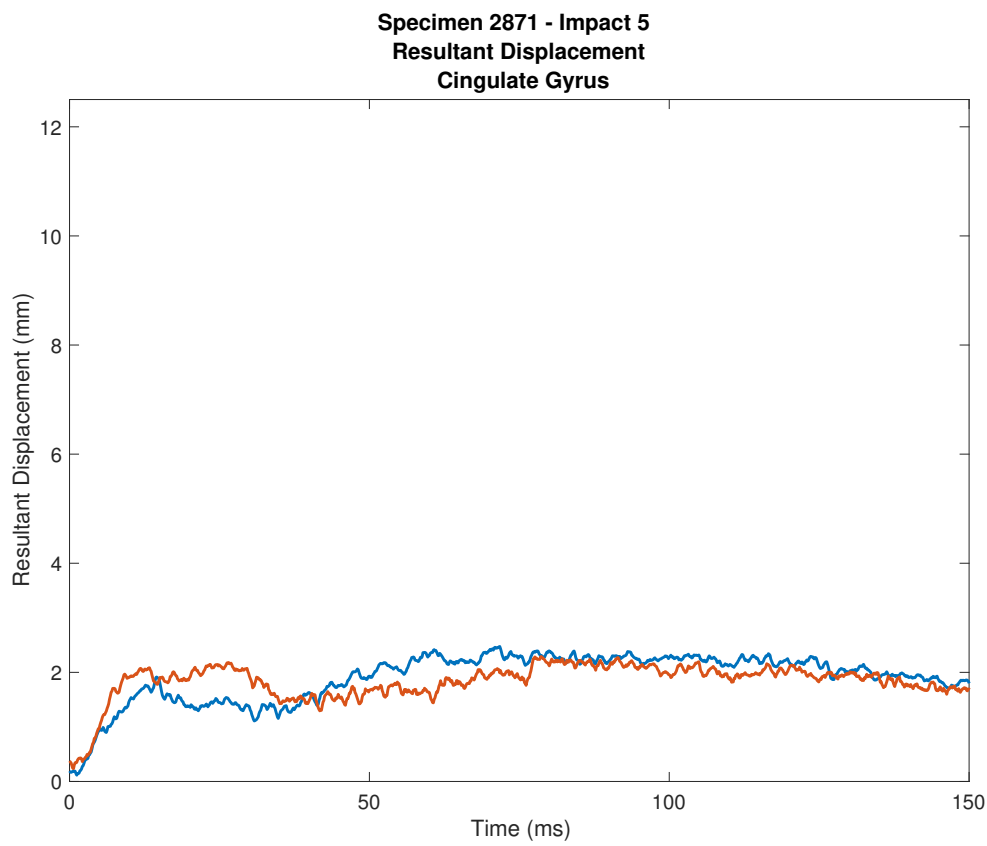

Figure 120: Displacement of cingulate gyrus - 2871-5 


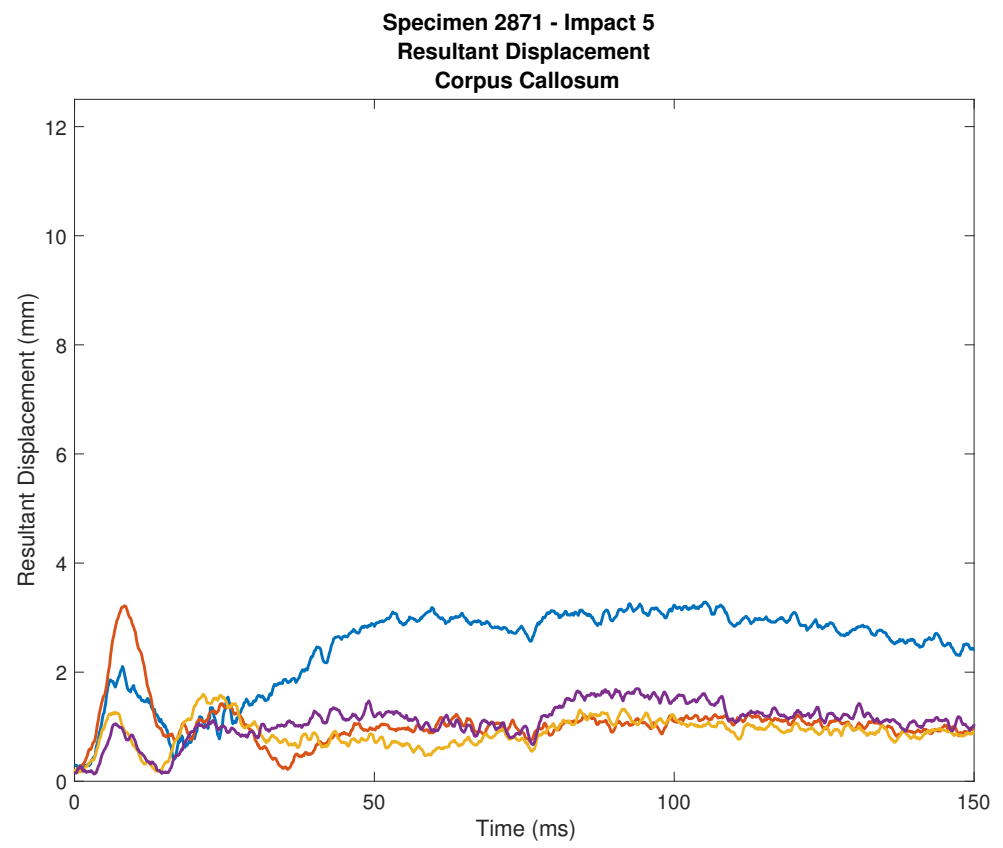

Figure 121: Displacement of corpus callosum - 2871-5

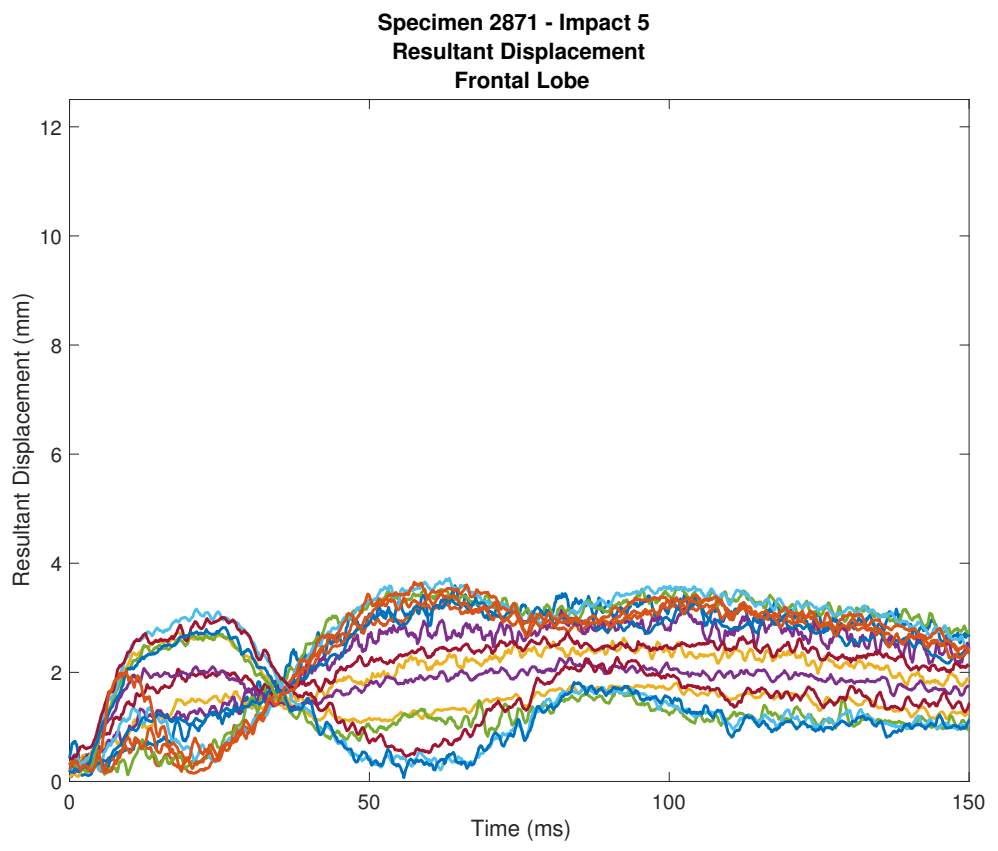

Figure 122: Displacement of frontal lobe - 2871-5 


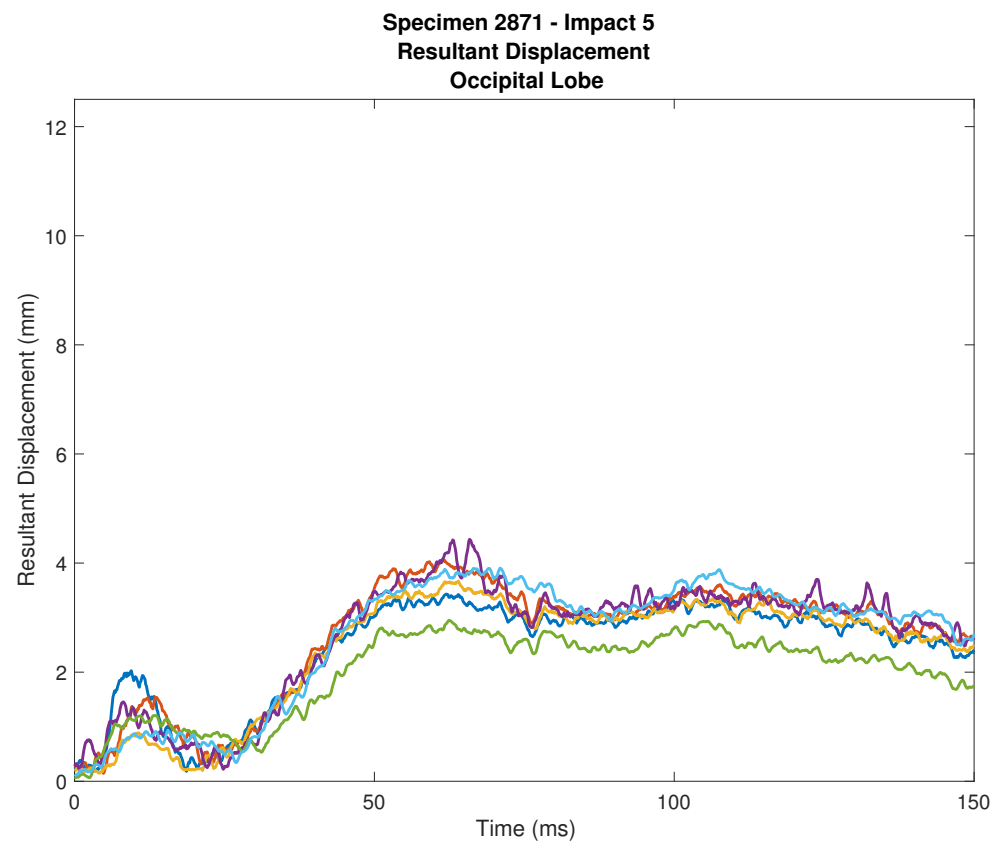

Figure 123: Displacement of occipital lobe - 2871-5

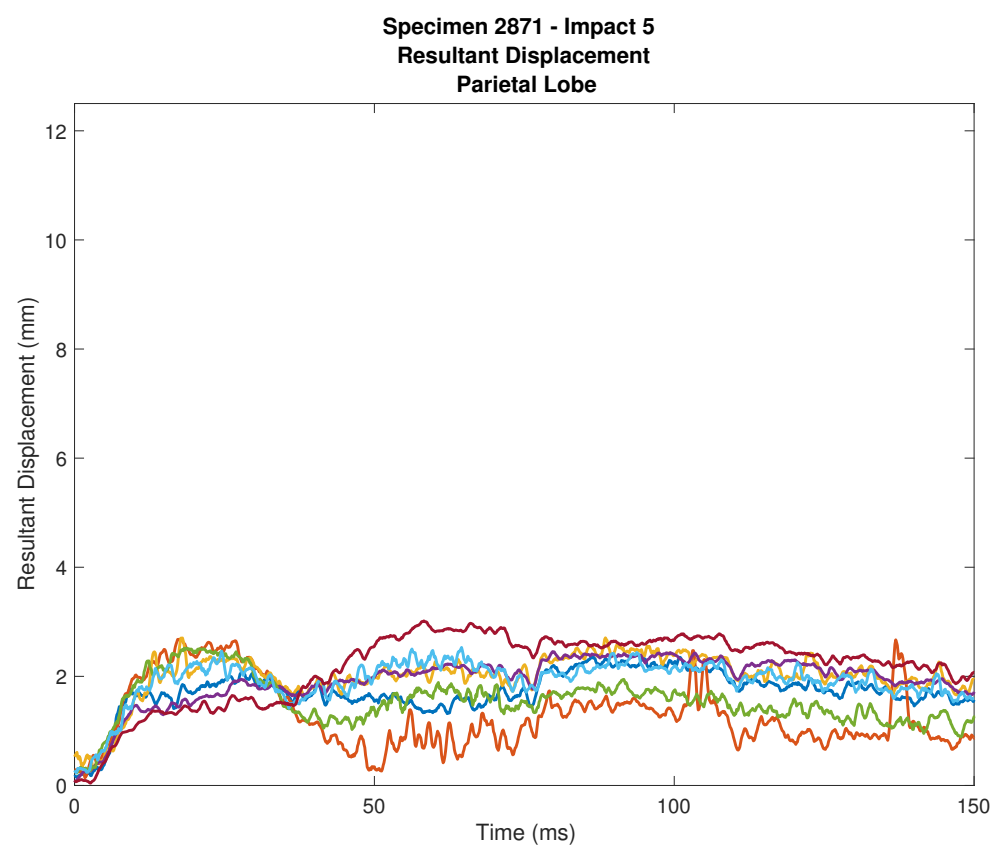

Figure 124: Displacement of parietal lobe - 2871-5 


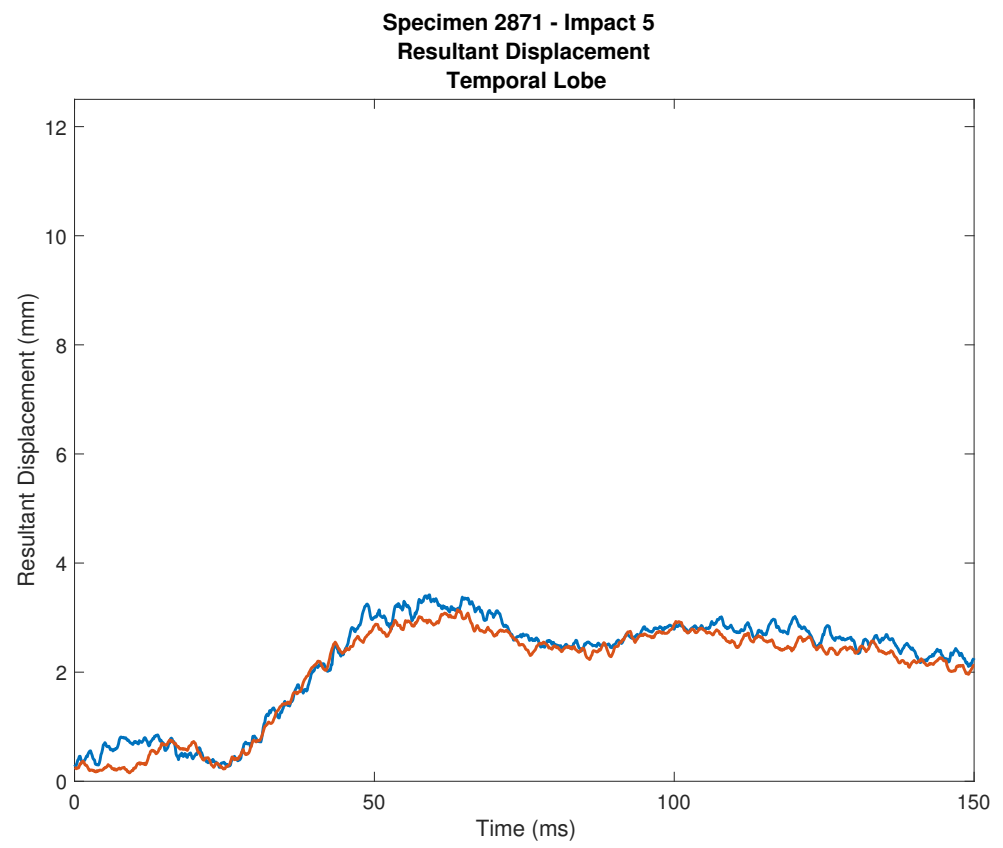

Figure 125: Displacement of temporal lobe - 2871-5

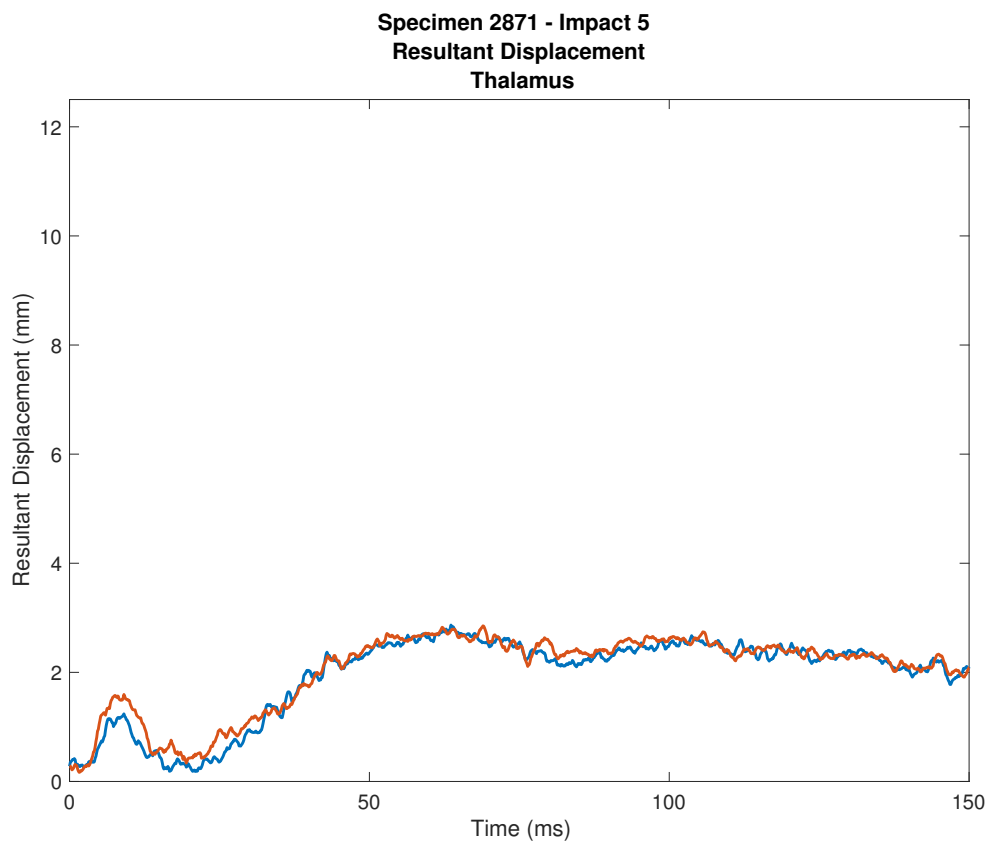

Figure 126: Displacement of thalamus - 2871-5 


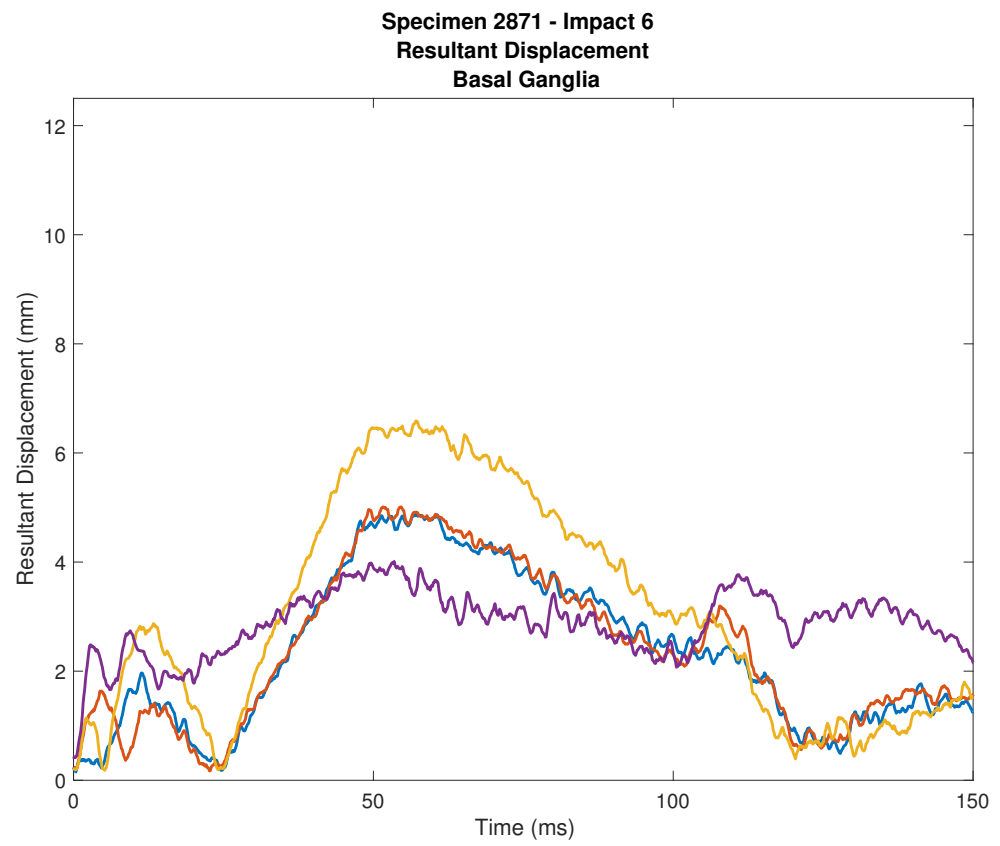

Figure 127: Displacement of basal ganglia - 2871-6

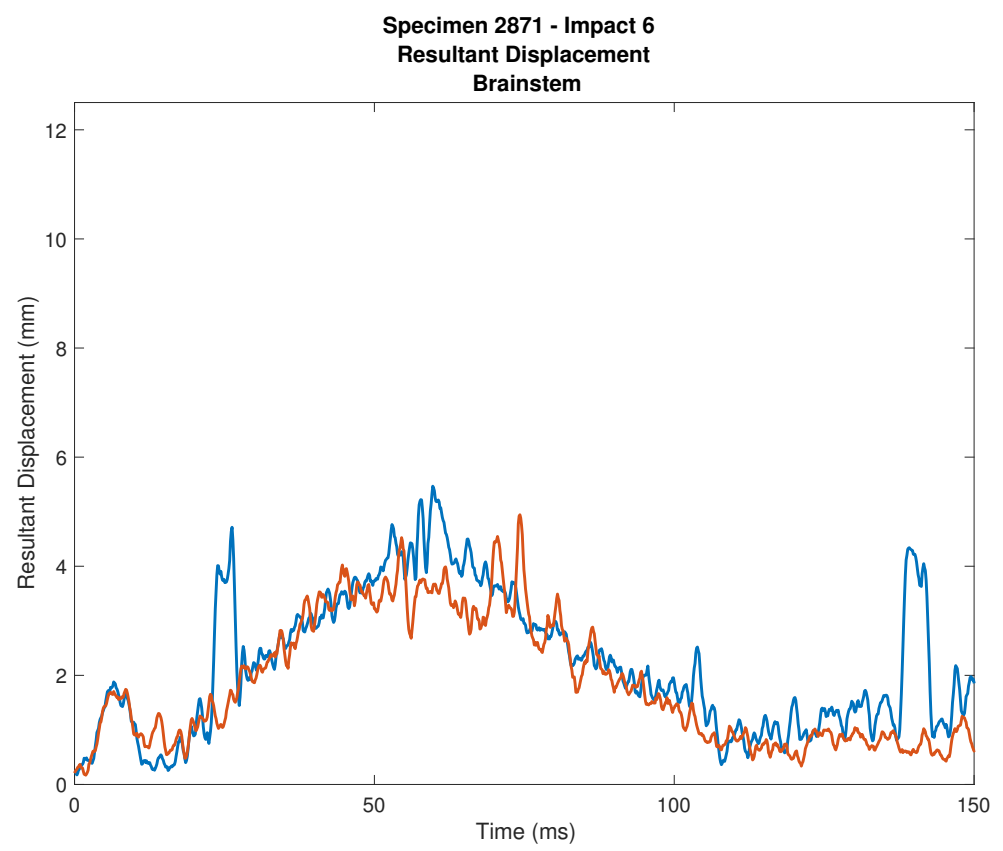

Figure 128: Displacement of brainstem - 2871-6 


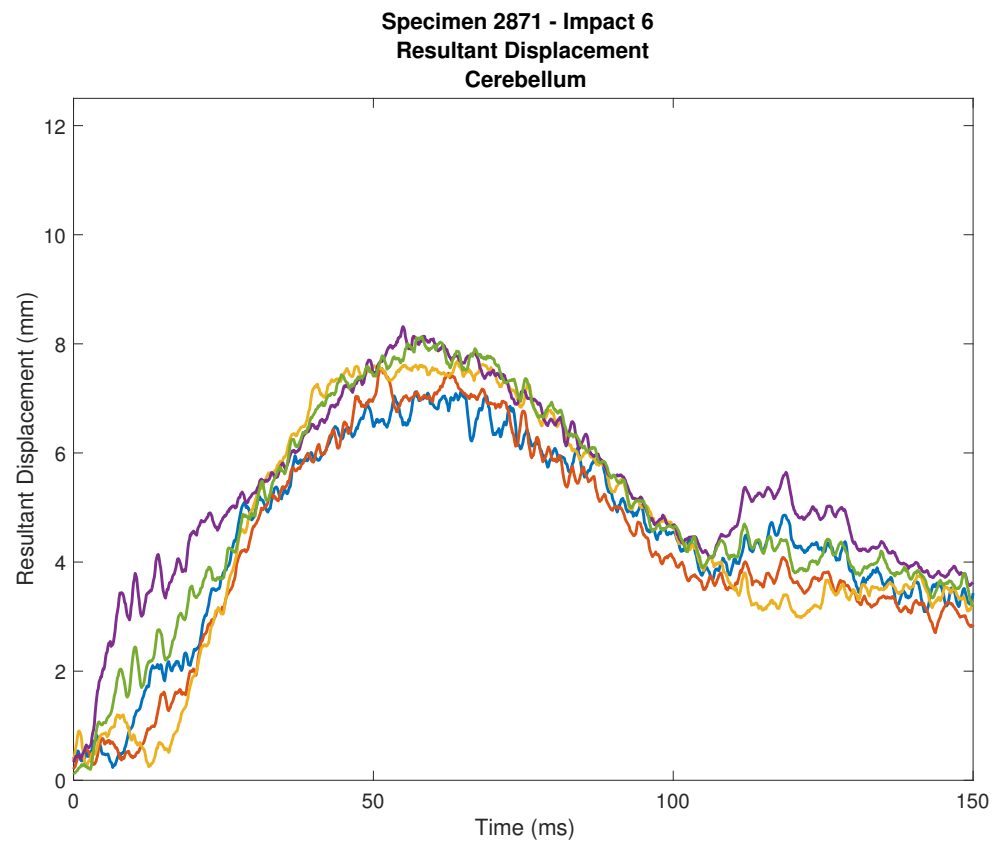

Figure 129: Displacement of cerebellum - 2871-6

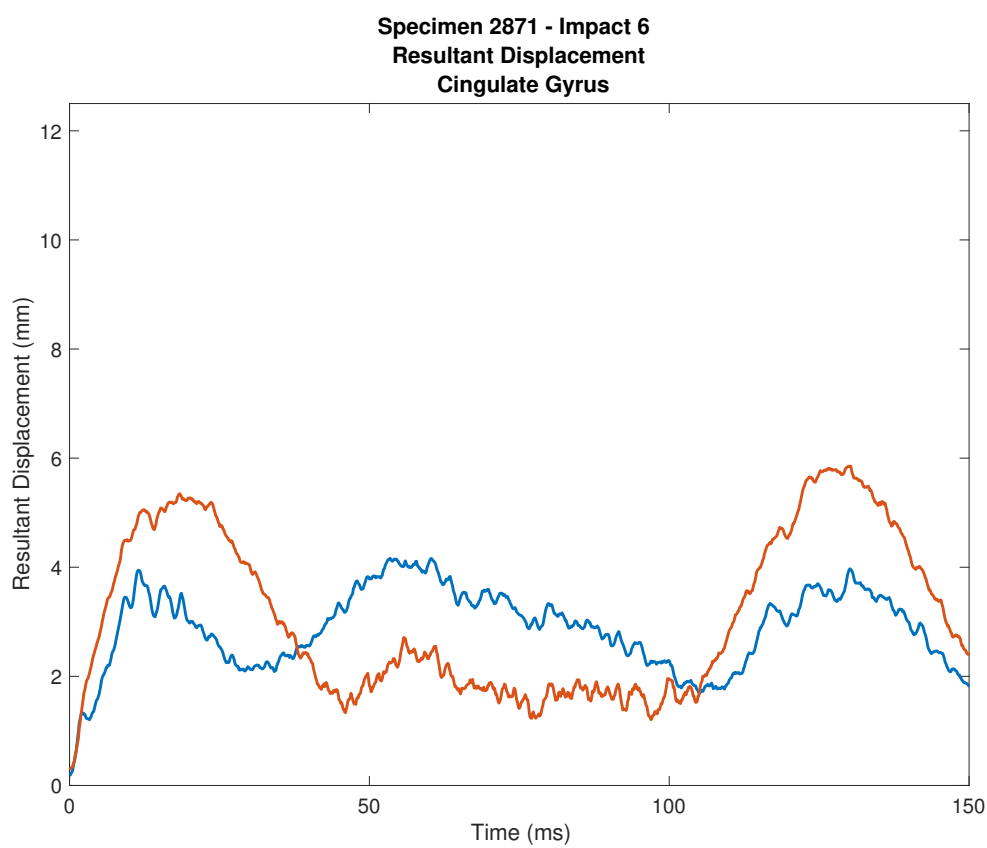

Figure 130: Displacement of cingulate gyrus - 2871-6 


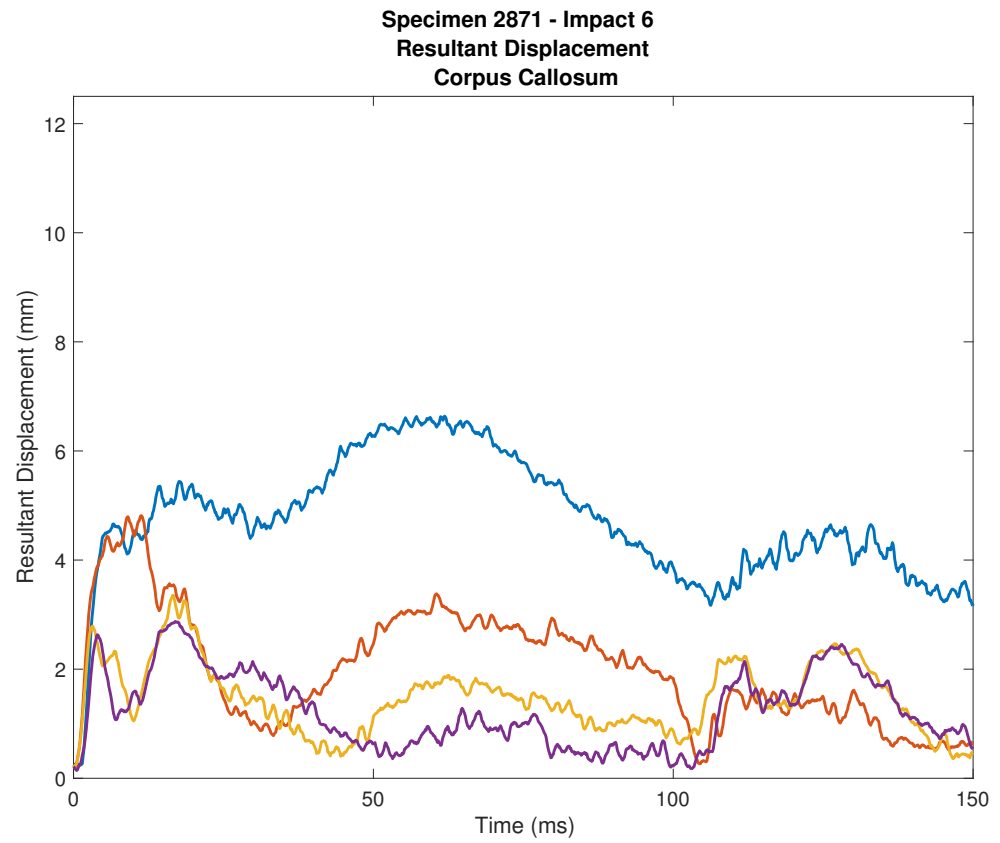

Figure 131: Displacement of corpus callosum - 2871-6

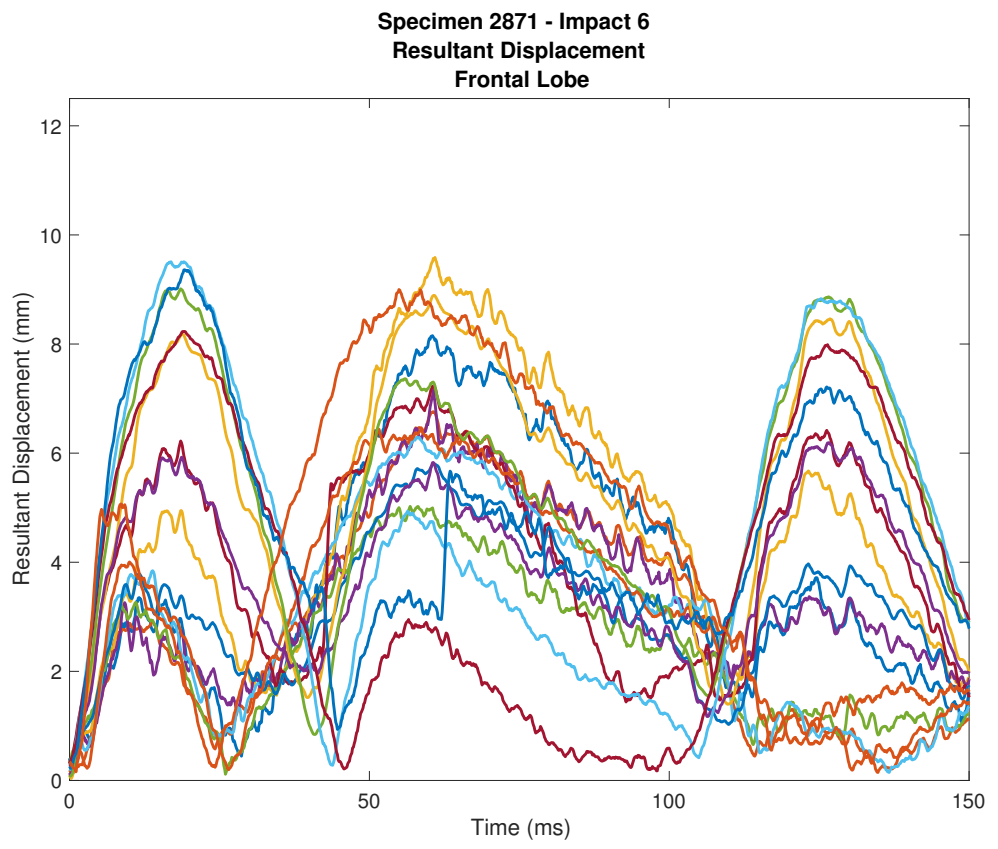

Figure 132: Displacement of frontal lobe - 2871-6 


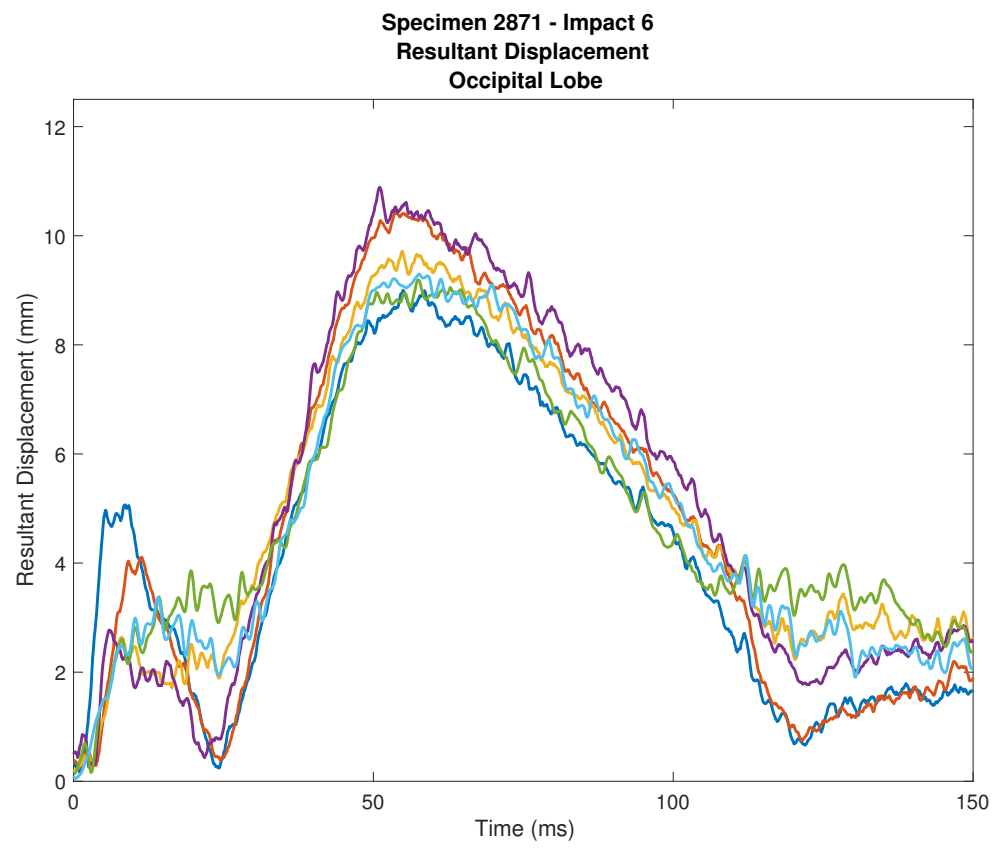

Figure 133: Displacement of occipital lobe - 2871-6

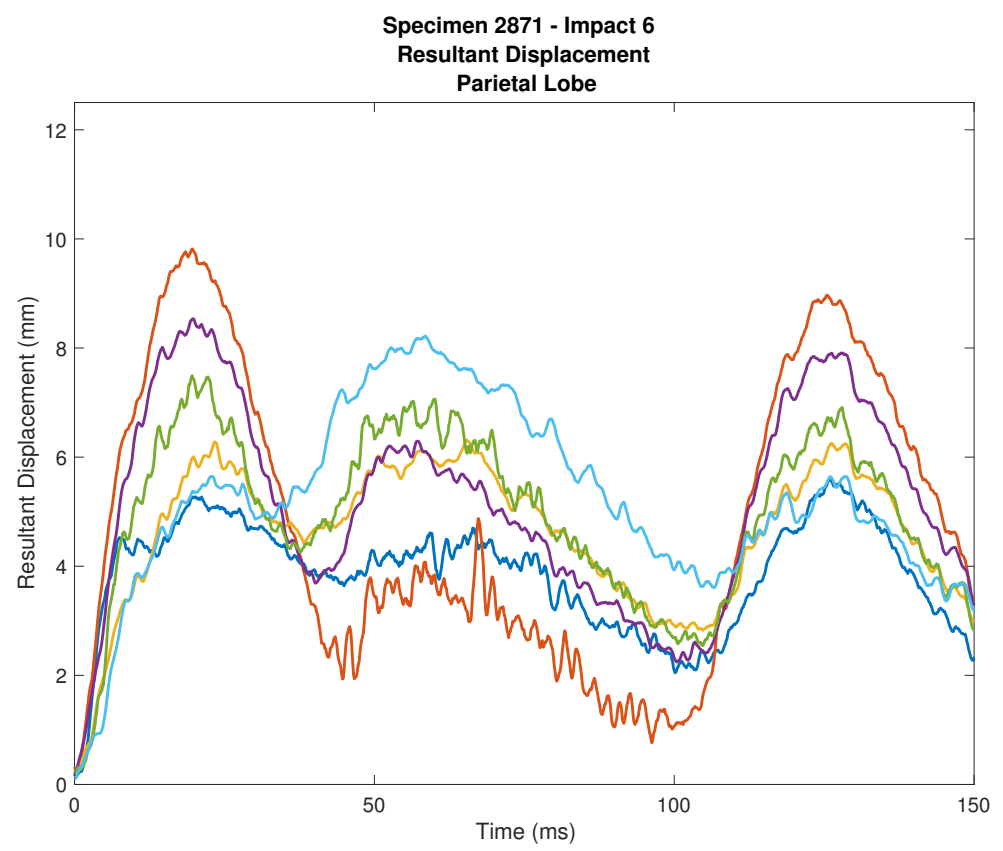

Figure 134: Displacement of parietal lobe - 2871-6 


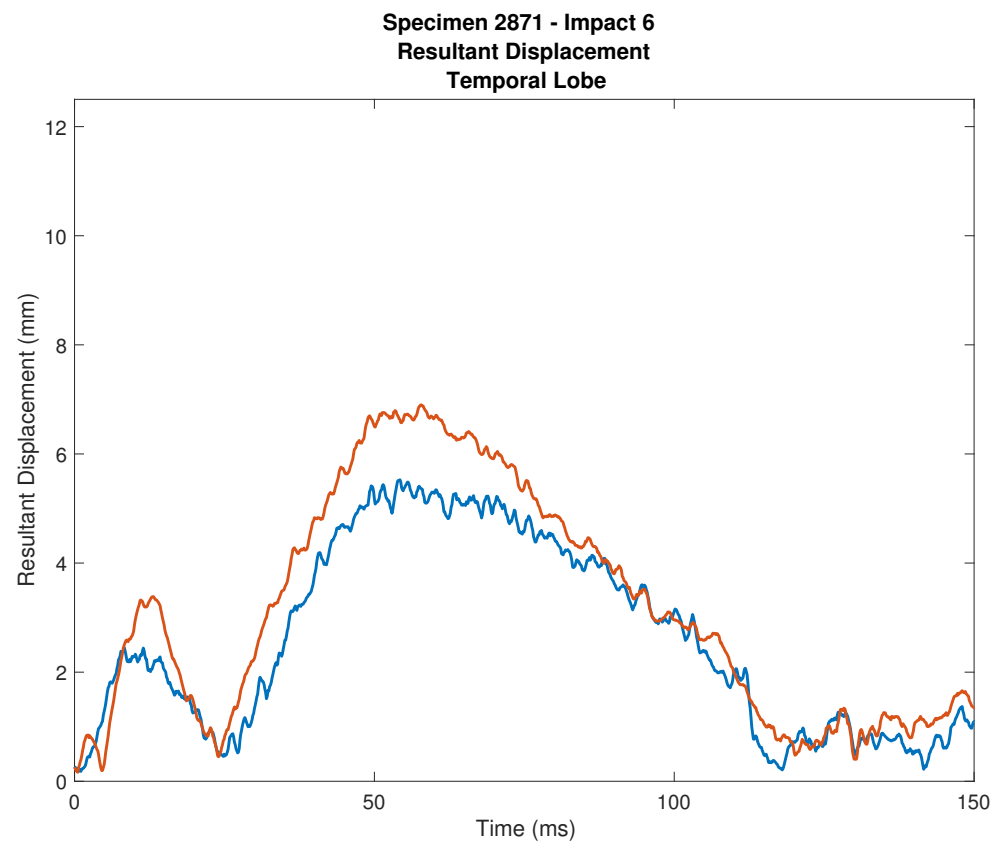

Figure 135: Displacement of temporal lobe - 2871-6

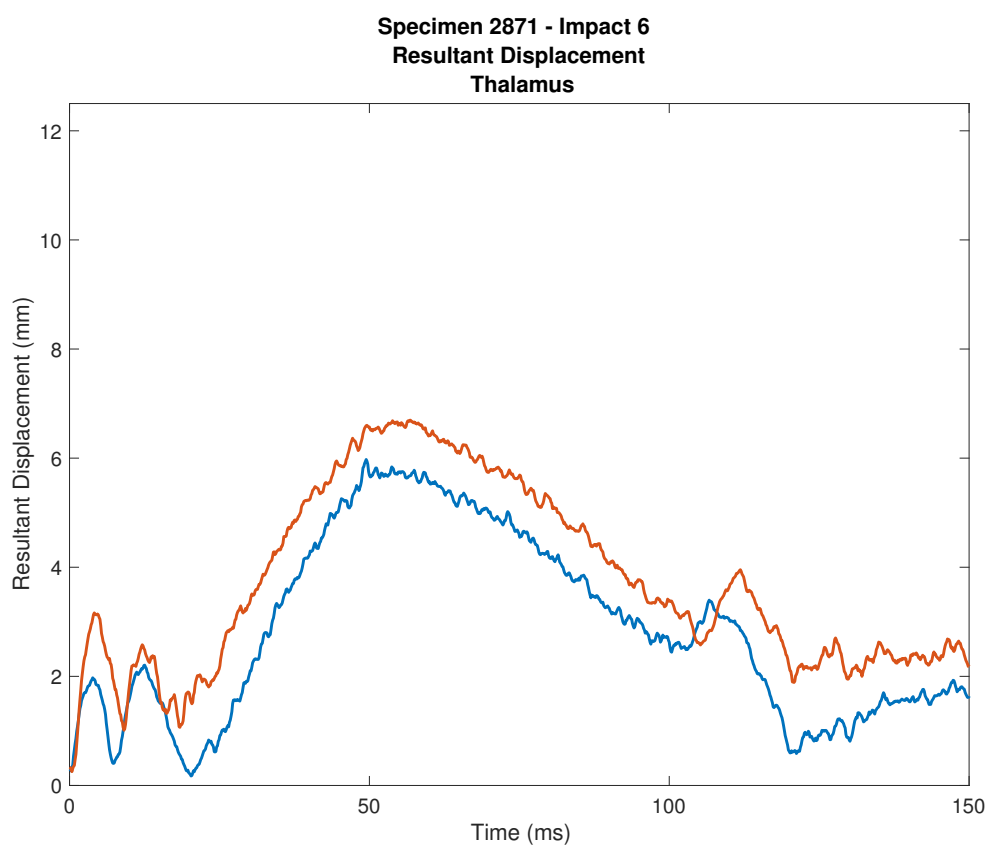

Figure 136: Displacement of thalamus - 2871-6 


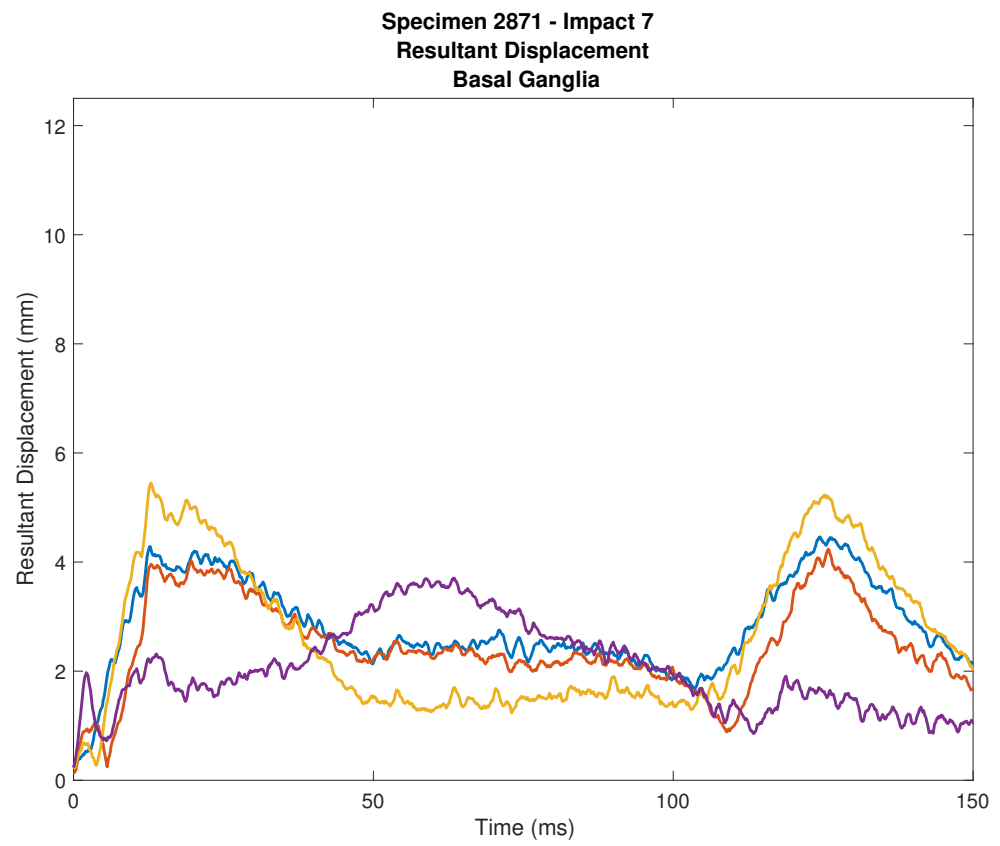

Figure 137: Displacement of basal ganglia - 2871-7

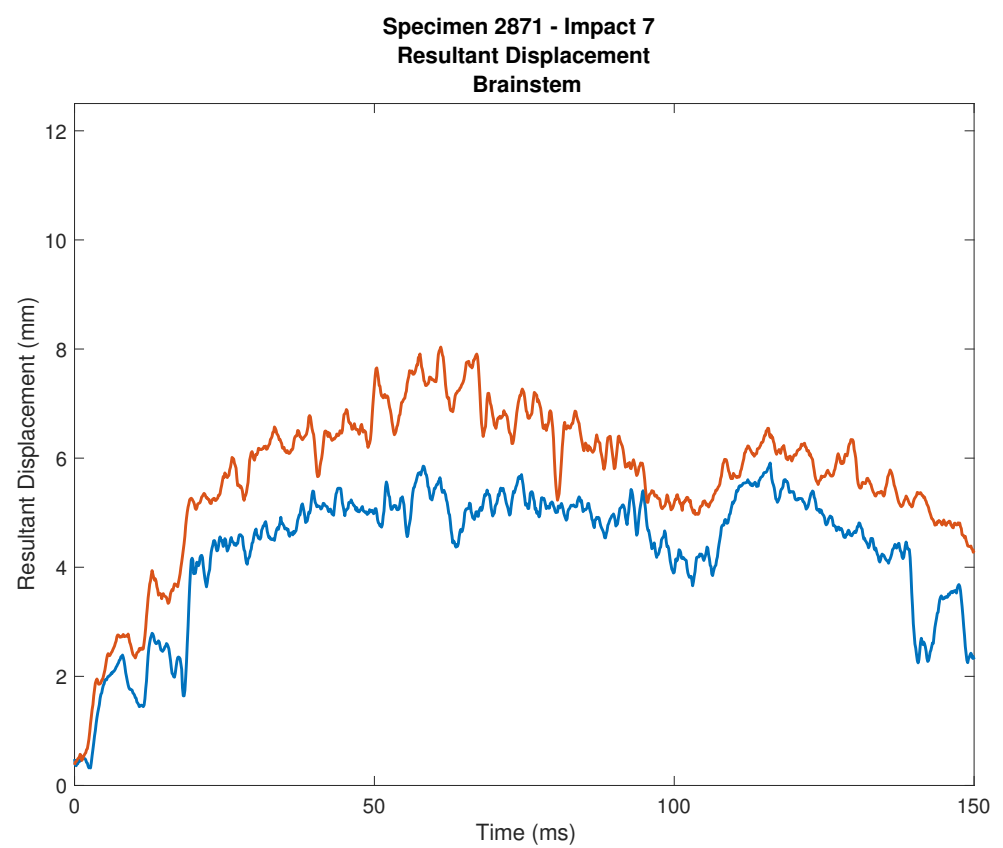

Figure 138: Displacement of brainstem - 2871-7 


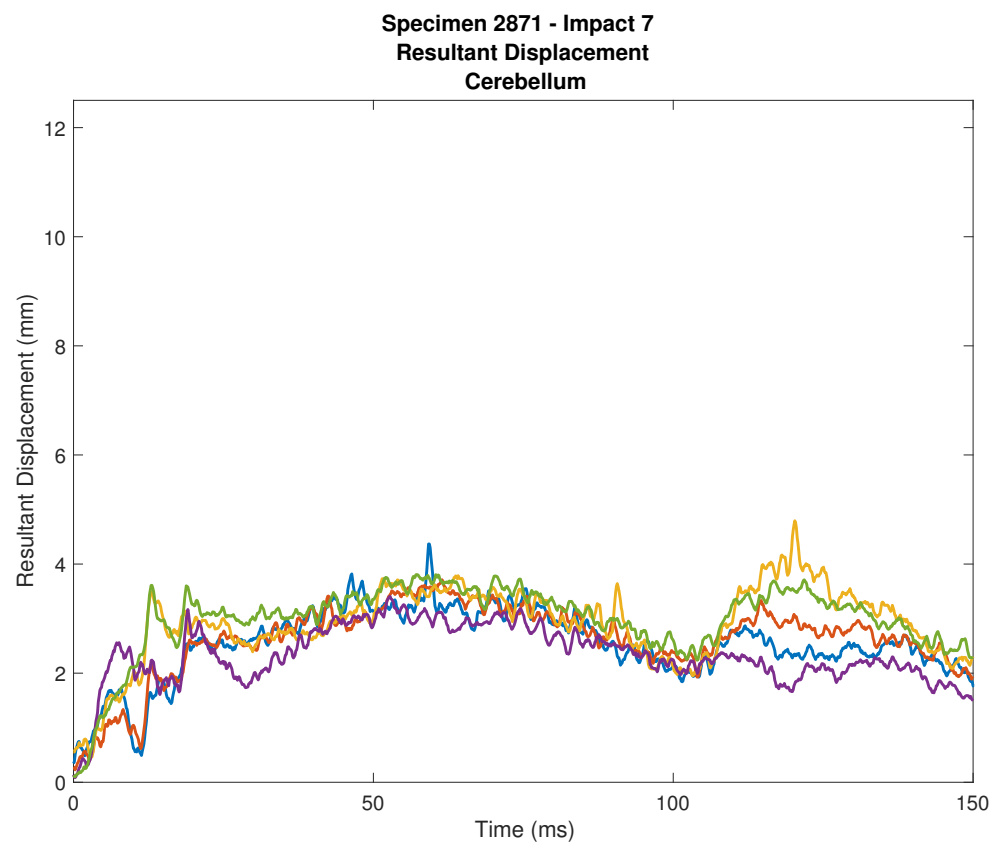

Figure 139: Displacement of cerebellum - 2871-7

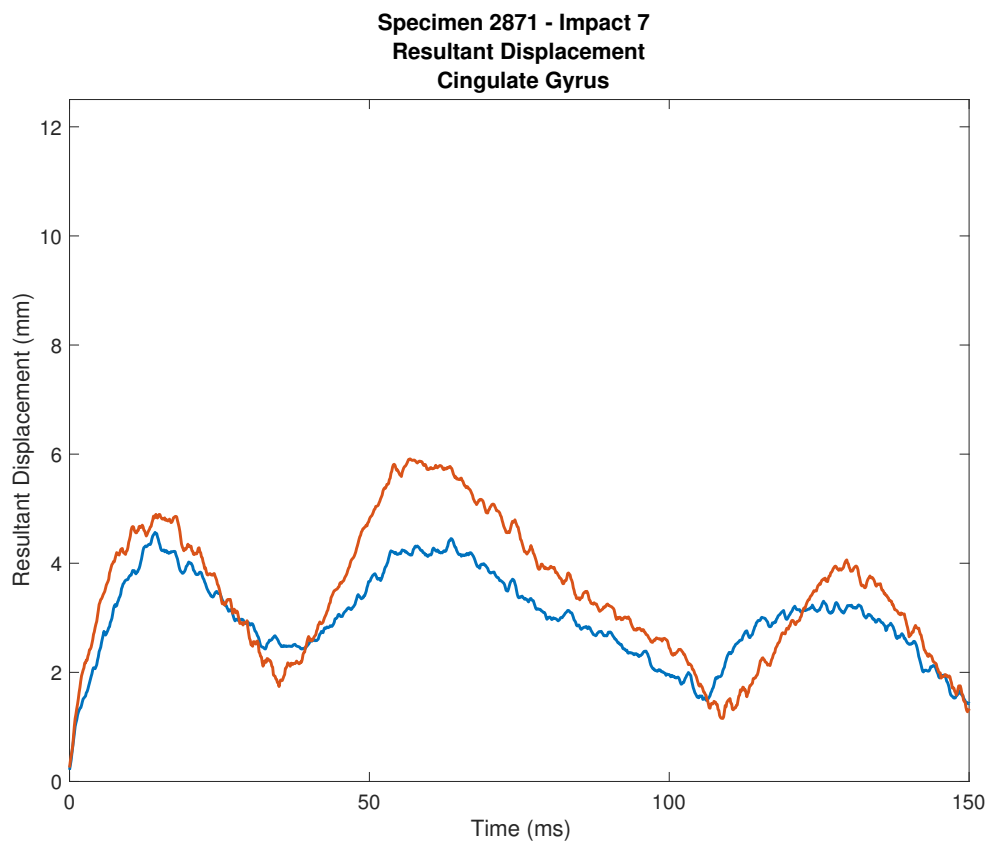

Figure 140: Displacement of cingulate gyrus - 2871-7 


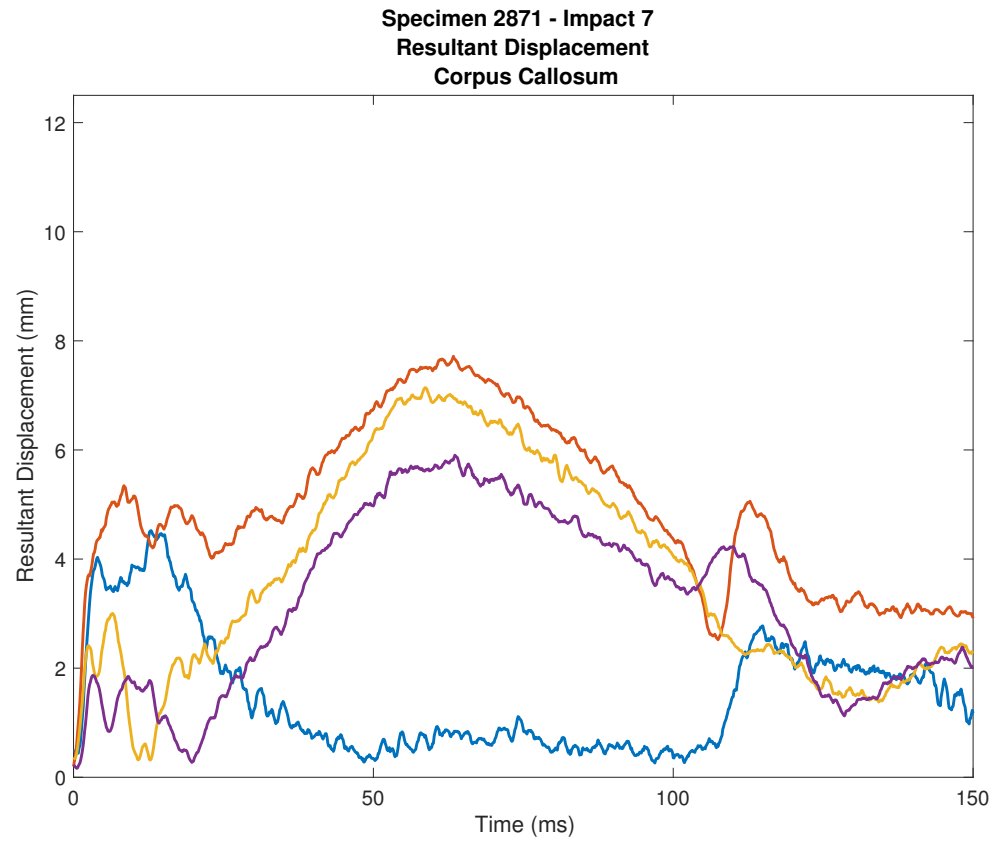

Figure 141: Displacement of corpus callosum - 2871-7

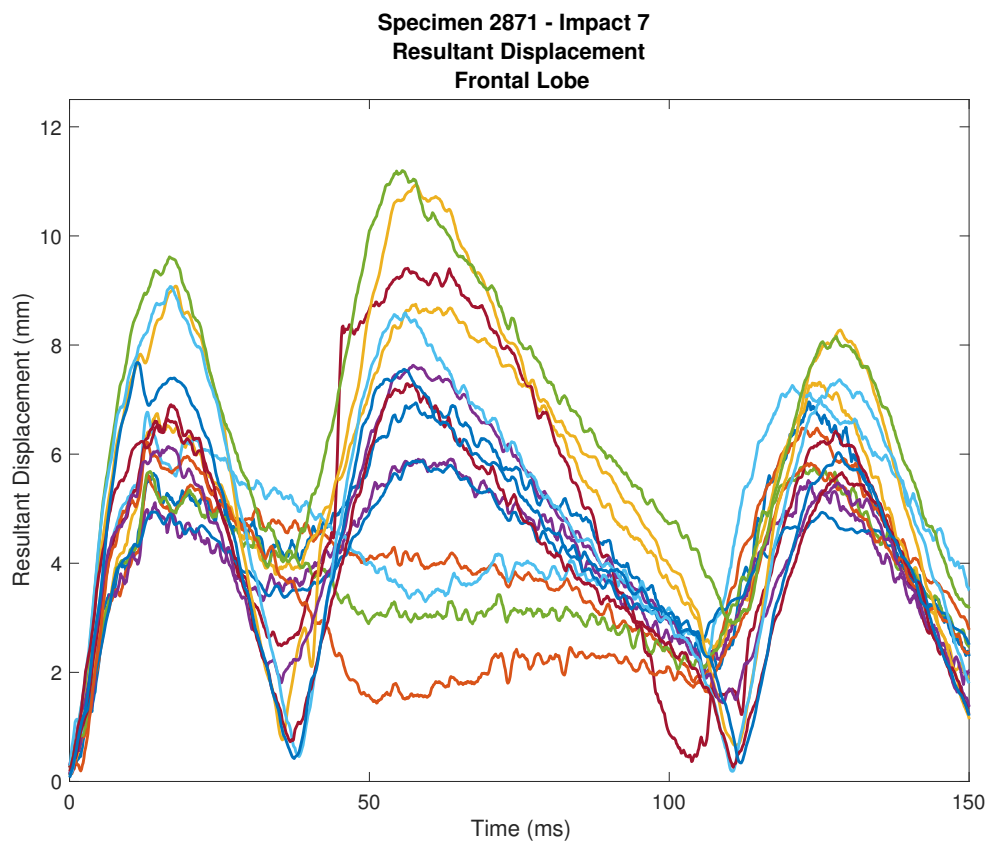

Figure 142: Displacement of frontal lobe - 2871-7 


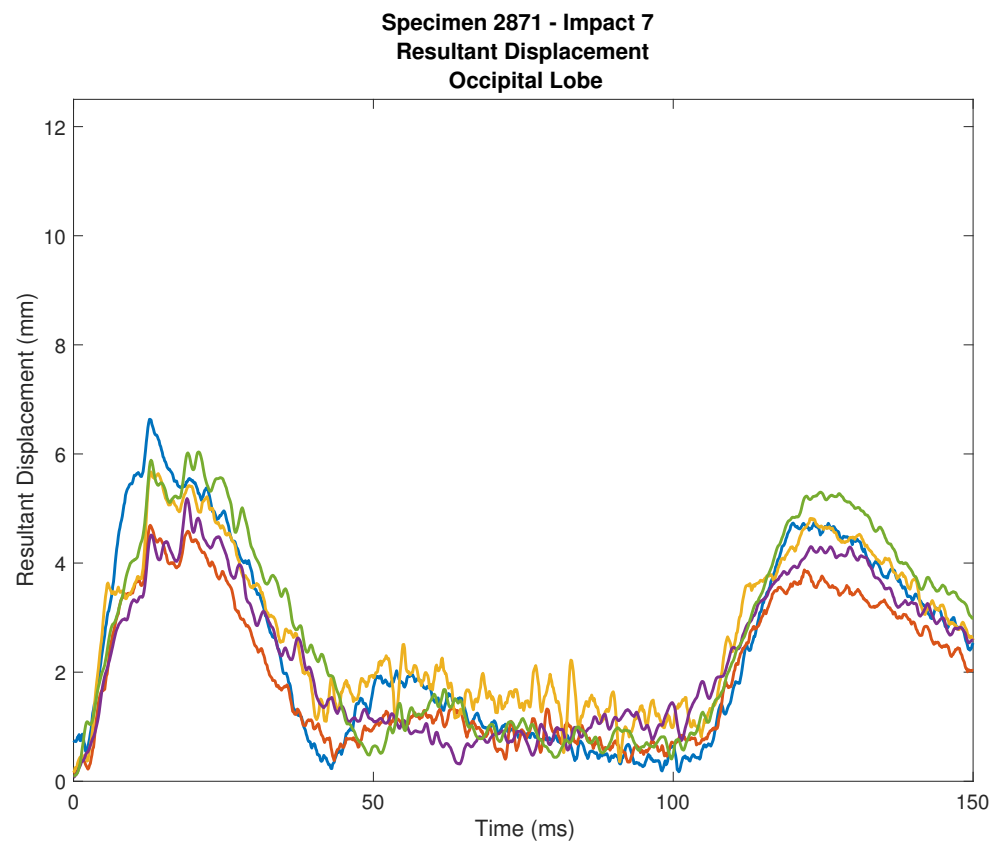

Figure 143: Displacement of occipital lobe - 2871-7

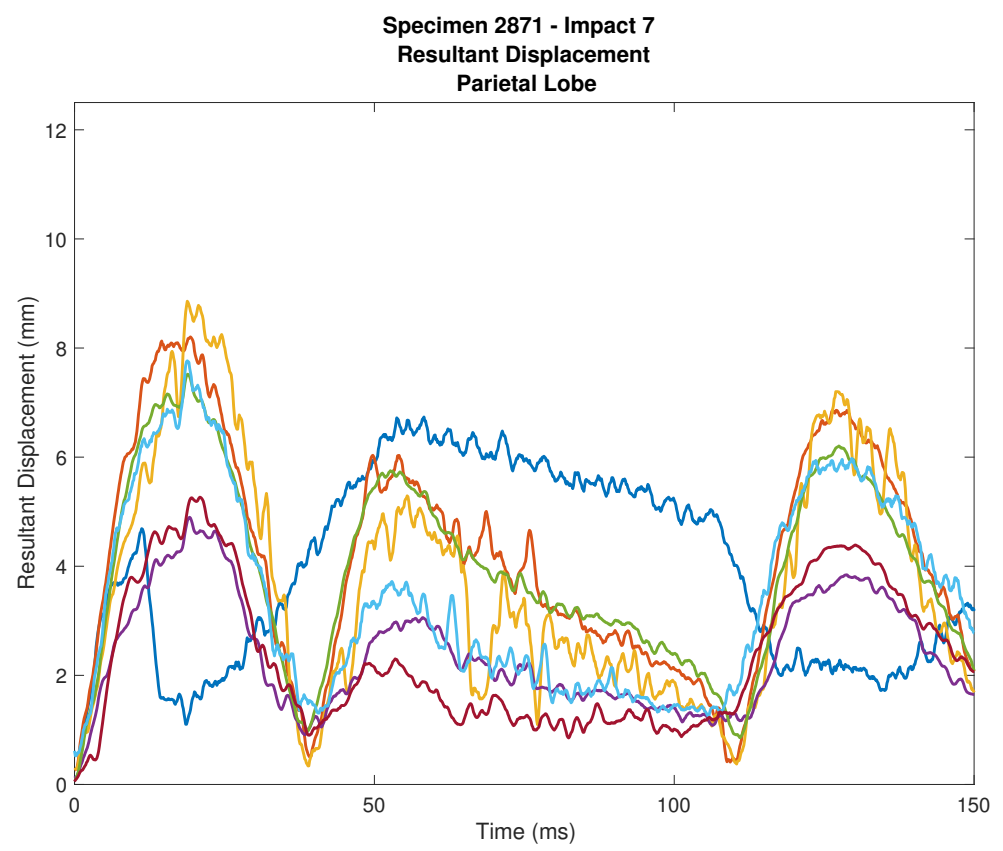

Figure 144: Displacement of parietal lobe - 2871-7 


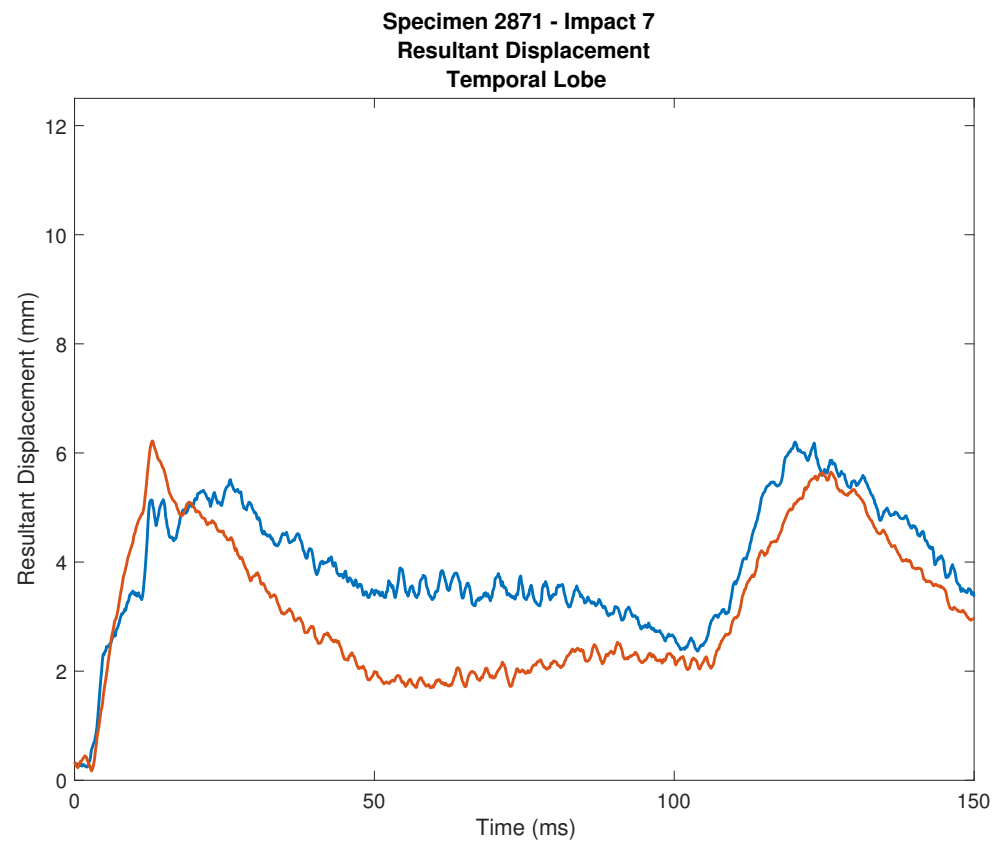

Figure 145: Displacement of temporal lobe - 2871-7

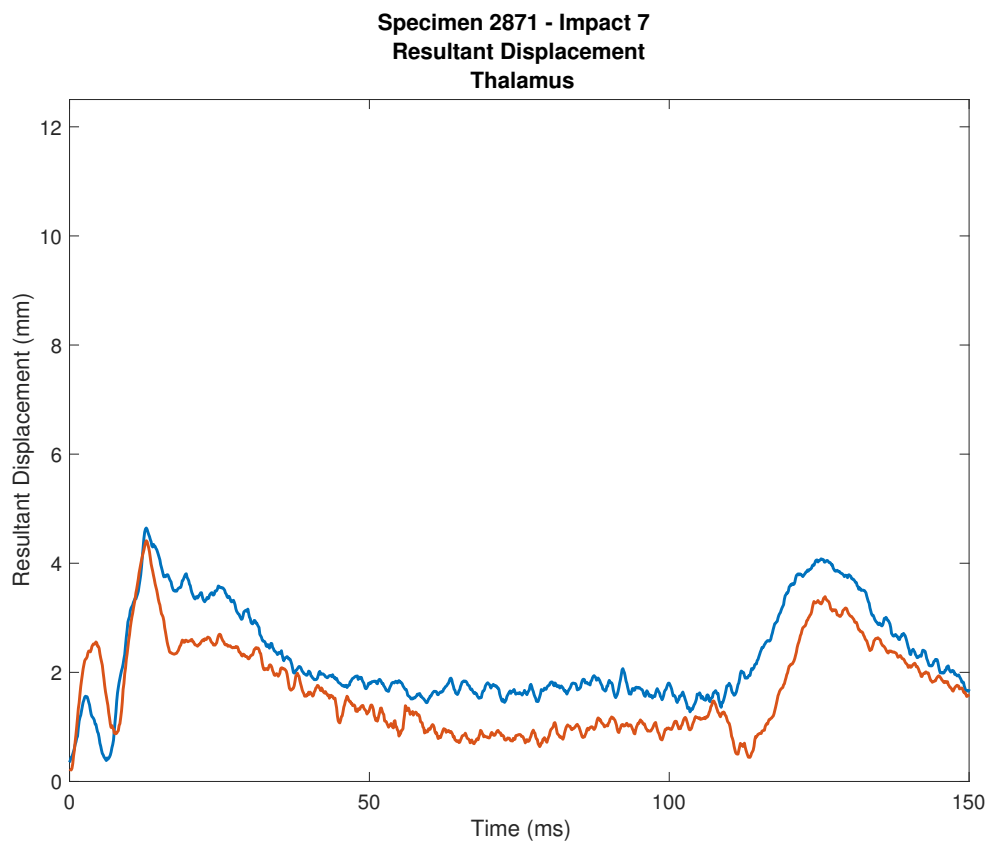

Figure 146: Displacement of thalamus - 2871-7 


\section{Appendix E}

\section{Excluded Markers}

The markers excluded from analysis for Specimen 2842 are listed in Table 17, All

markers exhibiting (A) non conforming motion, or (B) poor tracking due to image clarity or interference were excluded. As MRI confirmation of marker placement was not available for this specimen, once markers were flagged for exclusion they were excluded for all impacts, regardless of better performance in earlier or later impacts.

The markers excluded from analysis for Specimen 2871 are listed in Table 18 Markers determined to be not embedded in tissue via MRI analysis were excluded from all impacts $(\mathrm{C})$. For individual trials, some markers exhibited interference or clarity issues, and were excluded on an impact-by-impact basis (D). 
Table 17: Marker exclusions - Specimen 2842

\begin{tabular}{cc}
\hline Marker & Reason \\
\hline 4 & $\mathrm{~A}$ \\
5 & $\mathrm{~B}$ \\
8 & $\mathrm{~B}$ \\
25 & $\mathrm{~B}$ \\
34 & $\mathrm{~B}$ \\
35 & $\mathrm{~A}$ \\
39 & $\mathrm{~B}$ \\
44 & $\mathrm{~A}$ \\
47 & $\mathrm{~A}$ \\
48 & $\mathrm{~A}$ \\
52 & $\mathrm{~A}$ \\
56 & $\mathrm{~A}$ \\
61 & $\mathrm{~B}$ \\
64 & $\mathrm{~B}$ \\
65 & $\mathrm{~B}$ \\
67 & $\mathrm{~B}$ \\
69 & $\mathrm{~B}$ \\
70 & $\mathrm{~B}$ \\
71 & $\mathrm{~B}$ \\
72 & $\mathrm{~B}$ \\
74 & $\mathrm{~B}$ \\
75 & $\mathrm{~B}$ \\
\hline
\end{tabular}

Table 18: Marker exclusions - Specimen 2871

\begin{tabular}{c|cc}
\hline Impact & Markers & Reason \\
\hline All & $26,27,36,42,45$ & C \\
\hline 1 & 54 & D \\
3 & 12,53 & D \\
4 & $6,37,53$ & D \\
5 & 52 & D \\
6 & 40 & D \\
7 & 34,54 & D \\
\hline
\end{tabular}




\section{Appendix F}

\section{Additional Imaging and MRI}

This appendix contains MRI images showing the position of embedded contrast markers. All markers embedded in Specimen 2842 are shown in Figure 147 and were inserted in a single plane. Specimen 2871 was embedded in three different planes, including the primary parasagittal plane (Figure 148), the corpus callosum (Figure 149), and the brain stem (Figure 150). Anterior (A) and posterior (P) are indicated in each figure. Marker positions as determined by neurosurgeon partners are listed in Table 19 .

Table 19: Location of markers per MRI review - Specimen 2871

\begin{tabular}{cc}
\hline Marker & Location \\
1 & rostral prefrontal cortex \\
2 & orbital frontal cortex \\
3 & rostral prefrontal cortex \\
4 & prefrontal subcortical cortex \\
5 & prefrontal subcortical cortex \\
\hline & Continued on next page
\end{tabular}


Table 19 - continued from previous page

\begin{tabular}{cc}
\hline Marker & Location \\
\hline 6 & frontal gyrus rectus cortex \\
7 & prefrontal subcortical cortex \\
8 & prefrontal cortex \\
9 & caudate nucleus : Basal ganglia \\
10 & ventromedial prefrontal cortex \\
11 & mesial temporal cortex \\
12 & superior frontal gyrus cortex \\
13 & superior frontal gyrus cortex \\
14 & cingulate gyrus cortex \\
15 & putamen: basal ganglia \\
16 & globus pallidus: basal ganglia \\
17 & superior frontal gyrus cortex \\
18 & cingulate gyrus cortex \\
19 & caudate nucleus : Basal ganglia \\
20 & thalamus \\
21 & parahippocampal gyrus ( posterior medial temporal cortex) \\
22 & superior frontal gyrus cortex \\
23 & superior frontal gyrus cortex \\
24 & frontal subcortical cortex \\
25 & thalamus \\
26 & parahippocampal gyrus ( posterior medial temporal cortex) \\
27 & dentate nucleus of cerebellum \\
28 & \\
\hline & \\
\hline &
\end{tabular}


Table 19 - continued from previous page

\begin{tabular}{|c|c|}
\hline Marker & Location \\
\hline 29 & deep nuclei of the cerebellum \\
\hline 30 & cerebellar hemisphere \\
\hline 31 & frontal cortex \\
\hline 32 & parietal subcortical cortex \\
\hline 33 & splenium of the corpus callosum \\
\hline 34 & lingual gyrus occiptal lobe \\
\hline 35 & lingual gyrus occiptal lobe \\
\hline 36 & subarachnoid space/ not in brain tissue \\
\hline 37 & cerebellar hemisphere \\
\hline 38 & cerebellar hemisphere \\
\hline 39 & parietal cortex \\
\hline 40 & parietal cortex \\
\hline 41 & precuneus parietal cortex \\
\hline 42 & in the parietooccipital sulcus /not in brain \\
\hline 43 & occipital lobe near calcarine fissure \\
\hline 44 & inferior occipital lobe \\
\hline 45 & subarachoid space \\
\hline 46 & precuneus parietal cortex. \\
\hline 47 & precuneus parietal cortex. \\
\hline 48 & precuneus parietal cortex \\
\hline 49 & Body of corpus callosum \\
\hline 50 & Body of corpus callosum \\
\hline \multirow[t]{2}{*}{51} & splenium of the corpus callosum \\
\hline & Continued on $\mathrm{n}$ \\
\hline
\end{tabular}


Table 19 - continued from previous page

\begin{tabular}{cc}
\hline Marker & Location \\
\hline 52 & pons: brainstem \\
53 & medulla : brainstem \\
54 & in the subarachnoid space at cervicalmedullary junction \\
55 & superior occipital lobe \\
56 & superior occipital lobe \\
\hline \hline
\end{tabular}




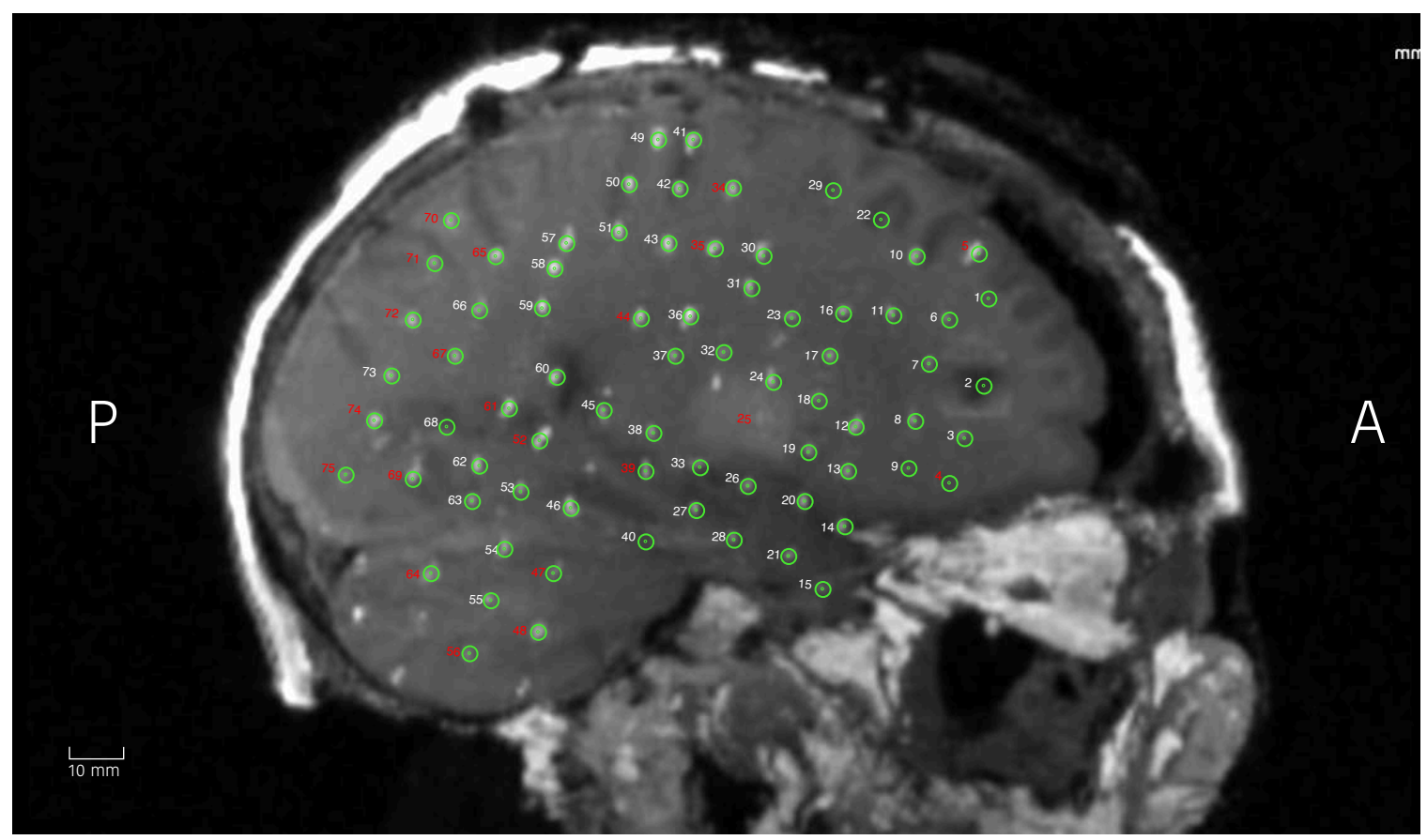

Figure 147: MRI image of parasagittal slice, approximately $5 \mathrm{~mm}$ thick with center at $17 \mathrm{~mm}$ from mid-sagittal. Markers highlighted with numbers used for tracking.

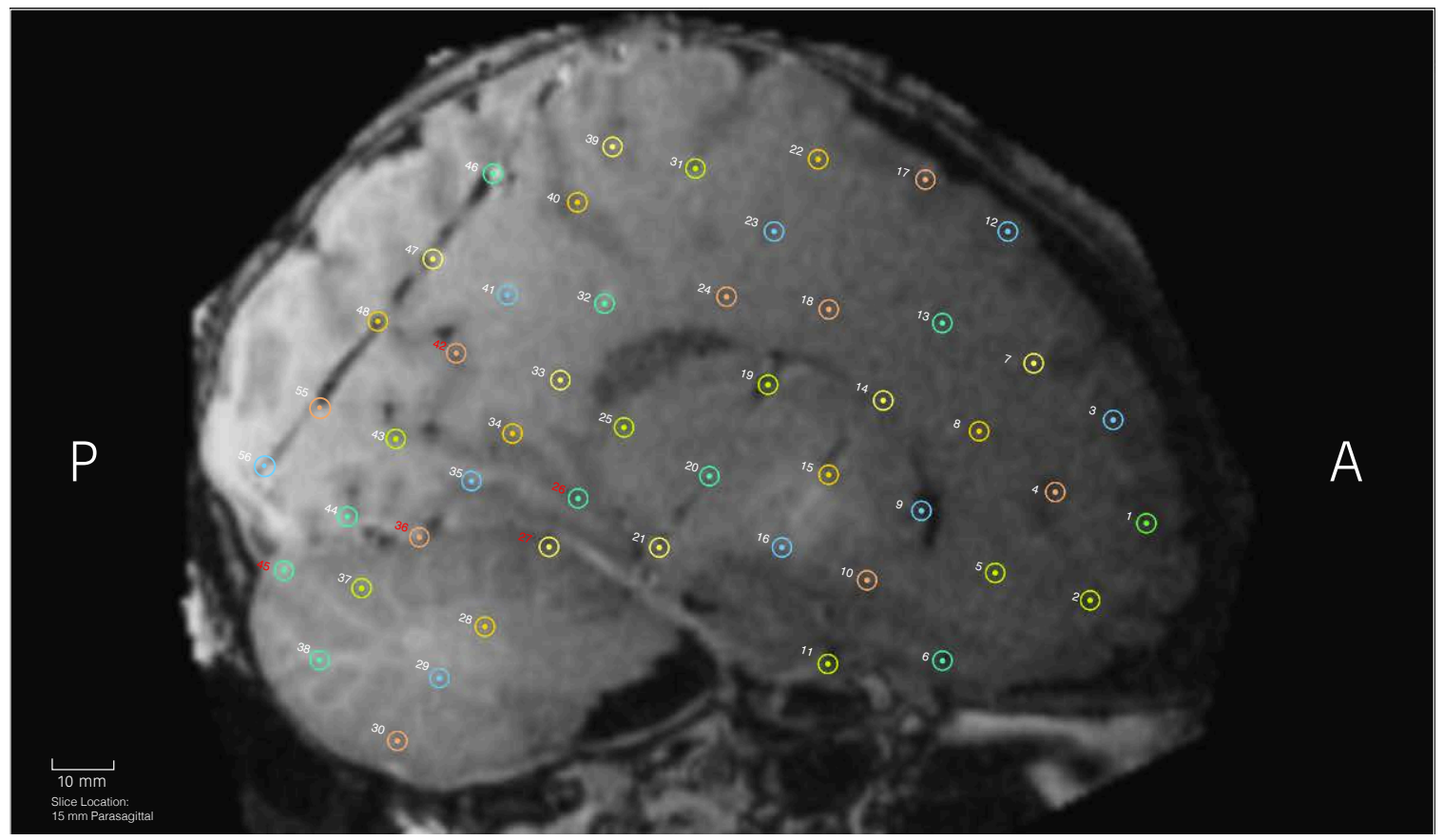

Figure 148: MRI image of parasagittal slice, approximately $3 \mathrm{~mm}$ thick. Markers highlighted with numbers used for tracking. 


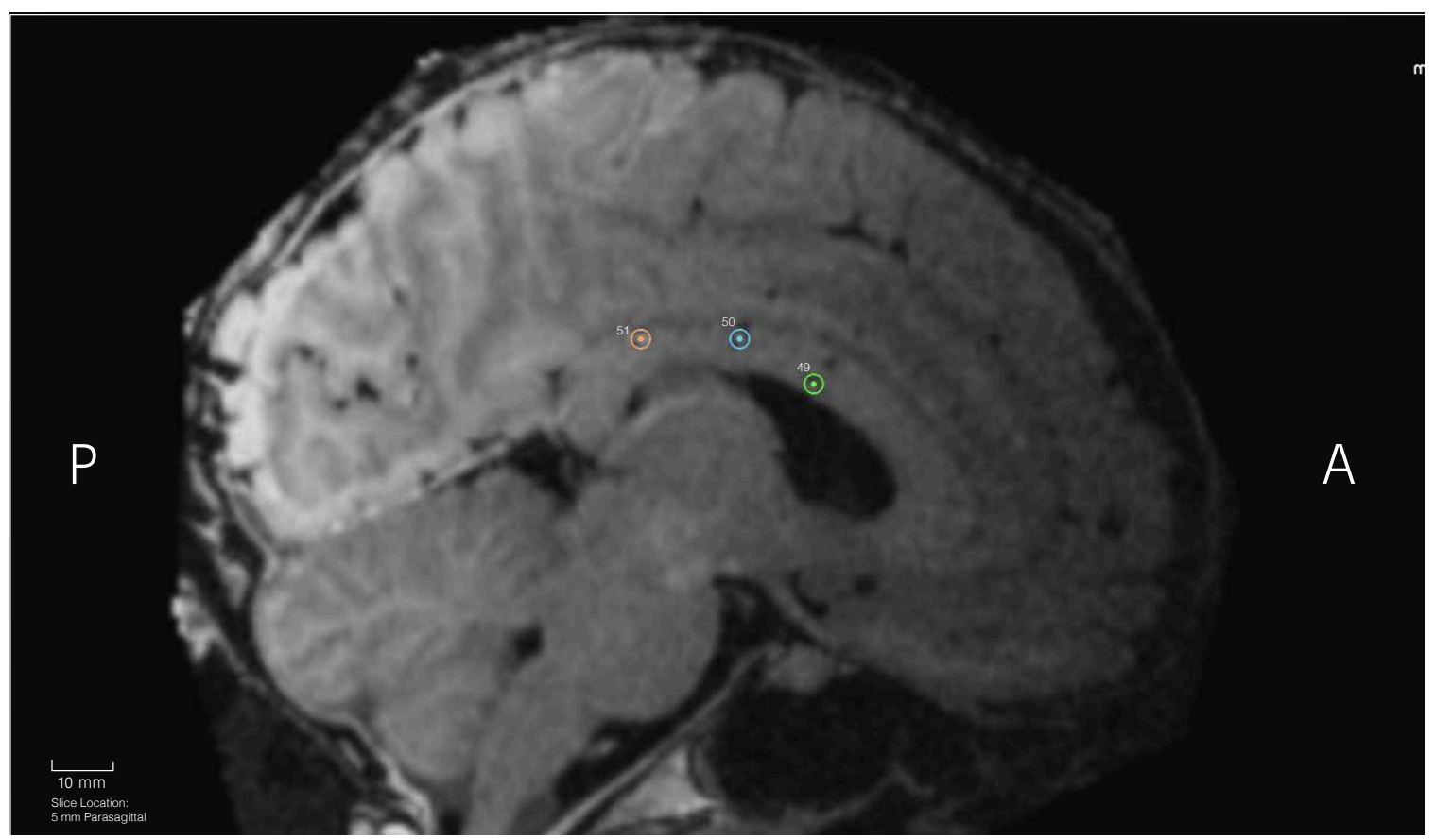

Figure 149: MRI image of parasagittal slice, approximately $2 \mathrm{~mm}$ thick, showing markers embedded in corpus callosum. Markers highlighted with numbers used for tracking.

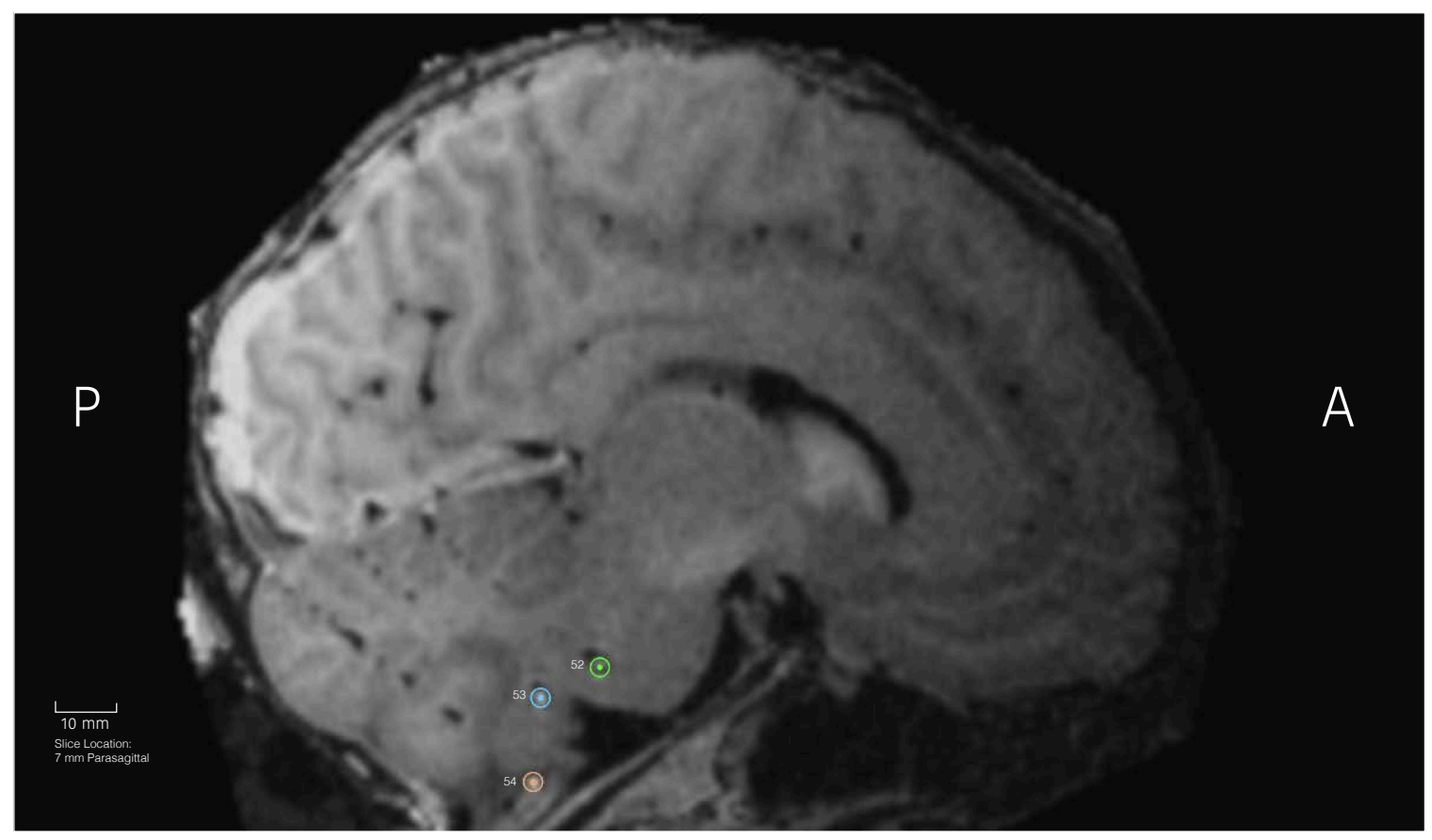

Figure 150: MRI image of parasagittal slice, approximately $2 \mathrm{~mm}$ thick, showing markers embedded in brain stem. Markers highlighted with numbers used for tracking. 